\title{
Modelling on the nanomechanics of cytoskeletal filaments
}

\section{$\mathrm{Li}, \mathrm{Si}$}

How to cite:

$\mathrm{Li}, \mathrm{Si}$ (2019) Modelling on the nanomechanics of cytoskeletal filaments. Doctoral thesis, Swansea University. http://cronfa.swan.ac.uk/Record/cronfa52432

Use policy:

This item is brought to you by Swansea University. Any person downloading material is agreeing to abide by the terms of the repository licence: copies of full text items may be used or reproduced in any format or medium, without prior permission for personal research or study, educational or non-commercial purposes only. The copyright for any work remains with the original author unless otherwise specified. The full-text must not be sold in any format or medium without the formal permission of the copyright holder. Permission for multiple reproductions should be obtained from the original author.

Authors are personally responsible for adhering to copyright and publisher restrictions when uploading content to the repository.

Please link to the metadata record in the Swansea University repository, Cronfa (link given in the citation reference above.)

http://www.swansea.ac.uk/library/researchsupport/ris-support/ 


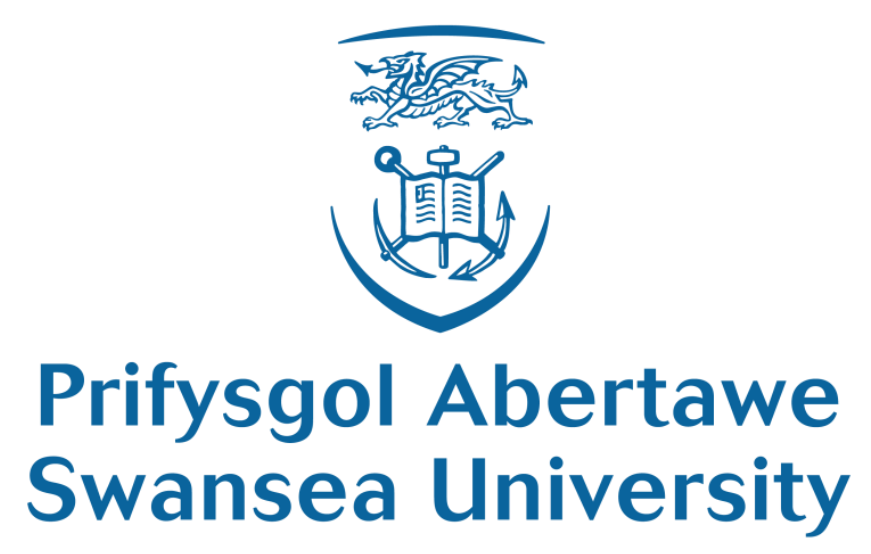

\section{Modelling on the nanomechanics of cytoskeletal filaments}

$\mathrm{Si} \mathrm{Li}$

Swansea University

June, 2019 


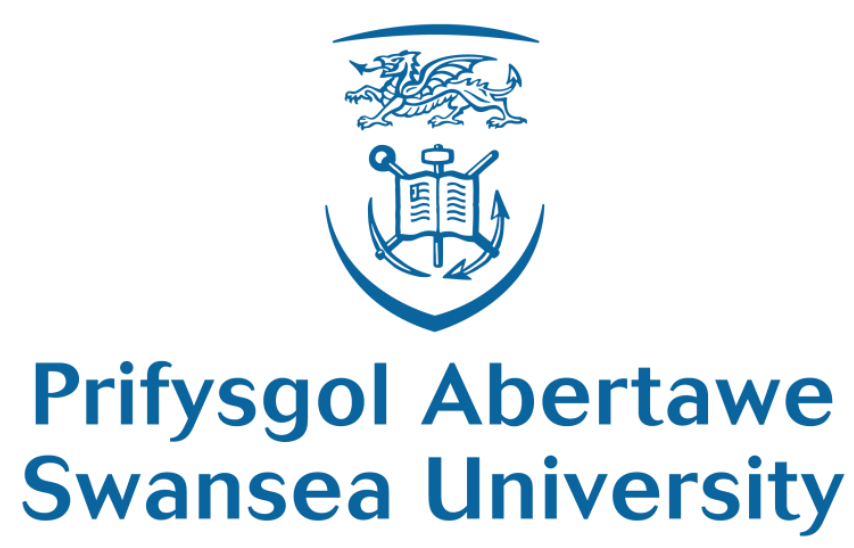

\title{
Modelling on the nanomechanics of cytoskeletal filaments
}

\author{
by $\mathrm{Si} \mathrm{Li}$
}

Supervised by Dr. Chengyuan Wang

Professor Perumal Nithiarasu

Submitted to Swansea University in fulfilment of the requirements for the Degree of

Doctor of Philosophy in

Mechanical Engineering

Swansea University

June, 2019 


\begin{abstract}
Cytoskeleton is a structure that enables cells to maintain their shape and internal organization. The proper functions of cytoskeleton depend crucially on the mechanical responses and properties of its component filaments (e.g., microtubules and actin filaments). Thus, an in-depth understanding of cytoskeletal filaments mechanics is essential in revealing how cells fulfil their biological functions via cytoskeleton, stimulating the innovative idea in designing biomimetic structure or materials and facilitating to develop novel techniques in disease diagnosis and treatment. This thesis thus focuses on studying the inherent and environmental factors that determine the nanomechanics of cytoskeletal components, i.e., the monomeric feature of cytoskeletal filaments at microscale, the relation between the helical structure and the mechanical properties, and the interaction between the protein filaments and the surrounding environment, such as cytosol, filamentous proteins, electrical fields, etc.

The thesis starts with a comprehensive review of the existing cytoskeletal filaments models. It is followed by the molecular structural mechanics models developed for microtubules and actin filaments. Subsequently, the models with monomeric feature were employed to identify the origin of the inter-protofilament sliding and its role in bending and vibration of microtubules.

After that, helix structure effects on the mechanics of cytoskeletal filaments were explored. A three-dimensional transverse vibration was reported for microtubules with chiral structures, where the bending axis of the cross-section rotates in an anticlockwise direction and the adjacent half-waves oscillate in different planes. The tension-induced bending was also studied for actin filaments as a result of the helicity.

Then, the subcellular environment effect on the filament mechanics was explored. Attempts were also made to reveal the physics of the experimentally observed localized buckling of microtubules and the crucial role of the cross-linker in regulating microtubule stiffness. Also the role of actin-binding proteins in determining the stiffness of actin bundle was examined during the formation of filopodia protrusion.

Finally, the studies were carried out for the microtubule vibration excited by the alternating external electric field. Strong correlation was achieved between the tubulin interaction and the frequency shift. Meanwhile, the unique feature of nanoscale microtubule-cytosol interface was studied in detail. Large reduction of the viscous damping of cytosol was achieved in the presence of the nanoscale solid-liquid interface.

At the end of the thesis, the contributions of the dissertation research were summarised and remarks were given on future research directions.

Keywords: cytoskeleton, nano-filament, microtubule, actin filament, monomeric feature, helical structure, electromechanical vibration
\end{abstract}




\section{DECLARATION}

This work has not previously been accepted in substance for any degree and is not being concurrently submitted in candidature for any degree.

Signed (candidate)

Date

\section{STATEMENT 1}

This thesis is the result of my own investigations, except where otherwise stated. Other sources are acknowledged by footnotes giving explicit references. A bibliography is appended.

Signed (candidate)

Date

\section{STATEMENT 2}

I hereby give consent for my thesis, if accepted, to be available for photocopying and for inter-library loan, and for the title and summary to be made available to outside organisations.

Signed (candidate)

Date 


\section{Acknowledgements}

When I summarised my research works during my $\mathrm{PhD}$ study and completed this thesis, I was so excited that I had made valuable contributions in the area of my research. This cannot be done without the great help provided by my supervisors. Thus, I would like to give my sincere thanks to my supervisor, Dr. Chengyuan Wang. Dr. Chengyuan Wang is a very respectful researcher, who has insights into my research topics. Therefore, with his crucial instructions, I have overcome challenges and difficulties one after another. More importantly, as a highly experienced researcher, he did not become impatient at my naive questions during the initial period of my $\mathrm{PhD}$ life. Instead, he always spent his precious time to explain everything to me patiently. It is stated that the character of your mentor plays a pivotal role in your experience of $\mathrm{PhD}$ life. Therefore, the noble qualities of my mentor made my experience as a PhD candidate superb. Sometimes, I felt that my mentor helped me pull through many excruciating difficulties just like a father. At this moment, due to the limitation of space, I can only express the countless grateful words in my heart as a sincere 'THANK YOU'. Meanwhile, I would like to give my sincere thanks to my second supervisor Prof. Perumal Nithiarasu. He provided very useful advice on my $\mathrm{PhD}$ study and is very patient to supervise the progress of my $\mathrm{PhD}$ study. Moreover, his understanding in science and engineering influenced me a lot. Overall, their excellent supervision has made my $\mathrm{PhD}$ study a valuable chance to increase my knowledge and expand my vision. It is such a great choice to learn from them, which I would cherish forever.

I would like to thank my wife, Chenyun Yu. She is the most important person in my life. I could not finish the thesis without her understanding and support. Her love for me could provide me with the endless power to overcome any difficulties in my life. During my $\mathrm{PhD}$ study, the conversations with her made me overcome the loneliness. In short, I cannot live without her. Also, I would like to thank my parents, who have been giving me their largest support since I was born.

I would also show my appreciation to Swansea and Swansea University for providing me with a peaceful place to do research. And I love this city far more than those big cities in this world because it can help me concentrate on my own works.

I would also like to thank Dr. Min Luo, Dr. Chenfeng Li and Dr. Yunqing Xuan for their friendly help and support during my PhD study. Also, I would like to thank the research equipment provided by the College of Engineering.

I would like to give my best wishes to my friends in Swansea University: Ruijie Wang, Yao Xiao, Jinsheng Wang, Jinlong Fu, Guitian Lai, Bin Chen, Shaoqin Cui, Qiao Wang, Yong Wang, Tingting Zhao, Jiabing Dong, Yan Shang, Zhi Li, Yanan Sun, Han Wang, Bingbing Chen, Xiaozhu Yu, Dawang Zhang, Sizeng You, Tongming Qu, Zheng Cui, Jiaying Zhang, Chen Wang, Shuo Deng, Gang Liu, Rui Liang, etc. The friendly atmosphere helps to accomplish the $\mathrm{PhD}$. I would like to express my thanks to my friends in China, the common interest made me feel happy to talk with them. 
I would like to thank the China Scholarship Council, and the College of Engineering of Swansea University, for providing me with the joint scholarship. The scholarship enables my study in the UK. I wish China and the UK could further establish closer links on the trade and culture despite their significant social and historical differences. 


\section{Contents}

Chapter 1 Introduction .............................................................................................................. 1

1.1 Brief review of CSK filaments........................................................... 1

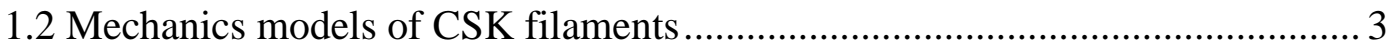

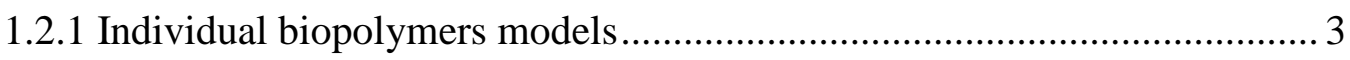

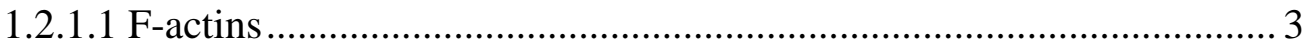

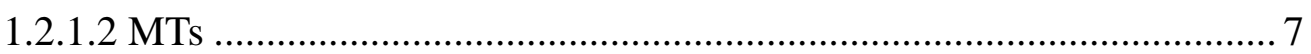

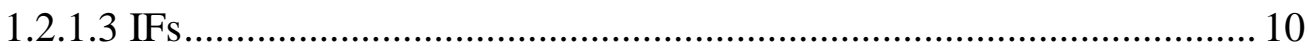

1.2.2 Subcellular structures models ........................................................... 10

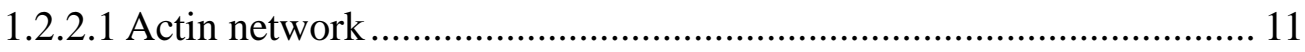

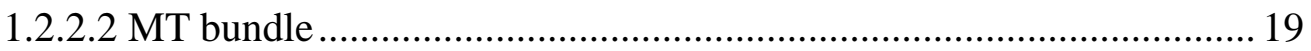

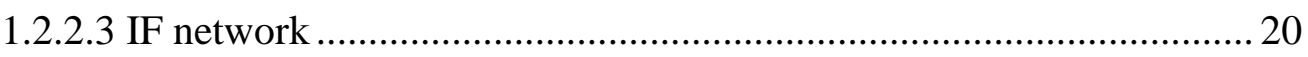

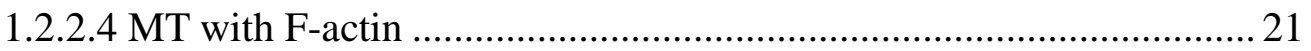

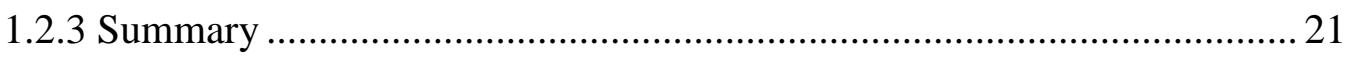

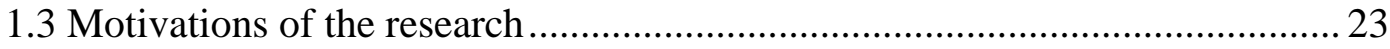

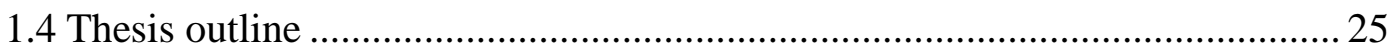

Chapter 2 Molecular structural mechanics models ...................................................... 28

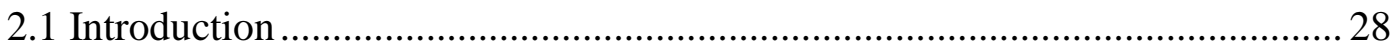

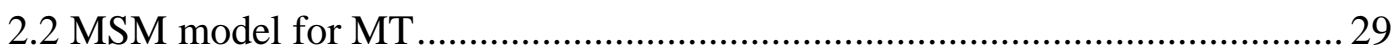

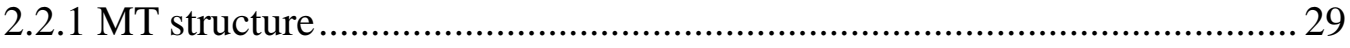

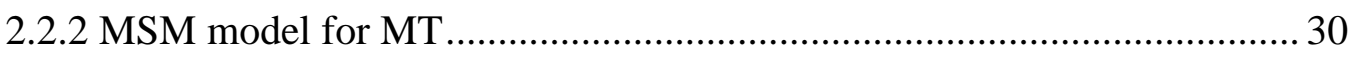

2.2.3 The mass matrix and stiffness matrix for MSM model............................ 33

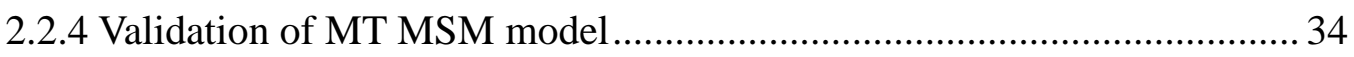

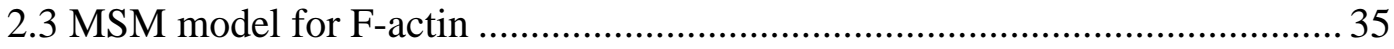

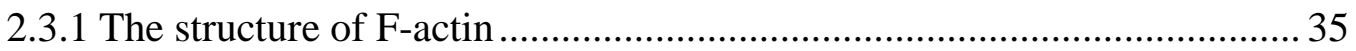

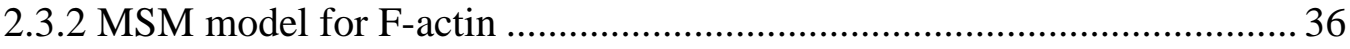

2.3.3 MD simulations on protein interaction .................................................. 37

2.3.4 Validation of F-actin MSM model ......................................................... 43

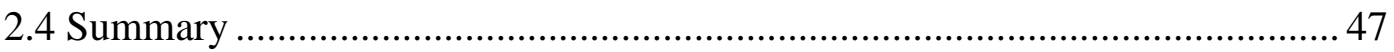

Chapter 3 Protofilament sliding and its effects on MT mechanics....................... 49

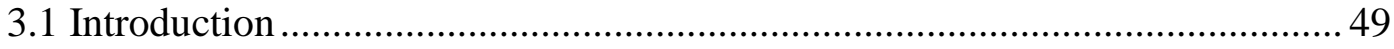

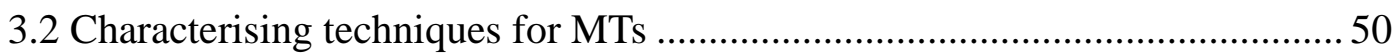

3.2.1 The vibration and static deformation of an MT with MSM model........... 50

3.2.2 Shear modulus and bending stiffness measurement.............................. 51

3.2.3 Nonlocal coefficient measurement ....................................................... 54 
3.3 A coupled molecular and continuum mechanics study for MT deformations . 55

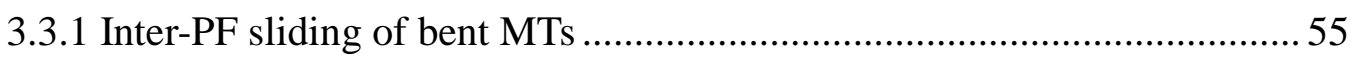

3.3.2 Classical beam models for MTs ............................................................. 58

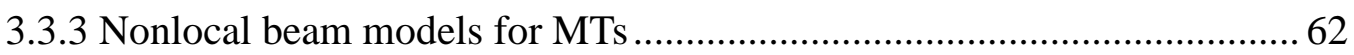

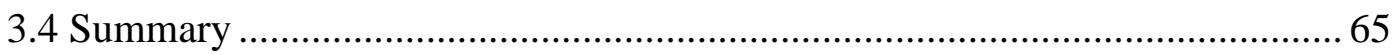

Chapter 4 Helix structure effects on the mechanics of CSK filaments ............... 66

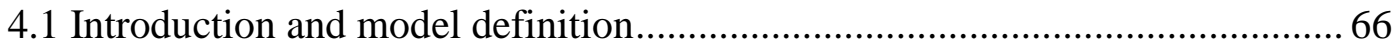

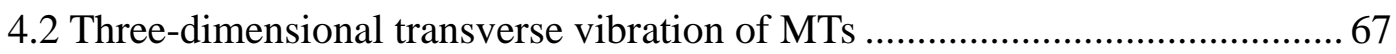

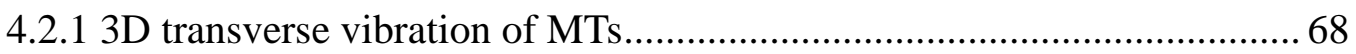

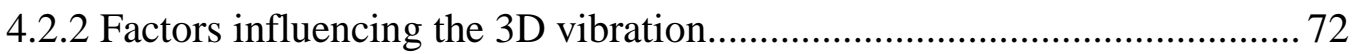

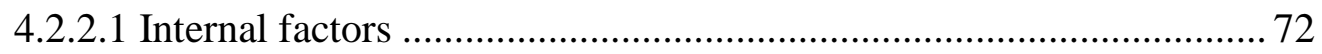

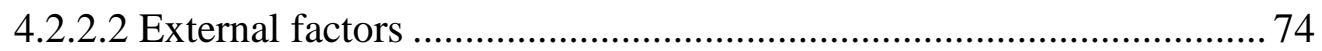

4.3 Tension induced bending in F-actin ...................................................... 77

Chapter 5 Subcellular environment effect on the filament mechanics.................81

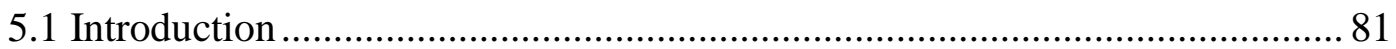

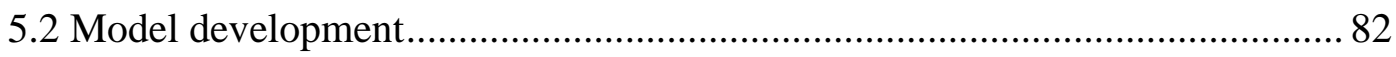

5.2.1 The details of the MSM model for buckling simulation .......................... 82

5.2.2 The model of the cross-linkers system supporting MTs ......................... 83

5.2.3 The Model of F-actin supported by the ABPs......................................... 86

5.3 Effects of the cross-linkers on the buckling of MTs in cells .......................... 87

5.3.1 Transition due to the addition of the 3D-linkers ................................... 87

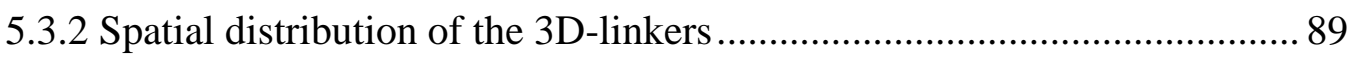

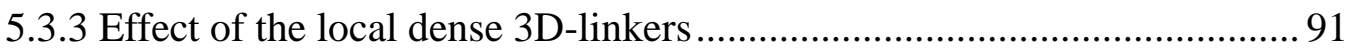

5.4 Effect of Binding Proteins on Filopodial F-Actin........................................ 93

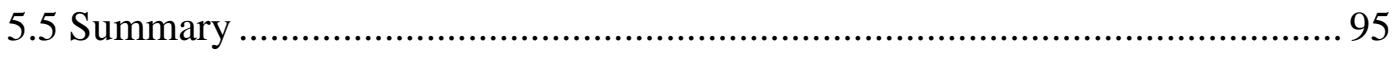

Chapter 6 Electromechanical vibration of CSK filaments....................................99

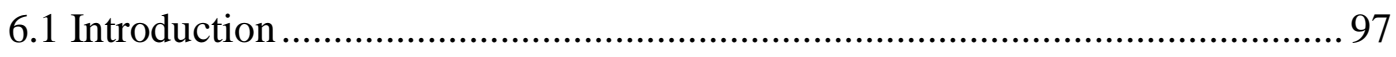

6.2 Electric excited vibration of MT and its application in biosensors................. 99

6.2.1 Modelling of excitation and damping of MT vibration ........................... 99

6.2.1.1 Modelling of EF excited vibration of MT ....................................... 99

6.2.1.2 Brief introduction to the preliminary evaluation of damping .......... 101

6.2.1.3 Supplementary details of the model.............................................. 102

6.2.2 Characterisation of the electromechanical vibrations ........................... 105

6.2.2.1 Vibrations excited by transverse electric field ................................ 105

6.2.2.2 Vibrations stimulated by axial electric field.................................. 107

6.2.3 Dependence of vibration on tubulin interaction.................................... 109 
6.2.4 Preliminary evaluation of reduced cytosol damping.

6.3 Effect of nanoscale solid-liquid interface on electromechanical vibration of

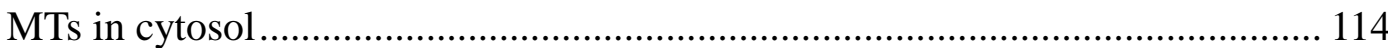

6.3.1 The MD simulation and the model development ................................... 114

6.3.2 The effect of nanoscale interface on MT vibration in electric field ........ 117

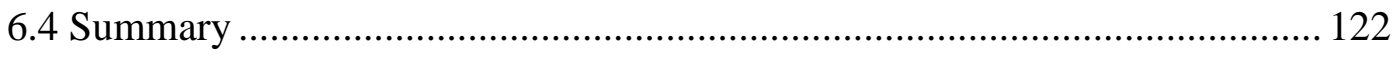

Chapter 7 Conclusions....................................................................................................... 124

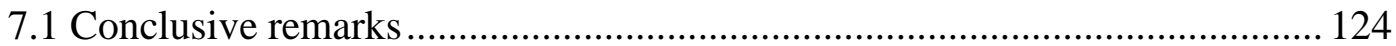

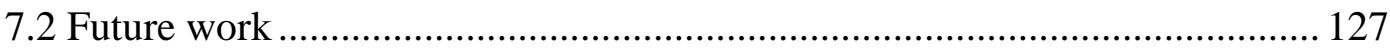

References ......................................................................................................................................... 130 


\section{List of figures}

Figure 1.1 (a) Microscopy illustrations of F-actin and MTs, and examples of schematic representations for (b) a Ratchet model of F-actin, (c) Molecular dynamics (MD)/ coarse-grained (CG)-MD model of F-actin, (d) a Shell model of MT and (e) an MD model of IFs

Figure 1.2 The examples of schematic representations for (a) cross-linked beams model of actin network, (b) a rigid rods model of actin network, (c) a bead-spring model of MT bundle and (d) a suspension bridge model of cross-linked MTs and F-actins

Figure 2.1 (a-c) Parameterized MT structure and (d) its MSM model; Here $a_{m}$ is the subunit repeat, $r_{m}$ is the subunit rise, $R$ is the radius of the MT, $\delta x$ is the PF separation and $\theta_{m}$ is the skew angle of PF relative to the direction of the axis of the MT. Vertical blue lines represent the intra-PF bonds of the MT and beam 1 of the MSM model, and the red ones denote the inter-PF bonds of the MT and beam 2 of the MSM model. (e) the deformation patterns of the tubulin bonds of the MT and (f) the space beams of the MSM model

Figure 2.2 (a) Structural representation of an F-actin with monomer interactions 1 and 2, (b) the MSM model developed for the F-actin with elastic beams 1 and 2 characterising interactions 1 and 2, respectively, the deformation patterns of (c) the monomer interactions of F-actin and (d) the elastic beams 1 and 2 of the MSM model

Figure 2.3 Schematics of an F-actin structure showing (a) numbering method of the monomers and the deformation patterns of the monomer interactions including (b) the interaction stretching and (c) interaction bending in the helical direction, and (d) the interaction stretching and (e) interaction bending in the longitudinal direction .38

Figure 2.4 Force-displacement relation obtained for Pulled-G in pulling direction for interaction stretching along (a) the helical and (b) the longitudinal directions, and interaction bending in (c) the helical and (d) the longitudinal directions

Figure 2.5 Experimental setup in the MSM simulations for (a) tensile, (b) torsion and (c) bending tests of a cantilevered F-actin . .44

Figure 2.6 The length dependence of (a) Young's modulus $Y$, (b) torsional rigidity $\kappa_{T}$ and (c) flexural rigidity (EI) calculated for F-actins with angular separation of $166.15^{\circ}$ and $167.14^{\circ}$, respectively

Figure 3.1 Experimental setup in the MSM simulations for (a) torsion, (b) the bending of a cantilevered MT subject to a distributed force, (c) the bending of a VIII 
cantilevered MT due to a concentrated load on the free end and (d) the vibration of a simply supported MT

Figure 3.2 The initial position of an undeformed 13-3MT and the final position of the bent MT structures with $\Omega=0.01,0.1,1,10$ and 100 , respectively. The illustrated displacements in the snapshots were enlarged 100-fold to reveal the differences. The inset shows the $\Omega$-dependence of the shear modulus $G$ (diamonds), the total bending deflection of the free end (squares), the deflection due to pure bending (circles) and the one resulting from the shear deformation (triangles). The deflections were given by the TB model by using the values of $G$ shown in the inset .56

Figure 3.3 The $\Omega$-dependency of the shear modulus $G$ (squares) obtained in the MSM simulations and that of $(E I)_{e q}$ calculated for MT structures with $L / D=40$. $(E I)_{e q}$ obtained for vibrating MTs based on the EB and TB models are represented by diamonds and circles, $(E I)_{e q}$ of bent MTs under a uniform load given by the EB and TB models are denoted by triangles and upside-down triangles, and the one for bent MTs subject to a concentrated load achieved by using the EB and TB models are represented by squares and circles, respectively 58

Figure 3.4 The $\Omega$-dependency of $(E I)_{e q}$ obtained for bent MT structures which are subject to a distributed transverse load and possess $L / D$ rising from 10 to 320 .61

Figure 3.5 The $\Omega$-dependence of $\left(e_{0} a\right)^{2}$ calculated for 13-3MT structures. The data obtained for the vibrating MT structures based on the nonlocal EB and TB models are represented by squares and circles, respectively, and those for the bent MTs by using the nonlocal EB and TB models are denoted by triangles and the upside-down triangles, respectively. Negative values of $\left(e_{0} a\right)^{2}$ shown in the figure indicate the situation where the nonlocal beam model is not relevant for the mechanics of MTs

Figure 4.1 Transverse vibration modes $(m=4)$ and its the projection to the YOX, YOZ, XOZ planes obtained for (a) an imaginary non-helical 13 MT and (b) a 13-3 MT;

Figure 4.2 The simplified mode shape $(m=4)$ for (a) an imaginary 13MT and (b) a 13-3 MT where the rotation of the half wavelength planes (or oscillation planes) is shown. The real mode shape of the 13-3MT is shown in (c) where the bending axis rotates throughout the whole length of the MT 70

Figure 4.3 The material distribution on the perimeter of MTs with different $S$ .72

Figure 4.4 Dependence of the rotation angle $\xi_{\text {all }}(L=2000 \mathrm{~nm})$ on (a) the helical start number $S$ and (b) the number of protofilaments $N$ obtained for the 4th model of the MT vibration .73 
Figure 4.5 The effect of structural details on the frequency of MTs with different $S$ and $N$. Here the half wave number $m$ changes from 1 to 4

Figure 4.6 The length dependence of the angle $\xi_{\text {all }}$ of standard 13-3 MTs .75

Figure 4.7 Mode shapes of a vibrating MT with (a) fixed-fixed ends and (b) fixed-free ends; The YOX projections of the mode shapes are shown in (c) and (d), respectively . .77

Figure 4.8 The tension induced bending of F-actin .78

Figure 4.9 Boundary condition effect on F-actin stretching .79

Figure 5.1 (a) The ideal individual MT buckling mode and the MT buckling observations in experimental study, (b) Structural representation of an MT and the MSM model, and (c) the structure of the cross-linker system supporting MTs.

Figure 5.2 The length-dependent Fcr calculated for individual MTs in comparison with the experimental data

Figure 5.3 (a) F-actin bundle in the protrusion of the leading edge in motile cells, and (b) the model of F-actin supported by ABPs in filopodia protrusion

Figure 5.4 The 3D buckling modes of the selected MT-linker systems with randomly generated cross-linkers .88

Figure 5.5 The effect of the spatial linker orientations obtained by considering a 2D-linker system and the systems with increasing amount of the 3D-linkers ....

Figure 5.6 The effect of the spatial linker distributions on the critical buckling load obtained by considering the ' $\mathrm{n}$-repeat' linkers

Figure 5.7 The effect of the local dense linkers on the buckling modes and associated critical loads

Figure 5.8 (a) The actin bundle which generates force for protrusion of the leading edge in motile cells and (b) Length-dependence of the critical buckling forces $N_{c r}$ obtained for the F-actins supported by ABPs with different extensional stiffness .93

Figure 6.1 Illustration of an individual MT and an MT-cytosol system subject to an electric field generated by a dipole antenna

Figure 6.2 The comparison between the Eigenvalue transverse vibration modes and the vibrations of MTs excited by the EF in the transverse direction 106 
Figure 6.3 The vibration modes of MTs excited by the EF in the axial direction ...108

Figure 6.4 The changes in vibrational responses and elastic moduli of MTs as a result of (a) abnormal longitudinal interactions between tubulins and (b) abnormal helical interactions between tubulins

Figure 6.5 The changes in responses of damped MT vibration in cytosol due to different damping reduction factors

Figure 6.6 (a) The illustration of the interface between MT and cytosol (inset: simulation box); (b) the dimer region within which the distance between water molecules and dimer atoms are measured; (c) the histogram of the distances measured between the dimer and water atoms; (d) the simulations on the relation between the water-dimer distance and their interaction potential energy; (e) interface potential energy $E$ calculated as a function of the water-dimer distance change $\Delta d$; (f) the physical model for the nanoscale interface between MT and cytosol (case 3);.115

Figure 6.7 The two types of MT-cytosol interfaces models 116

Figure 6.8 The amplitude-frequency spectrum of (a) individual MT (case 1) subject to a TEF, (b) MT-cytosol system subject to a TEF with a non-slip (case (2)) or a nanoscale interface (case (3)), (c) individual MT subject to an AEF and (d) MT-cytosol system subject to an AEF with a non-slip (case (2)) or a nanoscale interface (case (3)) 118

Figure 6.9 Time dependent amplitude of MT vibration excited by a TEF at frequency (a) $1 \mathrm{MHz}$ and (b) $18 \mathrm{MHz}$, respectively, and the vibration stimulated by an AEF at (c) $1 \mathrm{MHz}$ and (d) $53 \mathrm{MHz}$, respectively .121 


\section{List of tables}

Table 2.1 The structural details of various MTs ......................................................31

Table 2.2 The force constants of interactions between monomers of F-actin .........43

Table 4.1 Natural frequencies of different vibration modes of models (MHz) ..........69

Table 5.1 Comparison to the cross-linked 1D-FE model .........................................85 


\section{Nomenclature}

\section{Symbols}

$a_{m}$

$r_{m}$

$R$

$\delta x$

$\theta_{m}$

$N$

$S$

$U_{i}^{r}$

$U_{i}^{\varphi}$

$U_{i}^{\tau}$

$\Delta r_{i}$

$\Delta \varphi_{i}$

$\Delta \Phi_{i}$

$k_{i}^{r}$

$k_{i}^{\varphi}$

$k_{i}^{\tau}$

$U_{i}^{A}$

$U_{i}^{M}$

$U_{i}^{T}$

$\Delta l_{i}$

$\Delta \alpha_{i}$

$\Delta \beta_{i}$

$Y_{i} A_{i}$

$Y_{i} I_{i}$

$S_{i} J_{i}$

$l_{i}$

$M_{\text {mono }}$

$\theta_{a}$

$D_{a}$

$r_{a}$

$\kappa^{T}$

Y

EI

G

L

subunit repeat of MT

subunit rise of MT

radius of the MT

PF separation of MT

skew angle of PF relative to the direction of the rolling axis of MT

$\mathrm{PF}$ number of MT

helix-start number of MT

bond stretching energy in MSM ( $i$ denotes the types of bonds/beams in MSM)

bond angle bending energy in MSM

bond dihedral angle torsional potential energy in MSM change of bond length in MSM change of in-plane bond angle in MSM

change of out-of-plane angle in MSM

force constant for bond stretching in MSM

force constant for bond angle bending in MSM

force constant for bond torsion in MSM

strain energies due to beam tension in MSM

strain energy due to beam bending in MSM

strain energy due to beam torsion in MSM

length change of the beam in MSM

bending angle of the beam in MSM

torsion angle of the beam in MSM

extensional stiffness of the beam in MSM

bending stiffness of the beam in MSM

torsional stiffness of the beam in MSM

length of the beam in MSM

mass of monomer in MSM

angular separation in F-actins

diameter of F-actin

subunit rise of F-actin

torsional rigidity

Young's modulus

flexural rigidity

Shear modulus

length of filament 


$\begin{array}{cc}w & \text { transverse deflection } \\ \Omega & \text { coefficient alters the effect of the inter-PF sliding } \\ e_{0} a & \text { nonlocal effect coefficient } \\ A_{0} & \text { area of cross section of MT } \\ K_{\mathrm{S}} & \text { shear correction coefficient } \\ F_{c} & \text { concentrated transverse force } \\ q_{0} & \text { uniformly distributed transverse force } \\ m & \text { axial half wave number } \\ \xi & \text { rotation angle between oscillation planes in MT vibration } \\ L_{d} & \text { distance between the adjacent linkers for MT } \\ k & \text { spring constant } \\ F_{c r} & \text { critical buckling load of MT } \\ N_{c r} & \text { critical buckling load of F-actin } \\ F_{d} & \text { damping force on the MT monomers } \\ P & \text { damping reduction coefficient in damped MT vibration } \\ Q & \text { quality factor for the damped MT vibration } \\ h_{s} & \text { width of frequency range of the peak in vibration spectrum } \\ W_{f r} & \text { with amplitude greater than a given value } \\ \Delta d & \text { change in the distance between the dimer and the water }\end{array}$

$\begin{array}{cc} & \text { Abbreviations } \\ \text { CSK } & \text { Cytoskeleton } \\ \text { MT } & \text { Microtubule } \\ \text { F-actins } & \text { Actin Filaments } \\ \text { CSK filament } & \text { Cytoskeletal filament } \\ \text { IFs } & \text { Intermediate Filaments } \\ \text { BD } & \text { Brownian Dynamics } \\ \text { MD } & \text { Molecular Dynamics } \\ \text { SMD } & \text { Steered MD } \\ \text { CG } & \text { Coarse-Grained } \\ \text { ECM } & \text { Extra Cellular Matrix } \\ \text { WLC } & \text { Wormlike Chain } \\ \text { WLB } & \text { Worm Like Bundle } \\ \text { PFs } & \text { Protofilaments } \\ \text { NMA } & \text { Normal-Modes Analysis } \\ \text { MSM } & \text { Molecular Structural Mechanics } \\ \text { SSM } & \text { Substructure Synthesis Method } \\ \text { SFs } & \text { Stress Fibres } \\ \text { KVM } & \text { Kelvin-Voigt-Myosin }\end{array}$




$\begin{array}{cc}\text { ABP } & \text { Actin Binding Protein } \\ \text { EF } & \text { Electric Field } \\ \text { CMM } & \text { Continuum Mechanics Model } \\ \text { ANEM } & \text { Anisotropic Elastic Network Model } \\ \text { MAP } & \text { MT-Associated Protein } \\ \text { PME } & \text { Particle Mesh Ewald } \\ \text { EB } & \text { Euler Beam } \\ \text { TB } & \text { Timoshenko Beam } \\ \text { FE } & \text { Finite Element } \\ \text { 1D } & \text { One Dimensional } \\ \text { 2D } & \text { Two Dimensional } \\ \text { 3D } & \text { Three Dimensional } \\ \text { vdW } & \text { Van der Waals } \\ \text { CNT } & \text { Carbon Nanotube } \\ \text { RBM } & \text { Radial Breathing Mode } \\ \text { Pulled-G } & \text { The Group of Pulled Atoms } \\ & \text { in SMD Simulations } \\ \text { Fixed-G } & \text { The Group of Fixed Atoms } \\ \text { TEF } & \text { in SMD Simulations } \\ \text { AEF } & \text { Transverse Electrical Field } \\ \text { RBV } & \text { Axial Electrical Field } \\ & \text { Radial Breathing Vibration } \\ & \\ & \end{array}$




\section{List of publications}

[1] Li, S.; Wang, C. Y.; Nithiarasu, P., Three-dimensional transverse vibration of microtubules. Journal of Applied Physics 2017, 121 (23), 234301.

[2] Li, S.; Wang, C.; Nithiarasu, P., Structure-property relation and relevance of beam theories for microtubules: a coupled molecular and continuum mechanics study. Biomechanics and modeling in mechanobiology 2018, 17 (2), 339-349.

[3] Li, S.; Wang, C.; Nithiarasu, P., Effects of the cross-linkers on the buckling of microtubules in cells. Journal of Biomechanics 2018, 72, 167-172.

[4] Li, S.; Zhang, J.; Wang, C.; Nithiarasu, P., Atomistic Modeling of F-Actin Mechanical Responses and Determination of Mechanical Properties. ACS Biomaterials Science \& Engineering 2018, 4 (8), 2794-2803.

[5] Li, S.; Wang, C.; Nithiarasu, P., Electromechanical vibration of microtubules and its application in biosensors. Journal of the Royal Society Interface 2019, 16 (151), 20180826.

[6] Li, S.; Wang, C.; Nithiarasu, P., Simulations on an undamped electromechanical vibration of microtubules in cytosol. Applied Physics Letters 2019, 114 (25), 253702. 


\section{Chapter 1 Introduction}

\subsection{Brief review of CSK filaments}

Cells are the fundamental component of tissue. Their dynamic, living feature endowed them with the ability to change in accordance with their functional state and response to stimuli within the environment [1]. The cytoskeleton (CSK) is a structure that enables cells to maintain their shape and internal organization. The mechanical support provided by CSK enables cells to perform essential functions such as division and motility. The CSK is a network of several different subcellular components [2]. The basic subcellular components are three types of filamentous proteins, i.e., microtubules (MTs), actin filaments (F-actins) and intermediate filaments (IFs). MTs are the largest filament, with a diameter of about $25 \mathrm{~nm}$, and they are composed of a protein called tubulin. F-actins are the smallest type, with a diameter of only about $7 \mathrm{~nm}$, and they are made of a protein called actin. IFs, as their name suggests, are mid-sized, with a diameter of about $10 \mathrm{~nm}$. Unlike F-actins and MTs, IFs are constructed from a number of different subunit proteins. Along with these three major classes of filaments, there are a host of adaptors, regulators, molecular motors, and additional structural proteins [3] which allow the CSK to form various subcellular structures. Although distinct in their properties and the types of network they form, the filaments of the CSK are intricately linked together [4]. The organization of these links and the resultant architecture of the CSK networks plays an important role in cell mechanics [4]. Scaffold formed by MTs, F-actins or IFs interact with each other and other subcellular structures either nonspecifically (through steric interactions and entanglement) or specifically (through proteins that link one filament type to another) [4]. This interconnectivity creates continuous mechanical coupling through the CSK, providing a means for internal or external forces and fluctuations to be distributed throughout the cell [4]. It is also worth mentioning that there are different cell types and each type of cells is specialised to 
carry out particular functions which involve the biological processes associated with the CSK filament mechanics. For instance, in muscle cell, F-actins and myosin filaments allow muscle contraction by sliding past one another [5-7]. The production of mechanical force in muscle is believed to be the result of a dynamic interaction between the proteins [5-7]. And in neurons, one of the major intracellular transport systems is the MT-based transport system along which kinesin and dynein motor proteins generate force and drive the traffic of many cellular components [8]. In epithelia, keratins IFs can form junctions that hold cells together (desmosomes), or attach cells to matrix (hemidesmosomes) [9].

Since many biological processes depend crucially on the characteristics of CSK and their components (e.g., materials properties, architecture, dynamics, etc.), the experiments have been conducted with their focuses on CSK mechanics $[1,10]$. The experiments are important for knowing how much we can probe the CSK with current characterisation techniques. For instance in the researches for measuring the flexural rigidity of individual filaments [11], fluorescence microscopy was utilised to record the fluctuating shapes of the microtubule. Also in measuring the mechanical properties of F-actins, optical/electron microscopy and fluorescence light microscopy were proven to be useful in capturing the thermal fluctuation shapes [11-15]. Moreover, other experimental techniques to measure the mechanical properties of individual filaments were also reported, to name a few, the optical diffraction method [16], polarized fluorescence [17] and optical trapping technique [18]. The application of the optical diffraction method on the electro-optic effect of F-actin in muscle fibres can reveal the bending mode and estimate the flexural rigidity of the filament [16]. The polarized fluorescence and micro-needle technique can probe the force and displacement produced by single heads in synthetic myosin filaments interacting in the correct orientation with the actin filament [17]. With an optical trap, the force on single F-actins in a motility assay can be measured [19]. Also with an optical trap, the torsional rigidity can be measured directly by visualizing the torsional Brownian 
motion of a single filament [18]. Moreover, extremely precise manipulation of the filament can be achieved with the atomic force microscopy, which can yielded a shear modulus that was 2-3 orders of magnitude smaller than the Young's modulus of the filaments $[20,21]$. And in identifying the role of the larger scale filamentous system of actin cortex in regulation of cell surface tension, the scanning electron microscope can be used to characterise the cortices of different stages of cell [22].

Those experimental works have also offered an extensive knowledge of the mechanical properties of cells, as well as their response to different chemical and mechanical stimuli [1]. Based on these research outcomes various in-silico mathematical frameworks have been developed to interpret the data collected in experiments and provide insights on cell mechanics. The following section gives an overview of the development history of the mechanics models for CSK filaments and other subcellular structures.

\subsection{Mechanics models of CSK filaments}

\subsubsection{Individual biopolymers models}

In this section, the mechanics models achieved so far for the three main component filaments in CSK, i.e., MTs, F-actin and IFs, will be reviewed in terms of the development history and the major research focuses. The microscopy illustrations of the F-actins and MTs and their distribution in cell was provided in Fig. 1.1(a) [23]. It is seen from Fig. 1.1(a) that the length scale of the filaments are similar to the scale of cell ( $\mu \mathrm{m}$ scale) but the diameters of the filaments are in nanometre scale which is far less than the cell size.

\subsubsection{F-actins}

F-actins are long filamentous polymers acting as basic structural components in eukaryotic cells $[24,25]$. Owing to the unique molecular structures, F-actins exhibit the ability to perform a broad range of essential cellular functions in cell motility as 
well as in locating and transporting protein complexes in cells [26].

Other than the basic continuum mechanics theory in organizing the experimental results of F-actins [11], the ratchet model (Fig. 1.1(b)) was developed by Peskin et al. in 1993 [27] to describe the force generation during the cellular protrusion by actin polymerization. The ratchet model is an active model based upon the idea that chemical reactions generate cellular protrusive forces during F-actin and MT polymerization, via rectifying Brownian motion. Few years later, Mogilner and Oster [28, 29] generalized the rigid polymerization ratchet theory. This model was developed for rectified diffusion to the situation when the polymerizing filaments are flexible, so that their thermal fluctuations are sufficient to create a monomer-sized gap to permit polymerization. The model is able to identify the dependency of the load-velocity relationship on the length of the filaments and the magnitude of the applied force. Later, to better account for the rapid elongation and substantial force generation by CSK filaments, the modified models such as the end-tracking motor model [30] were developed. In the end-tracking motor model, the filament end-tracking proteins advance on filament ends and facilitate rapid elongation and substantial force generation by persistently tethered filaments.

Until 1995, analyses of the mechanical properties of actin have largely relied on simple homogeneous cylinders models. To study the unique features of 3D atomic structure (e.g., motions and flexibilities), a normal-modes analysis (NMA) model [31] was developed to analyse the dynamical spectrum of F-actin. The model considered a short segment of F-actin with a highly simplified representation without considering any internal flexibility within the actin monomer. The simulation based on the model proceeds from the atomic level. Relying on a single fitting parameter, it can reproduce many experimental data, such as persistence lengths, elastic moduli, and contact energies.

In 1996, The wormlike chain (WLC) model was utilized to characterise e.g., thermal fluctuation of F-actin $[32,33]$. In the WLC model the polymer is considered 
as a differentiable space curve and the conformations of polymer chains is quantified by quantifying distribution function of end-to-end distance.

As reviewed above, effort has been devoted to studying the growth, mechanics and conformations of F-actins. In 2001, the interest of investigation was transferred to the process of spontaneous nucleation. Sept and McCammon explored the thermodynamics and kinetics of F-actin nucleation with a Brownian dynamics (BD) model [34]. The basis of the BD simulations is the solution of the equation that relates position of the protein, diffusion constant, time step and temperature. The model was applied to find out the binding free energies of the structures along the nucleation pathway in this study. Furthermore, the BD models were also used to investigate the remodelling processes of actin dynamics [35].

(a)

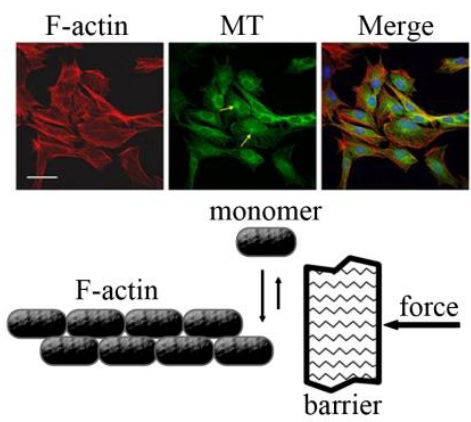

(d)

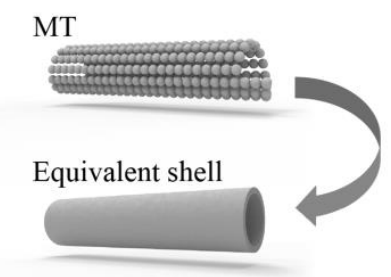

(c)

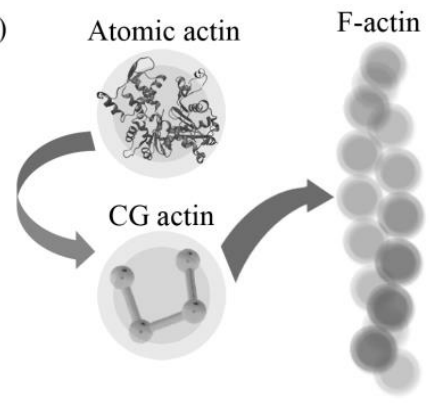

(e)

$$
\text { Atomistic model for IFs }
$$
in MD simulation

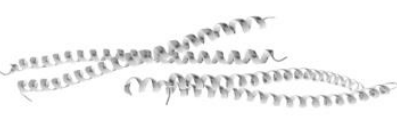

Figure 1.1 (a) Microscopy illustrations of F-actin and MTs [23], and examples of schematic representations for (b) a Ratchet model of F-actin [27], (c) Molecular dynamics (MD)/ coarse-grained (CG)-MD model of F-actin [36], (d) a Shell model of MT [37] and (e) an MD model of IFs [38]

To overcome the size limit of existing models for F-actins, Ming et al. [39] developed an analytical method called the substructure synthesis method (SSM) in 2003. The basic concept of the SSM model is to treat the motions of a given structure as a collection of those of an assemblage of substructures. The choice of substructures is arbitrary and sometimes quite natural, such as domains, subunits, or 
even large segments of biomolecular complexes. The authors applied the SSM technique to analyse the vibration of F-actins whose lengths ranged from 2 to $2^{7}$ substructures $(4.6 \mu \mathrm{m})[40]$.

In 2005, it was realised that the existing models were unable to delineate the effect of detailed structures in subdomains of actin on structural and mechanical properties of F-actins. Therefore, Chu and Voth [36, 41] utilized the molecular dynamics (MD) simulation and the coarse-grained molecular dynamics (CGMD) method to examine the influences of atomic monomer conformation (Fig. 1.1(c)). In the most MD simulations, the trajectories of atoms and molecules are determined by numerically solving Newton's equations of motion for a system of interacting particles, where forces between the particles and their potential energies are often calculated using interatomic potentials or molecular mechanics force fields. CG models could provide a promising approach to increasing the timescale/scale by treating small groups of atoms as single particles. In Chu's study, CG actin molecules were joined to construct the filament model with a temporal scale at which their molecular structure changes according to atomic motion is negligible during the investigation into characteristics such as macroscopic stiffness. The model is able to determine the persistence length of F-actin and relate the conformation of the DNase I-binding loop and the intermonomer interactions. Several years later, the MD simulation was further utilized by Matsushita et al. [42] to evaluate the extensional and torsional stiffness of F-actin. It is worth mentioning that a multi-scale model with the combination of MD and NMA was also developed by Deriu et al. [43] to study the relationship between the mechanics of F-actin and its molecular architecture. Moreover, the CGMD method was also applied in subsequent studies of F-actin to identify the heterogeneous mechanical properties of F-actin due to the difference in G-actin subunit structures [44-46].

In addition to the models mentioned above, models with particular considerations were developed, able to explore mechanical behaviour of actin CSK 
at the fundamental filament-level. For instance, in 2010 the Cosserat continuum model of an F-actin proposed by Yamaoka and Adachi [47] considered the F-actin as an elastic rod with mismatch between centroid and central axis. The mismatched structure may lead to the coupling between the axial stretch and bending deformation as well as the connection between the axial stretch and twisting deformation. Very recently a molecular structural mechanics (MSM) model for F-actin has been developed by Li et al. [48]. This model is used to characterise the reinforcing effect of the actin-binding proteins (ABPs) on single F-actin during filopodial protrusion. The model could describe the nanoscale protein interactions. And it enjoys the highly improved efficiency and the expanded scope of the research as compared with formidable MD simulations and difficult nanoscale experiments. However, it is expected that the non-linear large deformation behaviour of the F-actin could be better addressed in the future version of the model.

\subsubsection{MTs}

MTs are a structural element and primary organizer in the CSK of eukaryotic cells [49]. They form "tracks" on which motor proteins transport organelles and construct the spindle apparatus to facilitate cell division [50]. They are also responsible for maintaining the shape and providing the rigidity of the cells.

Similar to the research focus on the force generation during the cellular protrusion by actin polymerization, the force-velocity relationship during the polymerization of MTs also attracted the attention of early researchers. In 1997 Dogterom and Yurke [51] measured the force-velocity relation generated by a single MT and described the mechanism by the polymerization ratchet model [27]. Later, the Ratchet model was further generalized by Mogilner and Oster [52] to delineate the growth of MTs to take into account the "subsidy effect" that arises because an MT consists of 13 protofilaments (PFs). The model is able to predict the length dependent growth rate and force of MTs and estimate the MT driven deformation of 
liposomes observed in experiments and features of filopodial protrusion.

In terms of the other mechanical issues of MTs, such as the simulations of vibration, buckling and thermal fluctuation of MTs, the researchers would always take the structure of the MTs into consideration. Chrétien et al. [53, 54] have given precise descriptions of MT structures in terms of a surface lattice composed of tubulin monomers in the lattice accommodation model. Inspired by the lattice model, a two-dimensional lattice model for MT vibration was developed by Portet et al. [55] in 2005. It considers dimers as points at their centres of mass. The pitch of helices that run around the MT wall is proportional to the equilibrium distance between adjacent dimers along a PF. The model is able to make predictions regarding the vibration dispersion relations as well as vibration propagation velocities, and it could relate the vibration frequencies and velocities with the elastic constants and the geometrical characteristics of the MTs.

Also, based on the assumptions that the MTs could be equivalent to continuum structures, the continuum mechanics theory was widely used to explain the experimental results of MTs [11, 21, 56]. Theoretical investigations were also conducted in studying the natures of MTs which the experimental techniques have limited power to provide insights. To characterise the acoustic vibration behaviour of MTs, a continuum shell model was developed by Sirenko et al. [57]. The MTs were modelled by thin elastic cylindrical membrane shells, and it is able to derive the eigenfrequencies and eigenmodes of the confined elastic vibrations in a shell-fluid system. Then the continuum mechanics model (CMM) experienced a further development by the work of Ru's group, including the shell model (Fig. 1.1(d)) [37, 58-61] and the beam model [62]. The beam model for MT can also be found in [63, 64]. These models are capable of characterising various features of MTs such as non-local effect and buckling. It is worth mentioning that there also exist other CMMs for unique mechanical features of MT such as size-effect for buckling [65-68]. 
However, the efficient CMMs are oversimplified in view of the detailed molecular structure of monomers of MTs, which could exert influence on the properties of MTs. To overcome this limitation, the MD simulation technique was applied by Deriu et al. [69] to characterise the monomeric information of MT and a mesoscale spring model was proposed based on the information obtained. Four years later, based on Deriu's work and additional information, Ji and Feng [70, 71] further developed CG mechanochemical model to study the dynamic behaviour of MT. The interactions among tubulins are taken into account from the molecular basis. The model is able to characterise the conformations of sheet-ended MTs, the structural evolution and to further simulate the radial indentation process of an MT. It is worth mentioning that similar to the history of the MD models for F-actin, an anisotropic elastic network model (ANEM) with the combination of MD and NMA was also developed by Deriu et al. [72] to study the MT mechanics. This technique could enjoy higher computational efficiency compared to MD and describe systems of hundreds of interacting tubulin monomers. Thus the MT macroscopic properties such as bending stiffness, Young modulus, and persistence length could be estimated.

Moreover, the WLC model mentioned for F-actins could also be applied to MTs. In 2008, the WLC model was utilized by Taute et al. [73] to address the dynamics of thermal shape fluctuations of MTs. The worm-like bundle (WLB) model [74] could also describe the MTs, which explicitly accounts for the discrete character of the internal architecture of the MT as a bundle of PFs, and its internal deformation modes. Unlike WLC, the model could exhibit a state-dependent bending stiffness that derives from a generic competition between the bending and twist stiffness of individual filaments and their relative motion mediated by the stiffness of the crosslinkers. The model is able to predict the relationship between the effective bending rigidity of MTs and the number of PFs.

Later other models for MTs were also developed to take more detailed features of MTs into consideration at lower computational cost, such as the 
atomistic-continuum model and the MSM model. In 2011, an atomistic-continuum model was developed for global buckling of MTs, which was also used to study MT vibration $[75,76]$. In the atomistic-continuum method, material properties and continuum energy are evaluated based on atomic interactions. It has the ability to overcome computational size limitations without losing inter-atomic information. In 2014, the MSM model was applied to MT to study the mechanical properties [77]. The MSM technique can establish the equivalence between the deformation of the molecular structure of MTs and the deformation of structural frame representation of MTs, and it is able to extract the materials information from MD simulation. The robustness and efficiency of the MSM model have been demonstrated in studying the different mechanical feature of MTs such as vibration and buckling [56, 78-82].

\subsubsection{IFs}

Compared with the various models on MTs and F-actins, the modelling effort on exploring another major component of the CSK in eukaryotic cells, IFs, is relatively scarce. IFs, are crucial in defining key biomechanical functions of cells such as cell migration, cell division and mechanotransduction, and have also been referred to as the "safety belts of cells" to prevent exceedingly large cell stretch [83-85]. The MD and CG models (Fig. 1.1(e)) were employed by Qin et al. [38] to give a quantitative comparison to experimental results of the IF nanomechanics. The model is able to probe the response of the IF under mechanical tensile stretch. And it enables a new paradigm in studying the biological and mechanical properties of IFs from an atomistic perspective, and lays the foundation to understanding how properties of individual protein molecules can have profound effects at larger length-scales.

\subsubsection{Subcellular structures models}

In this section, the models of the subcellular structures which are composed of interconnected individual filaments (MTs, F-actin and IFs) will be reviewed. It is 
clear that in understanding cell mechanical properties, it is essential to have a good representation of the subcellular structures such as the actin networks [86].

\subsubsection{Actin network}

Regarding the subcellular structures, major concern of the modelling studies has been placed on actin networks. Actin networks are networks of cross-linked and bundled F-actins[87]. They have numerous functions within the context of cell mechanics and the CSK, such as the pivotal role in motility and mechano-protection $[87,88]$.

During the early days of modelling actin network, the energy description was favoured by the researchers. In 1995, MacKintosh et al. [89] developed an energy-based model for crosslinked gels and sterically entangled solutions of semiflexible biopolymers to explain the elastic properties of in vitro actin networks, and successfully showed that the elastic properties of network depend on the concentration of actin solution. One year later, also with an energy description of biopolymer, Kroy and Frey [90] used a WLC model to find out the entanglement transition and plateau modulus of actin networks. The WLC description was used by Gardel et al. [87] in 2004 to delineate the role of entropic effects in network elasticity and to connect the mechanical properties of individual filaments with the elastic behaviour of network. Also by applying the WLC description, in 2008 Palmer and Boyce [91] were able to propose a detailed microstructurally-informed CMM and capture the stress-strain behaviour of the network. Later in 2011, with the help of the WLC description, Broedersz and MacKintosh [92] were able to generate a phantom network model to study the effects of motor generated forces on the mechanics of networks. Then in 2013, following the approach of Palmer and Boyce [91], a micro-sphere model for cross-linked actin network was developed by Unterberger et al. [93] with the support of the well-known WLC model describing single filament properties. The model is able to build a stable framework for solving more complex boundary-value problems such as the simulation of the indentation of an AFM 
cantilever tip into a surface.

When subjected to deformation, the actin networks will experience strain hardening responses and enhance the energy needed for further deformation [94]. Such strain hardening responses could be characterised by the early proposed open cell foam model [95]. In 1997, with an open cell foam model [96, 97], Satcher et al. [98] described the deformation of actin networks induced by filament bending twisting and sliding. In this model, the actin network is described as a rigid cross-linking of beamlike structures, of which the shape is either cuboid, dodecahedron, tetrakaidecahedron, or icosahedron, with bending and twisting of the struts as the major stress-generating component. With this model, the mechanical properties of networks could be computed and compared to the experimental results, and the model is also able to predict the strain hardening under compression for the adherent cells exposed to local mechanical perturbations [95]. The models for strain-stiffening characteristics of actin networks experienced further development in terms of the features like non-affine deformation. In 2007, Huisman et al. [86] presented the first computational studies of the large-strain mechanical response of discrete, 3D networks of cross-linked F-actin (Fig. 1.2(a)). The networks are generated by a procedure inspired by MD, after which they are deformed using an updated-Lagrangian finite-element model of beams. The model is able to demonstrate the relationship between the 3D network architecture and the non-affine behaviour of network. Then in 2008, Åström et al. [99, 100] developed an elastic network model using Euler-Bernoulli beam to describe the segments, and using springs to describe the cross-links at intersecting locations. By applying simulations with stiffness matrix method, complicated behaviour of network could be explored, including strain hardening, avalanches of cross-link slippage and spontaneous formation of stress-carrying fibre bundles. In combination with the WLC model, Huisman et al. [101-104] used a Monte Carlo scheme to generate thermalized networks. Starting from a random, isotropic network, Monte Carlo moves that alter 
the topology of the network are performed to minimize the free energy of the network. Subsequently, segments are cut until an average filament length is obtained, which results in a disordered network of curved filaments. The model can explore the

(a)

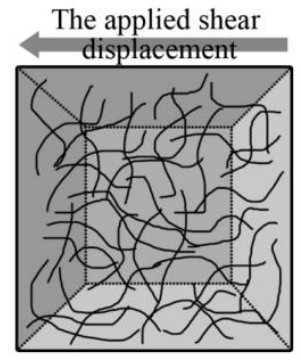

(c) (b)

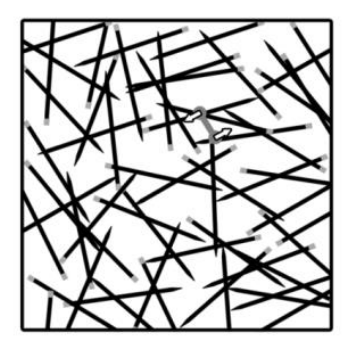

(d)
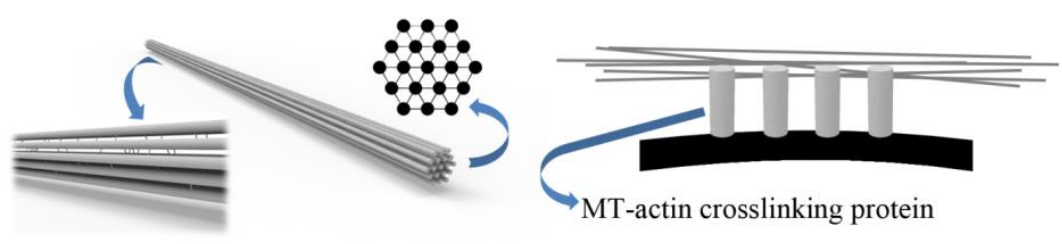

Figure 1.2 The examples of schematic representations for (a) cross-linked beams model of actin network [86], (b) a rigid rods model of actin network [105], (c) a bead-spring model of MT bundle [106] and (d) a suspension bridge model of cross-linked MTs and F-actins [68]

unique characteristics of network such as nonlinear stiffening behaviour and non-affine bending regime. Later a CG Monte Carlo model for F-actin network was utilized by Kang et al. [107] to simulate the response of network under cyclic stretching. The model is in principal suited to understanding how mechanical stimulation influences actin CSK structure and dynamics. The Monte Carlo technique could also be incorporated into network models to simulate active performances. Such as the microscopic dynamic model provided by Wang and Wolynes [108] in 2012 which is able to simulate active contractility that combines the motor-driven stochastic processes with the asymmetric load response of individual F-actin, or the finite element (FE) based discrete network model combined with stochastic crosslink scission kinetics [109] that can model the rate-sensitive stiffening-to-softening transition in F-actin networks induced by crosslink unbinding.

The dynamic behaviours of actin networks such as growth and 
symmetry-breaking also attracted adequate attention in modelling works. Those active behaviours are crucial in cellular activities. For instance, the growth of actin networks plays a critical role in the crawling mobility of almost all eukaryotic cells, and it is able to provide the force necessary for extending cell protrusions such as lamellipodia, as well as for propelling intracellular pathogens such as Listeria through the cytoplasm $[110,111]$. The individual F-actin in the networks could be modelled as dynamic 'ratchet' [27-29], which has been proposed to describe the effect of the filamentous polymerization. For example in 1999, van Oudenaarden and Theriot [112] developed a stochastic model for actin network, in which F-actins are modelled as elastic Brownian ratchets, which quantitatively accounts for the experimentally observed emergent symmetry-breaking behaviour. Several years later, the early researchers of the ratchet model further developed the ratchet model to a 'tethered ratchet' model [113], which explored the situation when the filaments are attached to the surface by assuming that the filaments attach to the surface transiently. In addition to the ratchet models, the dynamic behaviours of actin networks could also be achieved by other models. In 2001, to simulate the growth of branched actin networks against obstacles, Carlsson [110, 114] developed an 'Autocatalytic branching' model. It assumes that filaments branch off the sides or ends of existent filaments with the rate proportional to the number of the existent filaments. This model is capable of predicting whether the load force is dependent on the protrusion velocity. Then in 2004, Alberts and Odell [115] developed a 'nano-propulsion' model to study Listeria propulsion in realistic 3D geometry by numerically simulating every filament and all microscopic mechanical interactions in the comet-like actin tail. They modelled the molecular mechanics of the growth/disassembly of an actin network as it interacts with a moving rod-shaped bacterium. This in-silico reconstitution could produce persistent bacterial motion and actin tail morphology and explains how the observed 'runs-and-pauses' movements can emerge from a cooperative binding and breaking of attachments between F-actins and the bacterium. 
In 2012, a hybrid mesoscopic model for actin-based propulsion was developed by Zhu and Mogilner [116] to explain the observed bistability of the orientation of the ellipsoidal beads propelled by actin tails. The model incorporated both arrays of dynamic F-actins at the surface-tail interface and the bulk deformable actin gel behind the interface, which can explain both the concave-up and concave-down force-velocity relations for the growing actin networks depending on the characteristic time scale and network recoil.

The properties and behaviours of cell under the influence of the cell-generated forces have always been an intriguing topic in exploring cellular nature. For instance, such forces could drive changes in cell shape and Extra Cellular Matrix (ECM) remodelling, and contribute to the control of cell growth and function, as well as tissue patterning and mechanotransduction at the organ level [117, 118]. Specifically, the pre-stress existing in the actin networks could exert influence on cell, thus a significant development in the network modelling technique is to include the pre-stress. And the pre-existing stress is believed to be induced by activities like the contraction of cell components or cell distension on the substrate [119]. In 2003, Coughlin and Stamenović $[119,120]$ applied a prestressed cable network model [121, 122] on the actin network to model its deformability. The model is a network constituted of tensile cable elements (linear-elastic springs) without the balanced compression in the MTs. The model is able to obtain quantitative predictions of cell elastic properties. Another type of prestressed network model is the tensegrity model [121, 123], which was applied by Luo et al. [124] in 2008 to predict mechanical behaviours of actin networks in cell. The tensegrity model employs a discrete network of self-stabilizing pre-stressed tension bearing components which are balanced by locally compressed units, each subjected to mechanical equilibrium and geometric deformation. The mechanical behaviours that can be characterised by this model include viscoelastic retraction, fibre splaying after severing, non-uniform contraction, etc. 
The mechanotransduction involves the conversion of physical force into biochemical information, which is fundamental to development and physiology [125]. It provides a simple means by which cells and organisms can ensure structural stability, as well as a way to regulate morphogenetic movements to generate precise 3D structures [125]. And one of the versatile functions of actin networks is to play an important role in mechanotransduction in cell. Shafrir and Forgacs [126] constructed the network of F-actin with rigid rods connected with springs to study the mechanotransduction through the CSK. The model was submitted to either shock wave-like or periodic mechanical perturbations. The energy transfer properties of the actin network could be studied with this model. Later in 2012, Zeng et al. [127] developed a 3D random actin network model with randomly distributed linear Hookean springs. The model could be used to predict the deformation of the nucleus when mechanical stresses applied on the plasma membrane are propagated through the random cytoskeletal network to the nucleus membrane.

Besides the simulations mentioned above, there also have been numerous efforts to characterise mechanical responses of actin networks in models with different features. For instance in 2003, the network models of rigid rods mentioned earlier for mechanotransduction were utilized by Head et al. $[128,129]$ to study the regimes of elastic response and deformation modes of cross-linked networks. In the same year, the rigid network model was also utilized by Wilhelm and Frey [130] to characterise the scaling regimes of elasticity. The rigid rods model (Fig. 1.2(b)) could also be applied to the study of stress generation by myosin minifilaments in random actin network [105]. And recently, the effect of changes in F-actin length on cortex tension [22] was also identified by the rigid rods model. The similar type of models but with the filaments represented by elastic rods could also be applied on mechanical responses of actin networks. For instance in 2005, the transition from a bending-dominated response at small strains to a stretching-dominated response was explored by Onck et al. [131] using a 2D network model of filaments as elastic 
rods. In 2008, Roy and Qi [132] developed a micromechanical model to predict the average macroscopic elastic properties of the network. The model employs a unit cell consisting of four semiflexible chains and four equivalent axial-bending springs. The proposed unit-cell-based micromechanical model represents a statistically average realization of the actual network and gives the average mechanical properties, such as the shear modulus. In 2009 a kinematic model for describing turnover and reorientation of actin stress fibres (SFs) was developed by Kaunas and Hsu [133, 134]. The model is able to describe experimentally measured time courses of SF reorientation perpendicular to the direction of cyclic uniaxial stretch, as well as the lack of alignment in response to equibiaxial stretch. Four years later, a form finding model for F-actin network was proposed by Gong et al. [135] to explore the filament re-orientation under stretching. The analyses were carried out with large deformation nonlinear FE analysis with beam and cable elements used to model the F-actins and their cross-linkers. The model is able to predict experimental observations of filament re-orientation, and study the effects of the filament relative density, the filament length, and the relative density of filament cross-linkers on elastic modulus of the network. In 2014, a 2D network model of randomly oriented springs was applied to network mechanical responses [136]. It can link active self-organization of the actin network to the stiffness of the network and the traction forces generated by the network, and it is able to examine the response of adherent cells to stretch and changes in substrate stiffness [136]. In the same year, Li et al. [137] employed highly CG MD model developed earlier for actin network [138] to study the mechanisms of the compressive responses of F-actin networks. The model introduced the crosslinker unbinding mechanism dominated by deformation and is able to identify the dependency of network response on strain rate. Recently, a multiscale FEM model comprising the interaction of the actin network and cytosol was developed by Klinge et al. [139]. The model could be used to explore the filament orientation and its influence on the mechanical responses of network. 
The cell's ability to perform its function depends on its shape, which is maintained through structural stiffness and rheology. The role of actin network mechanics and rheology is important in accomplishing biological functions such as angiogenesis, wound repair, disinfection. To characterise the rheology of networks, researchers also proposed a wide range of computational models. For instance, in 2007, Kroy and Glaser [140, 141] introduced a Glassy WLC model, which is obtained from the common WLC by an exponential stretching of the relaxation spectrum of its long-wavelength eigenmodes. The model is able to give predictions such as the dynamic structure factor, and the microrheological susceptibility exhibit the characteristics of soft glassy rheology and compare favourably with experimental data for reconstituted cytoskeletal networks and live cells. Another example is the cross-link-governed dynamics model which used a combination of Monte Carlo simulations and an analytic approach [142]. The model could characterise long-time network relaxation controlled by cross-link dynamics by describing the structural relaxation that results from many independent unbinding or rebinding event. More detailed review of cytoskeletal network rheology could be found in [143], which brings together the primary experimental methods and theoretical and computational models regarding cytoskeletal rheology and mechanics.

It is worth mentioning that besides the categories of models introduced earlier, there also exist a variety of network models focused on particular issues which could be served as the references for the development of the future modelling techniques. For instance, the BD utilized in the modelling of single filament could also be applied on the investigation of morphology of actin network. The BD simulation model introduced by Kim et al. [144, 145] in 2009 could achieve the investigation of various actin-related phenomena such as analysing the effects of various system parameters on the growth and morphology of network and comparing the relative importance of unbinding and unfolding of actin cross-linking proteins in the dynamic properties of the network. Based on Kim's research, the later proposed 3D model 
with same foundation of BD [146] could simulate active cross-linked actin networks as systems that generate force as well as sense surrounding mechanical conditions. In 2013, an MD model for network restructuring was used by Alvarado et al. [147]. The model is able to examine the hypothesis that the stress-dependent binding kinetics allows motor activity to drive initially well-connected networks down towards a critically connected state. One year later, Alonso et al. [148] proposed a particle-based model for network based on the flocking theory, which could be understood as a distributed Kelvin-Voigt particle model. In this model, polymer chains are considered as point particles, while cross-linkers are represented as potential functions. This model can simulate a range of mechanical behaviours including strain hardening, viscoelastic creep, stress relaxation, network rupture, and network reformation. The viscoelastic Kelvin-Voigt element could also be coupled with the cross-bridge cycle of myosin motors to construct a Kelvin-Voigt-Myosin (KVM) model [149], which could be used to explain the dependence of cell fluidization on the stretching protocol, more specifically the contrast between the stretch-compress and compress-stretch manoeuvres. Later a thermodynamically consistent constitutive model was proposed by Fallqvist and Kroon [150] to explore the microstructure determined mechanical behaviour of networks. The model could predict many experimentally observed characteristics of networks, such as strain hardening, network rupture with subsequent softening and viscoelastic deformation.

\subsubsection{MT bundle}

Compared with the intensive focus on modelling the behaviours of actin networks, the investigations into MT/IF networks are relatively scarce. As mentioned earlier, MTs are essential components for cell. MT networks/bundles are subcellular structures of cross-linked MTs by MT-associated protein (MAP). And the network/bundle of MTs plays important roles in providing cell stiffness, intracellular organization, cell division, cell morphogenesis. Especially, a variety of neurological 
functions are mediated by the MT bundles, including maintaining mechanical integrity and shape of the axon, promoting axonal growth, and facilitating cargo transport $[8,151]$. Therefore, the modelling of them also attracted research attention. Buxton et al. [152] developed a stochastic model of MT network which describes the mechanics and kinetics of individual MTs, while at the same time presents the diffusion of tubulin and tau (proteins that stabilize MTs) concentrations. This model could include the effects of tau concentration and the hydrolysis of guanosine triphosphate-tubulin to guanosine diphosphate-tubulin and exhibit the emergence of MT dynamic instability. Another example is the bead-spring model (Fig. 1.2(c)) employed by Peter and Mofrad [106] to characterise the mechanical behaviour of axonal MT bundles in tension. The model could characterise the details of stiffening behaviour of MT bundle in tension and identify the primary deformation modes of bundle. Few years later, Allain and Kervrann [153] used particles to define a discretized connected MT network and simulated their mechanical dynamics using Newtonian mechanics. Euler Bernoulli theory was used to derive an elastic force that is applied at each node to account for the rigidity of MTs. The overall model enables linking cytosol to MTs dynamics in a constant state space thus allowing the use of data assimilation techniques.

\subsubsection{IF network}

The IF networks are subcellular structures of cross-linked IFs. The cell-specific IF network is often pictured as an integrator of F-actins and MTs via a complex set of cross bridging proteins [154]. Each specific IF network could integrate the cytoskeletal system of each cell into tissues and organs [154]. Ackbarow et al. [155] modelled a larger-scale network deformation of an IF model structure with a CG multi-scale model of alpha-helical protein domains using MD simulations. The model could relate the characteristic properties of networks to the particular nanomechanical properties of their protein constituents, and identify the role of 
proteins in protecting the network against catastrophic failure. Bertaud et al. [156] also utilized an empirical CG computational model of the IF network in eukaryotic cells. The study approximated the nonlinear force-extension behaviour of IFs under tension by a multilinear model and identified the significant role of IF in the cell mechanical behaviour at large deformation, and revealed the mechanistic insight into cell deformation under varying IF densities.

\subsubsection{MT with F-actin}

Researchers also attempted to model the MT linked with F-actin. The ability to sustain tension and compression offers the cell rigidity and maintains its structural stability [157]. It is generally admitted that F-actins could undergo tension while MTs are always compressed [157]. In 2011, Mehrbod and Mofrad [68] described a model of cross-linked MTs and F-actins (Fig. 1.2(d)). The overall configuration of the actin-MT connection resembles the deck-main cable connection in a suspension bridge. The model is able to simulate the semi-discrete loading pattern that mimics the filamentous environment around the MT filament instead of an elastic continuum environment [158].

\subsubsection{Summary}

Among the three major filaments of CSK, the modelling efforts are mainly placed on F-actin and MTs. The early ratchet model developed for F-actins concentrated on simulating the force generation during the cellular protrusion by actin polymerization and the early experiment applied the simple homogeneous assumption to model the structure of F-actin. Later in revealing the potential of various behaviours of F-actin that result from the particular 3D structure, models like NMA model and BD model were developed. And with the development of computational capability, MD/CGMD simulations were conducted to delineate the effect of detailed structures in subdomains of actin on structural and mechanical 
properties of F-actins. Moreover, efforts were also invested to improve computational efficiency for large scale F-actins in techniques like SSM model and MSM model.

Similar to the research focus on the force generation during the cellular protrusion by actin polymerization, the force-velocity relationship during the polymerization of MTs also attracted the attention of early researchers. Dynamical features such as length dependent growth rate and force could be predicted by such model. Investigation into other mechanical issues of MTs relies on the information of structure of MTs. The MTs had been treated as structures like shell, beam or lattice to characterise vibration and buckling behaviours. MD model could reveal the atomic information and give reasonable delineations in structural evolution or mechanics of MTs. The well-known WLC model exhibited its advantage in physics like thermal fluctuation of MTs. Moreover, the later developed atomistic-continuum model and MSM model could take more detailed features of MTs into consideration at lower computational cost.

Compared with the huge amount of models on MTs and F-actins, the models for IFs are relatively scarce. One example is the MD and CG models of IFs which could probe the response of the IF under mechanical tensile stretch.

Regarding the subcellular structures, the major concern of the modelling studies has been placed on the actin networks. The energy-based models were favoured by the early researchers. They could capture a variety of features of the actin networks as elastic properties, entanglement transition, motor-generated forces. The main focuses of a wide range of modelling approaches were placed on a limited number of topics like strain hardening and pre-stress. For instance, the strain hardening responses could be characterised by the open cell foam model, the updated-Lagrangian FEM model, elastic network model or WLC model. The dynamic behaviours of actin networks like growth and symmetry-breaking were simulated by the ratchet model, the 'Autocatalytic branching' model or the 
'nano-propulsion' model. In modelling the pre-stress, mechanotransduction and mechanical response, models like the tensegrity model, the rigid rods model and the form finding model were successfully applied. In addition, there are versatile models developed later, such as thermodynamically consistent constitutive model which could simulate a series of network behaviours. It is also worth mentioning that the networks rheology was characterised by models as the Glassy WLC model and the cross-link-governed dynamic.

Comparatively, the models for other subcellular structures are relatively scarce. However, those components like MT bundles/network are vital in biological processes of organisms. The few examples of those models in this topic are described in the present section. For example, the stochastic techniques, bead-spring model, particles model could be applied to mechanics and kinetics of MTs network/bundles. The IF network deformation was implemented with the CGMD model.

\subsection{Motivations of the research}

The field of CSK mechanics has undergone rapid development with great amount of effort into modelling of CSK filaments. In spite of the works achieved by the existing modelling studies, challenges still exist in the mechanical modelling of CSK filaments. More importantly, to facilitate the development of the CSK filament-based biosensor and biomimetic nanomaterials, it is crucial to further investigate the inherent (e.g., monomer interactions, helical structure) and environmental factors (e.g., surrounding proteins, cytosol, physical field) which can determine the nanomechanics of CSK filaments.

In theoretically characterising the mechanics of the CSK filaments, the models in the framework of continuum mechanics are unable to describe the monomeric features and the structural details of CSK filaments which may exert substantial influence on the overall mechanical behaviour of the protein polymer. Thus, MD technique and CG models were employed to study the monomeric features. However, 
with the introduced monomeric feature, largely reduced amount of calculation and improved computational efficiency are still needed to further explore the behaviours of large-scale filamentous structures, such as actin bundles and networks. Thus it is important to develop a cost-effective model for interpreting monomeric features of CSK filaments in a relatively larger scale (e.g., $\mu \mathrm{m}$ level).

Another fundamental issue in CSK mechanics is the relation between the CSK filaments structures and their mechanical behaviour and properties, namely, investigating the effect of unique helical structure on CSK filaments mechanics. This however has not been examined in detail until the development of a computational model of filaments with the feature of adjustable structural details. Fortunately, a recent study on the elastic properties of MTs based on a recently developed MSM model allows us to account for the structural details of CSK filaments at a low computational cost [77]. For example, the MSM simulation of the deformation of an MT composed of thousands of monomers only needs a personal PC with 4 cores to run less than one second, while the MD simulation of the deformation of a dimer composed of only two monomers needs a cluster with 56 cores to run more than one day. By utilizing, optimizing and upgrading the MSM techniques, it is expected that the finding of the effect of the unique helical structure of the filaments could be achieved.

In the current investigations about the CSK filaments, the individual filament itself can be well described and the consideration of surrounding liquid was also achieved in different studies. However, the filaments in CSK are not only in contact with the cytosol but also linked by other filaments. Thus, when studying the mechanics of individual CSK filaments, it is important to consider the influence of filamentous surrounding environment, which may lead to considerable changes in the mechanical behaviours. For instance, it was observed that MTs in vivo possess a critical buckling force higher than those in vitro, which could be attributed to the effect of the cross-linked status. 
An eternal motivation of fundamental research is to find a possible solution for converting the physical inspirations into a potential application. The mechanical features of CSK filaments could be used as biomarkers when possible changes of them are induced by physiological or pathological processes. Therefore, a coupled research of the interaction between the external physical field and the filaments is required, that could help us explore the possibility of utilizing CSK filaments as a non-invasive biosensor and provide a new avenue to a non-contacting technique.

\subsection{Thesis outline}

In this thesis, Chapter 1 reviewed the basic knowledge of CSK filaments and was devoted to summarise and classify a wealth of computational models for them, with concentrations on their functions and applications. The modelling techniques reviewed in the present thesis could deepen our understanding of the nature of CSK filaments and facilitate the development of novel diagnostics or biomimetic materials.

In Chapter 2, the basic concept for MSM models was introduced, followed by the modelling technique for MT-MSM model. Moreover, the validation of MT MSM model was also presented. Then an MSM model for F-actins in cells was developed, where the force constants describing the monomer interaction were achieved using MD simulations. After that, the mechanical properties measurements indicated that the obtained mechanical properties of the developed F-actin-MSM model were found to be in good agreement with existing experimental data.

Chapter 3 started to investigate the PF sliding and its effects on MT mechanics. It explored the structure-property relation for MTs and examined the relevance of the beam theories to their unique features. An MSM model was used to identify the origin of the inter-PF sliding and its role in bending and vibration of MTs. The beam models were then fitted to the MSM to reveal how they cope with the distinct mechanical responses induced by the inter-PF sliding. This study could reflect the 
distinct deformation mechanisms between an MT and its equivalent continuous body.

Chapter 4 studied helix structure effects on the mechanics of CSK filaments. A 3D transverse vibration was reported based on the MSM model for MTs, where the bending axis of the cross section rotates in an anticlockwise direction and the adjacent half-waves oscillate in different planes. A close correlation was confirmed between the rotation of the oscillation planes and the helical structures of MTs, showing that the 3D mode is a result of the helicity found in MTs. In addition, the tension-induced bending was studied for F-actins as a result of their helical structure.

Chapter 5 investigated the subcellular environment effect on the filament mechanics. The model of the cross-linkers system supporting MTs and the model of F-actin supported by the actin-binding proteins (ABPs) were developed based on the individual filament models introduced in Chapter 2. Then the effects of the cross-linkers on the buckling of MTs in cells and the effect of ABP on filopodial F-actin were examined. The study could demonstrate the important role of the cross-linker in regulating CSK filaments stiffness, reveal the physics of the experimentally observed buckling phenomena and pave the way to a new multi-component mechanics model for whole cells.

Chapter 6 explored electromechanical vibration of CSK filaments. The vibration of polarized MTs was excited by an alternating electric field (EF) and the EF-induced vibration modes as well as the associated frequency for MTs were captured. The frequency shift and stiffness alteration of MTs were also examined due to the possible changes of the tubulin interactions in physiological or pathological processes. Then the effect of nanoscale solid-liquid interface between MT and cytosol on the damping of electromechanical vibration of MTs in cytosol was explored. The strong correlation achieved between the tubulin interaction and MT vibration excited by $\mathrm{EF}$ could provide a new avenue to a non-contacting technique for the structural or property changes in MTs, where frequency shift is used as a biomarker. Meanwhile, the effect of nanoscale interface was found to reduce the 
damping and it may play an important role in designing MT-based biosensors and facilitating signal transduction in cells.

Chapter 7 summarised the contributions of the thesis and discussed the future research directions. 


\section{Chapter 2 Molecular structural mechanics models}

\subsection{Introduction}

The pivotal roles played by CSK depend crucially on the mechanical responses/properties of CSK filaments. For example, MTs are a structural element and primary organizer in the CSK of eukaryotic cells [49]. They form "tracks" on which motor proteins transport organelles and construct the spindle apparatus to facilitate cell division [50]. They are also responsible for maintaining the shape and providing the rigidity of the cells. F-actins are another main type of long filamentous polymers acting as basic structural components in eukaryotic cells [24, 25]. Owing to the unique molecular structures, F-actins have the ability to perform a broad range of essential cellular functions in cell motility as well as in locating and transporting protein complexes in cells [26].

The mechanics of MTs $[11,61,63,79,159-163]$ and F-actins[11-18, 164, 165] has been studied extensively in the last two decades, where the mechanical features of them are interpreted primarily via the CMMs. An in-depth understanding of CSK filaments mechanics is essential in revealing how cells fulfil their biological functions via CSK and offering new design of biomimetic structure/materials by mimicking the mechanical characteristics of CSK filaments. However, the CMMs models are unable to describe the structural details of CSK filaments which may exert substantial influence on the overall mechanical behaviour of the protein polymer [15]. Thus, MD technique and CG models were employed to study the monomeric features $[36,41,43,72,166]$. However, with the introduced monomeric feature, the largely reduced amount of calculation and the improved computational efficiency are still required in filament models to further explore the behaviours of large-scale filamentous structures, such as actin networks. Thus it is urgent to develop a cost-effective model for interpreting monomeric features of CSK filaments 
in a relatively larger scale (e.g., $\mu \mathrm{m}$ level). Compared with the dynamics simulations, the molecular structural mechanics (MSM) model is characterised by the static equilibrium feature. As a result, the MSM model is able to largely reduce the amount of calculation and improve computational efficiency, which is essential in the study of large-scale filamentous structures, such as actin bundles and MT CSK. Recently, the MSM modelling technique was applied in studies of the elastic properties of MTs [77]. In particular, the robustness and efficiency of the MSM model have been demonstrated in studying the mechanics of MTs [77, 78, 167]. And in the present chapter, the details of the MSM model for MT will be introduced.

Similar to MTs, F-actins are also a group of CSK components composed of individual filaments constructed by connecting protein monomers. The difference between MTs and F-actins lies in the overall configurations, i.e., an MT is a hollow cylinder formed by (say 13) filaments while an F-actin is a helical bundle made of two stranded protein filaments. Due to the efficient application of MSM model to MTs and the similarity between MTs and F-actin, it is of great interest to further extend the model to the mechanical behaviour of F-actin. Thus, in this chapter an MSM model for F-actins shall be developed where the force constants describing the monomer interaction are achieved using MD simulations.

\subsection{MSM model for MT}

\subsubsection{MT structure}

MTs are long tubular structures with neighbouring PFs shifted relatively to each other longitudinally, which results in helical structures as shown in Fig. 2.1 [168]. The basic components of MTs are $\alpha-\beta$ heterodimers [169] , which stack head to tail to form the PFs. MTs are finally constructed by a number of the PFs bonded laterally to form a cylindrical surface $[170,171]$. MTs have various architectures characterised by the PF number $N$ and the helix-start number $S$. For most MTs, $N=13$ and $S=3$ [172], but those with $N=8$ to 16 and $S=2$ to 4 are also reported in the 
literature. In particular, the latter lead to misfit in MTs and accordingly, generates a skew angle between the PF and axis of the MTs. [49, 54].

Fig. 2.1(a-c) showed the parameterized MTs structure characterised primarily by $N$ and $S$, which satisfy the equations below $[49,77,78,173]$ :

$$
\begin{aligned}
& r_{m}=\frac{S a_{m}}{N}-\delta x \tan \left(\theta_{m}\right) \\
& R=\frac{S a_{m} \tan \left(\theta_{m}\right)+N \delta x}{2 \pi}
\end{aligned}
$$

Herein $a_{m}$ is the subunit repeat, $r_{m}$ is the subunit rise, $R$ is the radius of the tube, $\delta x$ is the PF separation, and $\theta_{m}$ is the skew angle of PF relative to the direction of the rolling axis of MT. The values of PF separation, subunit repeat and skew angles of various MTs are present in Table 2.1 and considered in the simulations of the present study.

(a)

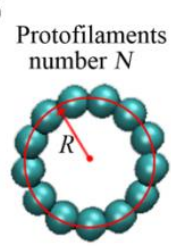

(b)

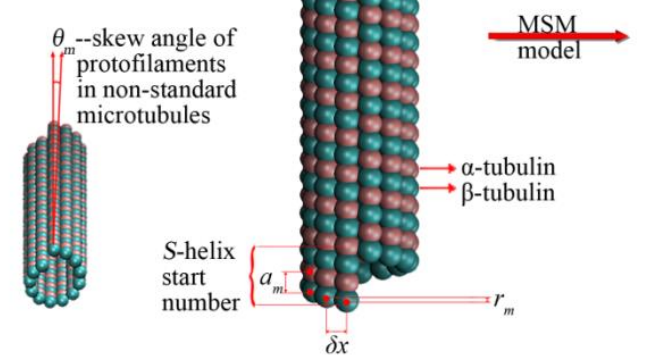

(d)

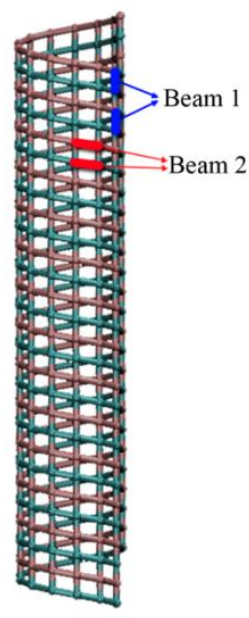

(e)

(f) Deformation Deformation $\begin{array}{ll}\text { of the tubulin } & \text { of the space } \\ \text { bonds in (c) } & \text { beams in (d) }\end{array}$
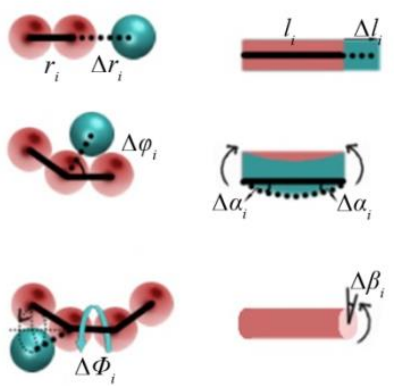

Figure 2.1 (a-c) Parameterized MT structure and (d) its MSM model; Here $a_{m}$ is the subunit repeat, $r_{m}$ is the subunit rise, $R$ is the radius of the MT, $\delta x$ is the PF separation and $\theta_{m}$ is the skew angle of PF relative to the direction of the axis of the MT. Vertical blue lines represent the intra-PF bonds of the MT and beam 1 of the MSM model, and the red ones denote the inter-PF bonds of the MT and beam 2 of the MSM model. (e) the deformation patterns of the tubulin bonds of the MT and (f) the space beams of the MSM model

\subsubsection{MSM model for MT}

To account for the structural details of MTs an MSM model [77] was developed for MTs. Similar techniques were also applied to the carbon nanotubes (CNTs) [174]. 
The MSM model was further employed in the present study due to its high computational efficiency and capability of accounting for the structural details of MTs. In particular, the MSM method was efficiently used to characterise the mechanical responses of MTs and found to be in good agreement with available experiments or atomistic simulations [77, 167].

Table 2.1 The structural details of various MTs [49]

\begin{tabular}{|c|c|c|c|c|c|c|c|c|c|c|c|c|}
\hline Parameter & \multicolumn{1}{|c|}{ Sym } & \multicolumn{10}{|c|}{ Value } \\
\hline $\begin{array}{c}\text { Proto- } \\
\text { filament } \\
\text { number }\end{array}$ & $N$ & 12 & 12 & 13 & 13 & 14 & 14 & 14 & 15 & 15 & 16 & $13^{*}$ \\
\hline $\begin{array}{c}\text { helix-start } \\
\text { number }\end{array}$ & $S$ & 2 & 3 & 2 & 3 & 2 & 3 & 4 & 3 & 4 & 4 & 0 \\
\hline $\begin{array}{c}\text { Proto- } \\
\text { Filament } \\
\text { separation }\end{array}$ & $\begin{array}{c}\delta x \\
(\mathrm{~nm})\end{array}$ & 5.18 & 5.27 & 5.02 & 5.13 & 5.08 & 5.16 & 5.05 & 5.07 & 5.06 & 5.19 & 5.13 \\
\hline $\begin{array}{c}\text { Subunit } \\
\text { repeat }\end{array}$ & $\begin{array}{c}a_{m} \\
(\mathrm{~nm})\end{array}$ & 4.05 & 4.04 & 4.07 & 4.05 & 4.06 & 4.05 & 4.05 & 4.05 & 4.04 & 4.04 & 4.05 \\
\hline $\begin{array}{c}\text { skew } \\
\text { angle }\end{array}$ & $\theta_{m}\left({ }^{\circ}\right)$ & -1.02 & 0.85 & -1.64 & 0 & -2.34 & -0.68 & 0.87 & -1.33 & 1.81 & 1.17 & 0 \\
\hline
\end{tabular}

* Imaginary (non-helical) MT

The space structure of MTs is shown in Fig. 2.1, where the intra-PF $\alpha \beta$ interactions, i.e., bond1 (represented by the blue lines in Fig. 2.1(c)), are modelled as the elastic space beam 1 and the inter-PF $\alpha \alpha(\beta \beta)$ interaction, i.e., bond 2 (denoted by the red lines in Fig. 2.1(c)), are treated as the elastic space beam 2. Following previous studies $[77,78,175]$, the small difference in $\alpha \alpha$ and $\beta \beta$ interactions is neglected. Such a frame structure consisting of the space beams is then obtained as an MSM model for an MT.

In molecular mechanics, the force field is expressed in the form of steric potential energy. The major parts of the steric potential energy of an MT structure include the bond stretching energy $U_{i}^{r}$, the angle bending energy $U_{i}^{\varphi}$ and the dihedral angle torsional potential energy $U_{i}^{\tau}$. The total potential energy $U$ of an MT reads 


$$
U_{\text {bonds }}=\sum\left(\sum U_{i}^{r}+\sum U_{i}^{\varphi}+\sum U_{i}^{\tau}\right)
$$

where $i$ denotes the types of bonds mentioned above ( $i=1$ for the intra-PF bonds and $i=2$ for the inter-PF bonds). The expressions for the three types of bond energy are as follows.

$$
U_{i}^{r}=\frac{1}{2} k_{i}^{r}\left(\Delta r_{i}\right)^{2}, U_{i}^{\varphi}=\frac{1}{2} k_{i}^{\varphi}\left(\Delta \varphi_{i}\right)^{2}, U_{i}^{\tau}=\frac{1}{2} k_{i}^{\tau}\left(\Delta \Phi_{i}\right)^{2},(i=1,2)
$$

Here, $\Delta r_{i}$ is the change of bond length, $\Delta \varphi_{i}$ is the change of in-plane bond angle, $\Delta \Phi_{i}$ is the change of out-of-plane angle, $k_{i}^{r}$ is the force constant for bond stretching, $k_{i}^{\varphi}$ is the force constant for bond angle bending and $k_{i}^{\tau}$ is the force constant for bond torsion. The values of these force constants can be obtained in atomistic simulations or experiments.

In addition, the total potential energy of the MSM model can be written as:

$$
U_{\text {beams }}=\sum_{i=1,2}\left(\sum U_{i}^{A}+\sum U_{i}^{M}+\sum U_{i}^{T}\right)
$$

where, $U_{i}^{A}$ is the strain energies of a beam in tension. $U_{i}^{M}$ is the strain energy due to bending and $U_{i}^{T}$ is the strain energy due to torsion. Here $i$ specifies the quantities of beam $i$ ( $i=1$ for longitudinal beams and $i=2$ for lateral beams). The beam energy can be calculated by using the formulae below.

$$
U_{i}^{A}=\frac{1}{2} \frac{Y_{i} A_{i}}{l_{i}}\left(\Delta l_{i}\right)^{2}, U_{i}^{M}=\frac{1}{2} \frac{Y_{i} I_{i}}{l_{i}}\left(2 \Delta \alpha_{i}\right)^{2}, U_{i}^{T}=\frac{1}{2} \frac{S_{i} J_{i}}{l_{i}}\left(\Delta \beta_{i}\right)^{2},(i=1,2)
$$

Here, $\Delta l_{i}$ is the length change of the beam, $\Delta \alpha_{i}$ is the bending angle, $\Delta \beta_{i}$ is the torsion angle, $Y_{i} A_{i}$ is the extensional stiffness, $Y_{i} I_{i}$ is the bending stiffness $S_{i} J_{i}$ is the torsional stiffness of the beam.

The equivalency of the MT structure and its MSM model can be established when the corresponding energy in Eqs. 2.3 and 2.5 are equal, which leads to the following relationship between the force constants of the bonds and the stiffnesses of the space beams.

$$
\frac{Y_{i} A_{i}}{l_{i}}=k_{i}^{r}, \frac{Y_{i} I_{i}}{l_{i}}=k_{i}^{\varphi}, \frac{S_{i} J_{i}}{l_{i}}=k_{i}^{\tau},(i=1,2)
$$


In the MSM model the values of $k_{i}^{r}, k_{i}^{\varphi}, k_{i}^{\tau}$ were obtained from the MD simulations $[70,77, \quad 167]$, i.e., $k_{l}^{r}=3 \mathrm{nN} / \mathrm{nm}, \quad k_{I}^{\varphi}=2 \mathrm{nN} \cdot \mathrm{nm}, \quad k_{l}^{\tau}=0.04 \mathrm{nN} \cdot \mathrm{nm}$, $k_{2}^{r}=14 \mathrm{nN} / \mathrm{nm}, k_{2}^{\varphi}=8.5 \mathrm{nN} \cdot \mathrm{nm}, k_{2}^{\tau}=0.17 \mathrm{nN} \cdot \mathrm{nm}$. The mass of monomer was taken as $M_{m o n o}=55 \mathrm{kDa}[78,176]$.

Subsequently, the stiffness matrices $\mathbf{K}$ can be constructed for the whole frame structure of MT (see details in Sec. 2.2.3) and its static deformation can be calculated by using the stiffness matrix method based on the following equation

$$
\mathbf{K u}=\mathbf{F}
$$

where $\mathbf{u}$ is the global nodal displacements and $\mathbf{F}$ is the nodal forces acting on the boundary of an MT.

\subsubsection{The mass matrix and stiffness matrix for MSM model}

In the present MSM model, the mass for beam element was calculated as $M_{t}=\rho_{b} A_{b} l_{b}$, where $\rho_{b}$ is the density of the beam element $A_{b}$ is the area of the beam and $l_{b}$ is the length of the beam.

Then the element mass matrix $\mathbf{M}^{\mathbf{e}}$ is calculated as per FE method and is given by:

$$
\mathbf{M}^{\mathbf{e}}=\frac{M_{t}}{2}\left[\begin{array}{cccccccccccc}
1 & 0 & 0 & 0 & 0 & 0 & 0 & 0 & 0 & 0 & 0 & 0 \\
0 & 1 & 0 & 0 & 0 & 0 & 0 & 0 & 0 & 0 & 0 & 0 \\
0 & 0 & 1 & 0 & 0 & 0 & 0 & 0 & 0 & 0 & 0 & 0 \\
0 & 0 & 0 & 0 & 0 & 0 & 0 & 0 & 0 & 0 & 0 & 0 \\
0 & 0 & 0 & 0 & 0 & 0 & 0 & 0 & 0 & 0 & 0 & 0 \\
0 & 0 & 0 & 0 & 0 & 0 & 0 & 0 & 0 & 0 & 0 & 0 \\
0 & 0 & 0 & 0 & 0 & 0 & 1 & 0 & 0 & 0 & 0 & 0 \\
0 & 0 & 0 & 0 & 0 & 0 & 0 & 1 & 0 & 0 & 0 & 0 \\
0 & 0 & 0 & 0 & 0 & 0 & 0 & 0 & 1 & 0 & 0 & 0 \\
0 & 0 & 0 & 0 & 0 & 0 & 0 & 0 & 0 & 0 & 0 & 0 \\
0 & 0 & 0 & 0 & 0 & 0 & 0 & 0 & 0 & 0 & 0 & 0 \\
0 & 0 & 0 & 0 & 0 & 0 & 0 & 0 & 0 & 0 & 0 & 0
\end{array}\right]
$$

The element elastic stiffness matrix $\mathbf{K}^{\mathbf{e}}$ of the MSM model is calculated as:

$$
\mathbf{K}^{\mathbf{e}}=\left[\begin{array}{cc}
K_{a a} & K_{a b} \\
K_{a b}^{T} & K_{b b}
\end{array}\right]
$$

where the sub-matrices are obtained via the following equations and the beam 
stiffnesses $Y A, Y I, S J$ given by Eq. 2.7.

$$
\begin{gathered}
\boldsymbol{K}_{\boldsymbol{a} \boldsymbol{a}}=\left[\begin{array}{cccccc}
Y A / l & 0 & 0 & 0 & 0 & 0 \\
0 & 12 Y I / l^{3} & 0 & 0 & 0 & 6 Y I / l^{2} \\
0 & 0 & 12 Y I / l^{3} & 0 & -6 Y I / l^{2} & 0 \\
0 & 0 & 0 & S J / l & 0 & 0 \\
0 & 0 & -6 Y I / l^{2} & 0 & 4 Y I / l & 0 \\
0 & 6 Y I / l^{2} & 0 & 0 & 0 & 4 Y I / l
\end{array}\right] \\
\boldsymbol{K}_{\boldsymbol{a} \boldsymbol{b}}=\left[\begin{array}{cccccc}
-Y A / l & 0 & 0 & 0 & 0 & 0 \\
0 & -12 Y I / l^{3} & 0 & 0 & 0 & 6 Y I / l^{2} \\
0 & 0 & -12 Y I / l^{3} & 0 & -6 Y I / l^{2} & 0 \\
0 & 0 & 0 & -S J / l & 0 & 0 \\
0 & 0 & 6 Y I / l^{2} & 0 & 2 Y I / l & 0 \\
0 & -6 Y I / l^{2} & 0 & 0 & 0 & 2 Y I / l
\end{array}\right] \\
\boldsymbol{K}_{\boldsymbol{b} \boldsymbol{b}}=\left[\begin{array}{cccccc}
Y A / l & 0 & 0 & 0 & 0 & 0 \\
0 & 12 Y I / l^{3} & 0 & 0 & 0 & -6 Y I / l^{2} \\
0 & 0 & 12 Y I / l^{3} & 0 & 6 Y I / l^{2} & 0 \\
0 & 0 & 0 & S J / l & 0 & 0 \\
0 & 0 & 6 Y I / l^{2} & 0 & 4 Y I / l & 0 \\
0 & -6 Y I / l^{2} & 0 & 0 & 0 & 4 Y I / l
\end{array}\right]
\end{gathered}
$$

The assembling procedure from the elemental matrices $\left(\mathbf{M}^{\mathbf{e}}, \mathbf{K}^{\mathbf{e}}\right)$ to the structural mass matrix $\mathbf{M}$ and the structural stiffness matrix $\mathbf{K}$ follows the node-related technique in the FE method [177].

\subsubsection{Validation of MT MSM model}

In this section, the validation of MSM model for MT will be introduced [77]. The MSM simulations were carried out with a desktop computer with 4 cores. The MSM model for MT was validated by measuring the mechanical properties of MTs, and by comparing the obtained results with the experimental data [77]. The free-fixed end conditions (i.e., one end is free and the other is fixed) were considered for the sample MTs unless otherwise specified [77]. The Young's modulus of the sample MTs was measured firstly in tensile test, where the free end of the MTs was subject to an axial force [77]. The Young's modulus rises and falls around a mean value $0.83 \mathrm{GPa}$ with the maximum value around $0.85 \mathrm{GPa}$ and the minimum value 
around $0.8 \mathrm{GPa}$ [77]. It is found that the mean value of Young's modulus $0.83 \mathrm{GPa}$ predicted based on the MSM model for different MTs agrees well with $0.8 \mathrm{GPa}$ obtained in an experiment [178] (the relative difference $4 \%$ ) and $0.9 \mathrm{GPa}$ calculated for 13-3 MTs based on the AENM [72] (the relative difference around $8 \%$ ) [77].

Then the shear modulus of MTs was measured based on the MSM model [77]. In this test, a torsional moment was applied to the free end of an MT and the generated torsional angle was recorded to compute the shear modulus [77]. Here, 47.1MPa was achieved for 13-3 MTs based on the MSM model, which is more than two orders of magnitude lower than the Young's modulus $0.83 \mathrm{GPa}$ [77]. The result agrees well with 47MPa [179] and 50MPa [72] reported in other studies [77].

Finally, the MSM model was utilized to measure the effective bending stiffness of MTs [77]. In this case, a transverse force was applied to the free end of the MT to generate the deflection at the same place [77]. The bending stiffness of such a cantilever beam can then be evaluated. The obtained bending stiffness fall in the range of $\left[2.2 \times 10^{-24} \mathrm{~N} \mathrm{~m}^{2}, 20.2 \times 10^{-24} \mathrm{~N} \mathrm{~m}^{2}\right]$ [77], which is in perfect agreement with $\left[1.9 \times 10^{-24} \mathrm{~N} \mathrm{~m}^{2}, 21.5 \times 10^{-24} \mathrm{~N} \mathrm{~m}^{2}\right]$ reported in experiments $[11,162,180]$.

\subsection{MSM model for F-actin}

\subsubsection{The structure of $\mathbf{F}$-actin}

F-actins are long filamentous polymers acting as basic structural components in eukaryotic cells $[24,25]$. The filamentous structure is established via an assembly process from monomeric globular $(\mathrm{G})$ actin subunits to fibrous (F) actin [181]. The twist of the actin helix determines how the subunits are positioned with respect to each other [182]. Though there exist various twists, the most frequently reported is the twin strand of beads with an angular separation between the subunits in the helix $[182,183]$ 


\subsubsection{MSM model for F-actin}

The structure of a single F-actin was illustrated in Fig. 2.2(a) where the building blocks are G-actin monomers. X-ray studies revealed that, in most cases the angular separation $\theta_{a}$ in F-actins varies from 167.14 to $166.15^{\circ}$ [182]. Accordingly, the two typical structures of F-actin were considered in the present study, which were associated with the angular separation $\theta_{a}=167.14^{\circ}$ and $166.15^{\circ}$, respectively. The

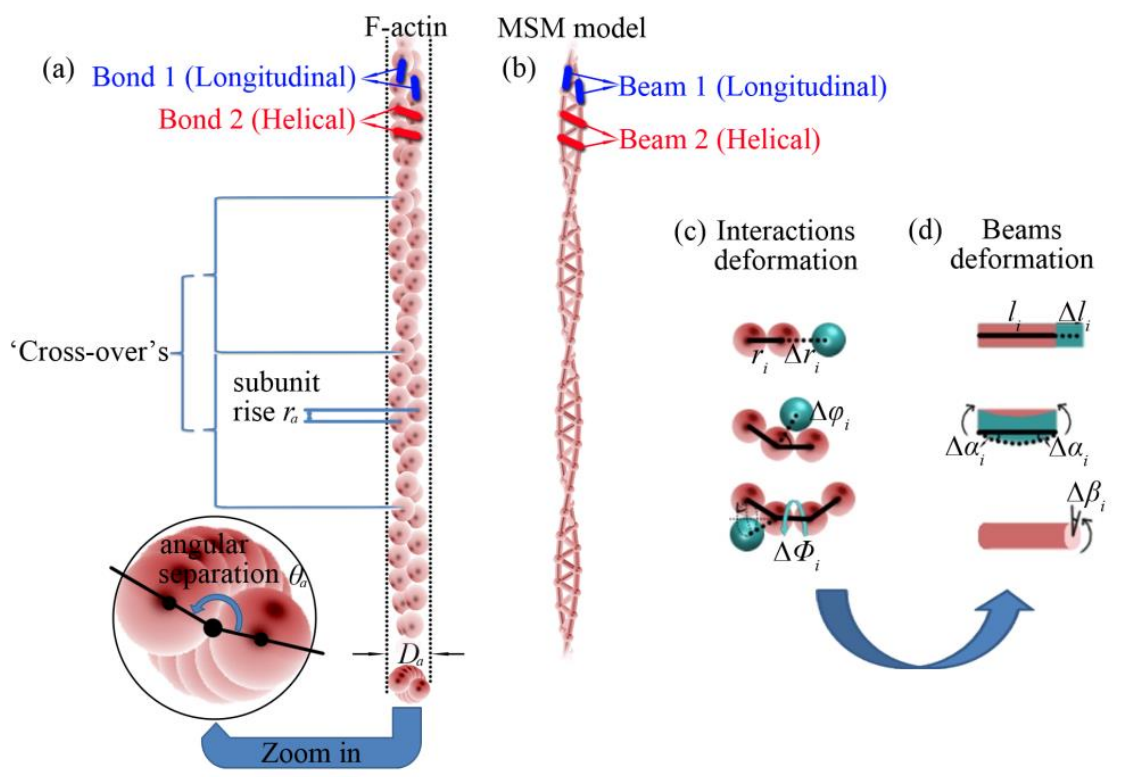

Figure 2.2 (a) Structural representation of an F-actin with monomer interactions 1 and 2, (b) the MSM model developed for the F-actin with elastic beams 1 and 2 characterising interactions 1 and 2, respectively, the deformation patterns of (c) the monomer interactions of F-actin and (d) the elastic beams 1 and 2 of the MSM model.

difference between these two typical cases thus showed the maximum influence of the variation of angle separation on the mechanical behaviour of F-actin. The subunit rise of F-actin $r_{a}$ was reported as $2.73 \mathrm{~nm}$ [182]. Different diameters $D_{a}$ of F-actin were reported in the literature. In this study, $D_{a}=7 \mathrm{~nm}$ was selected [184], which yields the area of cross-section around $19 \mathrm{~nm}^{2}$ close to $18 \mathrm{~nm}^{2}$ previously used [11] in calculating the mechanical properties of F-actin. In the MSM model (Fig. 2.2(b)) of the present study, the cross-section was modelled as two tangent circles as the resultant helical geometry is a helix consisting of two strands of head-to-tail stacked monomers [26]. Moreover, the helical repeat of F-actin is denoted as 'cross-over' 
where the number of subunits varies with the angular separation $\theta_{a}$ [182].

The equivalency of the F-actin structure and its MSM model (Fig. 2.2(b)) can be established with the same methods as indicated in Sec. 2.2.2 for MT MSM model (Fig. 2.2(c-d)), which also leads to the following relationship between the force constants of the actin interactions and the stiffnesses of the space beams.

$$
\frac{Y_{i} A_{i}}{l_{i}}=k_{i}^{r}, \frac{Y_{i} I_{i}}{l_{i}}=k_{i}^{\varphi}, \frac{S_{i} J_{i}}{l_{i}}=k_{i}^{\tau},(i=1,2)
$$

Thus, the structural stiffnesses can be obtained via Eq. 2.13 once the values of $k_{i}^{r}, k_{i}^{\varphi}, k_{i}^{\tau}$ were obtained for F-actin based on MD simulations. The mass of monomer was taken as $M_{m o n o}=42 \mathrm{kDa}[185]$.

\subsubsection{MD simulations on protein interaction}

In this section, we obtained the force constants for the protein interactions of F-actin based on MD simulations. After that, the structural stiffnesses of the space beams required in the MSM model can be determined based on Eq. 2.13. The MD simulations and the experimental setup used are introduced briefly in this section. As shown above, F-actins are helical linear polymers composed of G-actin subunits [182]. Following the previously used numbering method, each subunit is labelled by an integer, $n-1, n, n+1$ or $n+2$ (Fig. 2.3(a)) from the barbed-end side of the filament [26]. The intra-strand interaction (interaction 1) of G-actins was defined as longitudinal interaction and the inter-strand one (interaction 2) as the helical interaction. Both intra- and inter-strand interactions comprise of two different interactions, such as the interaction between two monomers at their interface and the interaction inside individual monomers.

To characterise these interactions, an AFM-like MD approach was applied [186]. The atomic structure of monomeric actin labelled by PDB ID code 1J6Z [187] was considered in the development of the model. The details of the structure can be found in the RSCB Protein Data Bank [188]. Herein, two different molecular systems 
shown in Fig. 2.3(b-e) were generated, i.e., the intra-strand $(n \sim n+2)$ interaction and

(a)

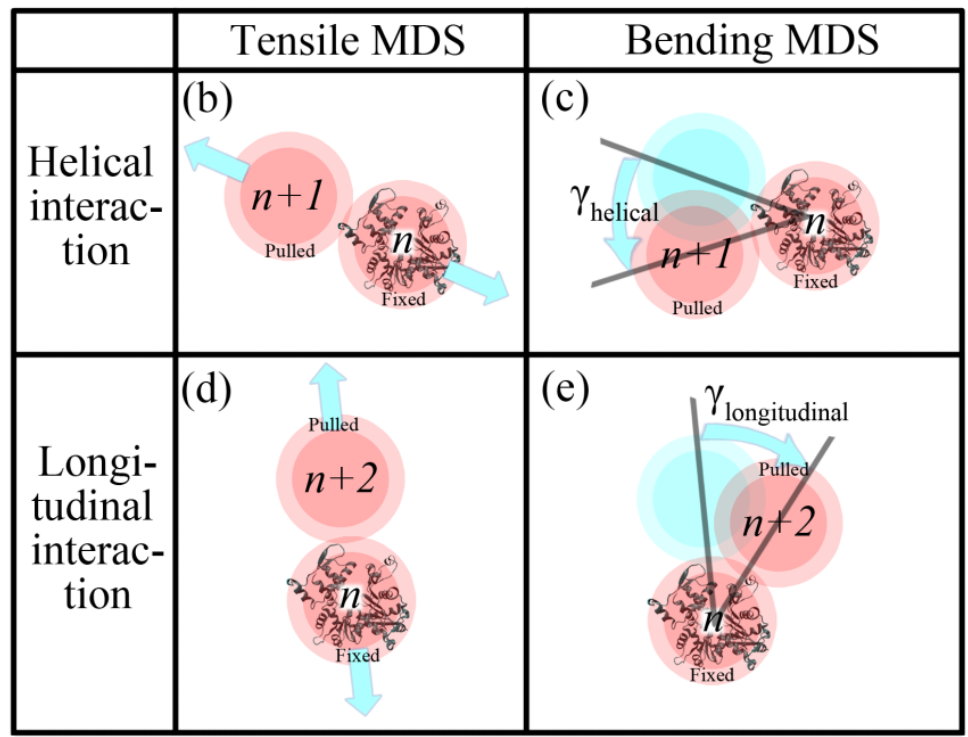

Figure 2.3 Schematics of an F-actin structure showing (a) numbering method of the monomers and the deformation patterns of the monomer interactions including (b) the interaction stretching and (c) interaction bending in the helical direction, and (d) the interaction stretching and (e) interaction bending in the longitudinal direction.

the inter-strand $(n \sim n+1)$ interaction, each of which consists of two monomers. The filament model by Egelman et al. [182] was employed to describe the initial organization between monomers. The structural data of globular (G-) actin are used in the present MD simulations to represent those of F-actin with conformation or filamentous state different from that of G-actin [187]. Such a replacement was applied successfully to characterise F-actin in previous studies [36, 41]. The characterisation of the inter-strand monomer interaction (between the monomers $n$ and $n+1$ (Fig. 2.3(b-c))) was performed via MD simulations where a group of $\mathrm{C}_{\alpha}$-atoms of the residues in monomer $n+1$ (denoted as Pulled-G) were pulled by an external load (Fig. 2.3(b-c)), while another group of $\mathrm{C}_{\alpha}$-atoms of the residues from monomer $n$ (denoted as Fixed-G) were fixed. Similar techniques were used for the intra-strand monomer interaction (between monomers $n$ and $n+2$ (Fig. 2.3(d-e))). In this case, the Pulled-G and Fixed-G were chosen from monomer $n+2$ and monomer $n$, respectively. Herein, the Pulled-G and Fixed-G were chosen from the residues involved in the interactions between the actin monomers in F-actin structure by 
following Ref. [26]. In addition, more detailed study was conducted to examine the influence of different choices [26] of the groups. It was found that the force-displacement relation only changes slightly when different groups were selected. Thus, the results based on the selected Pulled-G and Fixed-G in the present work should be reliable. The entire system was solved by water molecules, neutralized with Potassium chloride [41], and then energy minimized, heated to $310 \mathrm{~K}$ and equilibrated for $5000 \mathrm{ps}$ in order to stabilize the structure [189]. Then the Pulled-G were pulled by connected springs with a preliminary assigned elastic constant $k_{e s}=6.948 \mathrm{nN} / \mathrm{nm}$. The selected value of $k_{e s}$ represents a compromise between low $k_{e s}$ values that make the MD simulations very time consuming and high $k_{e s}$ values which allow faster simulations but introduce large uncertainty in the force values. The Langevin dynamics was specified as inactive during the steered MD (SMD) simulations in order to disturb the movement of the atoms as little as possible. In the tensile and bending tests, the free end of a spring was moved at a constant rate [69]. It is noted that the force-deformation behaviour of proteins (e.g., collagen triple helix) may show the strain rate-dependence in tensile test [190]. Thus, in this study we examined the influence of strain rate on the force constants. It was found that the equivalent force constant converges when the rate is under $1 \times 10^{-4} \mathrm{~nm} / \mathrm{ps}$. Hence, in what follows the rate $5 \times 10^{-5} \mathrm{~nm} / \mathrm{ps}$ was used in the simulations. Three replicas of simulations were then performed for each interaction system. The values of material property were calculated as the average value of the material property achieved in the three simulations.

More technical details of the MD simulation are presented in the following paragraph.

The MD simulations were carried out in a cluster computer with 56 cores. The MD simulations were performed based on NAMD package (a parallel molecular dynamics code designed for high-performance simulation of large biomolecular systems) [186]. The NAMD package has been widely used to make breakthroughs in 
understanding the structure and dynamics of various large biomolecular complexes [191]. The CHARMM22 force field was employed in the simulations which could provide a consistent set for condensed-phase simulations of a wide variety of molecules of biological interest [192]. The CHARMM22 force field is widely used in the protein simulations with water involved and is proven to be accurate in predicting SMD results [193]. The cut-offs used for the non-bond interactions (van der Waals and electrostatic) were set to $1.2 \mathrm{~nm}$ which was reported as the convergence for the cut-offs of non-bond interactions [194, 195]. Smoothing functions are applied to both the electrostatics and vdW forces. The time step was set to $2 \mathrm{fs}$ for all the MD simulations. The structure was centred in a rectangular box of size $12.3 \times 8.2 \times 10 \mathrm{~nm}$ with periodic boundary conditions imposed. The rest of the box was filled with about 25000 transferable intermolecular potential 3P(TIP3P) [196] water molecules to model water in the system. To balance the charge and neutralize the whole system with ions, Potassium chloride was added to the solution [41]. In the box, there were about 88000 atoms in the system. The entire system was heated to $310 \mathrm{~K}$ and the temperature was controlled by Langevin Dynamics. Meanwhile, Particle Mesh Ewald (PME) method was introduced to deal with electrostatic interactions in the system with periodic boundary conditions. Then the system was energy minimized for $20 \mathrm{ps}$ and left to equilibrate for $5000 \mathrm{ps}$ in order to stabilize the structure in terms of temperature and energy oscillations. The equilibrated structure was the basis for all further dynamics done for the mechanical characterisation. The mechanical properties of the equilibrated systems were tested using the SMD simulation. It should be noted that the systems were still solvated by water and neutralized, and the number of atoms was also about 88000 . Two groups of $\mathrm{C}_{\alpha}$-atoms were chosen as the fixed group of atoms and the pulled group of atoms. The equilibrated system was energy minimized again for 20 ps before the pulling procedure. Then the constant velocity pulling method was applied and the pulled group of atoms were pulled by connected springs with a preliminary assigned elastic constant $k_{e s}=6.948 \mathrm{nN} / \mathrm{nm}$. 
The pulling direction was given as the normalized direction between the pulled atoms and the fixed atoms. To determine the pulling velocity one should consider the balance between the accuracy and the computer power available. Therefore, the tensile simulations were performed on the inter-strand system with different velocities (from $2.5 \times 10^{-5} \mathrm{~nm} / \mathrm{ps}$ to $5 \times 10^{-3} \mathrm{~nm} / \mathrm{ps}$ ) to find out the appropriate velocity (convergence) to do the simulations on the rest of the systems. When determining the range of tested velocity, a reference velocity of $5 \times 10^{-4} \mathrm{~nm} / \mathrm{ps}$ [69] from the pulling simulation of a similar system was taken into consideration. Finally, the velocity of $5 \times 10^{-5} \mathrm{~nm} / \mathrm{ps}$ was used in the simulations of other systems and 3 replicas of simulations were performed for each interaction system. From the generated data of SMD simulation, the trajectory of the atoms and the force applied to the pulled group of atoms could be extracted. In order to obtain the force in the direction of pulling, the dot product of the force and the normalized direction of pulling was calculated. Finally, the force-displacement figure could be plotted based on those data.

The results from the AFM-like MD simulations were shown in Fig. 2.4 describing the relation between the load on the Pulled-G and their displacement along the pulling direction. Following previous MD simulations [69], the maximum displacement $0.5 \mathrm{~nm}$ or $1 \mathrm{~nm}$ is considered for the tensile and bending tests of the monomer interactions. This displacement range is selected as it reflects the range of displacement of subcellular components observed experimentally in Refs. [11, 13, 15]. From linear fitting to the data in Fig. 2.4(a-b), the force constants of actin stretching were obtained, i.e., $k_{\text {helical }}^{\text {stretching }}=0.88953 \mathrm{nN} / \mathrm{nm}$ for helical interactions and $k_{\text {longitudinal }}^{\text {stretching }}=3.13099 \mathrm{nN} / \mathrm{nm}$ for longitudinal interactions. It is worth mentioning that the linear interaction assumption is adopted to facilitate the development of the MSM for constituent protein monomers of F-actin. Such an assumption was efficiently used previously in developing models for and understanding the experimentally observed mechanical behaviour of protein filaments $[11,69,70,77,161,162]$. The assumption is necessary here as it can 
greatly improve the computational efficiency of the MSM and at the same time, maintain the accuracy in solving the mechanical problems with relatively small deformation. On the other hand, in future efforts should be invested to improving the MSM model by considering the non-linear monomeric interaction and enabling the use of the MSM in large deformation problems of F-actin.

In the simulation of bending, the distance between the Pulled-G and Fixed-G was obtained as $6.046 \mathrm{~nm}$ and $8.644 \mathrm{~nm}$ in the helical and the longitudinal directions, respectively. When the translational displacement of Pulled-G is $1.0 \mathrm{~nm}$ in bending, the interaction angle changes $\gamma_{\text {helical }}=0.16559 \mathrm{rad}$ and $\gamma_{\text {longitudinal }}=0.11575 \mathrm{rad}$ were
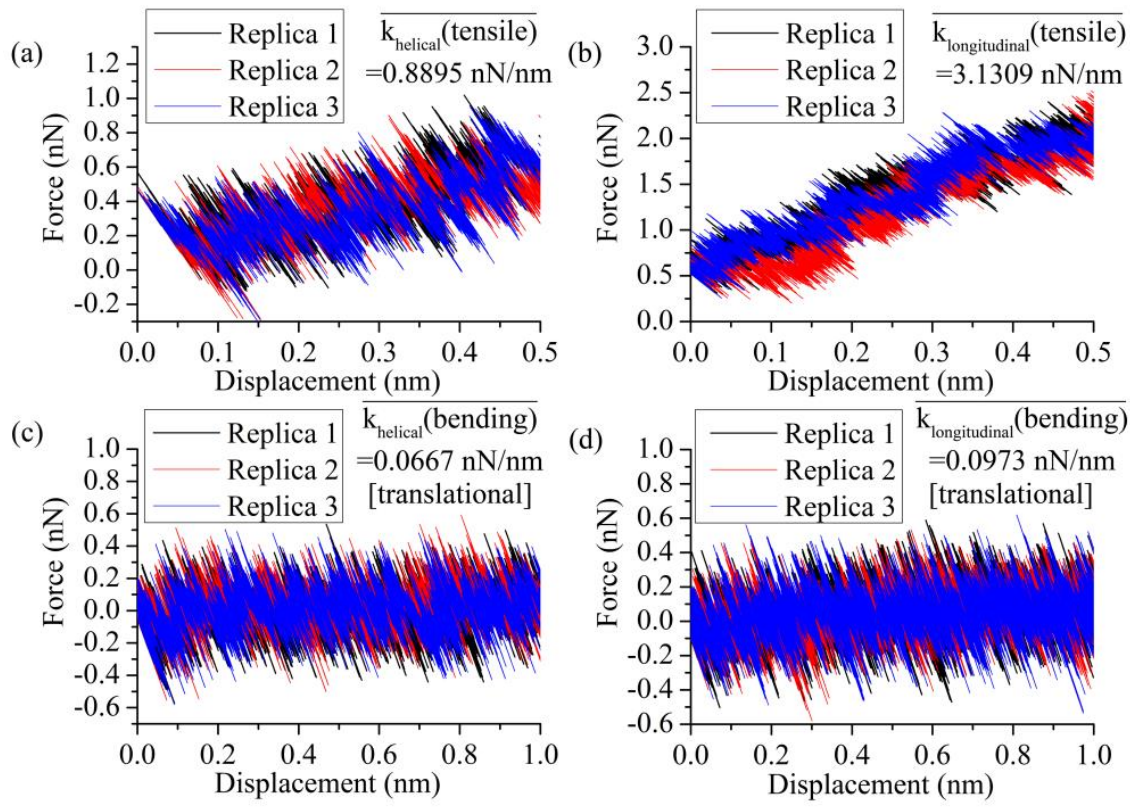

Figure 2.4 Force-displacement relation obtained for Pulled-G in pulling direction for interaction stretching along (a) the helical and (b) the longitudinal directions, and interaction bending in (c) the helical and (d) the longitudinal directions.

obtained, respectively. Herein, the bending energy is calculated by $U=\frac{1}{2} k_{\text {translational }}^{\text {bending }}\left(\Delta l_{p}\right)^{2}$ or $U=\frac{1}{2} k_{\text {angular }}^{\text {bending }}(\gamma)^{2}$, where $k_{\text {translational }}^{\text {bending }}$ is the interaction constant that can be directly obtained from Fig. 2.4(c-d), $\Delta l_{p}$ is the displacement of Pulled-G in pulling direction, $\gamma$ is the change of interaction angle and $k_{\text {angular }}^{\text {bending }}$ is defined as the force constant for the bending. Based on information shown above we finally arrived at $k_{\text {angular }}^{\text {bending }}=k_{\text {translational }}^{\text {bending }} \frac{\left(\Delta l_{p}\right)^{2}}{(\gamma)^{2}}$ and $k_{\text {helical }}^{\text {bending }}=$ 
$2.43112 \mathrm{nN} / \mathrm{nm}$ for the helical interactions and $k_{\text {longitudinal }}^{\text {bending }}=7.26357 \mathrm{nN} / \mathrm{nm}$ for longitudinal interactions. It is noticed that the torsion of G-actins is difficult to simulate with the AFM-like MD simulation method. Thus, following the empirical treatment in Ref. [70], the force constants for the torsion of the two interactions were set as $k_{\text {helical }}^{\text {torsion }}=k_{\text {helical }}^{\text {bending }} / 50$ and $k_{\text {longitudinal }}^{\text {torsion }}=k_{\text {longitudinal }}^{\text {bending }} / 50$. The force constants were summarised in Table 2.2, which are obtained for the interactions between two adjacent G-actins. The stiffness of a system of four G-actins was also evaluated in Ref. [138]. Using the MSM technique, the equivalent stiffness of the four G-actin systems can then be estimated based on the force constants obtain for the two actin systems in the present simulations. The result $6.67 \mathrm{nN} / \mathrm{nm}$ is found to be within the range of $[4.16,67.7 \mathrm{nN} / \mathrm{nm}]$ achieved in the previous MD simulations [138]. Also, as will be shown later, the mechanical properties of F-actin given by the MSM model based on the obtained force constants match with many existing experimental data [11-18, 182, 184, 197-217].

Table 2.2 The force constants of interactions between monomers of F-actin

\begin{tabular}{|c|c|c|}
\hline Description & Constant symbol & Value \\
\hline Longitudinal tensile & $k_{\text {longitudinal }}^{\text {stretching }}\left(k_{1}^{r}\right)$ & $3.13 \mathrm{nN} / \mathrm{nm}$ \\
\hline Longitudinal bending & $k_{\text {longitudinal }}^{\text {bending }}\left(k_{1}^{\varphi}\right)$ & $7.26 \mathrm{nN} \cdot \mathrm{nm} / \mathrm{rad}^{2}$ \\
\hline Longitudinal torsion & $k_{\text {longitudinal }}^{\text {torsion }}\left(k_{1}^{\tau}\right)$ & $0.145 \mathrm{nN} \cdot \mathrm{nm} / \mathrm{rad}^{2}$ \\
\hline Helical tensile & $k_{\text {helical }}^{\text {stretching }}\left(k_{2}^{r}\right)$ & $0.890 \mathrm{nN} / \mathrm{nm}$ \\
\hline Helical bending & $k_{\text {helical }}^{\text {bending }}\left(k_{2}^{\varphi}\right)$ & $2.43 \mathrm{nN} \cdot \mathrm{nm} / \mathrm{rad}^{2}$ \\
\hline Helical torsion & $k_{\text {helical }}^{\text {torsion }}\left(k_{2}^{\tau}\right)$ & $0.0486 \mathrm{nN} \cdot \mathrm{nm} / \mathrm{rad}^{2}$ \\
\hline
\end{tabular}

\subsubsection{Validation of F-actin MSM model}

Based on the MSM and the obtained force constants, we performed tensile, 
bending and torsion tests for F-actin. The goal is to calculate the corresponding elastic properties of F-actin and examine the effect of actin structure on the properties. In measuring Young modulus, one end of the F-actin was fixed, i.e., all degree of freedom was constrained to zero, while an external force $F_{1}$ is applied to the other end which is pinned to a roller free to move in longitudinal direction (Fig. 2.5(a)). The value of the Young's modulus $Y$ can then be obtained by $Y=\frac{F_{1} / A_{0}}{\Delta L / L_{0}}$, in which $A_{0}$ is the area of the cross-section, $L_{0}$ is the initial length and $\Delta L$ is the elongation in the axial direction. The torsion simulation was also performed for the F-actin (Fig. 2.5(b)), where cantilevered F-actin was considered and a torsional moment $M$ was applied to the monomer of the free end. The torsional rigidity $\kappa^{T}$ was computed as $\kappa^{T}=M L_{0} / \gamma_{\text {tor }}$ [218] where $\gamma_{\text {tor }}$ was the torsional angle generated by M. A cantilevered F-actin was also considered in the bending test as shown in Fig. 2.5(c). Here the bending stiffness $(E I)$ is calculated by $(E I)=F_{2} L_{0}^{3} /(3 w)$ [218] where $F_{2}$ is the transverse force on and $w$ is the resulted deflection of the free end.

(a)

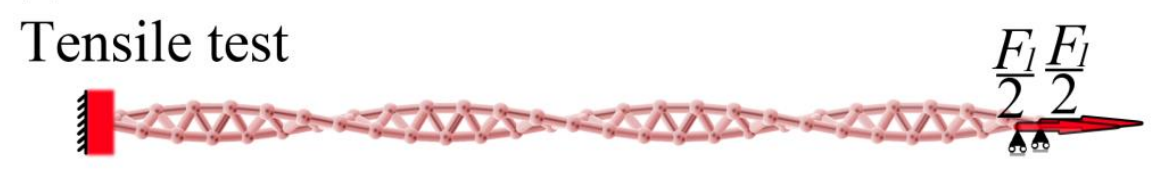

(b)

\section{Torsion simulation}

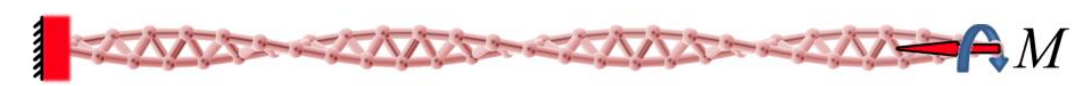

(c)

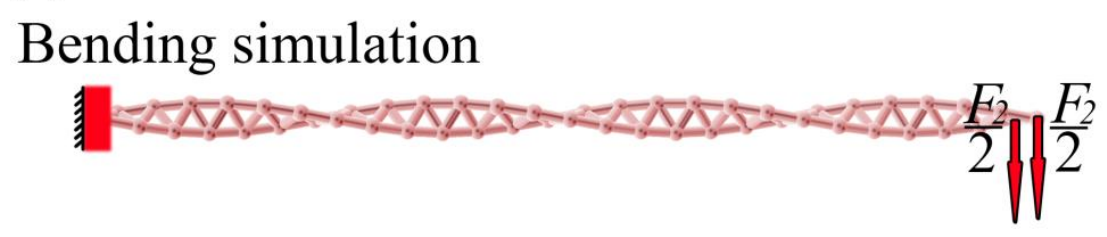

Figure 2.5 Experimental setup in the MSM simulations for (a) tensile, (b) torsion and (c) bending tests of a cantilevered F-actin.

Based on the MSM and the achieved force constants, tensile tests were performed for F-actins. The length of the simulated F-actins ranges from 500 to $50000 \mathrm{~nm}$, while their angular separation equals to $166.15^{\circ}$ or $167.14^{\circ}$. The obtained 
Young's modulus was plotted in Fig. 2.6(a) against the length. It can be seen from the figure that $Y$ decreases slightly with growing length for relatively short F-actins with length smaller than $5000 \mathrm{~nm}$. The length dependence however decreases with the rising length and vanishes when the length is greater than a critical value of 10000 nm. The effect of the angular separation is also observed, i.e., when the angle decreases from $167.14^{\circ}$ to $166.15^{\circ}$ the Young's modulus changes slightly from 1.922 $\mathrm{GPa}$ to $1.915 \mathrm{GPa}$. The average Youngs modulus obtained here was about $1.92 \mathrm{GPa}$, in good agreement with the values 1.8 to $2.6 \mathrm{GPa}$ reported in the literature [11, 197-199].

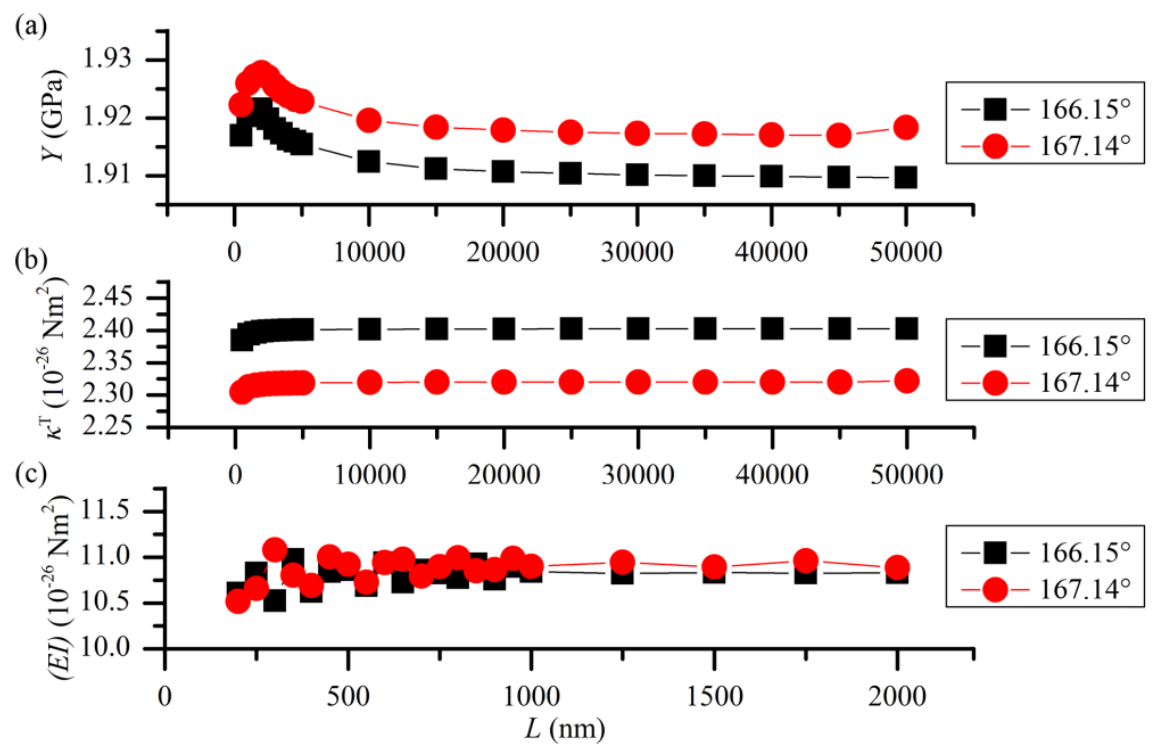

Figure 2.6 The length dependence of (a) Young's modulus $Y$, (b) torsional rigidity $\kappa^{T}$ and (c) flexural rigidity $(E I)$ calculated for F-actins with angular separation of $166.15^{\circ}$ and $167.14^{\circ}$, respectively.

The torsional rigidity $\kappa^{T}$ of F-actins is also calculated for the F-actins with different angular separations. For a given $L$ the $166.15^{\circ} \mathrm{F}$-actins exhibits $\kappa^{T}$ higher than that of the $167.14^{\circ} \mathrm{F}$-actins. The variation of $\kappa^{T}$ however is small $(<2.2 \%)$ leading to an average value around $2.36 \times 10^{-26} \mathrm{Nm}^{2}$ right in the range of $\left[0.2 \times 10^{-26}\right.$, $\left.8.5 \times 10^{-26} \mathrm{Nm}^{2}\right]$ achieved in the literature [18, 182, 200-213].

The flexural rigidity $(E I)$ is an important structural property of F-actins which measures their ability to resist the bending deformation and the structural instability 
of F-actins. In particular, the substantial length-dependence of (EI) was experimentally observed for MTs [20]. Similar feature was also reported for short in silico MTs whose length is smaller than $400 \mathrm{~nm}$ [77]. It is thus of great interest to examine the unique feature of $(E I)$ and calculate its value for the F-actins. To this end, the MSM was used to perform bending tests for the cantilevered F-actins with the length ranging from $200 \mathrm{~nm}$ to $2000 \mathrm{~nm}$. The results were shown in Fig. 2.6(c) which indicated that though $(E I)$ fluctuated slightly with the length change, there did not exist a clear trend for $(E I)$ to change with the length $L$ or the separation angle. In other words, F-actins behave like Euler beams with nearly constant bending stiffness about $10.84 \times 10^{-26} \mathrm{Nm}^{2}$. This is different from the behaviour of relatively short MTs whose $(E I)$ increases with rising length as a result of the inter-PF sliding [82]. It should also be mentioned that the reported values of $(E I)$ are in the range between $1.7 \times 10^{-26} \mathrm{Nm}^{2}$ and $11 \times 10^{-26} \mathrm{Nm}^{2}[11-18,184,197,214-217]$, which is again in good agreement with the average value $10.84 \times 10^{-26} \mathrm{Nm}^{2}$ calculated in Fig. 2.6(c) based on the present MSM model. Overall, the mechanical properties given by the MSM model for F-actins are in accordance with existing experimental data. This comparison between the present MSM and existing experimental data show clear evidence of the relevance of the MSM model to the mechanics of the F-actins.

It is worth mentioning that the estimation method for the force constants in Table 2.2 is different from the experimental setup in Ref. [69] where an external force is assumed to act at the monomer centre (instead of the residues involved in the interactions between monomers). Under this assumption, the force constants for interaction stretching $k_{\text {new }}^{\text {stretching }}$ can be obtained as follows.

$$
\frac{1}{k_{\text {origin }}^{\text {stretching }}}=\frac{1}{2 k_{\text {monomer }}^{\text {stretching }}}+\frac{1}{k_{\text {new }}^{\text {stretching }}}+\frac{1}{2 k_{\text {monomer }}^{\text {stretching }}}
$$

where $k_{\text {origin }}^{\text {stretching }}$ is the coefficient in Table 2.2 , and $k_{\text {monomer }}^{\text {stretching }}=4.98733 \mathrm{nN} /$ $\mathrm{nm}$ is the force constant for monomer stretching. The values of $Y, \kappa^{T}$ and $(E I)$ were obtained based on this method for a $5 \mu m$-long F-actin $\left(166.15^{\circ}\right)$. The obtained $\kappa^{T}=$ 
$2.43 \times 10^{-26} \mathrm{Nm}^{2}$ and $(E I)=12.47 \times 10^{-26} \mathrm{Nm}^{2}$ are similar to the values shown before, whereas $Y=4.82 \mathrm{GPa}$ achieved here is substantially greater than the $1.92 \mathrm{GPa}$ obtained previously. Since the loading condition considered in the Section 2.3.3 of model development is more practical in experiments, the force constants in Tables 2.2 were used in the present simulations.

\subsection{Summary}

In this section, the MSM model for MT was introduced firstly. The equivalency of the MT structure and its MSM model can be established when the energy of monomer interactions deformations and the energy of the beam deformations are equal, which leads to a relationship between the force constants of the interactions and the stiffnesses of the space beams. The MSM model enjoys highly improved efficiency and expanded the scope of the research as compared with formidable MD simulations and difficult nanoscale experiments.

MD simulations were then performed to obtain the force constants between the monomers of F-actin. Subsequently, an MSM model was first obtained for F-actin based on the structural mechanics theory and the force constants obtained. Based on the MSM, the effect of the structure on the elastic modulus and structural stiffnesses was investigated for F-actin. Meanwhile, the validation of F-actin MSM model can be performed. The obtained average effective Young's modulus $Y=1.92 \mathrm{GPa}$, torsional stiffness $\kappa^{T}=2.36 \times 10^{-26} \mathrm{Nm}^{2}$, and bending stiffness $(E I)=10.84 \times 10^{-26} \mathrm{Nm}^{2}$ were found to be in good agreement with existing experimental data. The results demonstrated the reliability and robustness of the present MSM model in characterising mechanical behaviour of F-actins. Herein, it is highly expected that the present MSM model can be further extended to more complex filamentous systems and thus, is able to expand the scope of research to the higher order cytoskeletal structures composed of cross-linked F-actin bundles, such as the stress fibres or actin meshwork. 
However, there still exists room for improvements about the MSM models. For instance, it was reported that the accuracy of the model depends on the loading modes and the model should be used carefully when the non-linear large deformation is considered [219]. Thus, future efforts could be invested in the following aspects to improve the MSM model. 1. The force constants could be optimized for different loading situations and validated through the experimental data. 2. As far as a case with non-linear large deformation is considered, the non-linear feature could be achieved through various optimisations on the model. For example, the strain-dependent constants for basic elements, the additional structural elements between the two nodes of a beam to represent the nonlinear feature, etc. 3 . Since the nano-filaments are influenced by the heat in the environment, it is necessary to include heat induced strain in the model.

In spite of this, there is no doubt that the MSM model has the proven ability to correlate MT structures to the elastic properties [77], mechanical behaviour [78, 167] at relative small deformation. Thus it could be efficiently applied in studies such as inter-PF sliding, eigenvalue buckling/vibration analysis, small amplitude vibration, etc. 


\section{Chapter 3 Protofilament sliding and its effects on MT mechanics}

\subsection{Introduction}

The mechanics of MTs $[11,61,63,79,159-163]$ has been studied extensively in the last two decades, where the length-dependency of equivalent bending stiffness $(E I)_{\mathrm{eq}}$ was captured as a unique feature of MTs and interpreted primarily via the CMMs.

The Euler beam (EB) model was the first CMM used for MTs [11, 51, 161, 220-223]. In 1993, Gittes et al. measured (EI) $)_{\mathrm{eq}}$ for MTs by fitting the beam model to experiments [11]. In 2002, using this technique Kis et al. [21] first reported the length-dependence of $(E I)_{\mathrm{eq}}$ for MTs and attributed it to their low shear modulus $G$. This theory [21] was then used by Kasas et al. to study the effect of anisotropy on MTs via the FE method [65]. Pampaloni et al. also employed the theory to understand the length-dependent $(E I)_{\mathrm{eq}}$ achieved experimentally [20].

In 2006, Ru's group developed an orthotropic shell model for MTs [37, 59] and later compared it with the EB model [61]. The length-dependent $(E I)_{\mathrm{eq}}$ was found to be a result of the extremely low $G$ relative to the axial Young's modulus [61]. Subsequently, Ru and his co-workers [62, 224] confirmed the relevance of the Timoshenko beam (TB) model to MTs by comparing it with the shell model $[37,59]$. The length-dependence of $(E I)_{\mathrm{eq}}$ was also predicted by the TB model via the low $G$-induced transverse shear. In addition, a higher order shear deformation theory was used by Tounsi et.al. [160] to understand the length-dependent $(E I)_{\text {eq }}$ of MTs. This unique feature was also explained by Gao et. al., [63] and Fu and Zhang [225] via the nonlocal elasticity and the couple stress theory, respectively.

A CMM or an improved-CMM $[75,76]$ is often chosen by researchers for a nanostructure primarily due to the similarity between their overall geometric configurations. However, the deformation mechanisms of discrete nanostructures 
may not be correctly reflected by that of a continuous body. This, in fact, forms a fundamental issue in nanomechanics. Specifically, the inter-PF sliding was observed experimentally for MTs $[49,53]$, which originates from the weak inter-PF interaction and is thought to be responsible for the length-dependent $(E I)_{e q}[20,21,73]$. Effort is thus required to further confirm this theory and examine the relevance of the CMMs to the inter-PF sliding of MTs.

The present chapter aims to investigate this issue for the classical and nonlocal beam models $[21,61,63,65,160,225]$. In doing this, an MSM model was employed to characterise the inter-PF sliding $[49,53,82]$ and compared with the beam models in studying the vibration and bending of MTs. The idea is to examine whether the effects of inter-PF sliding can be captured by the continuum beam and nonlocal mechanics theories. The MSM model shows proven ability to correlate MT structures to the elastic properties [77], mechanical behaviour [78, 80, 167] and particularly, the inter-PF sliding [82]. In this chapter the MSM related characterising techniques will be introduced, the numerical results obtained by the model will be presented and a critical analysis based on the results will be carried out.

\subsection{Characterising techniques for MTs}

\subsubsection{The vibration and static deformation of an MT with MSM model}

The vibration equation of the above frame structure is as follows [226, 227]

$$
\mathbf{M} \ddot{\chi}+\mathbf{K} \chi=\mathbf{0}
$$

where $\mathbf{M}$ denotes the global mass matrices, $\mathbf{K}$ denotes the stiffness matrices, $\ddot{\chi}$ denotes the acceleration vector and $\chi$ denotes the nodal displacement vector. For the details of $\mathbf{M}$ and $\mathbf{K}$ readers may refer to Refs. [77, 226, 227]. The vibration modes and frequency $f=\omega / 2 \pi$ can be obtained by solving the eigenvalue problem below [78] via the Block Lanczos algorithm.

$$
\left(\mathbf{K}-\omega^{2} \mathbf{M}\right) \chi=\mathbf{0}
$$


For the static deformation of an MT, the nodal displacements can be calculated for the frame structures of MTs via the stiffness matrix method based on the following equation

$$
\mathbf{K u}=\mathbf{F}
$$

where $\mathbf{u}$ is the global nodal displacements and $\mathbf{F}$ is the nodal forces acting on the boundary of an MT. Solving Eq. 3.3 gives the nodal displacements of the individual nodes and thus the deformation of MTs. This MSM technique was efficiently used in characterising the elastic properties [77], buckling behaviour [167] and free vibration of MTs [78]. It is found to be in good agreement with experiments and other simulations [77], and able to reflect the effect of the inter-PF sliding on MT deformation [82].

In $[69,70]$, MDSs were performed to measure the force constants for MTs., $k_{I}^{r}=3 \mathrm{nN} / \mathrm{nm}, k_{I}^{\varphi}=2 \mathrm{nN} \cdot \mathrm{nm}$ and $k_{I}^{\tau}=0.04 \mathrm{nN} \cdot \mathrm{nm}$ were obtained for the intra-PF $\alpha \beta$ bonds, and $k_{2}^{r}=14 \mathrm{nN} / \mathrm{nm}, k_{2}^{\varphi}=8.5 \mathrm{nN} \cdot \mathrm{nm}$ and $k_{2}^{\tau}=0.17 \mathrm{nN} \cdot \mathrm{nm}$ were calculated for the inter-PF $\alpha \alpha(\beta \beta)$ bonds. However, in the literature [21, 61, 72, 179, 228] large discrepancy (six orders of magnitude different) is found in measuring the shear modulus $G$ that is primarily determined by the inter-PF bonds [20, 82]. Accordingly, in this study while the above intra-PF force constants were used, those of the inter-PF bonds considered vary in a wide range, i.e., $k_{2}^{r}=14 \Omega \mathrm{nN} / \mathrm{nm}, k_{2}^{\varphi}=8.5 \Omega n N \cdot \mathrm{nm}$ and $k_{2}^{\tau}=0.17 \Omega n N \cdot \mathrm{nm}$, where the coefficient $\Omega$ ranges from $10^{-4}$ to $10^{2}$ and alters the effect of the inter-PF sliding [82]. These $\Omega$ values were selected as they can return a range of shear modulus $G$ in accordance with the values reported in the literature. Also, it should be pointed out here that the present MSM technique is applicable only for small deformation of MTs. No matter what $\Omega$ value is considered, this condition can be satisfied by considering a relatively low external load or small vibration amplitude.

\subsubsection{Shear modulus and bending stiffness measurement}


In this study, the MSM simulations were performed to measure the mechanical properties of the 13-3MTs. The MSM simulations and the calculations in the present chapter were carried out with a desktop computer with 4 cores. The boundary conditions and loading conditions considered in the MSM simulations were introduced below and illustrated in detail in Fig. 3.1.

As shown in Fig. 3.1(a), torsional deformation was obtained for the cantilevered MT by applying circumferential force $F_{c}$ on each node of the free end. The other end was fixed by imposing zero degree of freedom on the nodes very close to the fixed end (their axial distance to the end is less than $3 a_{m}$ where $a_{m}$ is subunit repeat along PFs). The shear modulus $G$ can then be calculated by $F_{c} R L /\left(\gamma J_{0}\right)$, where $L$ is the unconstrained length of the MT, $R$ is the radius of MT, $\gamma$ is the torsional angle that is measured in the MSM simulations and $J_{0}$ is the polar moment of inertia $\left(J_{0}=(\pi / 32)\left[\left(2 R+t_{m}\right)^{4}-\left(2 R-t_{m}\right)^{4}\right]\right.$ and the effective thickness of MTs $t_{m}=$ $2.8 \mathrm{~nm}$ [72]). In general, anisotropic MTs may have different shear moduli in circumferential (torsional) and axial directions. However, the more detailed study

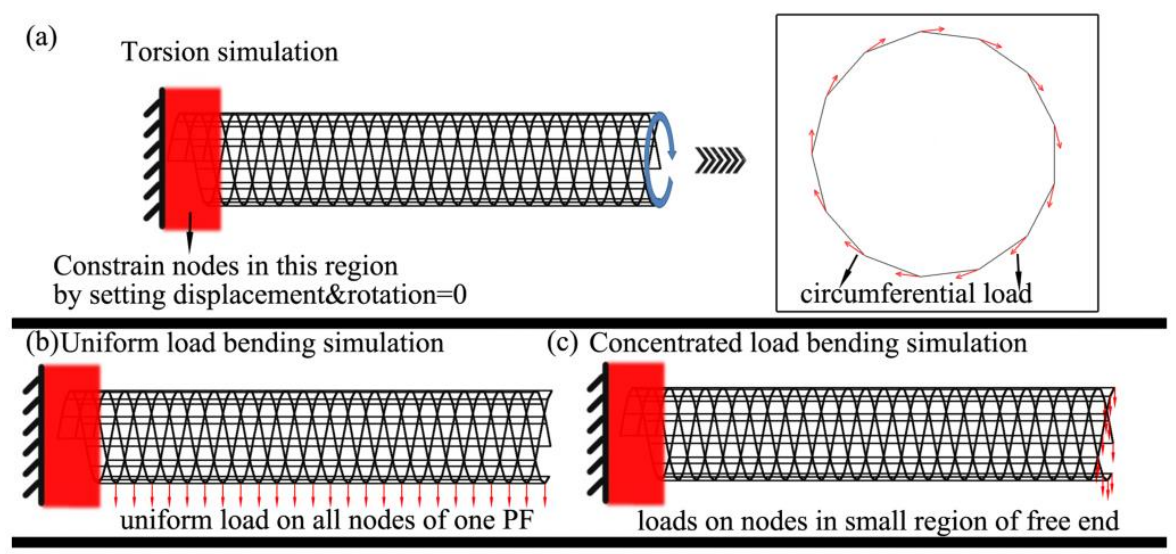

(d) Vibration simulation

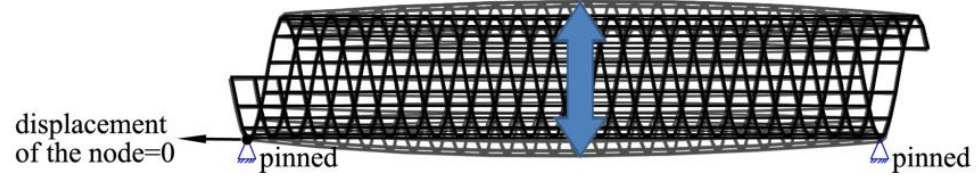

Figure 3.1 Experimental setup in the MSM simulations for (a) torsion, (b) the bending of a cantilevered MT subject to a distributed force, (c) the bending of a cantilevered MT due to a concentrated load on the free end and (d) the vibration of a simply supported MT

based on the present MSM model (not included in the thesis) showed that the two 
shear moduli exhibit the similar trend to change with $\Omega$ and their values associated with a given $\Omega$ are quite close to each other. Thus in the present study, the circumfernetial shear modulus $G$ obtained in the torsion test was used to represent the axial shear modulus and employed in the beam models.

In addition, bending of cantilevered 13-3MTs was achieved under two loading conditions. First a uniformly distributed transverse force $q_{0}(\mathrm{~N} / \mathrm{m})$ was achieved on the MT by applying a transverse force $q_{n}=q_{0} L / N_{u-n o d e s}$ on each node of the bottom PF (Fig. 3.1(b)). Here $N_{u \text {-nodes }}$ is the number of the loaded nodes. The transverse deflection $w_{\max }$ of the free end and the distributed force $q_{0}$ can be measured in the MSM simulations. Thus, $(E I)_{e q}$ of the MT can be calculated based on the EB (Eq. 3.4) and TB (Eq. 3.5) theories, respectively [229].

$$
\begin{gathered}
w_{\text {max }}=\frac{q_{0} L^{4}}{8(E I)_{e q}} \\
w_{\text {max }}=\frac{q_{0} L^{4}}{8(E I)_{e q}}\left(1+\frac{4(E I)_{e q}}{G A_{0} K_{S} L^{2}}\right)
\end{gathered}
$$

Here $A_{0}$ is the area of cross section; $K_{\mathrm{s}}=0.72$ is the shear correction coefficient [20, 72, 77]. Alternatively, a concentrated transverse force $F_{c}$ on the free end can be generated by applying a force $F_{n c}=F_{c} / N_{c \text {-nodes }}$ on the nodes whose axial distance to the free end is less than $3 a_{m}$ (Fig. 3.1(c)). (EI) $)_{e q}$ of the MT can be obtained via the $E B$ (Eq. 3.6) and $T B$ (Eq.3.7), respectively [230].

$$
\begin{gathered}
w_{\max }=\frac{F_{c} L^{3}}{3(E I)_{e q}} \\
w_{\text {max }}=\frac{F_{c} L^{3}}{3(E I)_{e q}}+\frac{F_{C} L}{G A_{0} K_{S}}
\end{gathered}
$$

Additionally, simulations were performed for the transverse vibration of the simply supported MTs. The end conditions were achieved by fixing one node on each end of the MT (Fig. 3.1(d)). Here the angular vibration frequency $\omega$ can be measured in MSM simulations and (EI) eq can be obtained based on EB (Eq. 3.8) and TB (Eq. 3.9) theories, respectively [229, 231].

$$
\omega=\left(\frac{n \pi}{L}\right)^{2}\left(\frac{(E I)_{e q}}{m_{0}}\right)^{1 / 2}
$$




$$
\omega=\left(\frac{n \pi}{L}\right)^{2}\left(\frac{(E I)_{e q}}{m_{0}\left(1+\frac{n^{2} \pi^{2}(E I) e q}{G A_{0} K_{s} L^{2}}\right)}\right)^{1 / 2}
$$

Here it is noticed that the $\Omega$-dependence of $(E I)_{e q}$ can be obtained via $G(\Omega), w_{\max }(\Omega)$ and $\omega(\Omega)$ in Eqs. 3.4 to 3.9 .

\subsubsection{Nonlocal coefficient measurement}

When the size of a structure miniaturizes across the length scale, one would see the changes in the constitutive relations of the material in the structure. For a bulk material, the stresses of a reference point are only a function of the strains at the same point. However, for a nanoscale material, the stresses of a reference point may be determined by the strains of all points in the domain occupied by the nanomaterial $[232,233]$. Previously effort was made to study the bending and vibration behaviour of MTs based on nonlocal theory $[234,235]$. In particular, the length-dependence of $(E I)_{\text {eq }}$ achieved for MTs was interpreted based on the nonlocal beam models developed by incorporating the non-local constitutive relations into the classical beam theories [63]. For a cantilevered MT the bending deflection $w_{\max }$ of the free end was obtained below based on the nonlocal EB and TB models when a uniformly distributed force $q_{0}$ is applied $[229,231]$

$$
\begin{gathered}
w_{\max }=\frac{q_{0} L^{4}}{8(E I)_{e q}}\left(1-\frac{4\left(e_{0} a\right)^{2}}{L^{2}}\right) \\
w_{\max }=\frac{q_{0} L^{4}}{8(E I)_{e q}}\left(1+\frac{4(E I)_{e q}}{G A_{0} K_{S} L^{2}}-\frac{4\left(e_{0} a\right)^{2}}{L^{2}}\right)
\end{gathered}
$$

In addition, the angular vibration frequency $\omega$ of the simply supported MT given by the nonlocal EB and TB models, respectively, are shown below [229, 231]

$$
\begin{gathered}
\omega=\left(\frac{n \pi}{L}\right)^{2}\left(\frac{(E I)_{e q}}{m_{0}\left(1+\frac{n^{2} \pi^{2}\left(e_{0} a\right)^{2}}{L^{2}}\right)}\right)^{1 / 2} \\
\omega=\left(\frac{n \pi}{L}\right)^{2}\left(\frac{(E I)_{e q}}{m_{0}\left(1+\frac{n^{2} \pi^{2}(E I)_{e q}}{G A_{0} K_{S} L^{2}}\right)\left(1+\frac{n^{2} \pi^{2}\left(e_{0} a\right)^{2}}{L^{2}}\right)}\right)^{1 / 2}
\end{gathered}
$$

In Eqs. 3.10 to 3.13, the nonlocal effect is characterised by the coefficients $e_{0} a$, where $e_{0}$ is considered as a material constant that can be determined in experiments 
or the atomistic simulations, and $a$ is an internal characteristics length, e.g. lattice parameter, granular size, or the distance between $\mathrm{C}-\mathrm{C}$ bonds for CNTs [63]. The values of $e_{0} a(\Omega)$ can be calculated by using Eqs. 3.10 to 3.13 once $w_{\max }(\Omega), \omega(\Omega)$ and $G(\Omega)$ are determined in the MSM simulations. Here the effect of the inter-PF sliding is explained exclusively by the nonlocal effect, i.e., $e_{0} a$. Thus, in this study the constant $(E I)_{\mathrm{eq}}$ was obtained when there is no significant inter-PF sliding, i.e., $\Omega>$ 1 , is assumed for the MTs.

\subsection{A coupled molecular and continuum mechanics study for MT deformations}

As mentioned in Sec. 3.1, different continuum mechanics theories were used to investigate the deformation of MTs. The length-dependent $(E I)_{\mathrm{eq}}$ was achieved and thought to be a result of the shear deformation or the non-local constitutive relations of MTs. Herein, an attempt was made to examine whether those effects proposed in the framework of the continuum mechanics theory are able to correctly reflect the deformation mechanisms of discrete MT structures.

\subsubsection{Inter-PF sliding of bent MTs}

In this section, we investigated the effect of the inter-PF sliding on the bending deformation of MTs. To this end, we bent a cantilevered 13-3MT by applying a concentrated force on the free end. Here the effect of the possible inter-PF sliding was altered intentionally by varying the stiffness of the inter-PF bonds in a broad range, i.e., the coefficient $\Omega$ changes from $10^{-2}$ to $10^{2}$. The snapshots of the initial position (the undeformed configuration) and the final position (the bent configuration with the maximum transverse deflection) were shown in Fig. 3.2 for the MTs with $\Omega$ equal to $10^{2}, 10^{1}, 10^{0}, 10^{-1}$ and $10^{-2}$, respectively. The illustrated displacements in the snapshots were enlarged 100-fold to reveal the differences. The transverse deflection of the free end is found to increases when $\Omega$ decreases or the inter-PF bonds become 
softer. However, it rises only slightly when $\Omega$ declines from $10^{2}$ to $10^{0}$, i.e., the inter-PF bonds are relatively stiff. The growth becomes more significant at $\Omega=10^{-1}$ and turns out to be large as $\Omega$ reaches $10^{-2}$ or the inter-PF bonds become very soft.

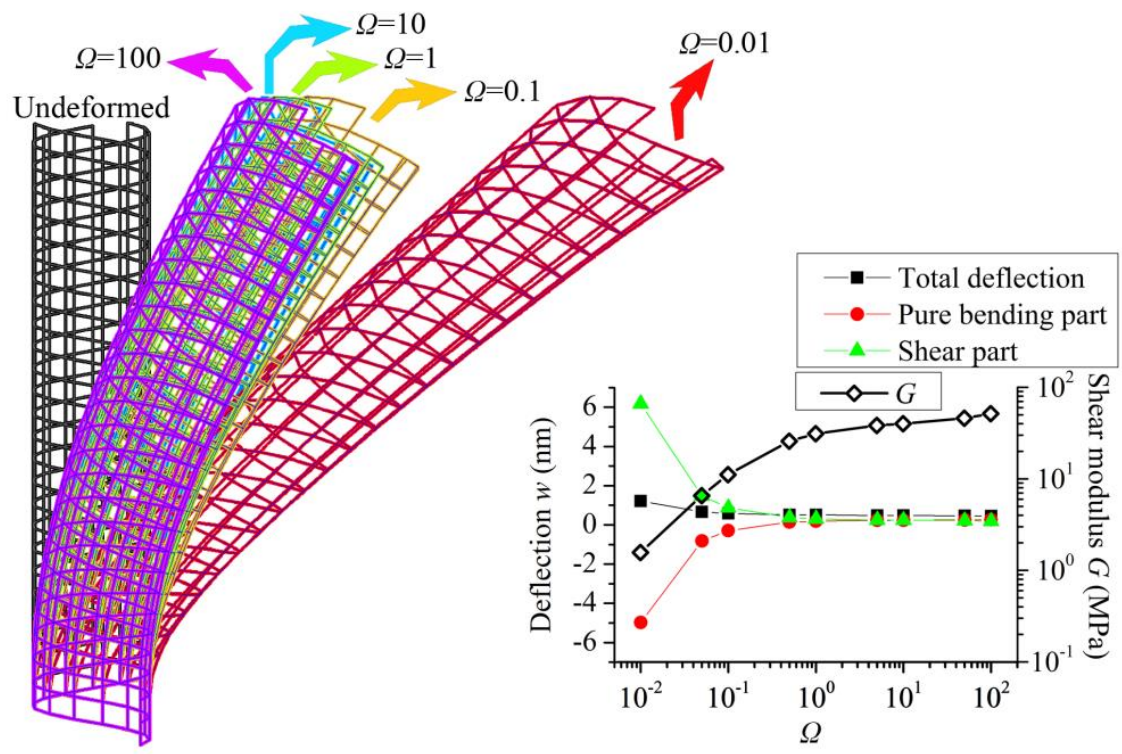

Figure 3.2 The initial position of an undeformed 13-3MT and the final position of the bent MT structures with $\Omega=0.01,0.1,1,10$ and 100, respectively. The illustrated displacements in the snapshots were enlarged 100-fold to reveal the differences. The inset shows the $\Omega$-dependence of the shear modulus $G$ (diamonds), the total bending deflection of the free end (squares), the deflection due to pure bending (circles) and the one resulting from the shear deformation (triangles). The deflections were given by the TB model by using the values of $G$ shown in the inset

In the meantime, we calculated the shear modulus $G$ introduced for MTs in [77]. The $\Omega$-dependency of $G$ was plotted in the inset of Fig. 3.2 where $G$ decreases with decreasing $\Omega$. Specifically, consistent with the above deflection change, $G$ varies only by a few times when $\Omega$ falls in the range of $\left[10^{0}, 10^{2}\right]$. It however drops abruptly by one to two orders of magnitude when $\Omega$ declines from $10^{0}$ to $10^{-2}$. Thus, the stiffness of the inter-PF bond stiffness can be approximately measured by the shear modulus $G$ quantifying the shear deformation resistance of MTs.

In addition, it was also seen from Fig. 3.2 that at $\Omega=10^{2}$, i.e., the inter-PF bond stiffness or the shear modulus $G$ is relatively high, the MT bends like an EB with the central axis (or the neutral axis) nearly perpendicular to the cross-sections. This situation remains nearly unchanged as $\Omega$ reduces from $10^{2}$ to $10^{\circ}$ and the transverse 
deflection grows slightly. In contrast, when $\Omega$ decreases to $10^{-2}$, i.e., the inter-PF bond stiffness or $G$ is one to two orders of magnitude lower, the inter-PF sliding or shear deformation can be clearly observed for the MT where the central axis is no longer perpendicular to the cross-section. It follows that at $\Omega>10^{0}$, the small transverse deflection in Fig. 3.2 is primarily a result of the pure bending of the MT. By contrast, at $\Omega=10^{-2}$ the deflection increases greatly due to the inter-PF sliding or the shear deformation of the MT.

Based on the above MSM simulations it can be concluded that the soft inter-PF bond will lead to the large inter-PF sliding or the shear deformation, and thus additional (or greater) transverse deflection of MT structures. The stiffness of the inter-PF bonds or the resistance to shear deformation of MTs is measured by the shear modulus $G$ that can be obtained in the MSM simulations. This theory is qualitatively similar to the concepts of the proposed CMMs [20, 21, 37, 59, 61, 62, 160, 224] where the shear deformation is considered for MTs. For example, Eq. 3.7 obtained based on the TB model gives the transverse deflections due to pure bending $\frac{F_{C} L^{3}}{3(E I)_{e q}}$ and the shear deformation $\frac{F_{c} L}{G A_{0} K_{S}}$, respectively. Thus Eq. 3.7 was employed to quantify the MT deflections due to the pure bending and shear deformation (or the inter-PF sliding). The results were also plotted in the inset of Fig. 3.2 where at $\Omega<$ $10^{0}$, the shear deflection (solid triangles) given by the TB model ( $G$ is measured by the MSM model) is even larger than the total deflection (solid squares) observed in the MSM simulations. This finally leads to an unacceptable negative bending deflection (solid circles) or a negative bending stiffness of the MT. The results suggest that though the TB model is generally in qualitative agreement with the MSM simulations, it may overestimate the effect of the equivalent shear deformation or the inter-PF sliding in some particular cases. This situation thus necessitates a more comprehensive investigation on the relevance of the classical beam models to the mechanical deformations of MTs. 


\subsubsection{Classical beam models for MTs}

In the previous section, the bending of 13-3 MT was studied based on the MSM model and the classical beam theories. The inter-PF sliding of MTs was identified as the physical origin of the shear deformation considered in the TB model for MTs. In this section, an investigation was carried out to further examine the relevance of the beam models to the mechanics of MTs. To this end, the $\Omega$-dependency of $(E I)_{e q}$ was calculated in Fig. 3.3 by fitting the EB and TB models to the MSM simulations on the vibration or bending of MTs. Herein, 13-3 MT structures were considered where the length $L$ is fixed at $\sim 0.85 \mu \mathrm{m}$, i.e., the length-to-diameter ratio $L / D=40$, and $\Omega$ varies between $10^{-4}$ and $10^{2}$. The shear modulus $G$ was also given in Fig. 3.3 to understand the trend of $(E I)_{e q}$.

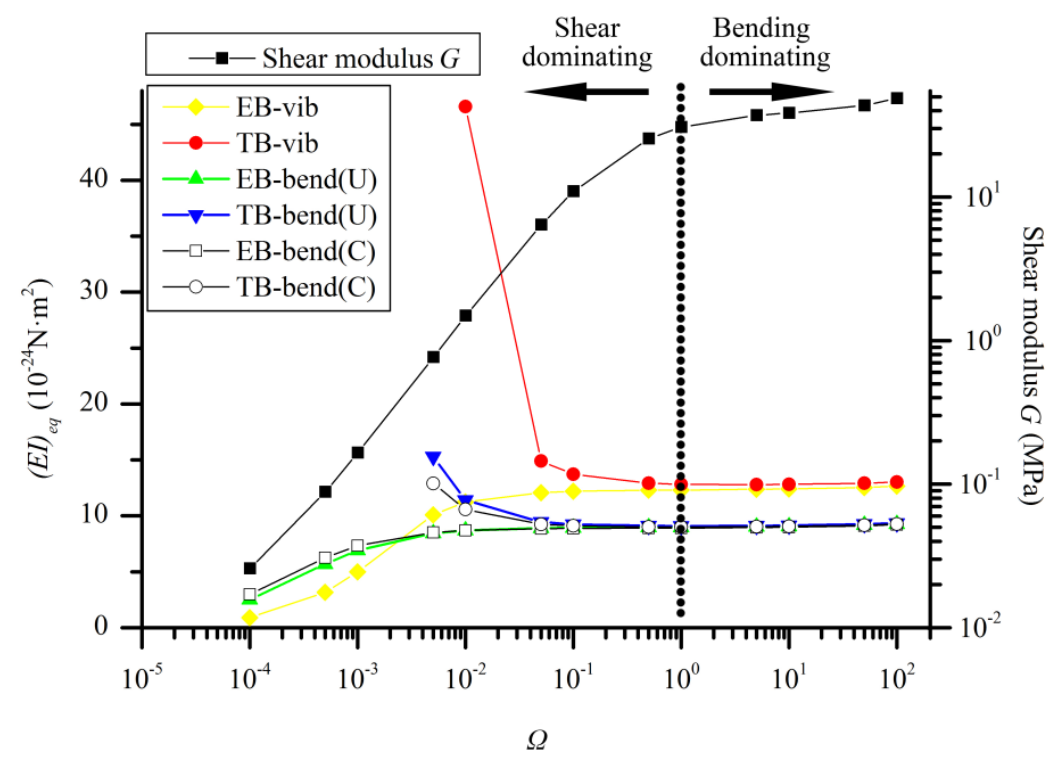

Figure 3.3 The $\Omega$-dependency of the shear modulus $G$ (squares) obtained in the MSM simulations and that of $(E I)_{e q}$ calculated for MT structures with $L / D=40$. $(E I)_{e q}$ obtained for vibrating MTs based on the EB and TB models are represented by diamonds and circles, $(E I)_{e q}$ of bent MTs under a uniform load given by the EB and TB models are denoted by triangles and upside-down triangles, and the one for bent MTs subject to a concentrated load achieved by using the EB and TB models are represented by squares and circles, respectively

In the range $10^{0}<\Omega<10^{2}, G$ in Fig. 3.3 falls in the range of [30.8MPa, 48.8MPa] where, as shown in Sec.3.1, the effect of the inter-PF sliding is very small 
or negligible. Thus, $(E I)_{e q}$ obtained based on the frequency of MT vibration is nearly a constant around $13 \times 10^{-24} \mathrm{Nm}^{2}$. The difference between the EB (solid diamonds) and TB (solid circles) due to shear modulus $G$ was found to be small showing that the MT vibrates like an EB where the effect of the shear deformation or the inter-PF sliding is trivial.

In the range $10^{-4}<\Omega<10^{0}, G$ decreases greatly from around $30.8 \mathrm{MPa}$ to 0.026 MPa as $\Omega$ declines. The $G$-variation of three orders of magnitude is found to be in the same range of $G$ values reported in the literature $[21,61,72,179,228]$. In this process when the inter-PF bonds become softer, the effect of the inter-PF sliding turns out to be more significant leading to more compliant MT structures and thus, a lower frequency. Accordingly, in Fig. 3.3, $(E I)_{e q}$ given by the EB model (solid diamonds) is found to decrease with decreasing $\Omega$. In other words, the EB model interprets the lower frequency due to the enhanced effect of the inter-PF sliding (or increased shear deformation due to lower $G)$ in terms of the decreasing $(E I)_{e q}$. In other words, the EB model is unable to reflect the real deformation mechanisms of the discrete MT structure with softer inter-PF bonds.

In contrast to the EB model, $(E I)_{e q}$ obtained based on the TB model (solid circles) climbs up when $\Omega$ drops from $10^{0}$ to $10^{-4}$. As shown in Sec. 3.1, the TB model is considered to be more relevant to MTs as the shear deformation or $G$ of the TB can quantitatively explain the effect of the inter-PF sliding $[49,53]$. Nevertheless, the $\Omega$-dependence of $(E I)_{e q}$ (solid circles) found in Fig. 3.3 is not true for the MTs. In fact, the MSM simulations (the results are not shown here) showed that the axial Young's modulus $E(\approx 0.8 G P$ a) (represented as $E$ instead of $Y$ at here) and the second moment of inertia $I$ are not sensitive to the change in the inter-PF bond stiffness or the coefficient $\Omega$. In other words, $(E I)_{e q}$ defined as the product of $E$ and $I$ should be nearly a constant independent of $\Omega$. Thus, the predicted $\Omega$-dependence of $(E I)_{e q}$ suggested again that the TB model overpredicts the softening effect of the inter-PF sliding on MT structures. As a result, $(E I)_{e q}$ of the TB model has to be raised to 
counterbalance the overestimated effect of the inter-PF sliding (or the shear deformation) and keep the obtained frequencies the same as those of the MSM model. This observation is consistent with the one for MT bending in Sec. 3.1.

The $\Omega$-dependence of $(E I)_{e q}$ was also achieved in Fig. 3.3 via the bending tests in the MSM simulations. The results for the MTs subject to a distributed force (Fig. 3.1(b)) and forces on the free end (Fig. 3.1(c)) are nearly the same and qualitatively similar to those obtained via the vibration of the simply supported MTs (Fig. 3.1(d)). The major difference in these two cases is that, at $10^{0}<\Omega<10^{2}$ the constant $(E I)_{e q} \approx$ $9 \times 10^{-24} \mathrm{Nm}^{2}$ obtained in MT bending is lower than $\sim 13 \times 10^{-24} \mathrm{Nm}^{2}$ measured in MT vibration. The discrepancy can be partially attributed to the different boundary conditions considered in the bending and vibration of the MTs. These results thus support the conclusions drawn above based on the $\Omega$-dependence of $(E I)_{e q}$ achieved via MT vibration.

Furthermore, in Fig. 3.4 the $\Omega$-dependency of $(E I)_{e q}$ was calculated for the uniformly loaded bending of 13-3 MT structures whose aspect ratio $L / D$ rises from 10 to 80 . The results were analogous to what was observed in Fig. 3.3, i.e., when $\Omega$ is relatively large and the effect of the inter-PF sliding is small, both the EB and TB models give nearly the same $(E I)_{e q} \approx 9 \times 10^{-24} \mathrm{Nm}^{2}$ independent of $\Omega$ and the aspect ratio $L / D$. In this case the $(E I)_{e q}$ curves given by the two beam models nearly coincide with each other. However, when $\Omega$ decreases the $(E I)_{e q}$ curves of the two beam models bifurcate at a critical value $\Omega_{c r}$ and then, show the reversed trend of $(E I)_{e q}$. The values of $\Omega_{c r}$ decreases from $1 \times 10^{0}$, to $1 \times 10^{-1}$ and $1 \times 10^{-2}$ when the aspect ratio rises from 10 to 30 and 80 . Further increasing the aspect ratio to 160 and 320 leads to less pronounced decreasing trend of $\Omega_{c r}$. These situations considered in Fig. 3.4 are close to the MTs found in cells, which are usually 1 to $10 \mu \mathrm{m}$ long [20] or aspect ratio 40 to 400 . It was found in the figure that $\Omega_{c r}$, i.e., the maximum $\Omega$ value associated with the substantial inter-PF sliding, varies between $5 \times 10^{-2}$ and $1 \times 10^{-3}$. The corresponding $G$ values, as shown in Fig. 3.2, lies in the range of $[0.163 \sim 6.421 \mathrm{MPa}] . \quad$ These results 
suggest that the upper limit of the shear modulus of MTs should be at the order of $1 \mathrm{MPa}$, which is close to the shear modulus 1.4MPa measured in [21]. The higher shear modulus $G$ is unlikely as it would prevent the inter-PF sliding that has already been observed for MTs in the experiments [49, 53, 236].

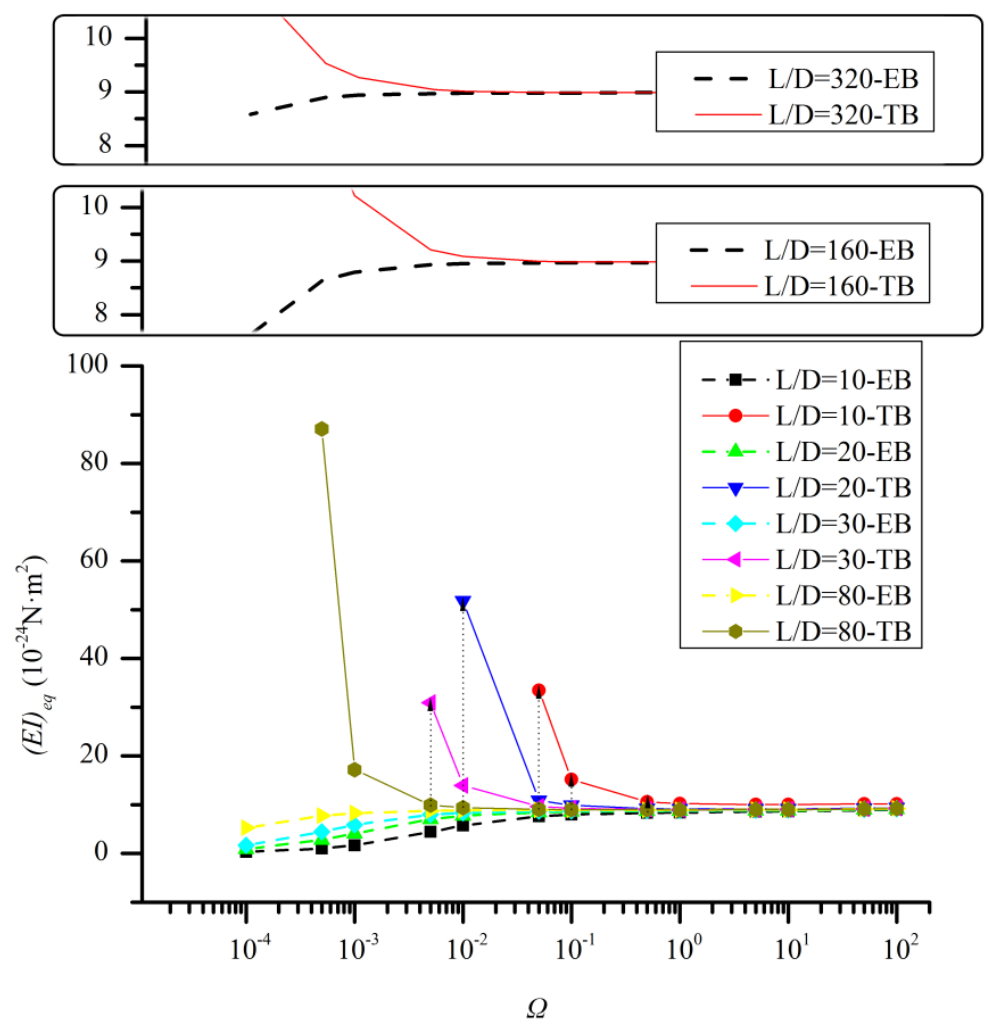

Figure 3.4 The $\Omega$-dependency of $(E I)_{e q}$ obtained for bent MT structures which are subject to a distributed transverse load and possess $L / D$ rising from 10 to 320

It is clearly seen from Fig. 3.4 that, when $\Omega<\Omega_{c r}$, $(E I)_{e q}$ of the MTs exhibit substantial length-dependence (see vertical dotted lines in Fig. 3.4) whereas when $\Omega>$ $\Omega_{c r},(E I)_{e q}$ remains a constant without significant $\Omega$ - and length-dependence. These results further confirm that the inter-PF sliding resulting from the soft inter-PF bonds is the physical origin of the length-dependence of $(E I)_{e q}$ obtained based on the classical beam theories. In other words, the MT structures behave like an EB (or a TB) with a constant $(E I)_{e q}$ when their inter-PF bonds are stiff and the effect of the inter-PF sliding is very small or negligible. However, as far as the soft inter-PF bonds are concerned or the softening effect of the inter-PF sliding becomes substantial, the 
length-dependence of $(E I)_{e q}$ emerges because the EB model is unable to account for the effect of the inter-PF sliding or shear deformation and the TB model overestimates its softening effect.

Here it is clearly seen from the above analyses that, the agreement of a CMM with discrete simulations can be achieved by using the elastic modulus or structural stiffnesses obtained via curve fitting. The identical numerical values, however, do not necessarily confirm the relevance of the CMM to the nanostructure as the curve fitting results may not correctly imitate the physical mechanisms of MT deformations. This is simply due to the distinct deformation mechanisms between a discrete nanostructure and its equivalent continuous body of similar geometric configuration.

\subsubsection{Nonlocal beam models for MTs}

In this section, the nonlocal effect characterised by the nonlocal coefficient $e_{0} a$ was employed to quantify the influence of the inter-PF sliding on MT structures, which, as shown above, can also be measured by the equivalent shear modulus $G$ or the coefficient $\Omega$. The goal is to examine the relevance of the nonlocal theories to the effect of the inter-PF sliding, a unique deformation mechanism of MT structures.

In doing calculations the shear modulus $G$ shown in Fig. 3.3 was used in the TB model, which decreases with declining $\Omega$. On the other hand, constant $(E I)_{e q}$ associated with $\Omega>10^{\circ}$ in Fig. 3.3 was used for both nonlocal EB and TB models as its value (not the curve fitting one) does not change significantly with $\Omega$. Here the $\Omega$-dependence of $\left(e_{0} a\right)^{2}$ was calculated based on Eqs. 3.10 and 3.12 (the nonlocal EB theory) and Eqs. 3.11 and 3.13 (the nonlocal TB theory) in studying the bending and vibration of the MT structures, respectively. The results were plotted graphically in Fig. 3.5.

First let us consider the results obtained from the vibration of simply supported MTs. It was shown in Fig. 3.5 that $\left(e_{0} a\right)^{2}$ achieved based on the EB model (solid squares) decreases from $942106 \mathrm{~nm}^{2}$ to a value close to 0 when $\Omega$ rises from $10^{-4}$ to 
$10^{0}$, i.e., the inter-PF bonds become stiffer and the effect of the inter-PF sliding or the nonlocal effect decreases. As expected, $\left(e_{0} a\right)^{2}$ finally becomes very small when $\Omega$ is further raised from $10^{0}$ to $10^{2}$, showing very small or negligible inter-PF sliding or the nonlocal effect. In this case, as shown in Secs.3.1 and 3.2 the MT structures can be approximately modelled as a classical EB. These seem to suggest that the growing effect of the inter-PF sliding due to softening of the inter-PF bonds can be adequately captured by the nonlocal EB model.

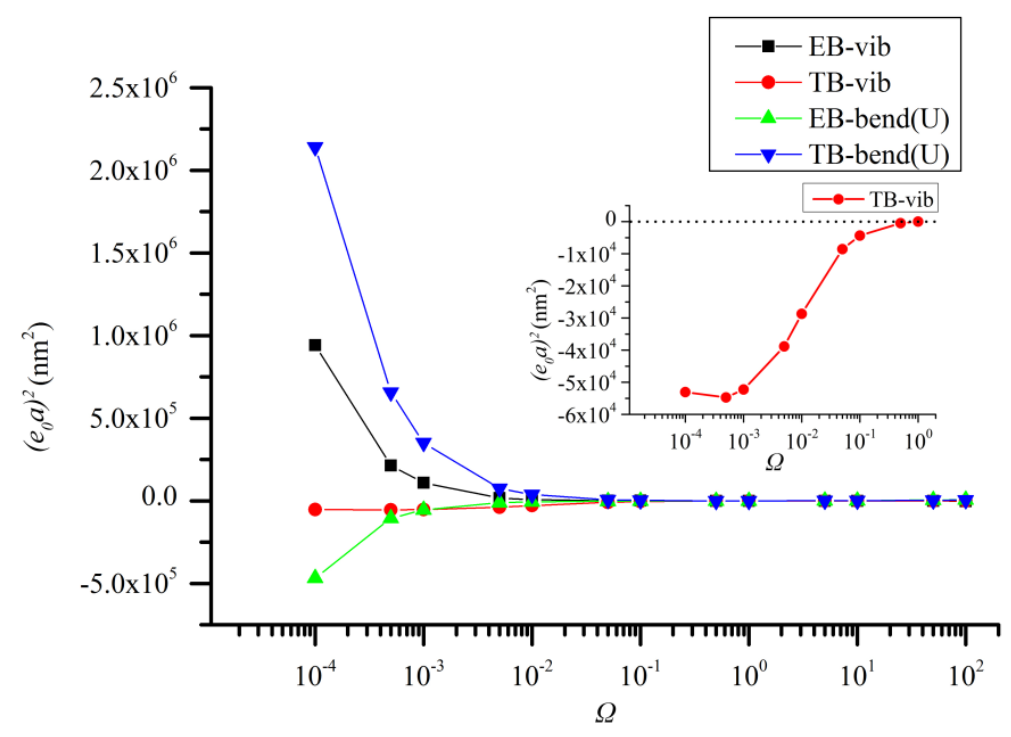

Figure 3.5 The $\Omega$-dependence of $\left(e_{0} a\right)^{2}$ calculated for 13-3MT structures. The data obtained for the vibrating MT structures based on the nonlocal EB and TB models are represented by squares and circles, respectively, and those for the bent MTs by using the nonlocal EB and TB models are denoted by triangles and the upside-down triangles, respectively. Negative values of $\left(e_{0} a\right)^{2}$ shown in the figure indicate the situation where the nonlocal beam model is not relevant for the mechanics of MTs.

On the other hand, $\left(e_{0} a\right)^{2}$ given by the TB model (solid circles) shows an opposite trend in Fig. 3.5 where negative $\left(e_{0} a\right)^{2}$ is found at $\Omega<10^{0}$ (inset) and it approaches 0 at $\Omega>10^{0}$. The latter matches the results of the EB model. The former however is a trivial solution without real physical explanations. As shown in Secs.3.1 and 3.2, the TB model accounts for the inter-PF sliding in terms of the shear deformation, but it overestimates its effect on MT vibration, i.e., the MT frequency given by the TB model is even lower than the one obtained in the MSM simulations. 
Thus, when the nonlocal effect is incorporated into the TB model, negative $\left(e_{0} a\right)^{2}$ is required to upshift the frequency and make it equal to the MSM value. Positive $\left(e_{0} a\right)^{2}$ however signifies the softening nonlocal effect on the simply supported MTs, which further decreases the frequency. It is thus clear that considering both the shear deformation and the nonlocal effect may not lead to a beam model more suitable for MTs than the one with only one of the two effects.

Next we considered the data in Fig. 3.5 collected for the bending of cantilevered MTs. In this case, $\left(e_{0} a\right)^{2}$ of the nonlocal EB model (solid triangles) grows with rising $\Omega$ (Fig. 3.5), and remains negative at $10^{-4}<\Omega<10^{0}$ where the softening effect of the inter-PF sliding is substantial. The trend of $\left(e_{0} a\right)^{2}$ and specifically, the negative $\left(e_{0} a\right)^{2}$ obtained for the cantilevered MTs (solid triangles) are found to be different from those of the simply supported MTs (solid squares). The discrepancy is due to the sensitivity of the nonlocal effect on the end conditions of beams [229]. While it exerts softening influence on the simply supported beams (e.g., a lower frequency given by Eqs. 3.12 and 3.13) it generates stiffening effect on the cantilevered ones (e.g., a smaller bending deflection given by Eqs. 3.10 and 3.11). In contrast to this, the inter-PF sliding always results in a more compliant MT structure with a lower vibration frequency or a larger bending deflection. The meaningless negative $\left(e_{0} a\right)^{2}$ is thus a result of the reverse influence of the nonlocal constitutive relations and the inter-PF sliding on the cantilevered MTs. Thus, the nonlocal EB model, adequate for simply supported MTs as shown above, was found to be unsuitable for the cantilevered MT structures when the inter-PF sliding is substantial. The $\Omega$-dependence of $\left(e_{0} a\right)^{2}$ given by the nonlocal TB model (solid triangles) was also shown in Fig. 3.5 where $\left(e_{0} a\right)^{2}$ grows with decreasing $\Omega$ or increasing softening effect of the inter-PF sliding. Here the stiffening nonlocal effect associated with positive $\left(e_{0} a\right)^{2}$ is again in contradiction with the softening effect of the inter-PF sliding. Thus, the nonlocal beam models are unable to capture the deformation mechanisms of the cantilevered MTs with large inter-PF sliding. 


\subsection{Summary}

MSM simulations were performed to study the bending and vibration of 13-3 MTs. The shear modulus $G$, the bending stiffness $(E I)_{\mathrm{eq}}$ and the nonlocal coefficient $e_{0} a$ were measured for the MT structures based on the MSM model, CMMs and nonlocal mechanics theory. The unique features were achieved and elucidated via the shear deformation or the nonlocal constituent relations.

It is found that the inter-PF sliding may occur for the MT structures in transverse bending or vibration due to the soft inter-PF bonds whose stiffness can be measured roughly by the equivalent shear modulus $G$. When $G$ is in the order of $10 \mathrm{MPa}[72,179]$, the inter-PF interaction is sufficiently strong to largely prevent the adjacent PFs from sliding relative to each other. Thus, an MT deforms as a classical EB with its central line perpendicular to the cross-section and its bending stiffness $(E I)_{\text {eq }}$ independent of the length.

Nevertheless, at $0.01 \mathrm{MPa}<G<10 \mathrm{MPa}[21,61]$ the inter-PF bonds becomes much softer, which yields substantial inter-PF sliding and thus, more flexible MT structures with a lower vibration frequency or a larger bending deflection. In particular, $G$ in the order of $1 \mathrm{MPa}$ can be considered as an upper limit of the possible shear modulus of MTs. It is shown that the EB is unable to reflect this deformation mechanism. The TB model describes the inter-PF sliding via the shear deformation but overestimates its softening effect. These finally yield the length-dependence of $(E I)_{\mathrm{eq}}$ for MTs. In addition, the nonlocal beam models are unable to fully reflect the softening effect of the inter-PF sliding as its effect depends sensitively on the end conditions of MTs.

It is noted that the discrepancy between the MT structures and the proposed continuum mechanics theories is a result of the distinct deformation mechanisms between the discrete MT nanostructure and its equivalent continuous body. 


\section{Chapter 4 Helix structure effects on the mechanics of CSK filaments}

\subsection{Introduction and model definition}

As one of the fundamental structural elements in cells MTs can withstand external load, detect the mechanical changes in the cellular environment and organize the remodelling of the whole CSK [237, 238]. Thus the mechanics of MTs has excited extensive studies in the last two decades [37, 58-60, 82]. Specifically, the vibration of MTs has drawn considerable attention from the communities of nano and biomechanics $[37,58-60,76-78,82]$ as it has the potential to impact on the intra-cellular physiological processes $[71,160,239,240]$, provides a physical mechanism for the novel non-invasive biosensors [241] and facilitates the development of advanced biomimetic nanomaterials, e.g., MT-graphene nanotubes, whose applications rely heavily on MT vibration [242, 243].

Another fundamental issue in MT mechanics is the relation between the MT structures and their mechanical behaviours and properties [244]. This however has not been examined in detail until a recent study on the elastic properties of MTs based on a recently developed MSM model $[77,78,167]$. The possible reason is that the previously used continuum models $[58,60]$ are unable to account for the structural details and the MD simulations [41] are computationally expensive for the analyses of the overall mechanics of MTs. This situation thus provides an impetus for us to further examine the possible structural effect on the vibration of helical MTs based on the MSM model [77, 78, 167], which enjoys highly improved efficiency as compared with MDS and largely enhanced scope relative to the CMMs.

In the second part of the chapter, the MSM model [77] was employed to investigate the beam-like bending vibration for helical MTs with an emphasis on its dependence on the structures and the geometric size of MTs. The vibration analysis for MSM model follows the same method introduced in the Section 3.2.1. To 
examine the effect of structural details (especially the helicity) on MT vibration, MTs with different $N$ and $S$ were considered in the present study. Their structural details were tabulated in Table 2.1 including an imaginary (non-helical) MT with $N=13$ but $S=0$. During these simulations, two different boundary conditions were considered, i.e. (1) two ends of the MTs were fixed and (2) one end is fixed and the other is free. The fixed boundary condition was implemented by imposing the translational restraints $U_{x}=0, U_{y}=0, U_{z}=0 \quad\left(U_{x}, U_{y}\right.$ and $U_{z}$ are displacements in $x, y$ and $z$ directions) and rotational restraints $R O T_{x}=0, R O T_{y}=0, R O T_{z}=0 \quad\left(R O T_{x}, R O T_{y}\right.$, $R O T_{z}$ are rotation angles about $x, y$ and $z$ axes) on the nodes in a region close to MT ends.

On the other hand, the presence of internal forces within the CSK was of major interest in the current research [121]. The ability to sustain tension and compression offers the cell rigidity and maintains its structural stability [157]. It is generally admitted that F-actins could undergo tension while MTs are always compressed [157]. Specifically, in the models of the actin bundles and the tensegrity model of the CSK, F-actins play an important role in resisting tensile forces [245, 246]. Thus, it is interesting to explore the effect of helical structure of F-actin on the mechanical responses in stretching state. The tensile tests for F-actin were performed based on the MSM and the obtained force constants introduced in Section 2.3. The results were presented in Sec. 4.3.

It is worth mentioning that the MSM simulations in the present chapter were carried out with a desktop computer with 4 cores.

\subsection{Three-dimensional transverse vibration of MTs}

In this section, the MSM model introduced in Sec. 4.1 and chapter 2 was employed to perform simulations on the vibration of MTs with different configurations. The focus was placed on the unique features of MT vibration and the effect of structural details on the dynamic behaviours, such as the number of PFs, the 
helix-start number $S$ and the characteristic length.

\subsubsection{D transverse vibration of MTs}

Herein, the transverse vibrations were simulated for long 13-3 MTs (i.e., $N=13$ and $S=3$ ) with two fixed ends and the length-to-diameter ratio $L / D=113$ or the contour length $L=2.4 \mu \mathrm{m}$. Its fourth vibration mode (i.e., the axial half wave number $m=4)$ was shown in a three-dimensional (3D) graph and projected to the three coordinate planes in Fig. 4.1. For the sake of comparison, the fourth mode was also shown for a non-helical 13 MT (i.e., $N=13$ and $S=0$ ) with the same end conditions and the same length. It should be pointed out that the non-helical MT does not exist. It is an MSM model created merely for the comparison.

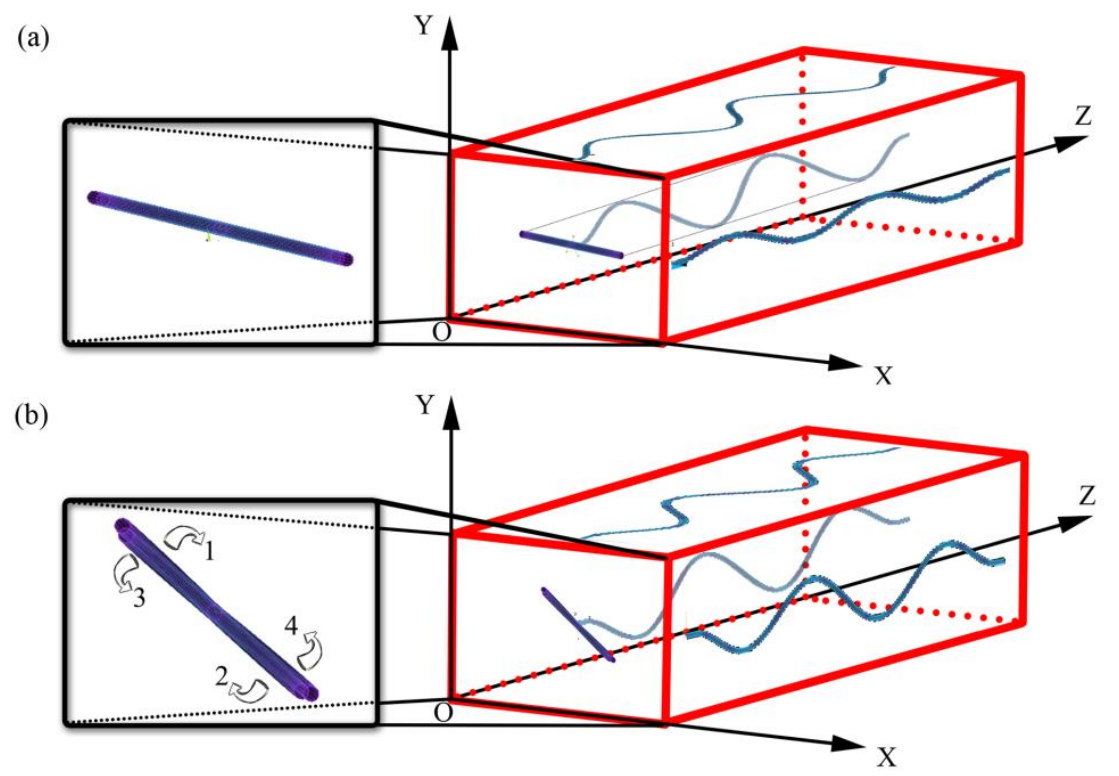

Figure 4.1 Transverse vibration modes $(m=4)$ and its projection to the YOX, YOZ, XOZ planes obtained for (a) an imaginary non-helical 13 MT and (b) a 13-3 MT;

As listed in Table 4.1, the frequencies of the 13-3 MTs and the non-helical MSM model are almost the same, indicating that the helicity did not significantly affect the natural frequency of MTs. Thus, in what follows we focused our attention on the mode shapes of the transverse vibration shown in Fig. 4.1. It is seen from the figure that the vibration modes and their projections in the $\mathrm{YOZ}$ and $\mathrm{XOZ}$ planes are quite similar between the 13-3MT and its non-helical counterpart. On the other hand, a substantial difference was identified in the projections on the YOX plane, i.e., one 
straight line was observed for the non-helical MT, suggesting that the whole tube is vibrating in the same plane. This behaviour is very similar to the one achieved for classical beams. Concurrently, four different straight lines were found in the XOY plane of the 13-3MT, showing that its four half waves are vibrating in four different planes. Thus, Fig. 4.1 showed a 3D transverse vibration for 13-3 MTs.

Table 4.1 Natural frequencies of different vibration modes of models ( $\mathrm{MHz})$

\begin{tabular}{|c|c|c|c|c|}
\hline \multicolumn{3}{|c|}{ Models } & $\begin{array}{c}13-3 \text { MSM } \\
\text { model of MT }\end{array}$ & $\begin{array}{c}\text { Non-helical } \\
\text { MSM model }\end{array}$ \\
\hline \multirow{3}{*}{$\begin{array}{c}\text { Half wave } \\
\text { Numbers }\end{array}$} & $m=1$ & $\lambda / 2 D=113$ & 3.4154 & 3.4156 \\
\cline { 2 - 5 } & $m=2$ & $\lambda / 2 D=56.5$ & 9.4014 & 9.4019 \\
\cline { 2 - 5 } & $m=3$ & $\lambda / 2 D=37.7$ & 18.3960 & 18.3970 \\
\cline { 2 - 5 } & $m=4$ & $\lambda / 2 D=28.25$ & 30.3360 & 30.3390 \\
\hline
\end{tabular}

To confirm the observation in Fig. 4.1 the vibration mode with $m=4$ was further enlarged in Fig. 4.2 for the two types of nanoscale tubules. Herein Fig. 4.2(a) clearly indicated that the 13 MT $(S=0)$ is oscillating in a single plane, i.e., all the four half waves stay in the same oscillation plane. In contrast, Fig. 4.2(b) demonstrated that for 13-3 MTs the half wave plane rotates in an anticlockwise direction. The rotation angles between adjacent planes were denoted by $\xi_{1}, \xi_{2}$ and $\xi_{3}$, respectively. It is understood that bending plays an important role in the transverse vibration of MTs. Thus the spin of the half wave planes suggested the rotation of the bending axis of MT cross-section as the directions of oscillation and the bending axis are always perpendicular to each other. It should be noted that Fig. 4.2(b) only gave a simplified model of the vibration. More detailed study showed that for 13-3MTs individual half waves do not really oscillate in the same plane. As can be seen in Fig. 4.2(c), the anticlockwise rotation of the bending axis actually occurs continuously throughout the whole length of the MT.

To correlate the rotation of the oscillation planes and their bending axis we set up a Cartesian coordinate system on the MT (Fig. 4.2(c)) where the origin coincides with the cross section centre on the left end, $o z$ represents the longitudinal direction and $o y$ shows the bending axis on the left end. Next let us consider the cross section 
at the middle point of the first half wave. The rotation angle $\delta_{1}$ of its bending axis
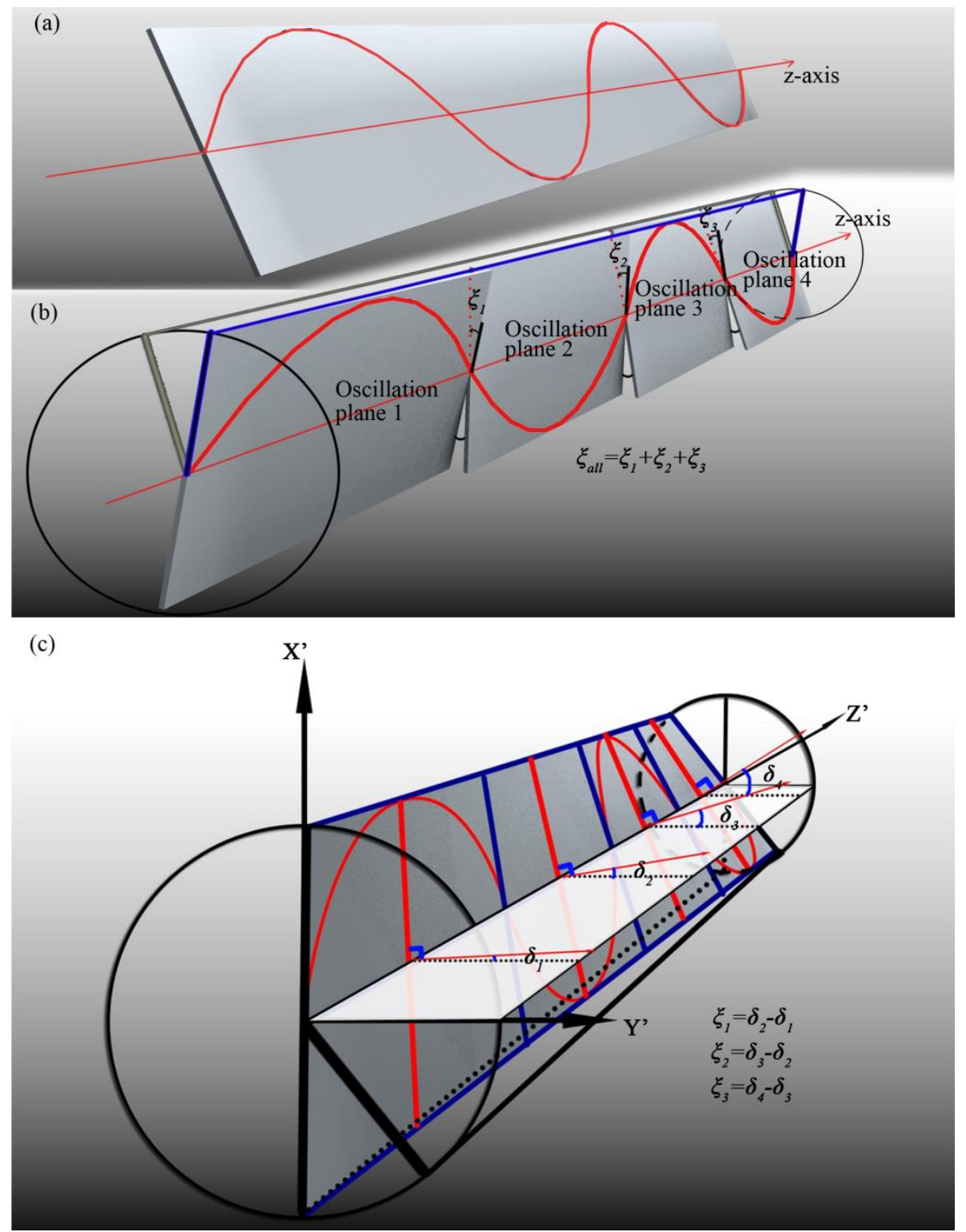

Figure 4.2 The simplified mode shape $(m=4)$ for (a) an imaginary 13MT and (b) a 13-3 MT where the rotation of the half wavelength planes (or oscillation planes) is shown. The real mode shape of the 13-3MT is shown in (c) where the bending axis rotates throughout the whole length of the MT.

relative to the $o y$ was utilized to characterise the average rotation of the $1^{\text {st }}$ half wave plane (or oscillation plane 1 in Fig. 4.2). Subsequently, we defined $\delta_{2}, \delta_{3}$ and $\delta_{4}$ in a similar way for the $2^{\text {nd }}, 3^{\text {rd }}$ and $4^{\text {th }}$ half waves to measure the average rotation of the oscillation planes 2, 3 and 4, respectively (Fig. 4.2(c)). The relative rotation angles $\xi_{1}, \xi_{2}$ and $\xi_{3}$ between the two adjacent oscillation planes shown in Fig. 4.2(b) were 
defined by $\xi_{1}=\delta_{2}-\delta_{1}, \xi_{2}=\delta_{3}-\delta_{2}$ and $\xi_{3}=\delta_{4}-\delta_{3}$. Thus, the total rotation angle $\xi_{\text {all }}$ was given by $\xi_{\text {all }}=\xi_{1}+\xi_{2}+\xi_{3}=\delta_{4}-\delta_{1}$.

It is noted in the simulations that the rotation of oscillation planes is not uniformly distributed along the axial direction, for instance, the rotation angles between adjacent planes $\xi_{1}, \xi_{2}, \xi_{3}$ of $13-3 \mathrm{MT}(L=800 \mathrm{~nm}, \mathrm{m=4})$ were measured as $3.79^{\circ}, 2.58^{\circ}, 3.53^{\circ}$ respectively. The results showed that the rotation become more pronounced at the two ends of the MT. In addition, the total rotation angle $\xi_{\text {all }}$ increases as the half wave number $m$ become larger, for instance, $\xi_{\text {all }}$ of the aforementioned MT is $2.32^{\circ}, 5.35^{\circ}$ and $9.9^{\circ}$ for $m=2,3$ and 4 , respectively. This issue was discussed in more detail in Sec. 4.2.2. Here it should be emphasized that, as shown above, the frequency and thus the energy of the vibration does not change significantly in the presence of the oscillation plane rotation. This suggested that no significant torsion occurred for the vibrating MTs. The rotation of the oscillation plane is merely due to the continuous rotation of the bending axis on the cross-sections when $z$-coordinate increases from 0 to $L$ (MT length) (Fig. 4.2(c)).

To find a possible explanation of the observed phenomenon we restored to the bending theory of EB model [218] as bending occurs for the MT in the transverse vibration. Thus, the vibration frequency is determined by the second moment of inertia $I$ of the MT cross-section. However, as shown in Fig. 4.3(a-c), due to the helical structure of 13-3 MTs the material is not uniformly distributed along the perimeter of the MT cross-section. In particular, the distribution varies with the $S$ index as shown in Fig. 4.3(e) and was also found to change or rotate from cross-section A-A to its adjacent cross-section B-B. It is understood that the frequency is exactly the same everywhere on the MT, suggesting that the value of the inertia $I$ on the individual cross-sections of the MT should be nearly the same. Consequently, the different material distribution on the adjacent cross sections finally lead to the rotation of the bending axis between the two adjacent cross sections to maintain the identical inertia $I$ and accordingly, the same vibration frequency. Thus, 
the helical structure of MT can at least partially explain the physical mechanisms of the $3 \mathrm{D}$ transverse vibration. Here we are very keen to find direct evidence to confirm the existence of the 3D transverse vibration of MTs. Unfortunately, the experimental data are still not available in the literature. On the other hand, it is noted that this vibration mode is similar to the 3D bending reported for cantilevered MTs based on a FE model in [65]. The FE simulations can to some extent support the present study where the 3D transverse vibration was observed.
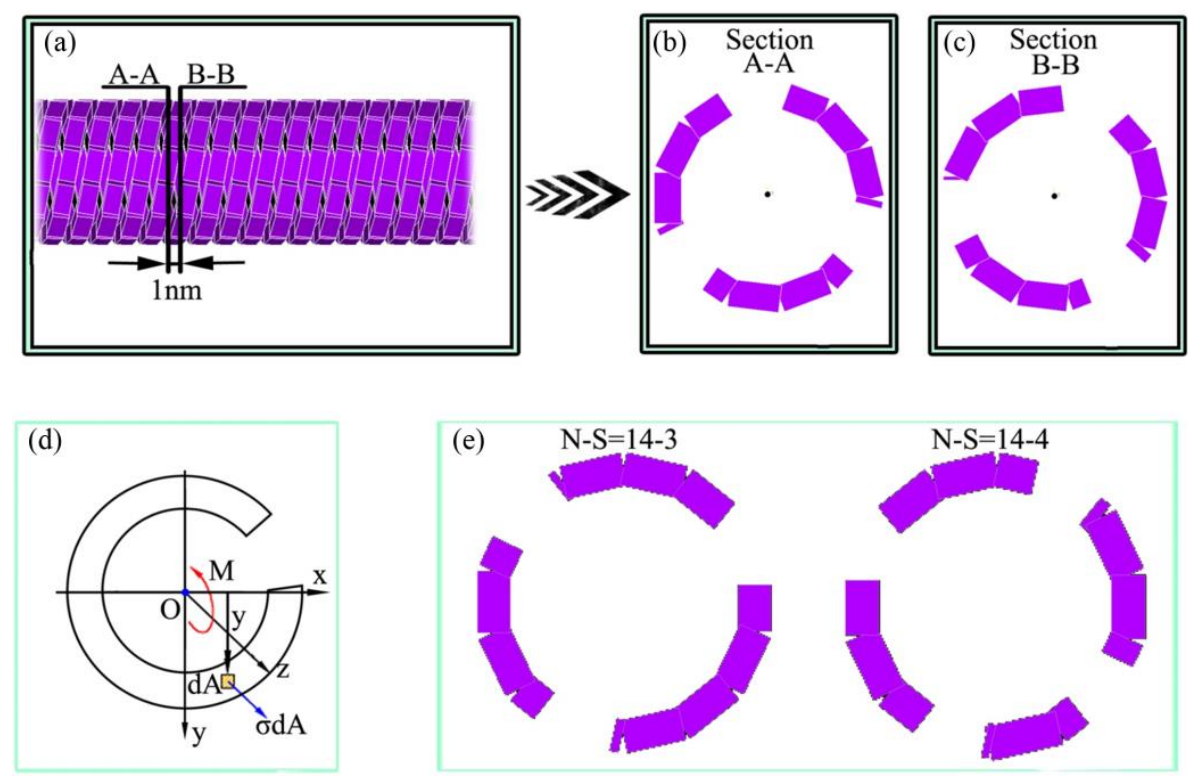

Figure 4.3 The material distribution on the perimeter of MTs with different S.

\subsubsection{Factors influencing the 3D vibration}

The novel 3D transverse vibration mode was achieved in the previous section for 13-3 MTs. In this section, we further examined the effect of the major factors that may exert significant effects on this vibration mode or the rotation angle. The factors can be categorized into two groups including the internal factors, i.e., the structural details of MTs and the external factors, such as length, vibration modes and the boundary conditions.

\subsubsection{Internal factors}

To examine the effect of MT structures 10 MTs with different $N-S$ parameters 
were considered (Table 2.1) and the rotation angle $\xi_{\text {all }}$ defined in Sec. 4.2.1 and shown in Fig. 4.2(c) was calculated for the 4th mode of these sample MTs. The results were shown as a function of helix-start $S$ in Fig. 4.4(a) and PF number $N$ in Fig. 4.4(b), respectively.
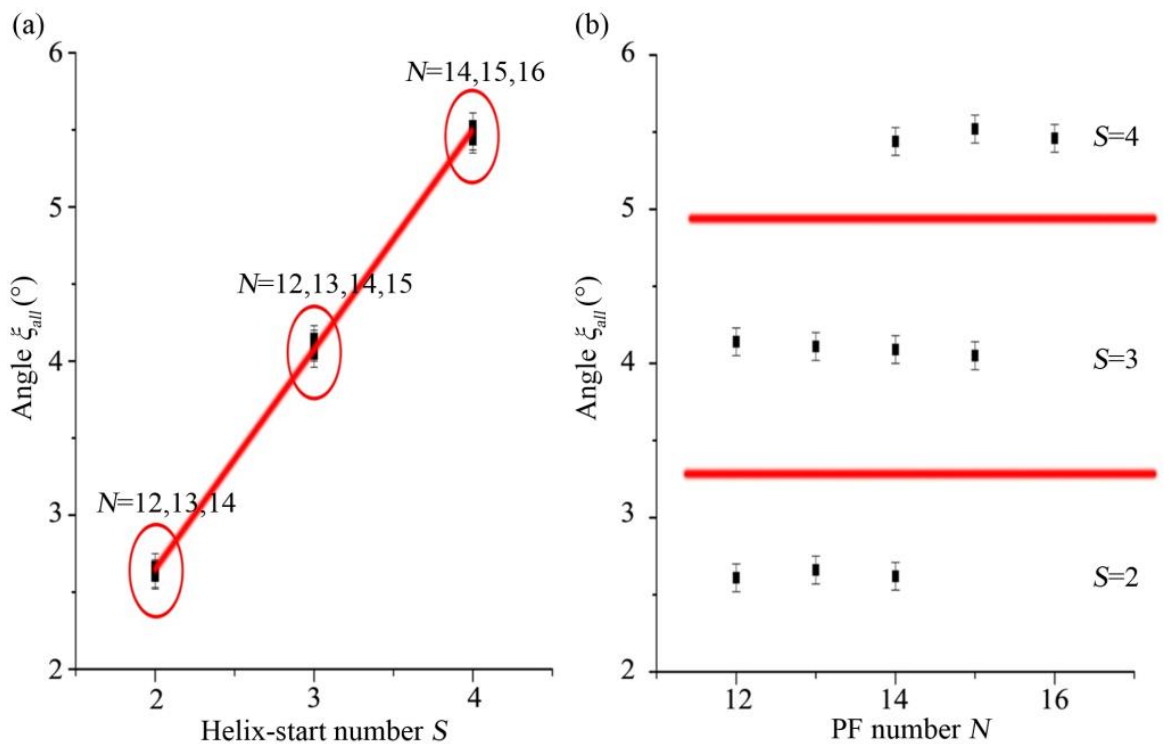

Figure 4.4 Dependence of the rotation angle $\xi_{\text {all }}(L=2000 \mathrm{~nm})$ on (a) the helical start number $\mathrm{S}$ and (b) the number of protofilaments $N$ obtained for the $4^{\text {th }}$ model of the MT vibration.

It is noted in Fig. 4.4(a) that the angle increases linearly with rising $S$. The slope of the curve is around $1.42^{\circ} /$ per unit $S$. Fitting the data in Fig. 4.4(a) yielded the following linear relation between the total rotation angle $\xi_{\text {all }}$ and the helix start number $S$, showing a strong dependency of $\xi_{\text {all }}$ on the helical structures of MTs.

$$
\xi_{\text {all }}=-0.195+1.422 \cdot S \quad(S=1,2,3)
$$

These results provide clear evidence that, as commented in Sec. 4.2.1 the helical structures of MTs should be responsible for the rotation of the oscillation planes or the bending axis of the cross sections shown in Fig. 4.2. On the other hand, it is seen from Fig. 4.4(b) that, for a given $S$ the angle $\xi_{\text {all }}$ remains nearly a constant independent of the PF number $N$. Thus, the PF number $N$ does not exert significant influence on the rotation angle directly. Here it is noted that, for 13MTs with skew angle $\theta_{m}=0$ Eq. 2.1 reduces to $r_{m}=\frac{S a_{m}}{N}$. Thus when $S$ is fixed and $N$ changes, while 
the rotation angle remains constant, the subunit rise $r_{m}$ and also the helical angle $\frac{r_{m}}{\delta x}$ will change substantially. Here $\delta x$ is the PF separation (Fig. 2.1). From these analyses it follows that the rotation of the bending axis is independent of the subunit rise or the helical angle of MTs. It is primarily controlled by the helicity start number $S$ describing the periodic change of structure in the axial or PF direction.

Furthermore, in Fig. 4.5 we calculated the frequency for the MTs considered in Fig. 4.4. The results show that the frequency generally increased with rising $N$ or the diameter of MTs. This $\mathrm{N}$-dependency turns out to be more significant for higher modes with larger $m$. On the contrary, for a given $N$ the frequency of all the modes selected remains unchanged when the helical start number $S$ varying between 2 to 4 . These results are found to be consistent with the results obtained previously in [78].

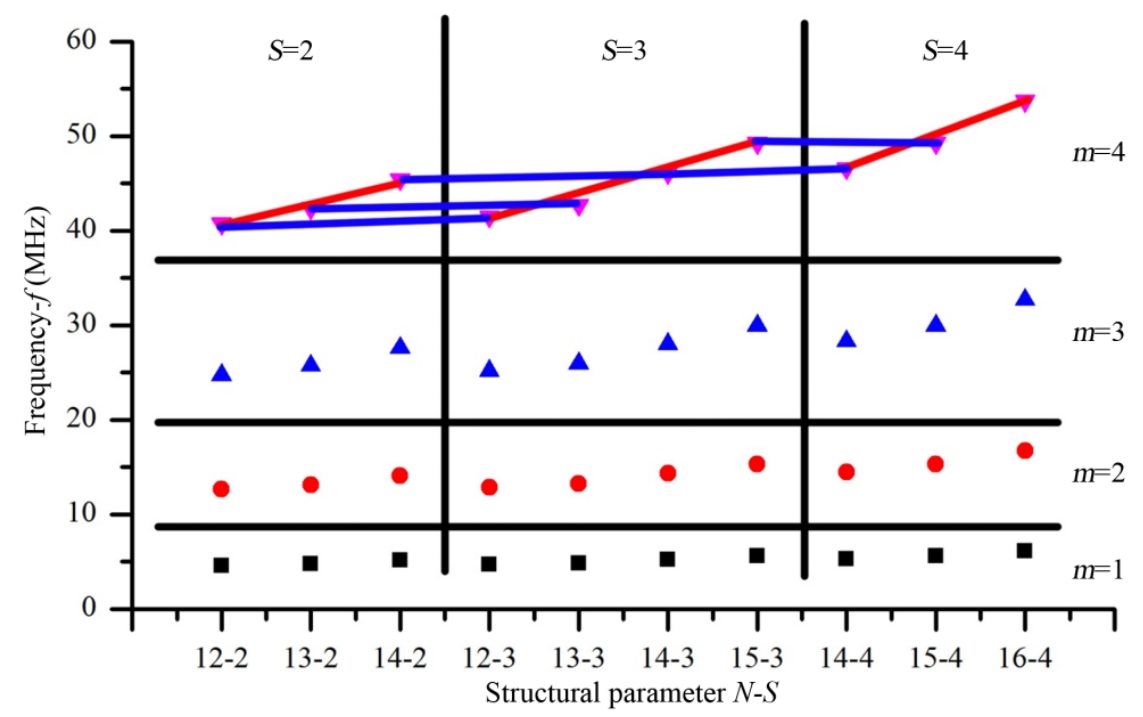

Figure 4.5 The effect of structural details on the frequency of MTs with different $S$ and $N$. Here the half wave number $m$ changes from 1 to 4 .

\subsubsection{External factors}

In addition to the MT structures there also exist some external factors that may affect the rotation angle. These factors may include the contour length (or wavelength) of MTs, the mode number of the vibrations and the boundary conditions 
on the two ends of MTs. It is thus of interest to measure their effects on the rotation angle $\xi_{\text {all }}$ for MTs. To this end we calculated the angle $\xi_{\text {all }}$ of 13-3 MTs with different lengths. The results obtained for the 3 modes with $m=2,3,4$ were plotted in Fig. 4.6 against the contour length of MTs. As shown in the figure, for a given vibration mode the angle $\xi_{\text {all }}$ decreases monotonically with the increasing contour length. The rate of change increases when the contour length becomes shorter. The second order polynomial fitting for $m=2,3,4$ was also given in Fig. 4.6 with R-square of $0.89045,0.98056,0.98496$, respectively.

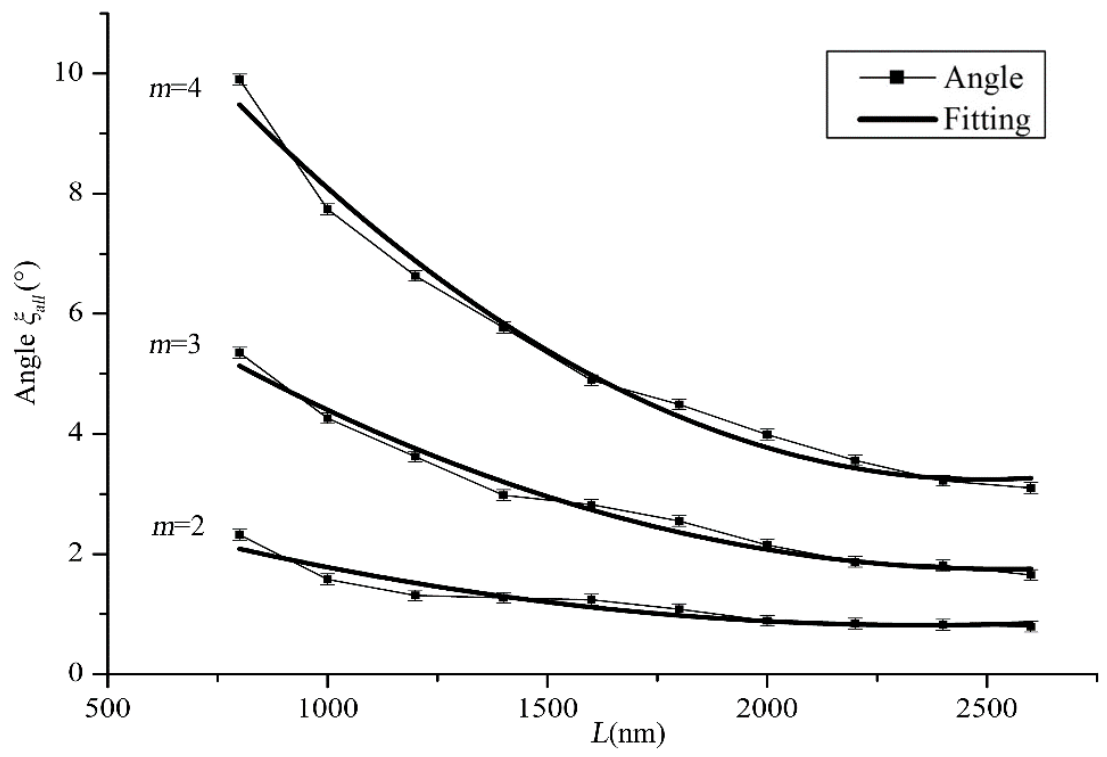

Figure 4.6 The length dependence of the angle $\xi_{\text {all }}$ of standard 13-3 MTs

In addition, Fig. 4.6 indicated that, for a given contour length the rotation angle $\xi_{\text {all }}$ increases with rising mode number $m$. The rate of change in angle $\xi_{\text {all }}$ is found to increase with decreasing contour length. As mentioned above, when the contour length decreases or the mode number $m$ increases the rotation angle $\xi_{\text {all }}$ becomes greater. This can be attributed to the fact that the (half) wave length declines in these two processes. Thus we came to the conclusion that the rotation angle $\xi_{\text {all }}$ is larger for the vibration with a shorter wave length. Further it was seen from Fig. 4.6 that, for the same wave length the total rotation angle $\xi_{\text {all }}$ increases with increasing half wave number $m$. For example, in Fig. 4.6 MT vibrations with $(m, L)=(2,1000 \mathrm{~nm})$, $(m, L)=(3,1500 \mathrm{~nm})$ and $(m, L)=(4,2000 \mathrm{~nm})$ have the same half wave length 
$500 \mathrm{~nm}$ but the corresponding rotation angle $\xi_{\text {all }}$ increases from $1.58^{\circ}$, to $2.96^{\circ}$ and to $3.99^{\circ}$ when $m$ rises from 2 to 3 and to 4 . This shows the relation between the rotation angle $\xi_{\text {all }}$ and the half wave number $m$ when the half wave length was kept constant. It is thus evident that, for a fixed (half) wavelength $\xi_{\text {all }}$ increases with rising $m$ and thus growing contour length. The decrease of $\xi_{\text {all }}$ with rising length observed in Fig. 4.6 is due to the fact that for a given $m$ the (half) wavelength increases with growing contour length. It is thus evident that the key external factors determining the rotation angles are the (half) wavelength and half wave number (or mode number) $m$ rather than the contour length.

Another external factor that may significantly alter the rotation angle of $\xi_{\text {all }}$ is the constrains imposed on the two ends of MTs. To examine the boundary condition effects, we considered the transverse vibration of the 13-3 MTs with the same contour length $1200 \mathrm{~nm}$ but two different boundaries conditions, i.e., (1) the two ends are fixed (i.e., fixed-fixed condition) and (2) one end is fixed and the other free (i.e., cantilever condition). The shapes of mode $4(m=4)$ were given in Fig. 4.7 for the MTs and the rotation angle $\xi_{\text {all }}$ for the MTs were measured as $6.63 \pm 0.09^{\circ}$ and $6.15 \pm 0.09^{\circ}$, respectively, when fixed-fixed and cantilever boundary conditions were considered. When mode number decreases from 4 to 3 and 2, the angle associated with fixed-fixed ends decreases from $6.63 \pm 0.09^{\circ}$ to $3.62 \pm 0.09^{\circ}$ and $1.31 \pm 0.09^{\circ}$. Those obtained for the cantilever boundary condition declines from $6.15 \pm 0.09^{\circ}$ to $3.16 \pm 0.09^{\circ}$ and to $1.19 \pm 0.09^{\circ}$. These results clearly show that the fixed-fixed ends lead to the rotation angles significantly larger than those associated with the cantilever boundary condition. In other words, the rotation of the bending axis of the cross-section would become more substantial when more constrains were enforced on the two ends of MTs.

As shown above, the 3D transverse vibration mode was achieved as a result of the helix structures of MTs. This shows clear evidence that the unique structures of MTs lead to a deformation pattern significantly different from that of an elastic beam. 
The new finding is a step forward in gaining an in-depth understanding of the mechanisms via which the MT structures deform in a unique way to fulfil their functions in various physiological processes. The vibration mode-structure relation may also provide useful guidance for the development of the MT-based biomimetic materials whose performance depends heavily on its transverse vibration. Herein, another thing worth mentioning is the damping effect of cytosol, which has to be taken into consideration for MTs in vivo. This issue has not been discussed here as this section is focused on the structure-property relation of individual MTs, e.g., MTs in vitro.

(a)

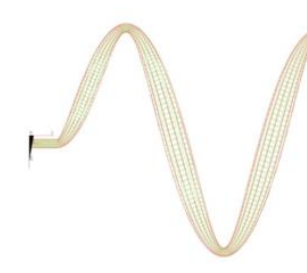

(c) (b)

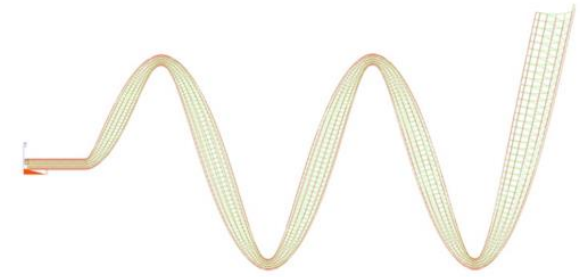

(d)

Figure 4.7 Mode shapes of a vibrating MT with (a) fixed-fixed ends and (b) fixed-free ends; The YOX projections of the mode shapes are shown in (c) and (d), respectively.

\subsection{Tension induced bending in $\mathbf{F}$-actin}

Based on the MSM and the obtained force constants, we performed tensile tests for F-actin. It is interesting to see in the tensile test of F-actin (Fig. 4.8) that for a given tensile force $F_{1}$, the stretching of F-actin is always accompanied with a transverse deflection $w$, which increases with the rising contour length $L$. In addition, for a given $L$ the deflection at the angle of $166.15^{\circ}$ was found to be larger than the 
deflection associated with the angle of $167.14^{\circ}$.

Moreover, the tension-induced transverse deflection gradually increases with the increasing tensile force applied or the rising elongation of the F-actin (Fig. 4.8). Similar transverse deflection was also observed for F-actin when a compressive force is applied. To capture the underlying physics of the observation, we used polynomial functions to fit the $L-w$ (transverse deflection) relation obtained in the MSM simulations (Fig. 4.8). It was found that the $2^{\text {nd }}$ order polynomial was the best fit to the simulation results, which is in agreement with the bending theory of beams [218] where the deflection $w$ of beams subject to a moment $M$ is proportional to $L^{2}$. This infers that the bending of F-actin should be a result of a bending moment generated by the tensile force applied. Indeed, more detailed study showed that due to the helical structure of F-actins, the central axis of F-actin does not coincide with the

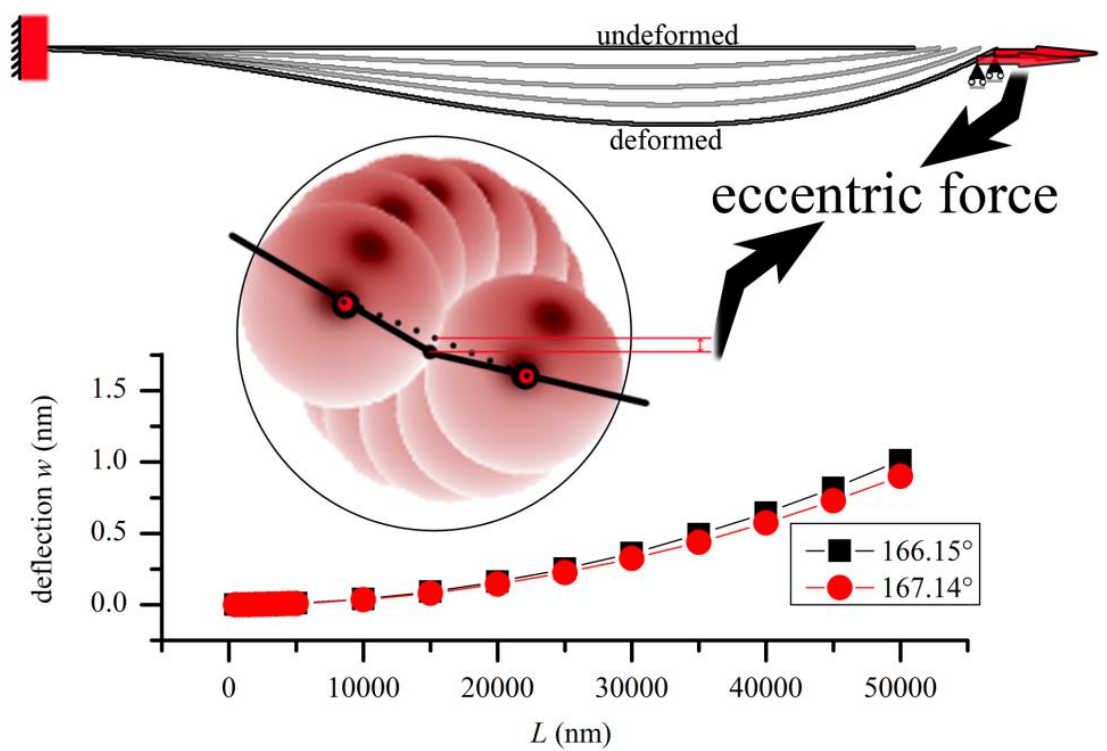

Figure 4.8 The tension induced bending of F-actin

position of the resultant force on the cross section when the tensile forces are applied through the centres of the two adjacent monomers (see Fig. 4.8). In other words, there exists a resultant eccentric force $F_{\text {eccentric }}$ which can thus generate a bending moment $M=F_{\text {eccentric }} \times \Delta R$ relative to the central axis of the F-actin. Here $\Delta R$ is the distance between the eccentric force and the central axis. Consequently, the observed transverse deflection can be primarily attributed to this additional moment arising 
from the eccentric resultant force or the helical structure of F-actin. Moreover, a higher angular separation $167.14^{\circ}$ results in a smaller $\Delta R$, and thus, a lower bending moment and a smaller transverse deflection. Also, with the same bending moment the deflection of F-actin naturally grows with the increasing contour length. The eccentric force found in the F-actin thus offers a possible physical explanation for the tension-induced bending and the length and angle dependency of the bending deflection observed in the MSM simulations.

Furthermore, the boundary condition is found to be an important factor that controls the tension-induced bending. As mentioned in the section of model development (Sec. 2.3.4), during the tensile test, the force was applied on the 2 monomers which were pinned on axial rollers. As illustrated on the top of Fig. 4.9, if the monomers were fixed on rollers in the tensile test, the deflection becomes negligible compared to the one associated with the pinned condition. Although the actual end condition of in vivo filaments are not clear [247], the actual end condition could be reasonably assumed to be an intermediate state between the fixed end and pinned end. Thus, the bending induced by the tensile force may occur for the in vivo F-actin and can provide a possible explanation for the curved configuration observed for most F-actins [11, 248].

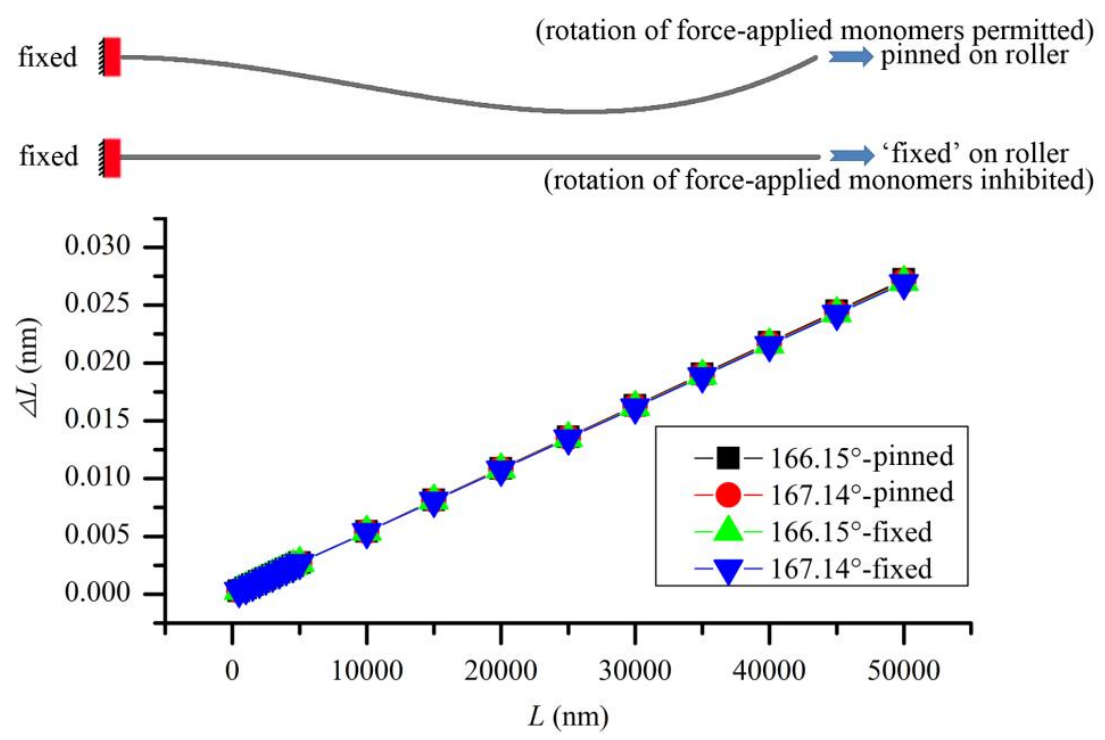

Figure 4.9 Boundary condition effect on F-actin stretching 


\subsection{Summary}

A unique transverse vibration of helical MTs was achieved via a recently developed MSM model. In sharp contrast to the vibration of classical beams, the bending axis of the cross-section of vibrating MTs rotates throughout the length of the MTs. As a result, the adjacent half-waves vibrate approximately in two different oscillation planes, showing a 3D transverse vibration for MTs. Nevertheless, no real torsion occurs for the MTs and thus the frequency remains nearly the same in the absence or presence of the bending axis rotation.

In addition, the rotation angle was found to increase almost linearly with the increasing helical start number $S$ but remains independent of the PF number $N$. These indicate that the helical structures of MTs are responsible for the 3D transverse vibration of MTs, and the rotation angle of the bending axis is primarily determined by the periodic arrangement of protein monomers in the axial direction. The helical angle, however, does not play a significant role in determining the rotation angle of the bending axis on MT cross sections.

Furthermore, the wavelength of vibration was identified as a characteristic length that can significantly change the rotation angle of the bending axis. The angle was found to rise with the decreasing wavelength or the growing wavenumber (for a given contour length). Additionally, the rotation angle can be further raised by imposing more constraints on MT ends.

Subsequently, the tension-induced bending was studied for F-actins as a result of their helical structure. The MSM simulations also show that the helical structure of F-actin leads to a resultant eccentric force and thus, a resulted bending moment on the cross-section of the F-actin when an axial tension/compression is applied. For a given axial load the induced bending deflection of F-actin increases substantially with the rising contour length but decreases slightly with growing rotation angle. This study provides a possible physical origin for the curved F-actins experimentally observed. 


\section{Chapter 5 Subcellular environment effect on the filament mechanics}

\subsection{Introduction}

As MTs are subjected to compression in cells, buckling occurs for MTs [249], which has attracted considerable attention from the community of cell mechanics [77]. The in vitro buckling was first studied experimentally to quantify the flexural rigidity of MTs [161-163, 250]. The elastic beam models were employed to organize the experimental data $[161,162,250,251]$ and provide an insight into the MT buckling [249]. The beam-like buckling was also reported for MTs in [63, 64]. In 2006, an orthotropic shell model was proposed for MTs [59], and used in the analyses of MT buckling [252, 253].

Here, one of the observations is that MTs in vivo (with subcellular environment effect) possess a critical buckling force $\left(F_{c r}\right)$ higher than those in vitro. Attempts were then made to understand this in terms of the subcellular environment effects, where cytoplasm of cells was simplified as an elastic media [64, 254]. Years later, a one-dimension (1D) FE model was developed [247] where the CSK components around MTs were treated as discrete cross-linkers. This work provides the guidance to examine the role of the discrete cross-linkers in MT buckling $[255,256]$ and offers a pathway to more realistic delineation of MT buckling in vivo. Motivated by this study, the Sec. 5.3 is devoted to further studying localized MT buckling by using a 3D MSM model. The major issue examined is the effect of the spatial, random and inhomogeneous distributions of the proteinaceous linkers.

In addition, filopodia are thin, actin-rich plasma-membrane protrusions, which function as antennae for cells to probe their environment [257]. The protrusive bundles in filopodia consist of F-actins cross-linked by ABPs. The growth of these F-actins generates force for protrusion of the leading edge during cell motility [28]. In the study of the filopodial protrusion, the major issues are (1) the upper limit of 
the force the F-actins can generate and (2) the underlying physical mechanisms based on which the critical value can be determined. The critical buckling load of the actin bundles may serve as a criterion in evaluating the stall force on the actin bundle [51, 258] and the maximum length of the filopodial protrusion. In the meantime, a ratcheting model [27] considering a different mechanism was also proposed to predict the maximum force on the tip of the F-actins. It was reported in Ref. [258] that these two mechanisms may coexist and one of them can be predominant depending on the monomer concentration in cytosol. In Section 5.4, we considered the condition at which the buckling is predominant mechanism in controlling the stall force on the filopodial actin bundles. The MSM model developed for the filopodial actin bundles was used in the buckling analyses, which is able to account for the structure-buckling response relation of the F-actin bundles. The model was utilized to explore the buckling behaviour and obtain the critical buckling load for the filopodial F-actins. Thus, the influence of the ABPs was examined in forming the filopodia protrusion.

It is worth mentioning that the MSM simulations in the present chapter were carried out with a desktop computer with 4 cores.

\subsection{Model development}

\subsubsection{The details of the MSM model for buckling simulation}

In a practical structure analysis, the global stiffness matrix of the frame includes global elastic stiffness matrix $\boldsymbol{K}_{\boldsymbol{e}}$ and global geometric stiffness matrix $\boldsymbol{K}_{\boldsymbol{g}} . \boldsymbol{K}_{\boldsymbol{g}}$ reflects the change of stiffness of structure under deformation and has a direct relationship with the applied force. If $\boldsymbol{K}_{\boldsymbol{e}}$ is known, $\boldsymbol{K}_{\boldsymbol{g}}$ could be obtained by following conventional procedures in the structural analysis [259]. The external load $N$ is assumed to be

$$
N=\lambda N^{\prime}
$$

where $\lambda$ denotes a constant multiplier and $N^{\prime}$ is the relative magnitudes of the applied 
force. Then the global geometric stiffness matrix $\boldsymbol{K}_{\mathrm{g}}$ could be obtained as follows:

$$
\boldsymbol{K}_{\boldsymbol{g}}=\lambda \boldsymbol{K}_{\boldsymbol{g}}^{\prime}
$$

where $\boldsymbol{K}_{\boldsymbol{g}}^{\prime}$ represents the geometric stiffness matrix for the applied load $N^{\prime}$. This equation is based on the assumption that the geometric stiffness matrix $\left(\boldsymbol{K}_{\boldsymbol{g}}^{\prime}\right)$ is proportional to the internal forces at the onset of the loading step $\left(N^{\prime}\right)$. The global elastic stiffness matrix can be considered as unchanged for a wide range of displacement $\boldsymbol{u}$. Thus, there is the relation [259]:

$$
\left(\boldsymbol{K}_{\boldsymbol{e}}+\lambda \boldsymbol{K}_{\boldsymbol{g}}^{\prime}\right) \boldsymbol{u}=\lambda N^{\prime}
$$

At the bifurcation point, the stiffness of the structure vanishes. Therefore, the determinant of the structure stiffness matrix must be zero, hence

$$
\left|\boldsymbol{K}_{e}+\lambda \boldsymbol{K}_{g}^{\prime}\right| \boldsymbol{u}=0
$$

By solving the eigenvalue Eq. 5.4, the lowest value of $\lambda$, i.e. $\lambda_{\mathrm{cr}}$, could be obtained. The critical buckling load is given by

$$
N_{c r}=\lambda_{c r} N_{c r}^{\prime}
$$

Since the external load is not specified in the above deviation, the compression, bending, even torsion and other types of loads could be applied.

\subsubsection{The model of the cross-linkers system supporting MTs}

In this study, we considered 13-3 $(N=13 \quad S=3)$ MT of the most common configuration in in vivo situation $[49,54,260]$. The observed MT buckling behaviours of MTs in cell are different from the ideal buckling mode of an individual MT (Fig 5.1(a)). Therefore, the effect of protein linkers around MTs has to be considered and the structure of the cross-linkers system supporting MTs laterally was depicted in Fig. 5.1. All cross-linkers were modelled as springs with one end attached to the MT and the other end fixed to the surrounding media. The spring constant $k_{s c}$ was taken as $39 \mathrm{pN} / \mathrm{nm}$ [106] and the spatial density of the linkers was defined by the distance $L_{d}=100 \mathrm{~nm}$ between the adjacent linkers for MT of length $L=5 \mu \mathrm{m}$. As the cross-linkers took axial tensile load with negligible compression $[68,261]$, the spring 
modulus was set to be zero in compression. Herein, one MT end was clamped and the other was constrained by a roller. An axial compressive force was then applied to this roller end.

(a)

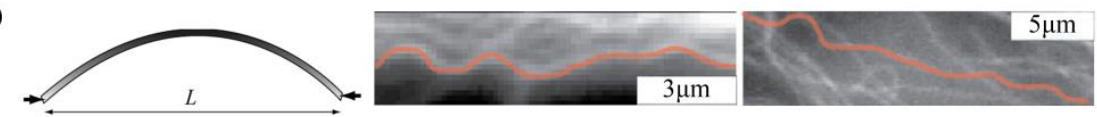

(b)

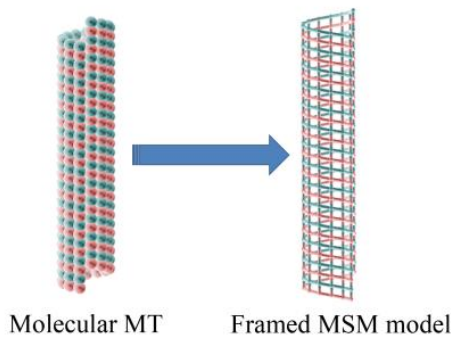

Randomly distributed crosslinkers

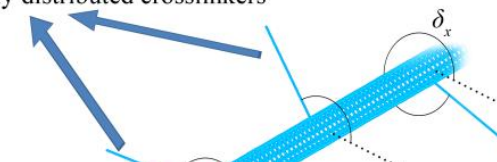

(c)

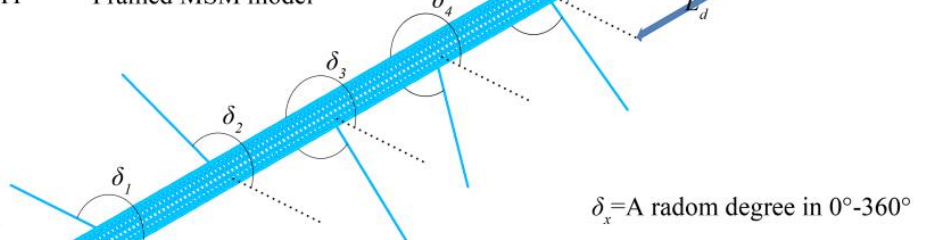

Figure 5.1 (a) The ideal individual MT buckling mode and the MT buckling observations in experimental study [249], (b) Structural representation of an MT and the MSM model, and (c) the structure of the cross-linker system supporting MTs

As shown in Fig. 5.1, a reference direction was picked up in the radial direction of an MT (dotted line) and the orientation of a radial linker was specified by measuring its angle $\vartheta_{\text {rand }}$ relative to the reference direction. The 3D cross-linkers were then achieved by generating the linkers with $\vartheta_{\text {rand }}$ randomly selected between $0^{\circ}$ and $360^{\circ}$ (anticlockwise positive). It is thus should be pointed out that, for 'MT-cross linker' systems considered in Secs.5.3.1 and 5.3.2, $L_{d}, L$ and $k_{s c}$ remain the same but the randomly generated distribution of linker orientation varied from one system to another. The non-uniform linkers [256] were also considered for MTs of length 10 $\mu \mathrm{m}$ in Sec.5.3.3 where a part of the MT was supported by the dense-linkers $\left(L_{d}\right.$ $=25 \mathrm{~nm})$ and the rest was occupied by the low-density linkers $\left(L_{d}=200 \mathrm{~nm}\right)$. The angle between adjacent linkers is fixed at $60^{\circ}$.

Based on the MSM model, the length-dependent critical buckling load $F_{c r}$ was calculated for individual MTs and shown in Fig. 5.2 in comparison with the 
experimental data obtained for in vitro MTs [163]. The excellent agreement has been achieved.

Unfortunately, due to the uncertainty in in vivo condition, such a direct comparison cannot be made for the MT buckling supported by the cross-linkers. In spite of this, the MSM model predicted $F_{c r}$ of the order of $100 \mathrm{pN}$ is comparable to

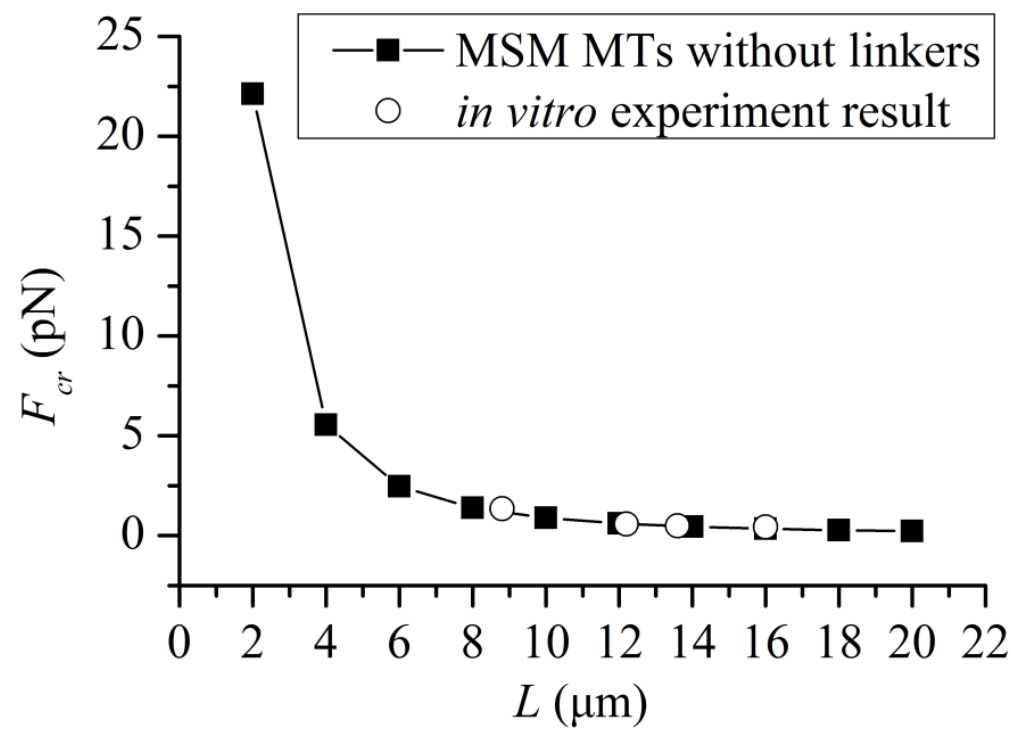

Figure 5.2 The length-dependent $F_{c r}$ calculated for individual MTs in comparison with the experimental data

Table 5.1 Comparison to the cross-linked 1D-FE model

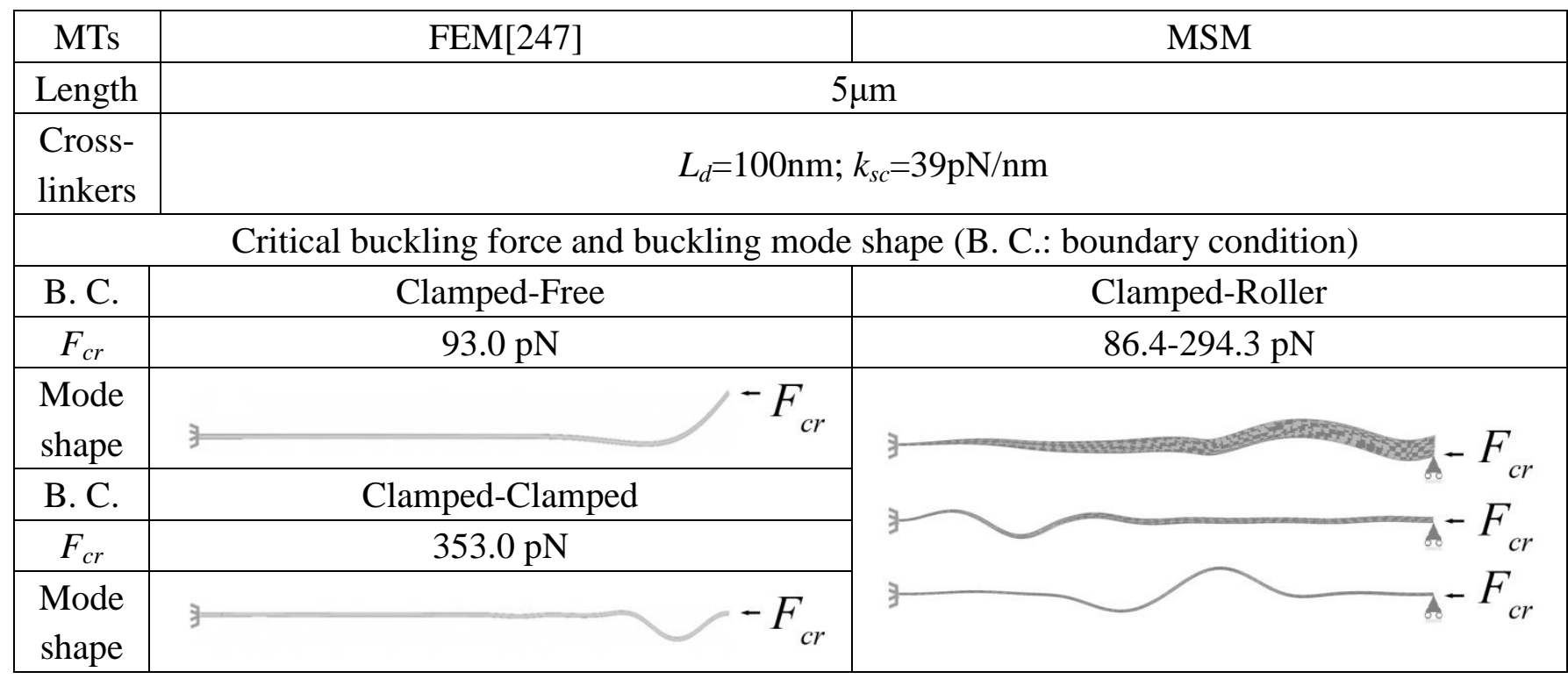

the experimental data [249] and theoretical results [64, 254]. A more detailed comparison was made in Table 5.1 between the MSM model and the 1D-FE model 
accounting for the cross-linker effect [247]. The MT length, constant density and elastic modulus of the linkers considered for the two models are exactly the same. Here, the MSM simulations considered the cross-linkers of 10 randomly generated orientation distributions. $F_{c r}$ achieved for the MT with the clamped-roller ends fell in the range of $[86.4 \mathrm{pN}, 294.3 \mathrm{pN}]$, quite close to $[93 \mathrm{pN}, 353 \mathrm{pN}]$ given by the FE model for Clamped-Free and Clamped-Clamped ends. The localized mode shapes predicted by both models (Table 5.1) resemble those observed experimentally in [249]. In addition, different from the 1D FE model the 3D MSM model is able to capture the unique buckling mode observed in [249], i.e., the 'localized buckling' occurred at different places of MTs. The MT buckling with radial expansion was also achieved by the 3D MSM in Table 5.1. Such radial buckling modes were not observed in experiments probably due to its very small deformation.

\subsubsection{The Model of F-actin supported by the ABPs}

A filopodia F-actin bundle consists of a number of parallel $\mathrm{F}$-actins that are bounded by the ABPs. It is thus of importance to develop a modelling technique for F-actin by considering the stiffening effect of ABPs. It was noted that different ABPs were used to construct F-actin bundles [262-267]. The present study however is focused on the F-actin bundle in filopodial protrusion. Thus ABP considered here is fascin which is prevalent in filopodia [262]. Herein, the ABPs were modelled as linear springs which are able to withstand axial tension but unable to resist compression $[68,261]$. The length of ABPs was taken as $9 \mathrm{~nm}$ and their longitudinal repeat along an F-actin was $36 \mathrm{~nm}$ [268] (Fig. 5.3(a)). Specifically, the constituent F-actins of a filopodial actin bundle are bounded to form a hexagonal lattice [269]. Accordingly, ABPs was assumed to distribute uniformly in circumferential direction as shown in Fig. 5.3(b). The angle between adjacent ABPs was set to be $60^{\circ}$ based on the hexagonal lattice [269]. One end of the $\left(166.15^{\circ}\right)$ F-actin was fixed and the other end was allowed to slide only along the axial direction. The external force was then 
applied to the two monomers of the end that is able to move. The extensional stiffness of ABPs probed by single molecule unfolding experiments ranges from 1 $\mathrm{pN} / \mathrm{nm}$ to $1000 \mathrm{pN} / \mathrm{nm}$ [262]. Fitting the present model to the experimental data enables us to determine the equivalent spring constants required in the spring model for ABPs. A similar modelling technique was effectively used in studying the critical buckling force of in vivo MTs [247].

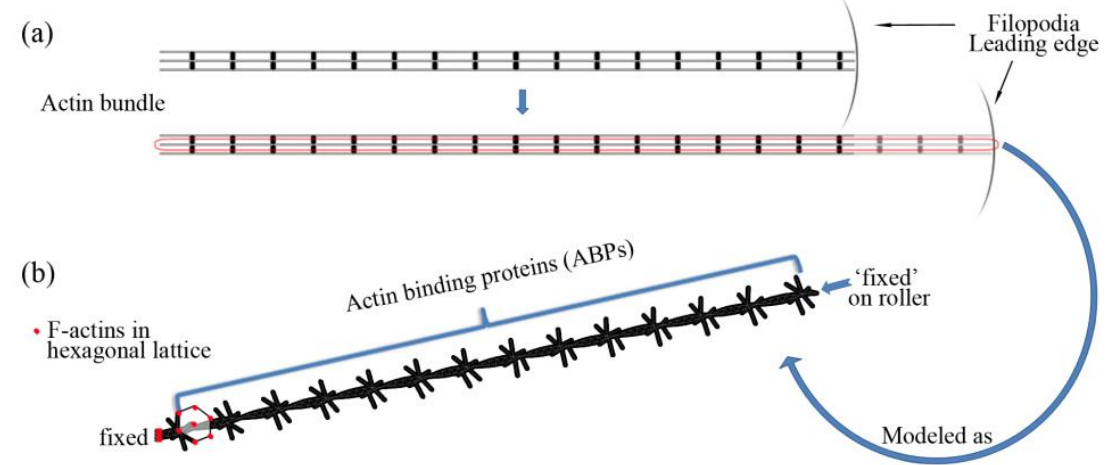

Figure 5.3 (a) F-actin bundle in the protrusion of the leading edge in motile cells, and (b) the model of F-actin supported by ABPs in filopodia protrusion.

\subsection{Effects of the cross-linkers on the buckling of MTs in cells}

In this section, the cross-linker model and the MSM model were employed to study the buckling of in vivo MTs, and examined the influence of the cross-linkers on MT buckling.

\subsubsection{Transition due to the addition of the 3D-linkers}

Herein, ten randomly generated cross-linker distributions were considered for the buckling of MT. The buckling modes of the MT-linker systems with the highest and lowest critical buckling load $F_{c r}$, were presented in Fig. 5.4. It was noticed that the randomly generated linkers lead to diverse buckling modes associated with significant variation in $F_{c r}$ values. It is thus of interest to examine the effect of the spatial cross-linkers. 
To this end, we first considered 2D-linkers, which were parallelly aligned and distributed uniformly with a constant spatial density (Fig. 5.5(a)). Subsequently, the 2D-linkers close to the clamped end were replaced by the 3D-linkers where the axial density remained unchanged but the radial orientations were randomly selected

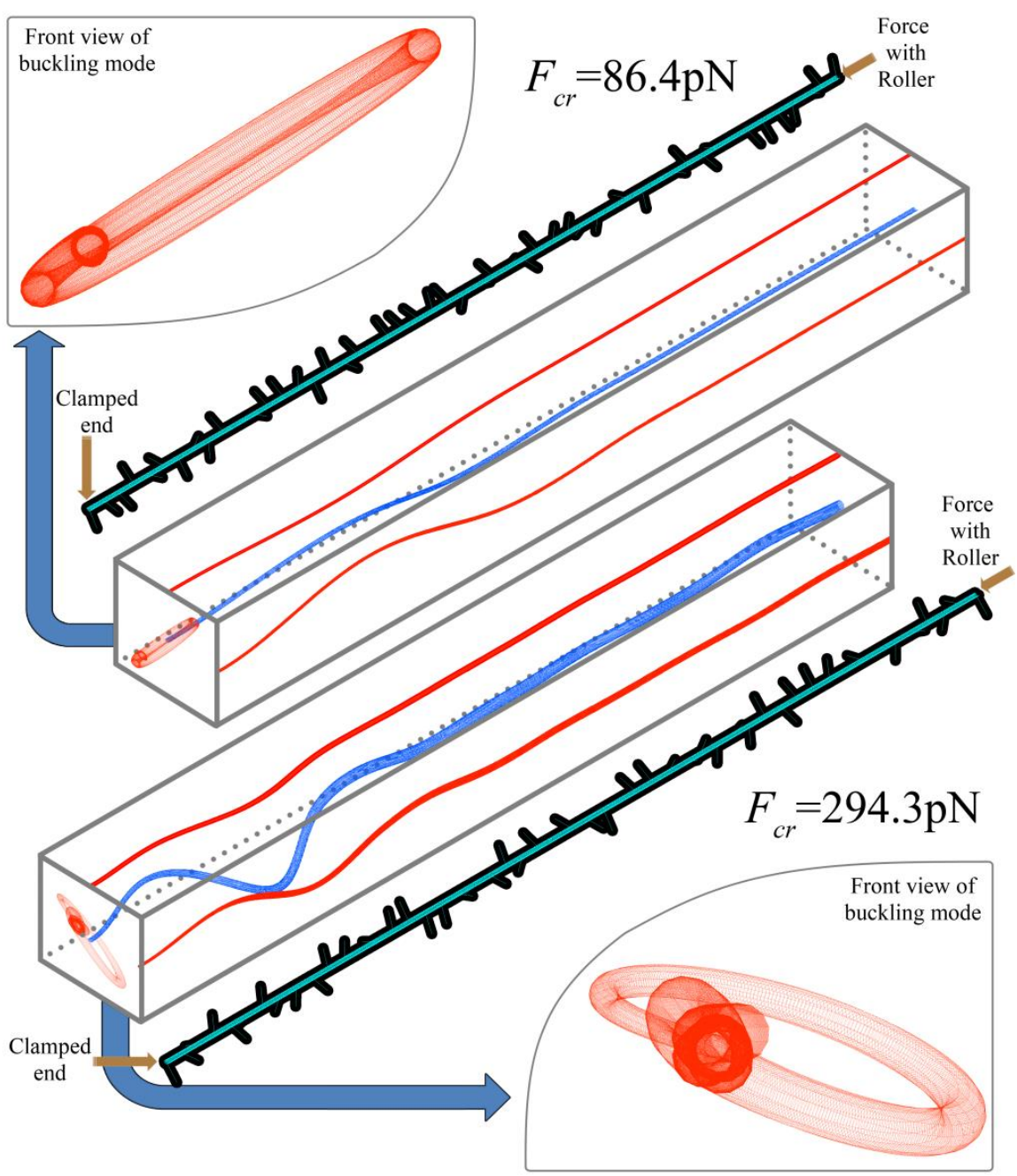

Figure 5.4 The 3D buckling modes of the selected MT-linker systems with randomly generated cross-linkers.

between 0 and $360^{\circ}$ (Fig. 5.5(b)). As shown in Fig. 5.5, the 2D-linkers led to the planar bulking with one large half wave and the relatively low critical load $F_{c r}$ (Fig. 5.5(a)). Addition of the 3D-linkers led to enhanced critical buckling load $F_{c r}$. When $\alpha$ (defined as the length covered by the 3D-linkers to MT length ratio) is not more than $30 \%$, the buckled MTs still deform in the same plane (Fig. 5.5(c)) and $F_{c r}$ increases gradually with the rising amount of the 3D linkers. Once $\alpha$ was raised to $40 \%$, the 2D 
beam-like buckling transforms into 3D shell-like buckling, where radial expansion occurred and the central line of the shell become a 3D curved line (Fig. 5.5(c)). Associated with this change is the sudden growth of the buckling load $F_{c r}$ (from the order of $10 p N$ to $100 p N$ ) by one order of magnitude (Fig. 5.5(b)). Further raising $\alpha$ above $40 \%, F_{c r}$ of the $3 \mathrm{D}$ modes again increases progressively. These results given by the MSM model demonstrate the importance of the radial orientation of the cross-linkers in controlling the buckling behaviour of MTs in cells.

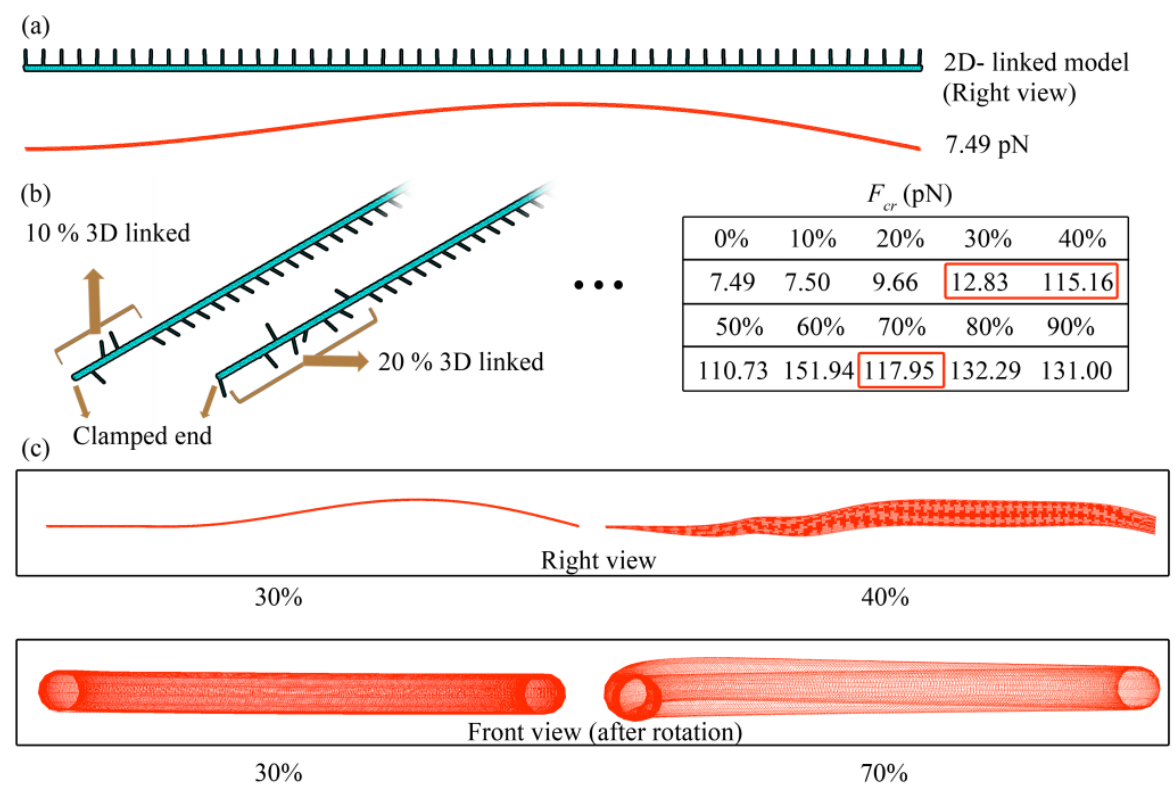

Figure 5.5 The effect of the spatial linker orientations obtained by considering a 2D-linker system and the systems with increasing amount of the 3D-linkers.

\subsubsection{Spatial distribution of the 3D-linkers}

It is noted in our initial study that different critical buckling loads were achieved for the 'MT-3D cross-linker' systems where MT geometry, boundary conditions and the density of the cross-linkers were exactly the same. Obviously, the scattering of the obtained critical buckling load is due to the diverse spatial distributions generated randomly for the 3D-linkers. Thus, a more detailed study was conducted here to further examine the effect of the spatial distributions of the 3D cross-linkers. In doing this, we defined so-called ' $n$-repeat' cross-linkers which comprise groups of 2D 
cross-linkers (Fig. 5.6). The number of the cross-linkers $n$ remains the same for individual groups and the angle between adjacent two groups is $120^{\circ}$ (Fig. 5.6).

The critical buckling load $F_{c r}$ was then calculated in Fig. 5.6 for various MT-3D linker systems where the density $L_{d}$ varied from $25 \mathrm{~nm}$ to $200 \mathrm{~nm}$ and the number of $n$-repeat grow from 1 to 25 . To demonstrate the physical meaning of $n$-repeat let us consider a system with MT length $175 \mathrm{~nm}$ and $L_{d}=25 \mathrm{~nm}$. In this case, $n$-repeat $=1$ gives pure $3 \mathrm{D}$ linkers where the angle between the adjacent linkers is $120^{\circ}$. Increasing $n$-repeat to 4 , we have the $3 \mathrm{D}$ linkers comprising 2 groups of $2 \mathrm{D}$ linkers (each has 4 linkers) and the angle between the two groups is again $120^{\circ}$. Further raising $n$-repeat to 8 leads to $2 \mathrm{D}$ linkers. Thus, smaller $n$-repeat leads to the $3 \mathrm{D}$ linkers with more uniformly distributed orientation in circumferential direction.

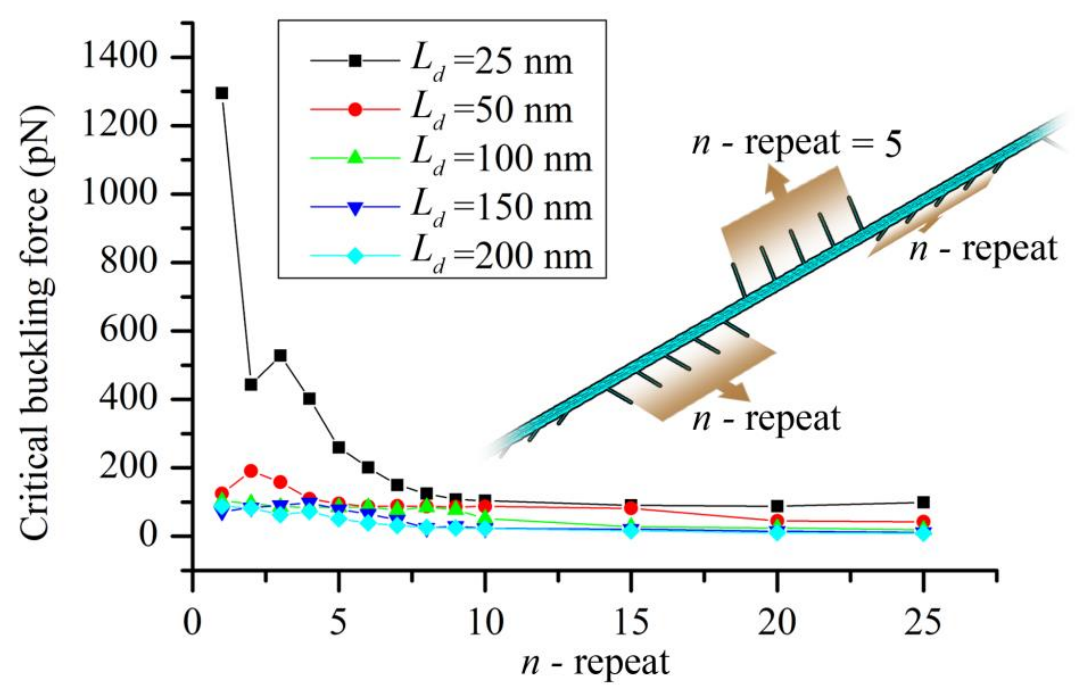

Figure 5.6 The effect of the spatial linker distributions on the critical buckling load obtained by considering the 'n-repeat' linkers

It was found in Fig. 5.6 that for the MT-3D linker systems considered, the highest $F_{c r}$ was obtained at $n$-repeat $=1$, i.e., the MT is supported by the 3D-linkers with uniform distribution of the radial orientation. After this, $F_{c r}$ generally decreases with rising $n$-repeat. This observation indicated that less homogeneous distribution of the linker orientation usually down shifts the critical buckling load. This finding can shed some lights on the varying critical buckling loads achieved for the MT-cross linker systems with the same length and boundary conditions of MT, and the 
identical axial density of the cross-linkers. Furthermore, it was seen from Fig. 5.6 that the effect of the cross-linker orientation turns out to be more significant for the MT-3D linker systems with the lower $L_{d}$ and smaller $n$-repeat. In addition, some exceptions were also found in Fig. 5.6 where at $L_{d}=25 \mathrm{~nm} F_{c r}$ associated with $n$-repeat $=3$ is even higher than that associated with $n$-repeat $=2$. The result suggested that other factors may also exert influence on MT buckling. For the MTs with 2D-linkers or individual MTs, it is well-known that $F_{c r}$ decreases with rising length. This common sense however may not be true for MTs supported by the 3D-linkers due to the effect of their spatial orientation.

\subsubsection{Effect of the local dense 3D-linkers}

The local dense-linkers are found in in vivo condition. A typical example is the dense actin network in the lamellipodia of cells [256]. To examine the effect of the local dense-linkers on MT buckling we considered the MT-linker systems with various sizes and locations of the dense-linkers having $L_{d}=25 \mathrm{~nm}$. The rest of the MT was supported by the low-density linkers with $L_{d}=200 \mathrm{~nm}$. The buckling modes and the associated buckling load were shown in Fig. 5.7 with the dense-linkers regions represented by the coarse lines. Here, buckling preferably occurrs within the low-density linker regions showing the localized features of MT buckling. Such localized buckling close to the force applied tip (right side) was also achieved by the 1D-FE model [247]. In the present simulations, buckling was also found in different places of MTs depending on the location of dense-linkers. These results agreed with the experimental observation and showed that experimentally observed '-deflected-straight-deflected-' MT buckling can be attributed to the local density variations of the surrounding cytoskeletal network [249].

Furthermore, as disclosed in Fig. 5.7(a-h), $F_{c r}$ remains nearly constant when the distance was fixed between the right end of the dense-linkers and the loaded end of the MT (right side), although the length covered by the dense-linkers was varied by 
up to 3 times. During the calculations, the average density of the linkers was also changed substantially. The results thus suggested that the average linker density is not the key factor in controlling the buckling response in the cases considered. In contrast, the position of the right end of the dense-linkers or the length of the lower-density linkers between the local dense-linker and the loaded end of MTs (e.g., MT end close to the cell membrane) play an essential role in determining $F_{c r}$ of MTs in cells. Despite a few exceptions, $F_{c r}$ exhibits a general trend to decrease from $133 \mathrm{pN}$ to $51 \mathrm{pN}$ when the right end of the dense-linkers were moved towards the left, and the length of the low-density linkers close to the force applied end of MTs was increased by a factor of 6 (Fig. 5.7(a-f)). Specifically, in most cases the buckling of MTs is localized to the aforementioned region of the low-density linkers.

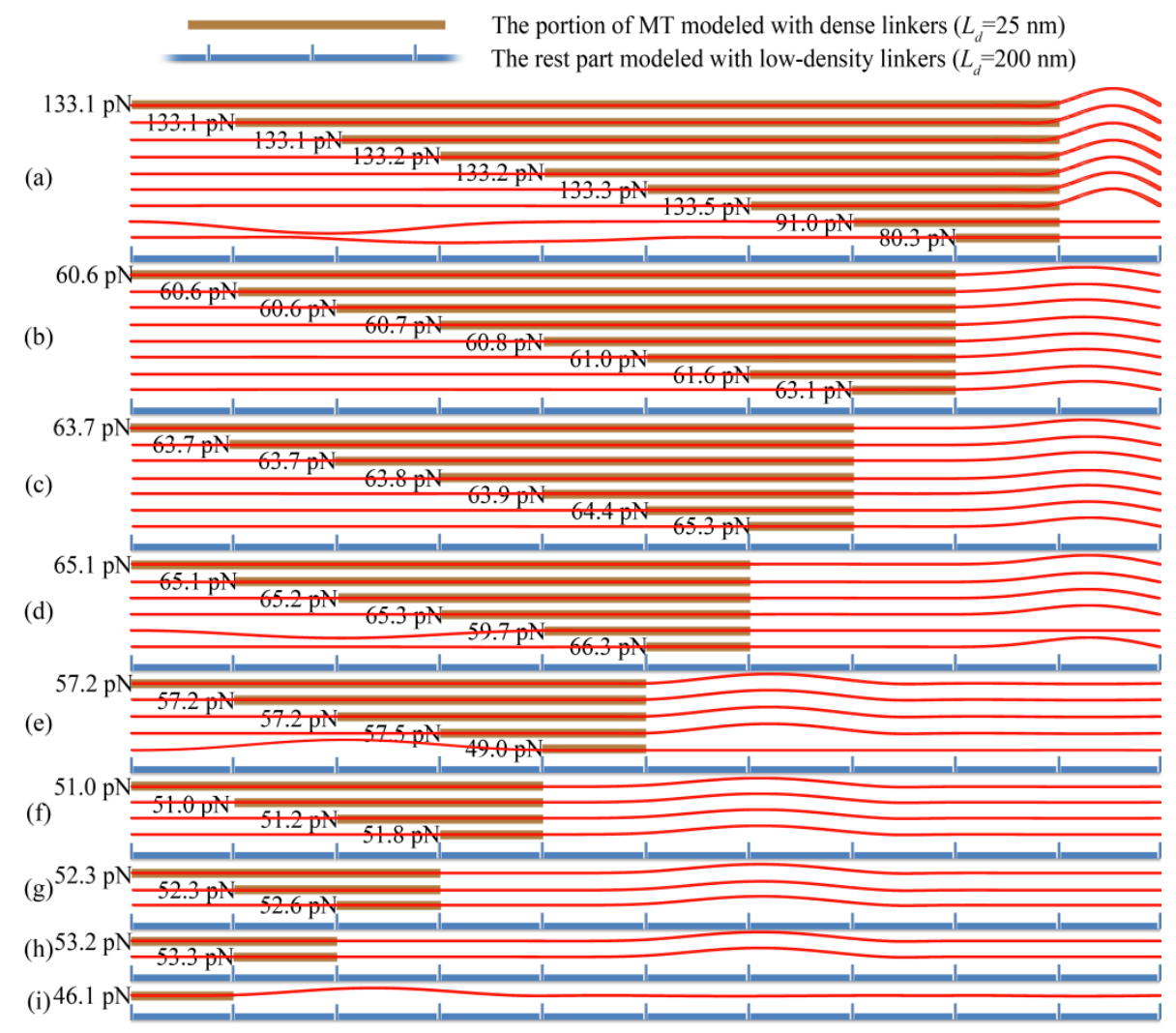

Figure 5.7 The effect of the local dense linkers on the buckling modes and associated critical loads. 


\subsection{Effect of Binding Proteins on Filopodial F-Actin}

In this section, the effect of ABPs on filopodial F-actin was investigated. The polymerizing actin bundle generates force for protrusion of the leading edge in motile cells (Fig. 5.8(a)). As shown in Fig. 5.8(a), though the length of filopodia is only about 1 to $5 \mu \mathrm{m}$ [270], the entire length of the protrusive actin bundle inside filopodial could be of the order of $10 \mu m$ [258, 271, 272]. Therefore, to study the buckling or stall force of protrusion, we considered F-actin with the length varying from 0.5 to $30 \mu m$.

To explore the buckling behaviour and obtain the critical buckling load for the filopodial F-actins, we considered two types of the filaments found in experiments whose angular separations are $166.15^{\circ}$ and $167.14^{\circ}$, respectively. Herein, the buckling deformation and the length-dependence of the critical buckling load $N_{c r}$ were shown for the F-actins with and without ABPs in Fig. 5.8(b). As plotted in Fig. 5.8(b) for a single F-actin without $\mathrm{ABP}$ support, $N_{c r}$ generally decreases with rising length. Specifically, the curves associated with two angular separations nearly coincide with each other. In other words, the angular separation does not exert significant influence on the buckling of the filaments.
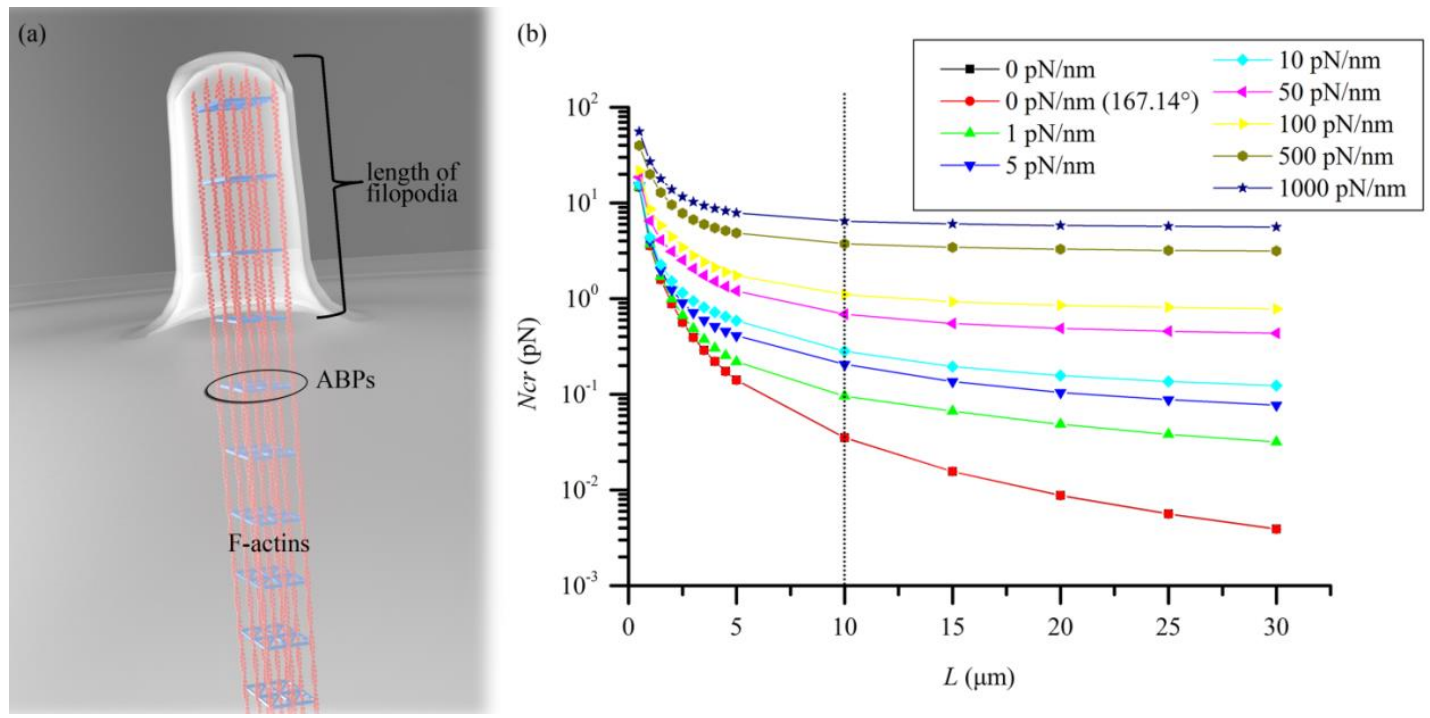

Figure 5.8 (a) The actin bundle which generates force for protrusion of the leading edge in motile cells and (b) Length-dependence of the critical buckling forces $N_{c r}$ obtained for the F-actins supported by ABPs with different extensional stiffness. 
It was reported in Ref. [258] that the bundles of parallel F-actin such as those found in filopodia cannot automatically cooperate in a linear manner to increase the amount of force generated at the bundle tip. Only the longest one in the bundle is in contact with the barrier, which thus solely resist the axial load [258]. It can be estimated from Ref. [258] that the length of the load-bearing F-actin was about 10 $\mu m$, which, in Fig. 5.8(b), corresponds to $N_{c r}$ about $0.035 \mathrm{pN}$ of a single F-actins. This however is nearly two orders of magnitude lower than the experimentally measured stall force of the order of $1 \mathrm{pN}$ [258]. Thus, the single F-actin model (See Sec. 2.3) is unable to explain the large force measured on the tip of the F-actin. The possible stiffening effect of the ABPs has to be taken into consideration by coupling them with the F-actin model (See Sec. 5.2.3). In Fig. 5.8(b), a similar trend of $N_{c r}$ was observed for the F-actins in the presence or absence of the ABPs. But the ABPs were found to be able to largely enhance the critical buckling load. This effect of the ABPs turns out to be more substantial for slender F-actins whose length is greater than $1 \mu \mathrm{m}$. For example, considering the F-actin with length $10 \mu \mathrm{m}$ the ABPs of 500 $\mathrm{pN} / \mathrm{nm}$ can raise $N_{c r}$ from $0.035 \mathrm{pN}$ to $3.750 \mathrm{pN}$ by more than an order of magnitude. Another determinant of the stiffening effect is the equivalent extensional stiffness of the ABPs. As expected, Fig. 5.8(b) shows that $N_{c r}$ of the F-actin grows considerably with the increasing stiffness. The stiffening effect of ABPs is strong when their equivalent stiffness rises up to $50 \mathrm{pN} / \mathrm{nm}$. In this process, $N_{c r}$ rises by 1 to 2 orders of magnitude when the length greater than $10 \mu \mathrm{m}$ was considered. In particular, for relatively long F-actin with length larger than $10 \mu \mathrm{m}, N_{c r}$ is of the order of $1 \mathrm{pN}$ when the modulus falls in the range of $[50 \mathrm{pN} / \mathrm{nm}, 1000 \mathrm{pN} / \mathrm{nm}]$. It is noted that organization of F-actins into different functional networks is regulated by a variety of ABPs [273], whose extensional stiffnesses range from $1 \mathrm{pN} / \mathrm{nm}$ to $1000 \mathrm{pN} / \mathrm{nm}$ [262]. However, more efforts in experimental studies still need to be made to obtain the exact value of the stiffnesses of the fascins, which are the bundlers in filopodia [273]. Thus, it is evident that the stiffnesses achieved in the present study are reasonable as 
they fall into the range of the stiffnesses of ABPs in the literature [262], although solid data on the stiffnesses of filopodial ABPs is still required to perform further comparison. Also, the MSM model is in agreement with the experiment in measuring the critical buckling load or a stall force of F-actin bundles [258].

To summarise, the above analyses show that the influence of ABPs results in a complex buckling deformation pattern, referred to as 'localized buckling' in Ref. [249], which is associated with a high critical buckling load relative to the buckling load of single F-actin without the ABPs (Fig. 5.8(b)). The ABPs can firmly hold the neighbouring F-actins and largely prevent the filaments from sliding towards each other. The stabilizing effect of ABPs is very strong, which greatly enhances the structural stiffness of F-actins and accordingly, enable them to elongate to an extent required for the filopodial protrusion before reaching the stall force. In other words, the ABPs play an essential role in regulating the stiffness of F-actin bundles. The present MSM simulations thus identify the indispensable role of the ABPs in the filopodial protrusion and cell motility [271], provide a theoretical interpretation for the experimentally achieved stall force on the F-actin and propose the physical mechanism of the filopodial protrusion [258]. Here, the present study is focused on the mechanics of individual F-actin or its bundle. However, it is noted that cytosol may exert significant effect on the mechanical behaviours of F-actin. This issue thus deserves to be examined in detail in future study.

\subsection{Summary}

The effect of the cross-linkers (subcellular environment effect) was examined for the buckling of MTs in cells. The transition from planar buckling to 3D buckling was captured and the associated increase was observed in the critical buckling load due to the increase of the 3D-linkers. The 3D buckling modes were found to be in accordance with existing experimental observations. For the 3D-linkers, the distribution of the radial orientation in the circumferential direction of MTs is 
identified as a determinant for MT buckling in cells. The more uniform radial distribution generally leads to a higher critical buckling load. Such an effect turns out to be more significant for the MTs supported by the 3D-linkers with higher density. Another determinant of MT buckling is found to be the distribution of the linker density along the axial direction of MTs. Inhomogeneous distribution give rise to the localized MT buckling limited to the region of the low-density linkers. In particular, the critical load is very sensitive to the size variation of the low-density region close to the loaded end of MTs. The size of the dense-linker region and the average linker density, however, do not have a strong influence on MT buckling.

On the other hand, the effect of binding proteins on filopodial F-actin was examined. For an F-actin bundle in the filopodial protrusion, the compressive load from cell membrane can be taken by only one F-actin supported by the ABPs that enhance the critical buckling load by one to two orders of magnitudes. The achieved buckling load is of the order of $1 \mathrm{pN}$ consistent with the experimentally measured stall force on the tip of the F-actin. The ABPs thus plays a crucial role in regulating the stiffness of F-actin bundles, facilitating the formation of the filopodial protrusion and thus, cell motility. 


\section{Chapter 6 Electromechanical vibration of CSK filaments}

\subsection{Introduction}

MTs are composed of heterodimer subunits of $\alpha$ and $\beta$ tubulins which carry unbalanced negative charge [274, 275]. With this biophysical property of tubulin MTs are able to respond to the external electric field (EF) [276, 277]. It is shown in the literature that the vibration of MTs has attracted considerable attention in the last two decades [37, 57, 60, 80, 278-280]. Recently, the electromechanical vibration of MTs was excited by applying an external EF [281], showing the potential of polarized MTs as biosensors in disease diagnosis and health monitoring. It thus becomes essential to gain an in-depth understanding of the electromechanical vibration of MTs subject to an EF [241, 281]. A theoretical study was first conducted to quantitatively describe the EF-generated by a vibrating MT [277], which provides a means to detect the vibration of MTs. After that, an optomechanical technique [281] was proposed to probe and more importantly, stimulate the vibration via EF on MTs modelled as one-dimensional structure. However, MTs are 3D discrete structures composed of charged subunits. It is thus of great interest to explore the behaviour of EF-induced vibration of MTs as 3D nanostructures. In existing studies, vibration analyses were carried out for MTs first in the framework of continuum mechanics theory [37]. Subsequently, discrete approaches were developed to account for nanoscale MT structure and its influence on MT vibration [80]. The typical examples are the MD simulations [69, 72, 175] and the cost-effective MSM model [77].

In this chapter, firstly (Sec. 6.2), the MSM model [77] was employed to simulate the vibration of individual MTs subject to an alternating EF generated by a dipole antenna (Fig. 6.1). The vibration spectra were recorded for MTs for various possible modes. In addition, the correlation between frequency shift and the possible softening/hardening of MTs was quantified demonstrating the potential application of 
vibrating MTs as biosensors. In addition, a preliminary study was also conducted to evaluate the damping effect of surrounding cytosol, which could possibly be reduced by the nanoscale MT-cytosol interface. Subsequently in Sec. 6.3, the effect of nanoscale MT-cytosol interface on cytosol damping was further investigated in a coupled study of MD and MSM simulations. The MSM simulations were carried out with a desktop computer with 4 cores and the MD simulations were carried out in a cluster computer with 56 cores.

As mentioned earlier, the MT vibration in response to EF could open a door to the MT-based biosensors. In spite of this, it is argued that cytosol damping in cells would quench the vibration when a non-slip solid-liquid interface is assumed for the MT-cytosol system [282, 283]. In other words, MT vibrations can survive in cells only when the cytosol damping is largely reduced by the nanoscale MT-cytosol interface due to, e.g., a slipping ionic layer near MT surface [58, 241, 278]. In addition, the solid-liquid interface at the nanoscale is found to be different from its macroscopic counterpart as the vdW interaction at the interface is significant due to the largely increased surface-to-volume ratio. It was reported in experiments that a CNT submerged in water can still vibrate in radial breathing modes (RBMs) with frequency raised by the vdW interaction between CNT and water [284, 285]. For similar reasons, there may exist a gap between MTs and cytosol due to the repulsive $\mathrm{vdW}$ force, which would largely decrease the cytosol damping on MT vibration. Indeed, the megahertz mechanical vibration of MTs was already detected in the wet and noisy subcellular environment of neuronal cells [286]. Motivated by this idea, we aim to study the MT-cytosol interface by considering the vdW force and the static electrical force at the interface and examine the influence of such an interface on the cytosol damping of MT vibration. Indeed, the unique features of the nanoscale solid-liquid interface and its effect on the dynamic behaviour of MTs are a major topic of great interest in current research of nano- and bio-mechanics.

Thus in Sec. 6.3, the effect of the interface on the electrically excited vibration 
of MTs in cytosol (80\% water) was investigated. MD simulations were employed to study the interface between MT and water. The obtained properties were then entered into an MSM model of MTs with updated values of force constants [56, 77] to examine the effect of MT-cytosol interface on the collective vibration of MTs. The MT vibration in this study was also excited by an alternating EF from a dipole antenna (Fig. 6.1).

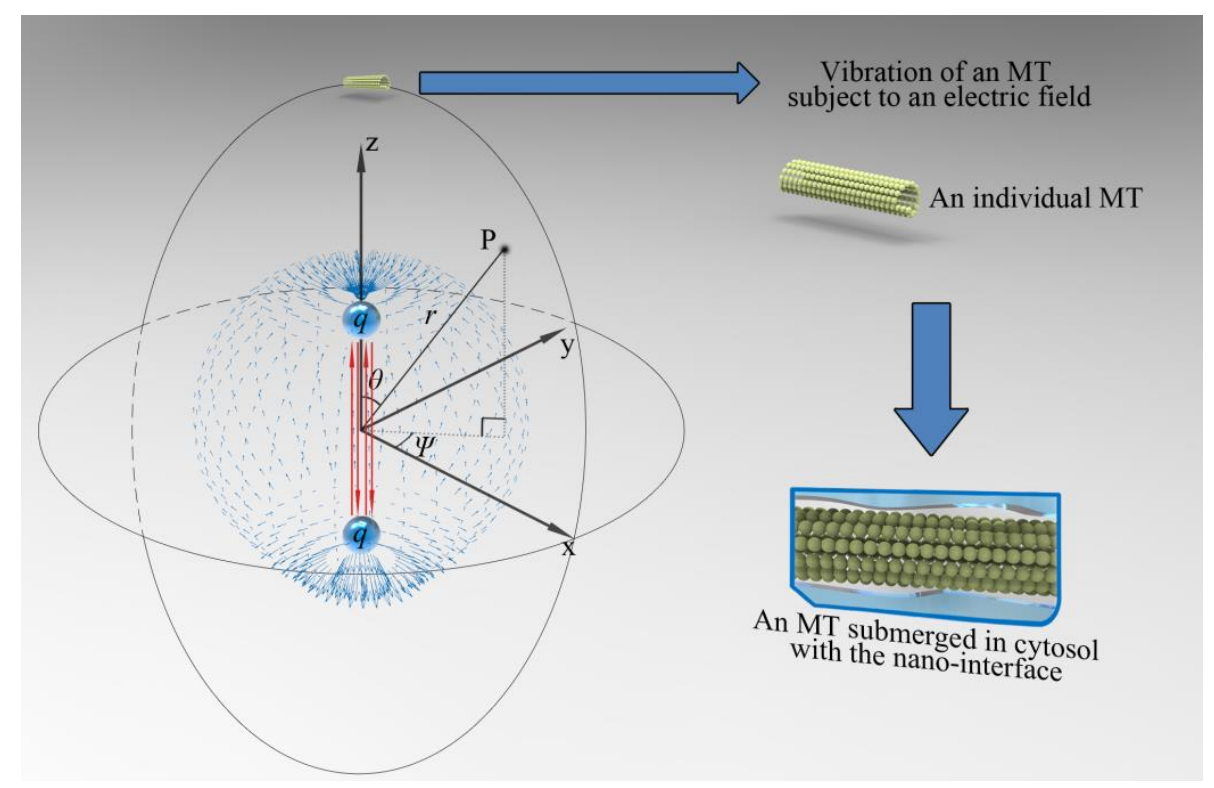

Figure 6.1 Illustration of an individual MT and an MT-cytosol system subject to an electric field generated by a dipole antenna

\subsection{Electric excited vibration of $\mathrm{MT}$ and its application in biosensors}

\subsubsection{Modelling of excitation and damping of MT vibration}

\subsubsection{Modelling of EF excited vibration of MT}

The migration of individual MTs in an EF has been observed via video contrast microscopy [274]. In this process, the magnitude of the unbalanced negative charge carried by tubulins was found to be lower than the theoretical one due to the effects of the electroosmotic flow and the surrounding substances [274]. Also, this magnitude varies from one experiment to another [274, 287-289]. On the other hand, 
the computer simulations from crystallographic data give an identical value of -5 electron charges for the tubulin monomer isolated from environment [274, 290, 291]. As such, the charge carried by one monomer was set to $q_{t}=-5 \mathrm{e}$ [291] in the present study. Fig. 6.1 shows an alternating EF generated by a Hertzian Dipole in a spherical polar coordinate system characterised by the radial $(r)$, polar angle $(\theta)$ and azimuthal angle $(\Psi)$ coordinators. The tubulin monomer with negative charges can respond to the electromagnetic field generated by a Hertzian Dipole in this coordinate system [292-294]:

$$
\begin{gathered}
E_{r}=j \frac{p_{0}}{4 \pi \varepsilon} 2 K_{w} \cos \theta\left(1-\frac{j}{K_{w} r}\right) \frac{e^{j\left(\omega t-K_{w} r\right)}}{r^{2}} \\
E_{\theta}=-\frac{p_{0}}{4 \pi \varepsilon} K_{w}{ }^{2} \sin \theta\left(1-\frac{j}{K_{w} r}-\frac{1}{K_{w}{ }^{2} r^{2}}\right) \frac{e^{j\left(\omega t-K_{w} r\right)}}{r}
\end{gathered}
$$

where $K_{w}=\omega / c, \omega$ is the angular frequency, $c$ is the speed of light, $\varepsilon$ is the permittivity, $p_{0}$ is the dipole moment of the Hertzian Dipole.

Thus the EF was described as $\vec{E}=E_{r} \hat{r}+E_{\theta} \hat{\theta}$ and in order to simplify the calculation, the MT was placed ( $1 \mu \mathrm{m}$ in length) at the coordinate of $\theta=0$ and $r=$ $0.1 \mathrm{~m}$ with the EF vector parallel or perpendicular to the axial direction of MT. When the radial distance $r$ of the MT is much greater than its length, the EF can be approximated as a uniform EF exhibiting harmonic time dependence. Also here defined a $\gamma_{i n t}$ as $\gamma_{i n t}=p_{0} / \varepsilon$ to control the intensity of EF by adjusting the value of $|\vec{E}|$. For instance, in the present study a $\gamma_{i n t}=0.01 \mathrm{~V} \cdot \mathrm{m}^{2}$ was considered to produce an EF with an amplitude $A_{E}$ of $20 \mathrm{~V} / \mathrm{m}$ (the corresponding unit of vibration amplitude will be $1 \mathrm{~nm})$. As the vibration amplitude of MT $A_{M T}$ varies linearly with $A_{E}$, the $A_{M T}$ presented in the present chapter could be dimensionless (i.e., the unit of amplitude will be $1 \mathrm{~nm}$ when $A_{E}$ is $20 \mathrm{~V} / \mathrm{m}$, the unit will be $1 \mu \mathrm{m}$ when $A_{E}$ equals to $20 \times 10^{3} \mathrm{~V} / \mathrm{m}$ ). Then a fixed-fixed condition was considered to be applied to MT with a fixed length of $L_{f i x}=5 a_{m}$ on both ends ( $a_{m}=4.05 \mathrm{~nm}$ is unit repeat of monomer). And the above mentioned EF applies the sinusoidal force on the tubulins of MT via the equation $\boldsymbol{F}=\vec{E} q_{t}$. Here, Fig. 6.1 graphically illustrates that an alternating EF is generated by 
an electronic device (represented by a dipole here) and used to excite the vibration of individual MTs which are a distance away from the electronic device. The frequency shifts of MTs can then be employed as a biomarker to monitor the changes of MT structure and stiffness in pathological and physiological processes. Thus, the distance $r=0.1 \mathrm{~m}$ selected here between the dipole and MTs could demonstrate such a design of the MT-based biosensor. In the meantime, it is understood that the amplitude of time-dependent EF should be large enough to excite the forced vibration of MTs with sufficiently large amplitude and thus, easy to identify by e.g., vibration energy absorption. The safety is another issue that needs to be taken into consideration. Therefore, $|\vec{E}|=20 \mathrm{~V} / \mathrm{m}$ was selected as it is relatively strong but safe for human being according to World Health Organization and Federal Office for Radiation Safety, Germany [295]. In this study, the vibration analysis was carried out based on the MSM model [77] developed for 13-3MTs as shown in Chapter 2. The details for dynamic vibration analysis with MSM model was provided in Sec. 6.2.1.3.

\subsubsection{Brief introduction to the preliminary evaluation of damping}

Moreover, a preliminary parametric study was conducted for the transverse vibration of MTs in cytosol across a range of damping effects and the frequencies up to $50 \mathrm{MHz}$. The damping force $F_{d}$ on the MT monomers was applied to characterise the energy dissipation in viscous flow of cytosol and calculated based on the slide film damping theory accounting for the damping effect of microfluid between two moving microscale objects [296, 297] (See details in Sec. 6.2.1.3). First, a non-slip boundary condition associated with a non-slip MT-cytosol interface was considered in calculating $F_{d}$. After that, damping reduction coefficient $P$ from 0.0001 to 0.1 was introduced to estimate the reduced damping effect. i.e., the damping force on the monomers decreases to $F_{R d}=F_{d} \times P$. Meanwhile, the quality factor $Q=\omega_{m} / \triangle \omega$ was also calculated for the damped MT vibration, where $\omega_{m}$ is the resonant frequency, $A_{m}$ is the amplitude of the resonance peak for damped vibration and $\Delta \omega$ is the 
frequency increment at the points with amplitude $A_{m} / \sqrt{2}$. [298, 299]. Here it should be pointed out that these calculations could provide insights into how much reduction is required to achieve prominent MT vibration in cells. The possible reduction of cytosol damping may originate from the relative sliding [241] and/or the vdW interaction between MT and cytosol, i.e., a nanoscale MT-cytosol interface. Other factors may also exert influence on the damping of cytosol. For example, in [300] the wettability and especially, the slip length was identified as major factor affecting water damping effect on the longitudinal vibration of polarized nanorods. However, the reduction of cytosol damping due to these factors has not been examined systematically for all the possible vibration modes based on experiments or atomistic simulations.

\subsubsection{Supplementary details of the model}

The details of the mathematical techniques used in vibration analyses were introduced in the following contents. Specifically, the dynamic analysis for MSM model (Structure under loads that vary sinusoidally at the known frequency) was introduced as follows.

The high-frequency dynamics analysis follows the general equation of motion for a structural system. Consider the general equation of motion for a structural system

$$
\mathbf{M} \ddot{\boldsymbol{u}}+\mathbf{C} \dot{\boldsymbol{u}}+\mathbf{K u}=\boldsymbol{F}
$$

where $\ddot{\boldsymbol{u}}$ denotes the acceleration vector, $\dot{\boldsymbol{u}}$ denotes the velocity vector, $\boldsymbol{u}$ denotes the nodal displacement vector, $\boldsymbol{F}$ denotes the applied force vector, $\mathbf{M}$ denotes the structural mass matrix, $\mathbf{C}$ denotes the structural damping matrix, $\mathbf{K}$ denotes the structural stiffness matrix.

As stated above, all points in the structure are moving at the same known frequency, however, not necessarily in phase. Also, it is known that the presence of damping causes phase shifts. Therefore, the displacements could be defined as 


$$
\boldsymbol{u}=\left\{u_{\max } e^{j \varphi}\right\} e^{j \omega t}
$$

where $u_{\max }$ denotes the maximum displacement, $j$ denotes square root of $-1, \omega$ denotes imposed circular frequency (radians/time) $=2 \pi f, f$ denotes the imposed frequency (cycles/time), $t$ denotes time, $\varphi$ denotes the displacement phase shift (radians).

The above equation can be written as $\boldsymbol{u}=\left\{u_{\max }(\cos \varphi+j \sin \varphi)\right\} e^{j \omega t}$ or as $\boldsymbol{u}=\left(\left\{u_{1}\right\}+j\left\{u_{2}\right\}\right) e^{j \omega t}$.

where $\left\{u_{1}\right\}=\left\{u_{\max } \cos \varphi\right\}$ is the real displacement vector and $\left\{u_{2}\right\}=\left\{u_{\max } \sin \varphi\right\}$ is the imaginary displacement vector.

The force vector can be specified analogously to the displacement:

$$
\begin{gathered}
\boldsymbol{F}=\left\{F_{\max } e^{j \psi}\right\} e^{j \omega t} \\
\boldsymbol{F}=\left\{F_{\max }(\cos \psi+j \sin \psi)\right\} e^{j \omega t} \\
\boldsymbol{F}=\left(\left\{F_{1}\right\}+j\left\{F_{2}\right\}\right) e^{j \omega t}
\end{gathered}
$$

where $F_{\text {max }}$ denotes force amplitude, $\psi$ denotes force phase shift (radians), $\left\{F_{1}\right\}=\left\{F_{\text {max }} \cos \psi\right\}$ is the real force vector, $\left\{F_{2}\right\}=\left\{F_{\max } \sin \psi\right\}$ is the imaginary force vector .

Then we could have

$$
\left(-\omega^{2} \mathbf{M}+j \omega \mathbf{C}+\mathbf{K}\right)\left(\left\{u_{1}\right\}+j\left\{u_{2}\right\}\right) e^{j \omega t}=\left(\left\{F_{1}\right\}+j\left\{F_{2}\right\}\right) e^{j \omega t}
$$

The dependence on time $\left(e^{j \omega t}\right)$ is the same on both sides of the equation and may therefore be removed and we have

$$
\left(\mathbf{K}-\omega^{2} \mathbf{M}+j \omega \mathbf{C}\right)\left(\left\{u_{1}\right\}+j\left\{u_{2}\right\}\right)=\left(\left\{F_{1}\right\}+j\left\{F_{2}\right\}\right)
$$

Solving this equation can help us obtain the amplitude-frequency spectrum of MT excited by periodic sinusoidal electric force.

For the transient dynamics solution of electric excited vibration of MTs, the FE semi-discrete equation of motion (Eq. 6.2) can be denoted as

$$
\mathbf{M} \ddot{\boldsymbol{u}}(t)+\mathbf{C} \dot{\boldsymbol{u}}(t)+\mathbf{K u}(t)=\boldsymbol{F}(t)
$$

And the displacement-time relationship can be obtained following the classic numerical time integration method [301]. 
In this study, we considered the damping effect of cytosol from damping force applied on MTs. Therefore, the structural damping matrix $\mathbf{C}$ was ignored. The method to obtain the structural mass matrix $\mathbf{M}$ and the structural stiffness matrix $\mathbf{K}$ was introduced in Sec. 2.2.3.

It is worth mentioning that in this study, the values of $k_{i}^{r}, k_{i}^{\varphi}, k_{i}^{\tau}$ were obtained from the MD simulations in [70, 77, 167] and further optimized in [56], i.e., $k_{1}^{r}=3 \mathrm{nN} / \mathrm{nm}, \quad k_{1}^{\varphi}=2 \mathrm{nN} \cdot \mathrm{nm}, \quad k_{1}^{\tau}=0.04 \mathrm{nN} \cdot \mathrm{nm}, \quad k_{2}^{r}=0.14 \mathrm{nN} / \mathrm{nm}, \quad k_{2}^{\varphi}=0.085 \mathrm{nN} \cdot \mathrm{nm}$, $k_{2}^{\tau}=0.0017 \mathrm{nN} \cdot \mathrm{nm}$.

In the vibration analyses, the damping effect of cytosol on MT vibration was characterised by the model based on the slide film damping theory.

The model considers the energy dissipation due to viscous flow of cytosol. Specifically, the slide film damping theory was utilized here to characterise the energy dissipation due to the viscous flow in cytosol during the high-frequency vibration of MTs. The damping effect caused by microfluid between two moving microscale objects can be effectively represented by this theory [296, 297]. As can be seen from the experimental observation of a cell, the MTs in cytosol are surrounded by microscale objects [255]. During the high-frequency vibration, the relative motion between the MT and the surfaces will also lead to the energy dissipation in cytosol and the damping of the vibration. The governing equation obtained for the sinusoidal motion $\left(v=v_{0} \cos (\omega t)\right)$ of the subunit of MT relative to the surrounding surfaces is as follows [297]:

$$
\rho \frac{\partial v}{\partial t}=\eta \frac{\partial^{2} v}{\partial z_{h}^{2}}
$$

where $v$ is the velocity, $\rho$ is the density of fluid and $\eta$ is the dynamic viscosity of the fluid $\left(6.97 \times 10^{-4}\right.$ Pa.s [241]), $z_{h}$ is the distance between a point in fluid and the moving solid surface.

In this theory, the transition from Couette to Stokes flow occurs at a cut-off frequency [296] $f_{c}=\eta / 2 \pi \rho h_{s}{ }^{2}$, where the $h_{s}$ is the spacing between the MT and adjacent filaments and set to $20 \mathrm{~nm}$ here [106]). Thus, $277 \mathrm{MHz}$ was obtained as the 
cut-off frequency which is far beyond the scope (1-100 MHz) considered and shows that the Couette flow model is suitable for the cytosol around the vibrating MT.

The solution of Eq. 6.10 gives the velocity profile of the fluid field. And with the Newton's law of viscosity [302] and the momentum transport [303], damping forces will be calculated as $F_{d}=\eta A_{t w} v_{t} / h_{s}$ [296], where $v_{t}$ is the velocity of tubulin during vibration and $A_{t w}$ is the tubulin-water contact area $\left(A_{t w} \approx 23.5 \mathrm{~nm}^{2}\right.$ calculated as the product of tubulin unit repeat and the width from Ref. [77]).

In this section (Sec. 6.2), two types of MT-cytosol interfaces were assumed and compared with each other in terms of damping effect, i.e., (1) the non-slip boundary condition associated with a non-slip MT-cytosol interface where $u_{\text {liquid }}=u_{\text {solid }}$ [304] and the damping forces $F_{d}$. (2) the slip-boundary condition at the interface where $u_{\text {liquid }} \neq u_{\text {solid }}$ and the damping force is $F_{d} \times P$ on the monomers. Here, coefficient $P$ ranges from 0.0001 to 0.1 , reflecting different reduced damping effect.

\subsubsection{Characterisation of the electromechanical vibrations}

In the following contents, the forced vibrations of individual MTs subject to an alternating EF were explored, and the influence of the abnormal interaction between adjacent tubulins was examined. In addition, a preliminary study was also conducted to evaluate the influence of the reduced cytosol damping due to the distinctive MT-cytosol interface at the nanoscale.

\subsubsection{Vibrations excited by transverse electric field}

As mentioned in Sec. 6.1, the investigations on the free vibration of MTs have achieved multiple advances [37, 60, 305]. The Eigenvalue vibration analysis was efficiently used to predict the vibration modes and resonant frequencies of MTs [37, $78,80]$. The associated vibration amplitude however cannot be obtained by solving the Eigenvalue problems. In this study, we further studied the forced vibrations of MTs excited by a uniform EF. The major concerns are (1) the possible vibration modes and associated frequencies that can be excited by an alternating $\mathrm{EF}$ in the 
transverse or axial direction of MTs, and (2) "vibration amplitude-EF frequency" relations, which is crucial in predicting the equivalent dipole moment of vibrating MTs [277] and facilitating the design of MT-based biosensors based on the electromechanical vibration of MTs [241].

First, the forced vibrations of MTs excited by the transverse alternating EF (Fig. 6.1) were presented in Fig. 6.2 in comparison with the free vibrations obtained for the same MT via the Eigenvalue analysis.

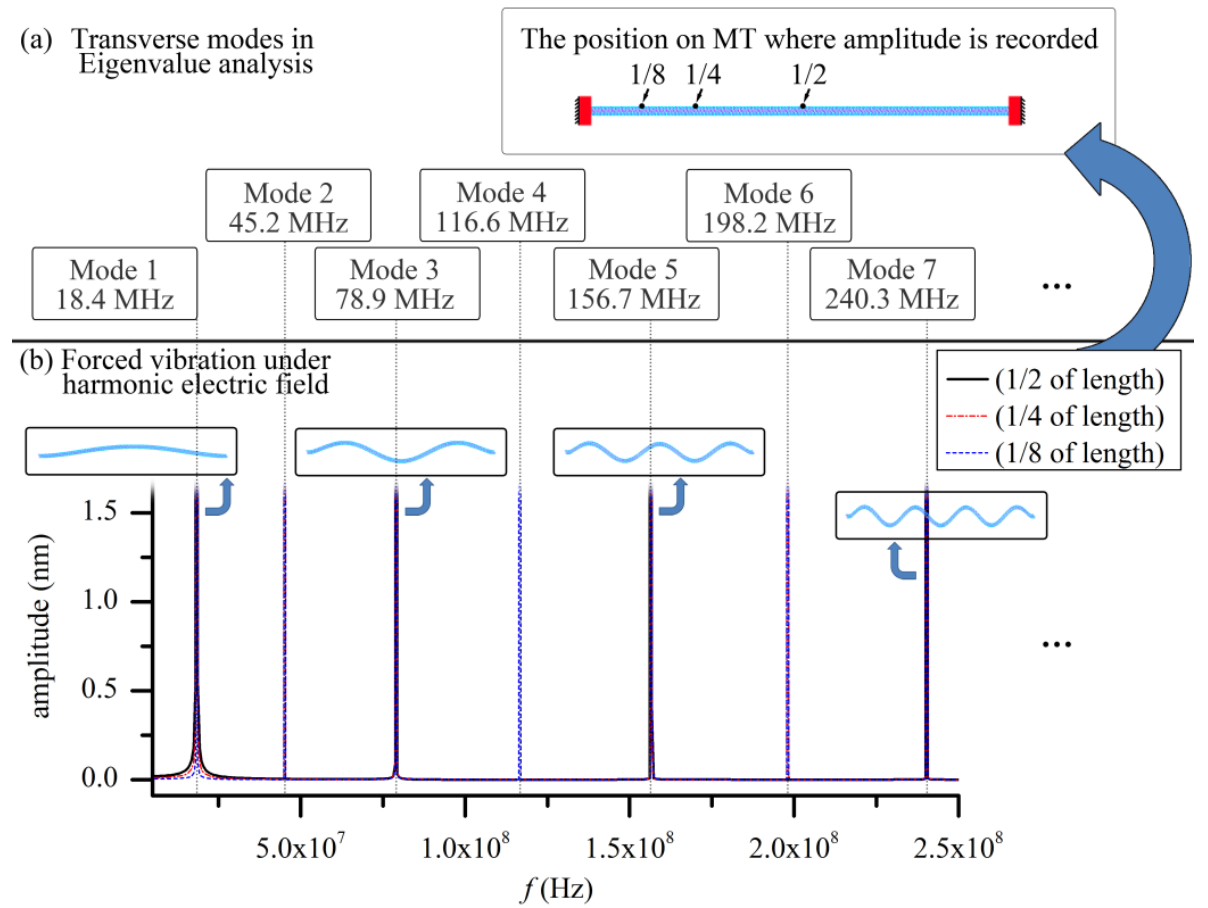

Figure 6.2 The comparison between the Eigenvalue transverse vibration modes and the vibrations of MTs excited by the EF in the transverse direction

The top panel of Fig. 6.2 shows seven transverse free vibrations whose resonant frequency up-shifts with the growing half wavenumber and falls in the range of $(0 \mathrm{MHz}, 250 \mathrm{MHz})$. The amplitudes of the MT were measured at the three points whose distance from the left MT end is $L / 2, L / 4$ and $L / 8$, respectively ( $L$ is the MT length) (Fig. 6.2). In the obtained amplitude-frequency spectra (Fig. 6.2) seven peaks were picked up in the vicinity of the resonant frequencies. Accordingly, seven forced transverse modes were achieved, which are identical to the seven free vibration modes shown in Fig. 6.2. Meanwhile, for a given amplitude the width of frequency 
range $\left(W_{f r}\right)$ of the peaks (the frequency span around the frequency peak with the amplitude greater than the given value) associated with the odd half wavenumbers 1 , 3,5 and 7 , are found to be greater than those corresponding to the even half wavenumbers 2, 4 and 6 . In addition, among the peaks with an odd half wavenumber, the $W_{f r}$ increases with decreasing wavenumber or declining frequency. The observation suggested that the excitation of the MT vibration with relatively large odd half-wavenumber and particularly, even half-wavenumbers are very sensitive to the selected frequency of the EF, and accordingly, show narrow or sharp peaks in the amplitude-frequency spectra. They can be obtained only if the frequency of excitation is the same as the corresponding resonant frequency of MTs. A small deviation from the resonant frequency would extinguish these even modes. It follows that the odd transverse modes of MTs with low frequency can be excited in a wider range of frequency and thus, are promising for their applications in the MT-based biosensors.

\subsubsection{Vibrations stimulated by axial electric field}

Possible vibration behaviours of MTs other than transverse modes have been predicted by Eigenvalue analysis [37, 277]. Such vibration modes can also be achieved by applying an alternating EF in the axial direction (Fig. 6.3(a)). Fig. 6.3(b-c) and $d$ show the three typical examples and the associated amplitude-frequency spectra. The RBM with a circular cross-section and the axial half wavenumber $m=1$ was observed at around 53.019MHz (Fig. 6.3(a)). In RBM, the $\alpha$ and $\beta$ tubulins of the MT oscillate in the radial direction as if the MT was breathing with a time-dependent radius. The vibrational radial displacement is distributed uniformly along the perimeter of MT cross-section. Along the MT length, the distribution however becomes non-uniform and characterised by the axial half wavenumber $m$, which increases with rising frequency. For instance, the frequency $159.291 \mathrm{MHz}$ of the RBM with $m=3$ is three times as much as the $53.019 \mathrm{MHz}$ 
associated with $m=1$. In addition, the circumferential modes with a non-circular cross-section [60, 306] were also found in Fig. 6.3(c) at around 585.639 MHz. The radial amplitudes (Fig. 6.3(a)) at the middle point of the MTs were recorded in the amplitude-frequency spectra for the vibration modes (Fig. 6.3(b-c)). Here, two peaks were observed around the resonance frequencies (of RBMs) 53.019MHz (Fig. 6.3(b)) and 585.639 MHz (Fig. 6.3(c)), respectively. The amplitude is of the order of $0.02 \mathrm{~nm}$ at $53.019 \pm 0.001 \mathrm{MHz}$ and $0.0003 \mathrm{~nm}$ at $585.639 \pm 0.001 \mathrm{MHz}$, which is two orders of magnitude smaller than the first one. Meanwhile, in Fig. 6.3(c) another peak of circumferential mode was also observed at a frequency slightly lower than 585.639 MHz of RBM.

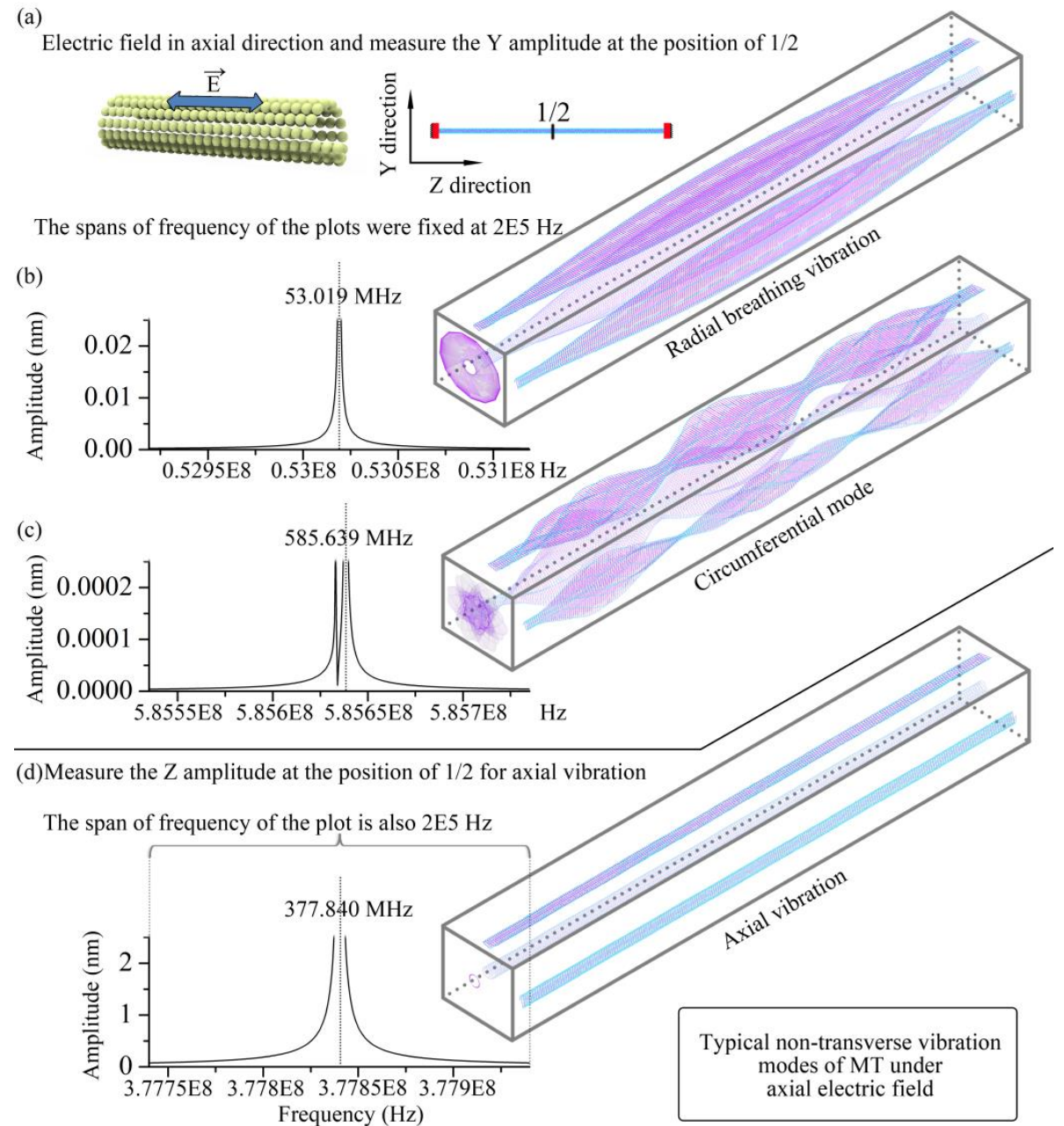

Figure 6.3 The vibration modes of MTs excited by the EF in the axial direction

In addition to RBMs, the axial vibration mode at around $377.840 \mathrm{MHz}$ possesses the highest amplitude as shown in Fig. 6.3(d). Specifically, a sinusoidal 
alternating $\mathrm{EF}$ with an amplitude of $20 \mathrm{~V} / \mathrm{m}$ can excite the axial vibration of individual MTs with a $W_{f r}$ (associated with amplitude greater than $0.1 \mathrm{~nm}$ ) around 150 $\mathrm{KHz}$, i.e., from $377.915 \mathrm{MHz}$ to $377.765 \mathrm{MHz}$. In this study, the amplitude $0.1 \mathrm{~nm}$ is selected as a reference oscillatory displacement. The $W_{f r}$ of a resonant peak is then defined as the frequency range (around the peak) associated with the amplitude greater than $0.1 \mathrm{~nm}$. This definition allows us to compare the $W_{f r}$ between different resonant peaks.

\subsubsection{Dependence of vibration on tubulin interaction}

It was shown recently [307] that cell stiffness can be used as a biomarker to identify cancerous cells among surrounding healthy cells. Herein, MTs are known as one of the major structural elements responsible for cell stiffness and the variation of MT stiffness can be reflected by the frequency shift of MT vibration excited by an EF [241]. It is thus expected that MT frequency can be used as a bioindicator to quantify the changes in the structural stiffness of MTs in diseased cells.

It is understood that the structural domains of tubulins, i.e., the N-terminal, intermediate, and C-terminal domains [308], may contribute to heterodimer stability, longitudinal and lateral PF interactions, nucleotide exchange and hydrolysis, and MT-protein interactions [308]. Disease-causing amino acid substitutions were found among the three domains of tubulins and therefore predicted to perturb a range of MT functions [309]. Specifically, the disease-causing substitutions in TUBB2B alter amino acids in domains important for GTP binding, heterodimer stability and longitudinal interactions, and those in TUBB3 are located primarily in regions that regulate GTP binding, heterodimer stability, and longitudinal and lateral interactions. It follows that the variation of MT stiffness may arise from the abnormal interactions between adjacent tubulins due to pathological changes (e.g., the above-mentioned disease-causing substitutions or the cancer progression induced structural remodelling of MTs) in human body [56]. It is thus of great interest to study the 
dependence of the elastic moduli and vibration frequency of MTs on the strength of tubulin interaction. The outcomes have potential applications in developing biosensor for the biophysical properties of individual MTs or cells.

To mimic this scenario, we have adjusted the strength of longitudinal and helical tubulin interactions by varying the values of the force constants $k_{i}^{r}, k_{i}^{\varphi}$, and $k_{i}^{\tau}$ (Sec. 2.2) that control changes in the bond length, the bond angle and the dihedral angle of tubulin interactions (Fig. 2.1(c-f)). $k_{i}^{r}, k_{i}^{\varphi}$, and $k_{i}^{\tau}$ for normal cells were obtained in $[56,70]$ and replaced in this study by $\Omega k_{i}^{r}, \Omega k_{i}^{\varphi}$, and $\Omega k_{i}^{\tau}$ where $\Omega$ is a coefficient ranging from 0.2 to 2 , which allows us to produce the elastic modulus close to the experimentally obtained stiffness of cancer cells at different progression stages (determined by atomic force microscopy) [307]. First, Young's modulus $Y$ and Shear modulus $G$ were measured for the MTs where the strength of the tubulin interactions varies by an order of magnitude. The method used here to measure the elastic moduli is demonstrated in [77]. Subsequently, the fundamental vibration was studied for the above MTs subject to a transverse EF. The aim of this study is to establish the correlation between the tubulin interaction and the $W_{f r}$ at the resonant frequency, which is associated with the frequency span with an amplitude greater than $0.1 \mathrm{~nm}$ [277].

As shown in Fig. 6.4(a), when the longitudinal interaction is altered with $\Omega$ rising from 0.2 to $2, G$ changes slightly around $1.5 \mathrm{MPa}$ [21], while $Y$ increases linearly from $0.17 \mathrm{GPa}$ to $1.71 \mathrm{GPa}$ by a factor of 10.06 . In the same process, the fundamental frequency rises from $9.02 \mathrm{MHz}$ to $23.67 \mathrm{MHz}$ (by a factor of 2.62) while the $W_{f r}$ decreases from $7.05 \mathrm{MHz}$ to $2.48 \mathrm{MHz}$ (by $65 \%$ ). The trend of the properties and dynamic responses to change with helical interaction is plotted in Fig. 6.4(b). In contrast to the longitudinal interaction effect, $Y$ remains nearly unchanged while $G$ grows linearly from $0.32 \mathrm{MPa}$ to $2.86 \mathrm{MPa}$ by a factor of 8.94 with rising $\Omega$. It also is noted in Fig. 6.4(b) that when $\Omega$ rises from 0.2 to 2, the fundamental frequency rises from $13.67 \mathrm{MHz}$ to $19.41 \mathrm{MHz}$ (by $42 \%$ ) while the $W_{f r}$ decreases from $4.30 \mathrm{MHz}$ to 
$3.03 \mathrm{MHz}$ (by $30 \%$ ). The effect of the helical interaction on the frequency and the $W_{f r}$ is found to be smaller than the effect of the longitudinal interaction.

Herein the longitudinal interaction is found to be important for axial Young's modulus, while the helical interaction is mainly responsible for Shear modulus. These results are in agreement with $[20,56]$. Consistent with this observation, the longitudinal interaction also plays a major role in fundamental (transverse) vibration, where axial Young's modulus has a predominant effect over shear modulus. Moreover, the fundamental frequency also changes with the variation of the helical interaction and is found to be more sensitive to the helical interaction weakening than its strengthening. For instance, when $\Omega$ falls from 1 to 0.2 , i.e., the helical interaction is weakened by $80 \%$, the fundamental frequency decreases by $26 \%$ while it only increases by $5 \%$ when $\Omega$ rises from 1 to 2 , i.e., the helical interaction is strengthened by $100 \%$.
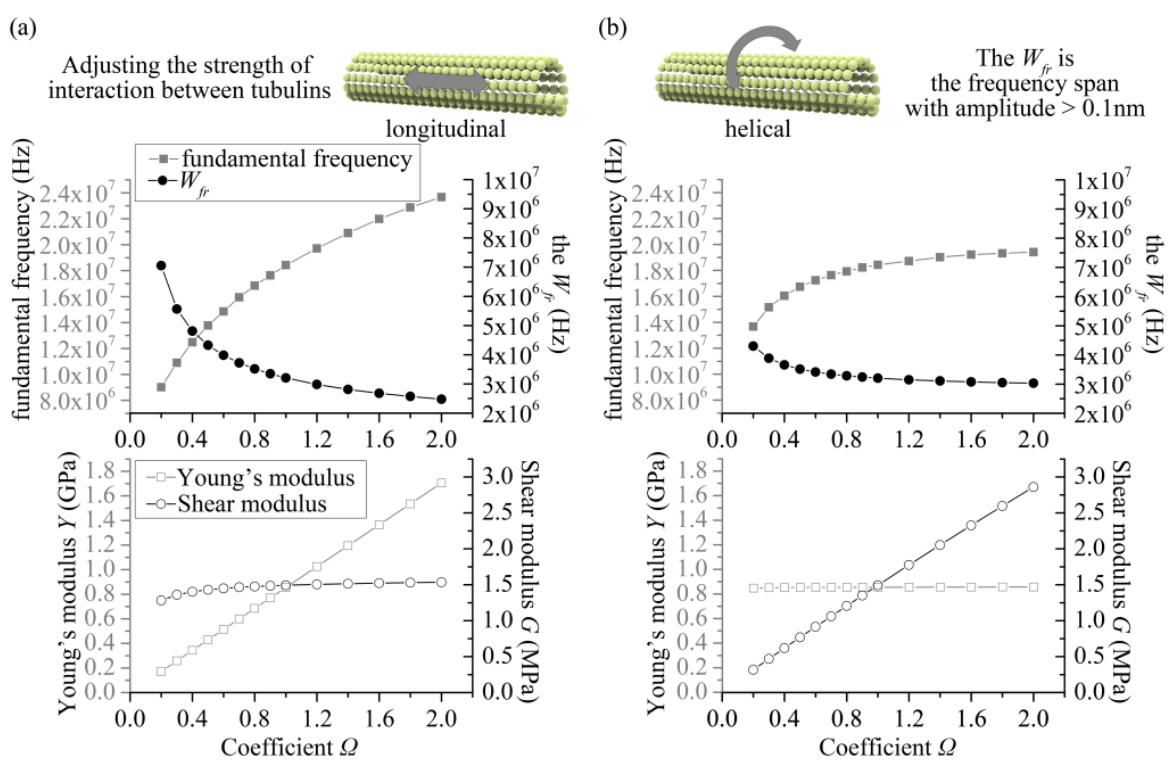

Figure 6.4 The changes in vibrational responses and elastic moduli of MTs as a result of (a) abnormal longitudinal interactions between tubulins and (b) abnormal helical interactions between tubulins

The above study indeed shows a typical example that the nanostructures of subcellular components like MTs may have a substantial influence on their overall static and dynamic responses [50]. The prominent role of longitudinal interaction in 
the transverse MT vibration originates from its influence on axial Young's modulus that controls the bending or transverse deformation of MTs. In the meantime, the helical interaction is responsible for the resistance of MTs to shear deformation or inter-PF sliding. Weakening the helical interaction leads to significant inter-PF sliding $[49,53,56]$, which however is prohibited when the interaction is strengthened. This explains the relatively large frequency shift due to the helical interaction weakening. These results obtained in the present simulations show the potential of polarized MTs as a biosensor and its frequency as a possible biomarker in detecting the property and structural changes in individual MTs or cells in physiological or pathological processes.

\subsubsection{Preliminary evaluation of reduced cytosol damping}

In previous sections, the EF-excited vibrations of individual MTs were investigated without considering any damping effect in the intracellular environment. In reality, MTs are submerged in cytosol of cells (80\% water). Thus, cytosol damping cannot be avoided. On the other hand, the damping effect may reduce to some extent due to, e.g., the possible MT-cytosol sliding and the vdW interaction although we are still waiting for experiments or simulations to confirm this assumption. Therefore, it is of interest to conduct a parametric study of cytosol damping and find the condition under which the prominent vibration of MTs can be excited by EF. It was shown in Sec. 6.2.1.2 that a coefficient $P$ was introduced to decrease the frictional forces on the MT due to surrounding cytosol and thus, measure the reduction of the damping effect. The amplitude-frequency spectra are calculated in Fig. 6.5 for the transverse vibrations with $P$ rising from 0.0001 to 1 . For the non-slip MT-cytosol interface with $P=1$, viscous damping is so strong that can extinguish the vibrations of MTs, which is found to be in agreement with [282]. When the damping effect decreases to $P=0.1$, the amplitude increases but is still very small. Further decreasing $P$ to $0.01,0.001$ and to 0.0001 leads to the increasing amplitude of the order of $0.02 \mathrm{~nm}, 0.2 \mathrm{~nm}$ and 
$1.2 \mathrm{~nm}$, respectively. Thus, transverse vibration with amplitude greater than $0.1 \mathrm{~nm}$ [277] can be achieved when the cytosol damping effect can decrease by three orders of magnitude due to the unique features of the nanoscale MT-cytosol interface. To reveal the trend of quality factor $Q$ in this process we have calculated the frequency spectra with $P$ ranging from 0.002 to 0.009 in the inset of Fig. 6.5. In the figure, $Q$ is found to increase with decreasing damping effect measured by $P$ and at $P<0.003 Q>$ 4.3 can be obtained. Here, it is worth mentioning that decreasing cytosol damping leads to greater $Q$ mainly by raising the amplitude. In this process, the $W_{f r}$ also grows with increasing $Q$ or decreasing $P$.

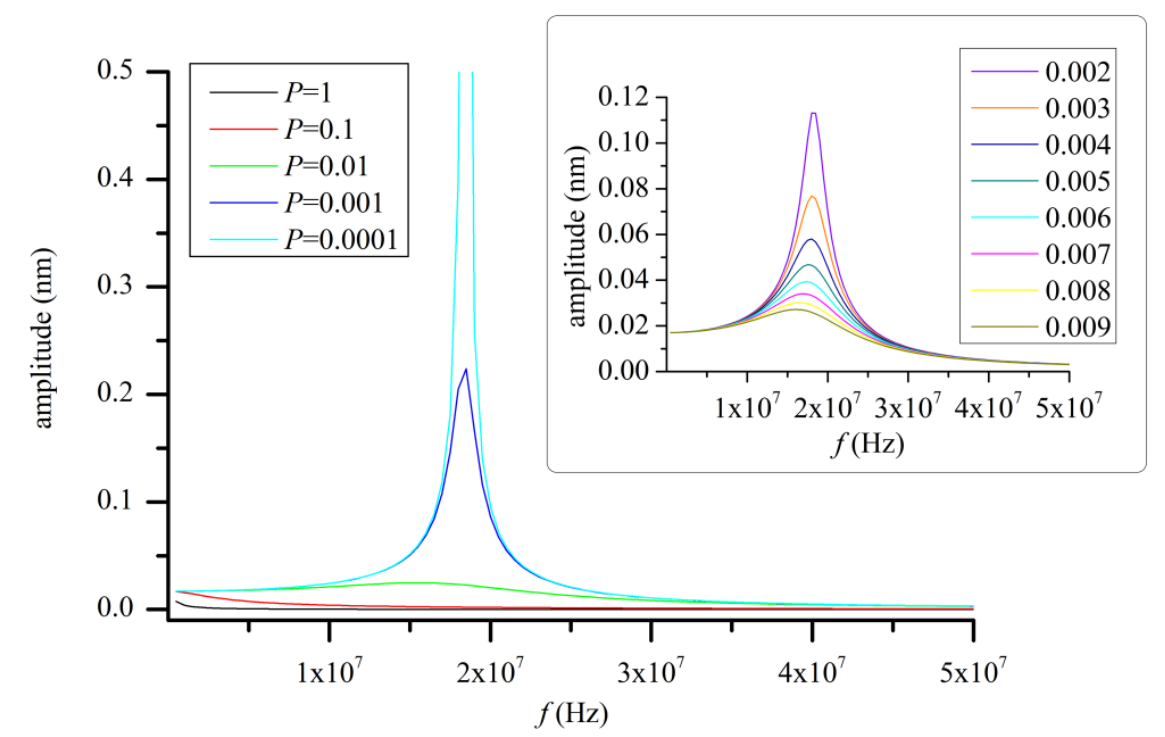

Figure 6.5 The changes in responses of damped MT vibration in cytosol due to different damping reduction factors

Here, it should be noted that the present study evaluates the reduction of cytosol damping required to stimulate prominent vibration of MTs in cells. It is however not clear whether the unique MT-cytosol interface can finally achieve the goal. Therefore in Sec. 6.3 the effect of the unique MT-cytosol interface will be further studied. In addition, the experimental evidence of MT vibration in cells is still not available in the literature. There are two possibilities, i.e., (1) The MT-cytosol interface cannot substantially decrease the damping effect of cytosol which finally quenches the vibrations of MTs in cells. (2) More advanced signal acquisition technique is 
required to identify the resonance of nanoscale components of cells and tissues. Indeed, these issues as well as many other technical challenges need to be addressed before one can eventually realise the proposed MT-based biosensors.

\subsection{Effect of nanoscale solid-liquid interface on electromechanical vibration of MTs in cytosol}

This section aims to further study the electromechanical vibration of MTs submerged in cytosol with nanoscale solid-liquid interface. The MT-cytosol interface was established in MD simulations and the electrically excited vibrations of MTs in cytosol were studied based on a MSM model. The modelling of the excitation of MT vibration in $\mathrm{EF}$, the details of the vibration analyses and the damped model used in this work can be found in Sec. 6.2.1, while the other modelling details of nanoscale MT-cytosol interface were presented in the following contents.

\subsubsection{The MD simulation and the model development}

Herein, the MD simulations were performed by using NAMD package [186]. The CHARMM22 force field was employed in the simulations, which could provide a consistent set for condensed-phase simulations of a wide variety of molecules of biological interest [192]. The cut-offs used for the non-bond interactions (vdW and electrostatic) were set to $1.2 \mathrm{~nm}$ which is proven to be applicable in protein-water system [195]. The atomic structure of tubulin $\alpha-\beta$ dimer labelled by PDB ID code 1TUB [275] was obtained in RSCB Protein Data Bank [188] and used in the present study. The dimer structure was placed at the bottom of a rectangular box where the periodic boundary conditions were applied (Fig. 6.6(a)). The box shares identical sizes in directions 1, 2 with the dimer but it was double-sized along the thickness direction of MT (direction 3). The system was solvated with TIP3P water molecules [196] to mimic the liquid environment (i.e., cell cytosol) surrounding MTs. The unbalanced negative charge was found in tubulins [274, 290, 291] and sodium-ions 
$\left(\mathrm{Na}^{+}\right)$was then added to the solution [69] to balance the charge and neutralize the whole system. The NPT ensemble [195] was used with pressure maintained at 1 atm based on the Nosé-Hoover Langevin piston method [195, 310]. Meanwhile, the temperature of the entire system was set to $310 \mathrm{~K}$ and controlled by Langevin Dynamics [195, 310]. Moreover, PME method was introduced to deal with the long-range electrostatic forces [311]. The system was energy minimized and left to equilibrate for $2520 \mathrm{ps}$ with a time step of $2 \mathrm{fs}$ [69].

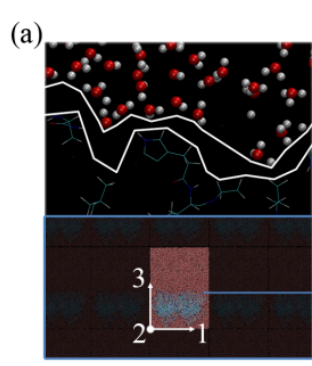

(d)

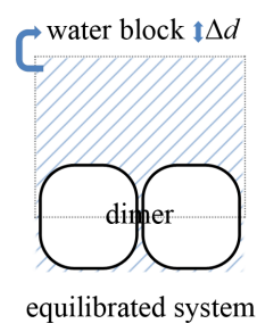

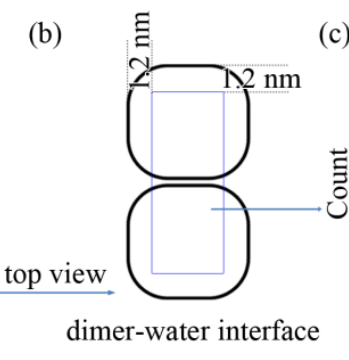

(e)

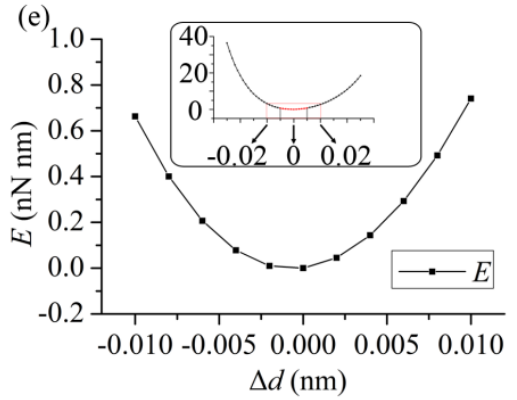

(c) 50 - $d$ between water $\&$ dimer atoms

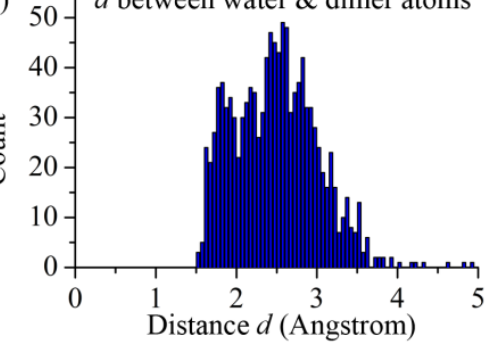

(f)

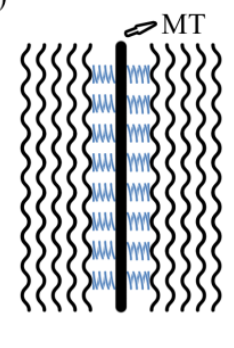

Figure 6.6 (a) The illustration of the interface between MT and cytosol (inset: simulation box); (b) the dimer region within which the distance between water molecules and dimer atoms are measured; (c) the histogram of the distances measured between the dimer and water atoms; (d) the simulations on the relation between the water-dimer distance and their interaction potential energy; (e) interface potential energy $E$ calculated as a function of the water-dimer distance change $\Delta d$; (f) the physical model for the nanoscale interface between MT and cytosol (case 3);

In the equilibrated system (Fig. 6.6(a)), a gap was found at the water-tubulin interface. The size of the gap, i.e., the distances to water were measured for dimer atoms in the central rectangular region shown in Fig. 6.6(b). The four sides of the rectangle are $1.2 \mathrm{~nm}$ (the cut-off distance for non-bond interaction) away from the corresponding boundaries of the simulation box. The boundary effect on the interface interaction is thus eliminated. Fig. 6.6(c) shows the histogram of the distance where the average value $d_{e q} 2.494$ angstroms was obtained. Subsequently, to simulate the 
interaction potential between water molecules and the dimer (Fig. 6.6(d)), the water block consisting of the water molecules on the upper half of the simulation box was manually moved up/down while the dimer was fixed at its original position. The MD simulation was performed to acquire the interface potential energy as a function of the change in the distance between the dimer and the water block that was measured by the distance the water block moves (Fig. 6.6(d)). The maximum change $\Delta d=$ $0.01 \mathrm{~nm}$ was chosen, which was reported as the amplitude of nanoscale vibrations of MT submerged in cytosol with viscous damping [282, 312]. It can be seen that the energy-distance relation is best described by a $2^{\text {nd }}$ order polynomial at $\Delta d<0.01 \mathrm{~nm}$ (Fig. 6.6(e)) or even larger deformation $\Delta d<0.02 \mathrm{~nm}$ (the inset of Fig. 6.6(e)). Thus the linear model $F_{\text {non-bond }}=-\frac{\partial E}{\partial d} \approx-k_{e q} \cdot(\Delta d)\left(k_{\mathrm{eq}}=6999 \mathrm{nN} / \mathrm{nm}\right)$ is adequate for the interaction between the dimer (or MT) and surrounding water.

The description of MT-cytosol interfaces will be introduced as follows. In the present study, two types of interfaces were investigated and compared to examine the small scale effect. The first one is the macro-scale (non-slip) interface (Fig. 6.7(a)). The requirement of continuity leads to the compatibility of the dynamic displacement along the contacting surfaces between the MT and cytosol satisfy both the MT and cytosol motions (i.e., $u_{\text {liquid }}=u_{\text {solid }}$ ) [304], which is associated with the condition that the calculated damping forces directly applied on the monomers of MT.

(a)

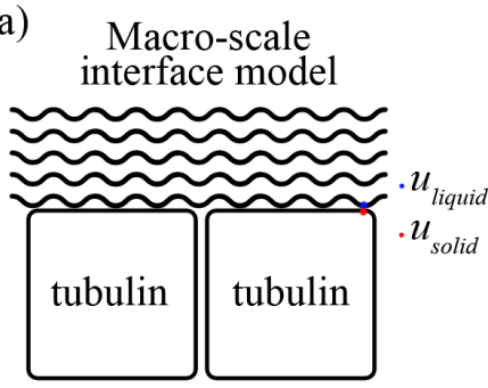

(b) Nano-scale interface model

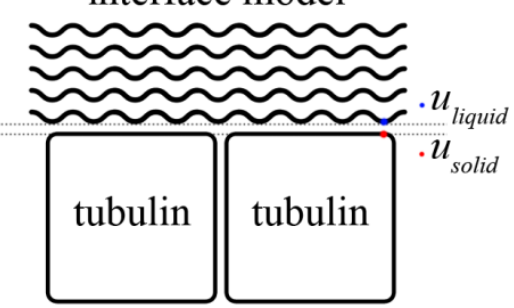

Figure 6.7 The two types of MT-cytosol interfaces models

The second case is the nanoscale interface (Fig. 6.7(b)). The displacement along the contacting surfaces between the MT and cytosol could be related by the 
description of non-bond interaction force as $F_{n o n-b o n d}=-\partial E / \partial d \approx-\left(k_{e q}\right.$. $\left.\left|u_{\text {liquid }}-u_{\text {solid }}\right|+O\left(\left(\left|u_{\text {liquid }}-u_{\text {solid }}\right|\right)^{2}\right)\right)$. Herein, $k_{e q}$ is an equivalent force coefficient to describe the force transferred between liquid and solid during the motion along the contacting surfaces due to the non-bond interactions. Thus in this condition, the calculated damping forces are transferred through the nanoscale interface to the monomers of MT.

Since the $2^{\text {nd }}$ order polynomial was the best fit to the interface energy-distance relation shown in Fig. 6.6(e) and provided that the amplitude of vibration is smaller than the value of perturbation from the equilibrium inter-phase separation distance (i.e., $\pm 0.01 \mathrm{~nm}$ ), the transfer of force due to non-bonded interaction could be achieved by a simplified model as a linear spring linking the liquid and solid at the interface.

\subsubsection{The effect of nanoscale interface on MT vibration in electric field}

To study MT vibration in cytosol, we have used the above-obtained equivalent linear springs to connect the MT with surrounding water in radial direction (Fig. 6.6(f)). The spring represents the nanoscale MT-cytosol interface (see Sec. 6.3.1) with the equivalent thickness (i.e., the natural length of the spring) $0.2494 \mathrm{~nm}$. The MT was modelled by the MSM model with the force constants for monomeric interactions updated in the studies of MD simulations [70,77, 167] and further optimized in Ref. [56]. The damping effect of the surrounding cytosol (Fig. 6.6(f)) was evaluated by the damping force $F_{d}[296,297]$ (see Sec. 6.2.1). In what follows, three different scenarios were considered: Case (1) an MT without surrounding cytosol; Case (2) an MT-cytosol system with a non-slip MT-water interface, i.e., they are in physical contact and there are no gap and no relative sliding/twisting on the MT-water interface; Case (3) an MT-cytosol system with a nanoscale MT-water interface where they are separated by a small gap and coupled with the non-bond 
interaction modelled as a linear spring. As a result, there might be sliding or twisting relative to each other on the interface. The vibrations were then excited for the MTs by an EF generated by a dipole antenna (see Sec. 6.2.1).

Fig. 6.8(a) shows that when subjected to a transverse electrical field (TEF) individual MTs (case (1)) exhibit resonant transverse vibrations at frequency 18.4 $\mathrm{MHz}$ and 78.9 MHz, respectively. They correspond to the transverse modes with one and three half waves. The bandwidth of the $1^{\text {st }}$ peak is found to be several times wider than that of the $2^{\text {nd }}$ peak. The results for MTs submerged in cytosol (i.e., cases (2) and (3)) are shown in Fig. 6.8(b) where the damping effect of cytosol is of major concern $[58,278,283,300]$. It is noted that, due to the damping effect the amplitude decreased monotonically with the increasing frequency and at least an order of magnitude lower than those of individual MT (Fig. 6.8(a)).

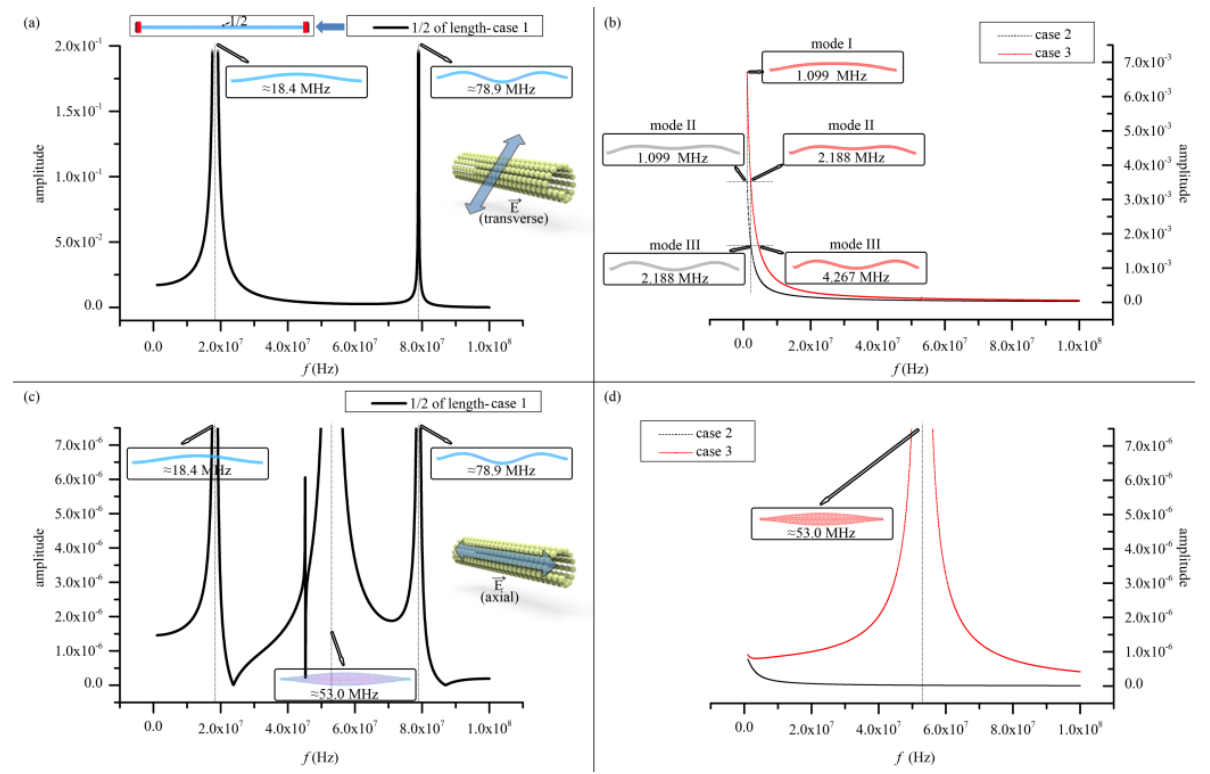

Figure 6.8 The amplitude-frequency spectrum of (a) individual MT (case 1) subject to a TEF, (b) MT-cytosol system subject to a TEF with a non-slip (case (2)) or a nanoscale interface (case (3)), (c) individual MT subject to an AEF and (d) MT-cytosol system subject to an AEF with a non-slip (case (2)) or a nanoscale interface (case (3)).

In the present study, the primary goal is to examine the effect of the nanoscale MT-cytosol interface on the vibrations of MTs in cytosol, i.e., the difference between cases (2) and (3). The frequency of transverse mode II in Fig. 6.8(b) is found to 
increase from $1.099 \mathrm{MHz}$ for case (2) to $2.188 \mathrm{MHz}$ for case (3). Similar trend is also observed for mode III in Fig. 6.8(b). In addition, at a given frequency the amplitude growth of case (3) relative to case (2) ranges from $26.1 \%$ to $146.5 \%$ in the frequency range $[1 \mathrm{MHz}, 100 \mathrm{MHz}]$. It follows that the nanoscale interface (case (3)) upshifts the frequency of the transverse vibration and raises the vibration amplitude at a given frequency. These can be attributed to the presence of the nano-scale interface, where the slip boundary or the gap between MT and cytosol will largely decrease the energy dissipation caused by the cytosol damping.

Fig. 6.8(c) shows the spectrum of MT vibration (case (1)) excited by an axial $\mathrm{EF}$ (AEF). It is found that an AEF generates so-called radial breathing vibration (RBV) of an MT with the associated frequency around $53 \mathrm{MHz}$. In RBV, the $\alpha$ and $\beta$ tubulins of the MT oscillate in the radial direction as if the MT was breathing. On an MT cross-section, the time-dependent change in radius (i.e., radial vibration displacement) is equal in all directions and its amplitude (Fig. 6.8(c)) is found to be 3 orders of magnitudes smaller than those of transverse MT vibrations. The vibration spectra of RBV were calculated in Fig. 6.8(d) for MT-water system with the nanoscale interface (case (3)) in comparison with those for the non-slip interface (case (2)). In case (2), the vibration amplitude decreases continuously with rising frequency. This behaviour is analogous to the transverse vibration of MTs in cytosol (Fig. 6.8(b)) and the vibration of a bulk solid object submerged in water. In sharp contrast, the frequency spectrum in case (3) shows a resonant vibration where the amplitude rises suddenly when the frequency approaches $53 \mathrm{MHz}$, i.e., the resonant RBV frequency. This is also observed in the spectrum of the free RBV (Fig. 6.8(c)). These results indicate that the nanoscale interface can largely decrease the cytosol damping and lead to the resonant RBV of MT in cytosol (Fig. 6.8(d)-case (3)).

To further examine the effect of the nanoscale interface gap, we examined the trend of the amplitude to change with time (at a given frequency) for the MTs in all three cases considered. The forced MT vibration was excited by an EF and lasted for 
9.75 periods $(T)$ (i.e., stage I). Subsequently, the excitation was removed and the MT vibrated freely (i.e., stage II) in cytosol. The time-dependent amplitude at the two stages is presented in Fig. 6.9 with three excitation frequencies, i.e., $1 \mathrm{MHz}, 18 \mathrm{MHz}$ (i.e., the resonant transverse vibration frequency) and $53 \mathrm{MHz}$ (the resonant $\mathrm{RBV}$ frequency). It is noted in Fig. 6.9(a), achieved by applying the TEF of $1 \mathrm{MHz}$, that an MT in case (1) showed nearly constant amplitudes at both stages I and II, and the amplitude at stage I is around 2 times greater than the one at stage II. When the cytosol was introduced, the MT of case (2) vibrates steadily at stage I but the vibration extinguishes very quickly at stage II (Fig. 6.9(a)). The trend obtained for the MT in case (3) is close to that of the MT in cases (2). The difference is that, in case (3) the MT shows greater amplitude at stage I due to the reduced damping effect in the presence of the nanoscale interface gap. For the transverse vibration at resonant frequency $18 \mathrm{MHz}$, the amplitude achieved in Fig. 6.9(b) for MTs in case (1) grows constantly at stage I and finally reaches its maximum value, which remains unchanged throughout stage II. The inset of Fig. 6.9(b) shows how the amplitude of MTs changes in cases (2) and (3), which are similar to those shown in Fig. 6.9(a). These results indicates that the free transverse MT vibration cannot survive the cytosol damping even if the nanoscale interface gap (in case (3)) is considered.

Next, the RBV of MTs excited by AEF was studied in Fig. 6.9(c-d) obtained at frequency $1 \mathrm{MHz}$ and the resonant frequency $53 \mathrm{MHz}$, respectively. It is noted in the two figures that, at the two frequencies RBV is again quenched by cytosol (i.e., the amplitude fades away with time in a short period of time) at stage II when the non-slip MT-cytosol interface (case (2)) was assumed (the insets of Fig. 6.9(c-d)). Nevertheless, when the nanoscale MT-cytosol interface gap was introduced (i.e., case (3)) the amplitude of MT remains constant or nearly undamped at stage II (Fig. 6.9(c-d)), which is nearly the same as the behaviour of free RBV in case (1). At $1 \mathrm{MHz}$ (Fig. 6.9(c)) the amplitude in case (3) is smaller than the one in case (1) while, at resonant frequency $53 \mathrm{MHz}$ (Fig. 6.9(d)) the amplitudes in the two cases are nearly 
the same. Based on the results, we came to the conclusion that the influence of the nanoscale MT-cytosol interface gap is so great that it can nearly eliminate the effect of cytosol damping on the RBV of MT. Such influence turns out to be even stronger for the resonant RBV of the MT submerged in cytosol. The RBV of the MT with high frequency and small amplitude (i.e., < $0.1 \AA$, which is more than 1000 times smaller than those in TEF) thus is under the protection of the nanoscale solid-liquid interface gap and is promising for developing MT-based biosensor in cells. In this

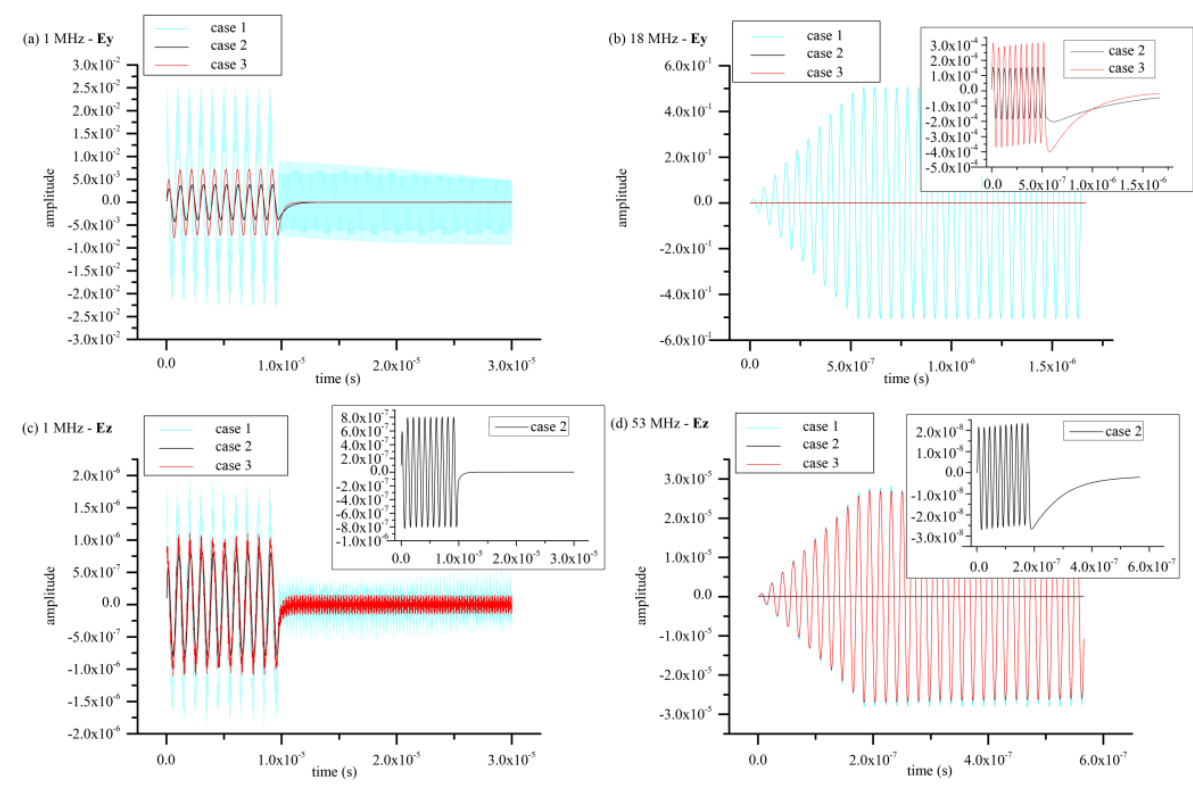

Figure 6.9 Time-dependent amplitude of MT vibration excited by a TEF at frequency (a) $1 \mathrm{MHz}$ and (b) $18 \mathrm{MHz}$, respectively, and the vibration stimulated by an AEF at (c) $1 \mathrm{MHz}$ and (d) $53 \mathrm{MHz}$, respectively.

case, there existed the conversion between the potential energy of the nanoscale interface gap and kinetic energy of oscillating MT but total energy is nearly conserved with almost no energy dissipation via the motion of surrounding water. This can be attributed to the fact that due to the small amplitude of RBV the force on the surrounding water from the oscillating MT is not large enough to generate the radial motion of the water. In other words, the water behaves like a rigid body with almost zero displacement and velocity. Accordingly, cytosol damping or the energy dissipation via cytosol is negligible. 


\subsection{Summary}

In this chapter, firstly, an alternating EF was used to excite the forced vibration of polarized MTs in different modes. The possibility was also examined to detect the abnormal tubulin interaction in the pathological process by measuring the changes in the frequency and elastic moduli of MTs. The effect of cytosol damping was also preliminarily evaluated for the transverse vibration of the MT.

It is found that a TEF is able to excite transverse vibrations of MTs where frequency upshifts from 18.4 to $240.3 \mathrm{MHz}$ when the half axial wavenumber rises from one to seven. The vibrations with the even half wavenumbers are hard to excite as they show a very narrow $W_{f r}$ on the vibration spectra, while it is relatively easy to achieve those with odd half wavenumber due to their much wider $W_{f r}$ which increases with the decreasing frequency. An axial EF is found to generate RBMs of MTs, which can be observed in the vibration spectra at a frequency around 53.019 $\mathrm{MHz}$ and $159.291 \mathrm{MHz}$, much higher than the frequencies of the transverse vibrations with the same half axial wavenumber. The circumferential vibrations with non-circular cross-section were also achieved at 585.639 MHz. In addition, the axial vibration of frequency $377.840 \mathrm{MHz}$ can also be excited, where the amplitude was found to be greater than other modes stimulated by an axial EF.

For MTs in cells, the excitation of prominent vibrations depends largely on the possible MT-cytosol sliding at the interface, which may substantially reduce cytosol damping. The transverse vibration with amplitude greater than $0.1 \mathrm{~nm}$ and quality factor $Q$ larger than 4.3 can be achieved by applying an EF of $20 \mathrm{~V} / \mathrm{m}$ provided that the sliding MT-cytosol interface can largely decrease the damping by three orders of magnitude relative to the cytosol damping due to a normal continuous (non-slip) MT-cytosol interface. In addition, it was also found that the longitudinal tubulin interaction determines the axial Young's modulus that controls the bending deformation or the transverse vibration, while the helical tubulin interaction mainly decides shear modulus and the inter-PF sliding. Thus, changes in the longitudinal or 
helical interaction in pathological processes can be detected via the variation of elastic moduli and the shift of MT frequency as promising biomarkers. Indeed, correlating the pathological changes of MTs to their responses to an alternating EF is crucial for the development of MT-based biosensors. Hence, the dependence of MT frequency on the tubulin interaction achieved here not only reveal the structure-property relation of MTs, but also provides useful guidance to the development of the MT-based biosensors to detect the changes in the mechanical properties and structure of individual MTs or cells.

Furthermore, MD simulations and MSM simulations were performed to study the effect of the interface on the electrically excited vibration of MTs in cytosol. It was found that a forced transverse vibration of MTs can be excited by a TEF whose amplitude decreases with the rising excitation frequency. The vdW interaction between MT and cytosol significantly raises the frequency of a vibration mode and lifts the amplitude at a given frequency. It however does not give rise to a resonant transverse vibration of MTs in cytosol. In addition, the free transverse vibration of MTs will be wiped out very quickly by cytosol damping even if the nanoscale interface gap is considered. Forced RBV of MTs can be stimulated by an AEF. Similar to the transverse vibration in cytosol, no resonant RBV can be achieved when the non-slip MT-cytosol interface is assumed. The nanoscale interface gap, on the other hand, largely decreases the cytosol damping and results in resonant RBV of MTs in cytosol. The amplitude of RBV and thus the MT-cytosol interaction becomes very small. In this case, the surrounding water behaves as if it were a rigid body with nearly zero displacement and velocity. This finally leads to an almost undamped free RBV of MTs in cytosol. These results provide important guidance for the development of the inherent and bio-compatible nano-biosensors based on MTs in cells that would enable non-invasive diagnosis of disease and health monitoring of cells and tissues. Such a resonant RBV of MTs excited by an electrical field may also have a role in signal transduction in cells. 


\section{Chapter 7 Conclusions}

\subsection{Conclusive remarks}

The CSK is a subcellular structure responsible for cell shape and intercellular organization. The mechanical support provided by CSK plays a key role in fulfilling the essential functions of cells, such as division and movement. The CSK is a combination of several different subcellular components. The basic subcellular components are MTs, F-actins and IFs. The pivotal roles played by CSK depend crucially on the mechanical responses/properties of CSK filaments. Thus, an in-depth understanding of CSK filament mechanics is essential in revealing how cells fulfil their biological functions via CSK and offering the new design of biomimetic structure/materials by mimicking the mechanical characteristics of CSK filaments. The experimental works have been conducted initially to study the mechanics of CSK filaments. In addition, the effort has also been devoted to theoretically characterising the mechanics of those filaments. In spite of the results obtained in the existing modelling studies, challenges still exist in the mechanical modelling of CSK filaments, which necessitate further investigation on the mechanics of CSK. More importantly, in facilitating to develop the CSK filament-based biosensor and biomimetic nanomaterials, it is crucial to obtain deeper understanding in inherent (e.g., monomer interactions, helical structure) and environmental factors (e.g., surrounding proteins, cytosol, physical field) which determine the nanomechanics of CSK filaments. Thus in this thesis simulations have been performed to study the monomeric features of micro-scale CSK filaments, the effect of unique helical structure on CSK filaments mechanics, the influence of filamentous surrounding environment on individual CSK filament and the interaction between the external physical field and the filaments, etc.

A comprehensive review of the great amount of efforts into modelling of CSK filaments was first provided in the thesis. The review is devoted to summarise and 
classify a wealth of computational models for CSK filaments, with concentrations on their purposes and applications. The comprehensive summary achieved in the present thesis is thus able to shed some lights on the development of the integrated researches and the potential applications in medical innovations.

MSM models developed for MTs and F-actin were then introduced, which are capable of interpreting monomeric features of CSK filaments in a relatively large scale and with high computational efficiency. The MSM model was employed to identify the origin of the inter-PF sliding and its role in bending and vibration of MTs. Clear evidence shows that the inter-PF sliding is due to the soft inter-PF bonds and leads to the length-dependent bending stiffness. In addition, the Euler beam theory is found to adequately describe MT deformation when the inter-PF sliding is largely prohibited. Nevertheless, neither shear deformation nor the nonlocal effect considered in the 'more accurate' beam theories can fully capture the effect of the inter-PF sliding. The results achieved here reflect the distinct deformation mechanisms between an MT (which is composed of discrete basic units) and its equivalent continuous body.

In studying helix structure effects on the mechanics of CSK filaments, a 3D transverse vibration was reported for MTs, where the bending axis of the cross section rotates in an anticlockwise direction and the adjacent half-waves oscillate in different planes. A close correlation was confirmed between the rotation of the oscillation planes and the helical structures of MTs, showing that the 3D mode is a result of the helicity found in MTs. In addition, the unique vibration mode is found to be a result of the bending axis rotation of the cross-section but no significant torsion occurs for MTs.

The tension-induced bending was studied for F-actins as a result of their helical structure. The study shows that the helical structure of F-actin leads to a resultant eccentric force and thus, a resulted bending moment on the cross-section of the F-actin when an axial tension/compression is applied. For a given axial load the 
induced bending deflection of F-actin increases substantially with the rising contour length but decreases slightly with growing rotation angle.

After that, subcellular environment effect on the filament mechanics was explored. In cells, the protein cross-linkers lead to the distinct buckling behaviour of MTs different from the buckling of individual MTs. The transition of buckling responses was captured as the $2 \mathrm{D}$-linkers were replaced by the $3 \mathrm{D}$ ones. It was shown that more uniform distribution of the radial orientation of the 3D-linkers leads to the higher critical load. The inhomogeneity of the axial density results in the localized buckling patterns, which reveals the physical origin of the experimentally observed localized buckling.

Moreover, structural instability was investigated for the F-actins in filopodial protrusion by considering the reinforcing effect of the ABPs. The predicted buckling load agrees well with the experimentally obtained stall force, showing a pivotal role of the ABPs in regulating the stiffness of F-actin bundles during the formation of filopodia protrusion. The outcomes in this study reveal the role played by cross-linking proteins in CSK mechanics and the difference in mechanical aspect between the individual filaments and the filaments in CSK.

Finally, the electromechanical vibration of CSK filaments was explored, which may have an important role in designing inherent biosensors. Here, transverse vibration, radial breathing vibration and axial vibration were achieved for MTs subject to a transverse or an axial EF. The strong correlation obtained between the tubulin interaction and MT vibration excited by EF provides a new avenue to a non-contacting technique for the structural or property changes in MTs, where frequency shift is used as a biomarker. This technique can be used for individual MTs and is possible for those in cells when the cytosol damping on MT vibrations is largely reduced by the unique features of MT-cytosol interface.

Subsequently, the unique features of nanoscale MT-cytosol interface were studied with MD and MSM simulations. The simulations show that the solid-liquid 
interface with a nanoscale gap significantly reduces the viscous damping of cytosol on MT vibration. Specifically, as far as the RBMs were concerned, cytosol behaves as a rigid body and thus, has negligible damping effect on the RBM of MTs. This distinctive feature of the RBMs arises from its extremely small amplitude $(<0.1 \AA)$ and relatively large gap between MT and cytosol (2.5 $\AA)$ due to the vdW interaction. Contributed by the EF excited megahertz MT vibration achieved in this study, the potential roles of CSK filaments in designing MT-based biosensors, developing novel treatments of diseases and facilitating signal transduction in cells were disclosed.

\subsection{Future work}

The existing works (including the present study) have explored numerous aspects in the topic of CSK mechanics and achieved a considerable amount of outcomes. However, there are still countless challenges to overcome in deepening the fundamental understanding of the physics and mechanics of CSK filaments in living cell. Those crucial fundamental understanding could help researchers to design novel diagnostic tools, to develop novel treatments of diseases and to understand the mysterious aspects of life (e.g., the nature of consciousness). Not only the experimental technologies need to be improved, but also more advanced features are necessary to be included in the future models.

At the next stage, the most anticipated breakthrough is building a 'cell scale' model with different cytoskeletal components included and enabling it to interact with external EF. Meanwhile, a corresponding experiment could be conducted to compare with the simulations to figure out the roles of cytoskeletal components in the interaction between EF and cell.

Besides this anticipation, there are also many other aspects that need to be addressed in future works to serve as the following researches of the present thesis. The first thing is to improve the MSM model to endow it with the ability to flexibly 
characterise different loading modes in various environmental conditions. For instance, the force constants could be optimized for different loading situations and validated through the experimental data. Then, as far as a case with non-linear large deformation is considered, the non-linear feature could be achieved through various optimizations on the model. For example, the strain-dependent constants for basic elements, the additional structural elements between the two nodes of a beam in MSM model to represent the nonlinear feature of monomer interaction, etc. Also it is worth mentioning that since the nano-filaments are influenced by the heat in the environment, it is necessary to included heat induced strain in the model.

The second progress could be achieved in exploring the influence of pathological changes due to diseases on the CSK filaments/components mechanics. At nanoscale, the future model is expected to reveal the monomeric changes in filaments due to pathological process. Then with an integrated model of multiple filaments, the consequences of the disease on multi-filament components could be disclosed. Further improving the spatial scale of the model, one could explore the effect of pathological changes on cell with information from the nanoscale of cell. With proper comparisons by experiments, it is expected that the findings could help develop novel treatments of diseases.

Another aspect that needs to be addressed in the future is the mechanotransduction through CSK filaments. The regulation of various cell functions by mechanical forces has by now been amply demonstrated. Because all cells of an organism are exposed to external forces, it is confirmed that cells could sense these forces. Subsequently, these mechanical forces are known to cause changes in intracellular biochemistry and gene expression. Thus, it is expected a random CSK network model could be coupled with other cellular components such as plasma membrane or nucleus membrane. Thus the model could be applied on investigations such as the transmission of mechanical signal from external environment to the centre of the cell. With the obtained results, the mechanism of 
cell response to external mechanical signal could be revealed.

Finally, it is highly expected that the data acquisition technology in relevant experiments could be advanced to characterise the detailed nanoscale components behaviours in a living cell. 


\section{References}

[1] Rodriguez, M.L., McGarry, P.J. \& Sniadecki, N.J. 2013 Review on cell mechanics: experimental and modeling approaches. Applied Mechanics Reviews $\mathbf{6 5}$, 060801.

[2] O’Connor, C.M., Adams, J.U. \& Fairman, J. 2010 Essentials of cell biology. Cambridge, MA: NPG Education 1.

[3] Pollard, T.D.T.D. \& Goldman, R.D. 2017 The cytoskeleton, Cold Spring Harbor, New York Cold Spring Harbor Laboratory Press.

[4] Fletcher, D.A. \& Mullins, R.D. 2010 Cell mechanics and the cytoskeleton. Nature 463, 485-492.

[5] Dobbie, I., Linari, M., Piazzesi, G. \& Reconditi, M. 1998 Elastic bending and active tilting of myosin heads during muscle contraction. Nature 396, 383.

[6] Geeves, M.A. 1991 The dynamics of actin and myosin association and the crossbridge model of muscle contraction. Biochemical Journal 274, 1.

[7] Linari, M., Brunello, E., Reconditi, M., Fusi, L., Caremani, M., Narayanan, T., Piazzesi, G., Lombardi, V. \& Irving, M. 2015 Force generation by skeletal muscle is controlled by mechanosensing in myosin filaments. Nature 528, 276-279.

[8] Goldstein, L.S. \& Yang, Z. 2000 Microtubule-based transport systems in neurons: the roles of kinesins and dyneins. Annual Review of Neuroscience 23, 39-71.

[9] Hatzfeld, M., Keil, R. \& Magin, T.M. 2017 Desmosomes and intermediate filaments: their consequences for tissue mechanics. Cold Spring Harbor Perspectives in Biology 9, a029157.

[10] Mak, M., Kim, T., Zaman, M.H. \& Kamm, R.D. 2015 Multiscale mechanobiology: computational models for integrating molecules to multicellular systems. Integrative Biology 7, 1093-1108.

[11] Gittes, F., Mickey, B., Nettleton, J. \& Howard, J. 1993 Flexural rigidity of microtubules and actin filaments measured from thermal fluctuations in shape. Journal of Cell Biology 120, 923-934. 
[12] Fujime, S. 1970 Quasi-elastic light scattering from solutions of macromolecules.

II. Doppler broadening of light scattered from solutions of semi-flexible polymers, F-actin. Journal of the Physical Society of Japan 29, 751-759.

[13] Nagashima, H. \& Asakura, S. 1980 Dark-field light microscopic study of the flexibility of F-actin complexes. Journal of Molecular Biology 136, 169-182.

[14] Takebayashi, T., Morita, Y. \& Oosawa, F. 1977 Electronmicroscopic investigation of the flexibility of F-actin. Biochimica et Biophysica Acta (BBA)-Protein Structure 492, 357-363.

[15] Isambert, H., Venier, P., Maggs, A.C., Fattoum, A., Kassab, R., Pantaloni, D. \& Carlier, M.-F. 1995 Flexibility of actin filaments derived from thermal fluctuations. Effect of bound nucleotide, phalloidin, and muscle regulatory proteins. Journal of Biological Chemistry 270, 11437-11444.

[16] Yoshino, S., Umazume, Y., Natori, R., Fujime, S. \& Chiba, S. 1978 Optical diffraction study of muscle fibers: II. Electro-optical properties of muscle fibers. Biophysical Chemistry 8, 317-326.

[17] Yanagida, T. \& Oosawa, F. 1978 Polarized fluorescence from $\varepsilon$-ADP incorporated into F-actin in a myosin-free single fiber: Conformation of F-actin and hanges induced in it by heavy meromyosin. Journal of Molecular Biology 126, 507-524.

[18] Yasuda, R., Miyata, H. \& Kinosita Jr, K. 1996 Direct measurement of the torsional rigidity of single actin filaments. Journal of Molecular Biology 263, 227-236.

[19] Simmonst, R., Finer, J., Warrick, H., Kralik, B., Chu, S. \& Spudich, J. 1993 Force on single actin filaments in a motility assay measured with an optical trap. In Mechanism of Myofilament Sliding in Muscle Contraction (pp. 331-337, Springer.)

[20] Pampaloni, F., Lattanzi, G., Jonas, A., Surrey, T., Frey, E. \& Florin, E.L. 2006 Thermal fluctuations of grafted microtubules provide evidence of a length-dependent persistence length. Proceedings of the National Academy of Sciences of the United 
States of America 103, 10248-10253.

[21] Kis, A., Kasas, S., Babić, B., Kulik, A., Benoit, W., Briggs, G., Schönenberger, C., Catsicas, S. \& Forro, L. 2002 Nanomechanics of microtubules. Physical Review Letters 89, 248101.

[22] Chugh, P., Clark, A.G., Smith, M.B., Cassani, D.A., Dierkes, K., Ragab, A., Roux, P.P., Charras, G., Salbreux, G. \& Paluch, E.K. 2017 Actin cortex architecture regulates cell surface tension. Nature Cell Biology 19, 689.

[23] Hu, L., Su, P., Li, R., Yan, K., Chen, Z., Shang, P. \& Qian, A. 2015 Knockdown of microtubule actin crosslinking factor 1 inhibits cell proliferation in MC3T3-E1 osteoblastic cells. BMB reports $\mathbf{4 8}, 583$.

[24] Lazarides, E. 1976 Actin, alpha-actinin, and tropomyosin interaction in the structural organization of actin filaments in nonmuscle cells. The Journal of cell biology 68, 202-219.

[25] Bidone, T.C., Kim, T., Deriu, M.A., Morbiducci, U. \& Kamm, R.D. 2015 Multiscale impact of nucleotides and cations on the conformational equilibrium, elasticity and rheology of actin filaments and crosslinked networks. Biomechanics and modeling in mechanobiology 14, 1143-1155.

[26] Oda, T., Iwasa, M., Aihara, T., Maéda, Y. \& Narita, A. 2009 The nature of the globular-to fibrous-actin transition. Nature 457, 441-445.

[27] Peskin, C.S., Odell, G.M. \& Oster, G.F. 1993 Cellular motions and thermal fluctuations: the Brownian ratchet. Biophysical Journal 65, 316-324.

[28] Mogilner, A. \& Oster, G. 1996 Cell motility driven by actin polymerization. Biophysical Journal 71, 3030-3045.

[29] Mogilner, A. \& Oster, G. 1996 The physics of lamellipodial protrusion. European Biophysics Journal 25, 47-53.

[30] Dickinson, R.B., Caro, L. \& Purich, D.L. 2004 Force generation by cytoskeletal filament end-tracking proteins. Biophysical Journal 87, 2838-2854.

[31] ben-Avraham, D. \& Tirion, M.M. 1995 Dynamic and elastic properties of 
F-actin: a normal-modes analysis. Biophysical Journal 68, 1231-1245.

[32] Everaers, R., Jülicher, F., Ajdari, A. \& Maggs, A.C. 1999 Dynamic Fluctuations of Semiflexible Filaments. Physical Review Letters 82, 3717-3720.

[33] Wilhelm, J. \& Frey, E. 1996 Radial distribution function of semiflexible polymers. Physical Review Letters 77, 2581.

[34] Sept, D. \& McCammon, J.A. 2001 Thermodynamics and kinetics of actin filament nucleation. Biophysical Journal 81, 667-674.

[35] Inoue, Y., Deji, T., Shimada, Y., Hojo, M. \& Adachi, T. 2010 Simulations of dynamics of actin filaments by remodeling them in shearflows. Computers in Biology and Medicine 40, 876-882.

[36] Chu, J.-W. \& Voth, G.A. 2006 Coarse-grained modeling of the actin filament derived from atomistic-scale simulations. Biophysical Journal 90, 1572-1582.

[37] Wang, C.Y., Ru, C.Q. \& Mioduchowski, A. 2006 Vibration of microtubules as orthotropic elastic shells. Physica E: Low-dimensional Systems and Nanostructures 35, 48-56.

[38] Qin, Z., Kreplak, L. \& Buehler, M.J. 2009 Hierarchical structure controls nanomechanical properties of vimentin intermediate filaments. PloS One 4, e7294.

[39] Ming, D., Kong, Y., Wu, Y. \& Ma, J. 2003 Substructure synthesis method for simulating large molecular complexes. Proceedings of the National Academy of Sciences 100, 104-109.

[40] Ming, D., Kong, Y., Wu, Y. \& Ma, J. 2003 Simulation of F-actin filaments of several microns. Biophysical Journal 85, 27-35.

[41] Chu, J.-W. \& Voth, G.A. 2005 Allostery of actin filaments: molecular dynamics simulations and coarse-grained analysis. Proceedings of the National Academy of Sciences of the United States of America 102, 13111-13116.

[42] Matsushita, S., Adachi, T., Inoue, Y., Hojo, M. \& Sokabe, M. 2010 Evaluation of extensional and torsional stiffness of single actin filaments by molecular dynamics analysis. Journal of Biomechanics 43, 3162-3167. 
[43] Deriu, M.A., Bidone, T.C., Mastrangelo, F., Di Benedetto, G., Soncini, M., Montevecchi, F.M. \& Morbiducci, U. 2011 Biomechanics of actin filaments: a computational multi-level study. Journal of Biomechanics 44, 630-636.

[44] Fan, J., Saunders, M.G. \& Voth, G.A. 2012 Coarse-graining provides insights on the essential nature of heterogeneity in actin filaments. Biophysical Journal 103, 1334-1342.

[45] Yogurtcu, O.N., Kim, J.S. \& Sun, S.X. 2012 A mechanochemical model of actin filaments. Biophysical Journal 103, 719-727.

[46] Fan, J., Saunders, M.G., Haddadian, E.J., Freed, K.F., Enrique, M. \& Voth, G.A. 2013 Molecular origins of cofilin-linked changes in actin filament mechanics. Journal of Molecular Biology 425, 1225-1240.

[47] Yamaoka, H. \& Adachi, T. 2010 Coupling between axial stretch and bending/twisting deformation of actin filaments caused by a mismatched centroid from the center axis. International Journal of Mechanical Sciences 52, 329-333.

[48] Li, S., Zhang, J., Wang, C. \& Nithiarasu, P. 2018 Atomistic Modeling of F-Actin Mechanical Responses and Determination of Mechanical Properties. ACS Biomaterials Science \& Engineering 4, 2794-2803.

[49] Chrétien, D. \& Fuller, S.D. 2000 Microtubules switch occasionally into unfavorable configurations during elongation. Journal of Molecular Biology 298, 663-676.

[50] Howard, J. \& Hyman, A.A. 2003 Dynamics and mechanics of the microtubule plus end. Nature 422, 753-758.

[51] Dogterom, M. \& Yurke, B. 1997 Measurement of the force-velocity relation for growing microtubules. Science 278, 856-860.

[52] Mogilner, A. \& Oster, G. 1999 The polymerization ratchet model explains the force-velocity relation for growing microtubules. European Biophysics Journal 28, 235-242.

[53] Chrétien, D., Flyvbjerg, H. \& Fuller, S.D. 1998 Limited flexibility of the 
inter-protofilament bonds in microtubules assembled from pure tubulin. European Biophysics Journal 27, 490-500.

[54] Chretien, D. \& Wade, R.H. 1991 New data on the microtubule surface lattice. Biologie Cellulaire 71, 161-174.

[55] Portet, S., Tuszyński, J., Hogue, C. \& Dixon, J. 2005 Elastic vibrations in seamless microtubules. European Biophysics Journal 34, 912-920.

[56] Li, S., Wang, C. \& Nithiarasu, P. 2018 Structure-property relation and relevance of beam theories for microtubules: a coupled molecular and continuum mechanics study. Biomechanics and modeling in mechanobiology 17, 339-349.

[57] Sirenko, Y.M., Stroscio, M.A. \& Kim, K. 1996 Elastic vibrations of microtubules in a fluid. Physical Review E 53, 1003.

[58] Wang, C.Y., Li, C.F. \& Adhikari, S. 2009 Dynamic behaviors of microtubules in cytosol. Journal of Biomechanics 42, 1270-1274.

[59] Wang, C.Y., Ru, C.Q. \& Mioduchowski, A. 2006 Orthotropic elastic shell model for buckling of microtubules. Physical Review. E: Statistical, Nonlinear, and Soft Matter Physics 74, 052901.

[60] Wang, C.Y. \& Zhang, L.C. 2008 Circumferential vibration of microtubules with long axial wavelength. Journal of Biomechanics 41, 1892-1896.

[61] Li, C., Ru, C.Q. \& Mioduchowski, A. 2006 Length-dependence of flexural rigidity as a result of anisotropic elastic properties of microtubules. Biochemical and Biophysical Research Communications 349, 1145-1150.

[62] Shi, Y.J., Guo, W.L. \& Ru, C.Q. 2008 Relevance of Timoshenko-beam model to microtubules of low shear modulus. Physica E: Low-dimensional Systems and Nanostructures 41, 213-219.

[63] Gao, Y.W. \& Lei, F.-M. 2009 Small scale effects on the mechanical behaviors of protein microtubules based on the nonlocal elasticity theory. Biochemical and Biophysical Research Communications 387, 467-471.

[64] Li, T. 2008 A mechanics model of microtubule buckling in living cells. Journal 
of Biomechanics 41, 1722-1729.

[65] Kasas, S., Kis, A., Riederer, B.M., Forró, L., Dietler, G. \& Catsicas, S. 2004 Mechanical properties of microtubules explored using the finite elements method. Chemphyschem 5, 252-257.

[66] Civalek, Ö. \& Demir, C. 2016 A simple mathematical model of microtubules surrounded by an elastic matrix by nonlocal finite element method. Applied Mathematics and Computation 289, 335-352.

[67] Daneshmand, F., Farokhi, H. \& Amabili, M. 2014 A higher-order mathematical modeling for dynamic behavior of protein microtubule shell structures including shear deformation and small-scale effects. Mathematical Biosciences 252, 67-82.

[68] Mehrbod, M. \& Mofrad, M.R. 2011 On the significance of microtubule flexural behavior in cytoskeletal mechanics. PloS One 6, e25627.

[69] Deriu, M.A., Enemark, S., Soncini, M., Montevecchi, F.M. \& Redaelli, A. 2007 Tubulin: from atomistic structure to supramolecular mechanical properties. Journal of Materials Science 42, 8864-8872.

[70] Ji, X.Y. \& Feng, X.Q. 2011 Coarse-grained mechanochemical model for simulating the dynamic behavior of microtubules. Physical Review E 84, 031933.

[71] Ji, X.Y. \& Feng, X.Q. 2011 Mechanochemical modeling of dynamic microtubule growth involving sheet-to-tube transition. PloS One 6, e29049.

[72] Deriu, M.A., Soncini, M., Orsi, M., Patel, M., Essex, J.W., Montevecchi, F.M. \& Redaelli, A. 2010 Anisotropic elastic network modeling of entire microtubules. Biophysical Journal 99, 2190-2199.

[73] Taute, K.M., Pampaloni, F., Frey, E. \& Florin, E.-L. 2008 Microtubule dynamics depart from the wormlike chain model. Physical Review Letters 100, 028102.

[74] Heussinger, C., Schüller, F. \& Frey, E. 2010 Statics and dynamics of the wormlike bundle model. Physical Review E 81, 021904.

[75] Xiang, P. \& Liew, K.M. 2012 Free vibration analysis of microtubules based on an atomistic-continuum model. Journal of Sound and Vibration 331, 213-230. 
[76] Xiang, P. \& Liew, K.M. 2011 Predicting buckling behavior of microtubules based on an atomistic-continuum model. International Journal of Solids and Structures 48, 1730-1737.

[77] Zhang, J. \& Wang, C.Y. 2014 Molecular structural mechanics model for the mechanical properties of microtubules. Biomechanics and modeling in mechanobiology 13, 1175-1184.

[78] Zhang, J. \& Wang, C.Y. 2016 Free vibration analysis of microtubules based on the molecular mechanics and continuum beam theory. Biomechanics and modeling in mechanobiology 15, 1069-1078.

[79] Zhang, J. \& Wang, C. 2017 Boundary condition-selective length dependence of the flexural rigidity of microtubules. Physics Letters A.

[80] Li, S., Wang, C.Y. \& Nithiarasu, P. 2017 Three-dimensional transverse vibration of microtubules. Journal of Applied Physics 121, 234301.

[81] Li, S., Wang, C. \& Nithiarasu, P. 2018 Effects of the cross-linkers on the buckling of microtubules in cells. Journal of Biomechanics 72, 167-172.

[82] Wang, C.Y., Guo, Z.G., Wang, R.J. \& Luo, Y. 2016 Role of the inter-protofilament sliding in the bending of protein microtubules. Journal of Biomechanics 49, 3803-3807.

[83] Wang, N. \& Stamenovic, D. 2002 Mechanics of vimentin intermediate filaments. Journal of Muscle Research \& Cell Motility 23, 535-540.

[84] Wang, N., Butler, J.P. \& Ingber, D.E. 1993 Mechanotransduction across the cell surface and through the cytoskeleton. Science 260, 1124-1127.

[85] Fudge, D., Russell, D., Beriault, D., Moore, W., Lane, E.B. \& Vogl, A.W. 2008 The intermediate filament network in cultured human keratinocytes is remarkably extensible and resilient. PloS One 3, e2327.

[86] Huisman, E.M., van Dillen, T., Onck, P.R. \& Van der Giessen, E. 2007 Three-dimensional cross-linked F-actin networks: relation between network architecture and mechanical behavior. Physical Review Letters 99, 208103. 
[87] Gardel, M., Shin, J., MacKintosh, F., Mahadevan, L., Matsudaira, P. \& Weitz, D. 2004 Elastic behavior of cross-linked and bundled actin networks. Science 304, 1301-1305.

[88] Wilson, C.A., Tsuchida, M.A., Allen, G.M., Barnhart, E.L., Applegate, K.T., Yam, P.T., Ji, L., Keren, K., Danuser, G. \& Theriot, J.A. 2010 Myosin II contributes to cell-scale actin network treadmilling through network disassembly. Nature $\mathbf{4 6 5}$, 373.

[89] MacKintosh, F., Käs, J. \& Janmey, P. 1995 Elasticity of semiflexible biopolymer networks. Physical Review Letters 75, 4425.

[90] Kroy, K. \& Frey, E. 1996 Force-Extension Relation and Plateau Modulus for Wormlike Chains. Physical Review Letters 77, 306-309.

[91] Palmer, J.S. \& Boyce, M.C. 2008 Constitutive modeling of the stress-strain behavior of F-actin filament networks. Acta Biomaterialia 4, 597-612.

[92] Broedersz, C. \& MacKintosh, F. 2011 Molecular motors stiffen non-affine semiflexible polymer networks. Soft matter 7, 3186-3191.

[93] Unterberger, M.J., Schmoller, K.M., Bausch, A.R. \& Holzapfel, G.A. 2013 A new approach to model cross-linked actin networks: multi-scale continuum formulation and computational analysis. journal of the mechanical behavior of biomedical materials 22, 95-114.

[94] Wang, N. \& Ingber, D.E. 1995 Probing transmembrane mechanical coupling and cytomechanics using magnetic twisting cytometry. Biochemistry and Cell Biology 73, $327-335$.

[95] Nava, M.M., Raimondi, M.T. \& Pietrabissa, R. 2014 Bio-chemo-mechanical models for nuclear deformation in adherent eukaryotic cells. Biomechanics and modeling in mechanobiology 13, 929-943.

[96] Satcher, R.L. \& Dewey, C.F. 1996 Theoretical estimates of mechanical properties of the endothelial cell cytoskeleton. Biophysical Journal 71, 109-118.

[97] Gibson, L. \& Ashby, M. 1999 Cellular solids: structure and properties, 
Cambridge university press.

[98] Satcher, R., Dewey, C.F. \& Hartwig, J.H. 1997 Mechanical remodeling of the endothelial surface and actin cytoskeleton induced by fluid flow. Microcirculation $\mathbf{4}$, 439-453.

[99] Åström, J.A., Kumar, P.S., Vattulainen, I. \& Karttunen, M. 2008 Strain hardening, avalanches, and strain softening in dense cross-linked actin networks. Physical Review E 77, 051913.

[100] Åström, J.A., Kumar, P.S. \& Karttunen, M. 2009 Aster formation and rupture transition in semi-flexible fiber networks with mobile cross-linkers. Soft matter $\mathbf{5}$, 2869-2874.

[101] Huisman, E.M., Storm, C. \& Barkema, G.T. 2010 Frequency-dependent stiffening of semiflexible networks: A dynamical nonaffine to affine transition. Physical Review E 82, 061902.

[102] Huisman, E.M., Heussinger, C., Storm, C. \& Barkema, G.T. 2010 Semiflexible filamentous composites. Physical Review Letters 105, 118101.

[103] Huisman, E.M., Storm, C. \& Barkema, G.T. 2008 Monte Carlo study of multiply crosslinked semiflexible polymer networks. Physical Review E 78, 051801. [104] Huisman, E.M. \& Lubensky, T.C. 2011 Internal Stresses, Normal Modes, and Nonaffinity in Three-Dimensional Biopolymer Networks. Physical Review Letters 106, 088301.

[105] Dasanayake, N.L., Michalski, P.J. \& Carlsson, A.E. 2011 General mechanism of actomyosin contractility. Physical Review Letters 107, 118101.

[106] Peter, S.J. \& Mofrad, M.R. 2012 Computational modeling of axonal microtubule bundles under tension. Biophysical Journal 102, 749-757.

[107] Kang, J., Steward, R.L., Kim, Y., Schwartz, R.S., LeDuc, P.R. \& Puskar, K.M. 2011 Response of an actin filament network model under cyclic stretching through a coarse grained Monte Carlo approach. Journal of Theoretical Biology 274, 109-119. [108] Wang, S. \& Wolynes, P.G. 2012 Active contractility in actomyosin networks. 
Proceedings of the National Academy of Sciences 109, 6446-6451.

[109] Abhilash, A., Purohit, P.K. \& Joshi, S.P. 2012 Stochastic rate-dependent elasticity and failure of soft fibrous networks. Soft matter 8, 7004-7016.

[110] Carlsson, A.E. 2001 Growth of branched actin networks against obstacles. Biophysical Journal 81, 1907-1923.

[111] Loisel, T.P., Boujemaa, R., Pantaloni, D. \& Carlier, M.-F. 1999 Reconstitution of actin-based motility of Listeria and Shigella using pure proteins. Nature 401, 613. [112] van Oudenaarden, A. \& Theriot, J.A. 1999 Cooperative symmetry-breaking by actin polymerization in a model for cell motility. Nature Cell Biology 1, 493.

[113] Mogilner, A. \& Oster, G. 2003 Force generation by actin polymerization II: the elastic ratchet and tethered filaments. Biophysical Journal 84, 1591-1605.

[114] Carlsson, A. 2003 Growth velocities of branched actin networks. Biophysical Journal 84, 2907-2918.

[115] Alberts, J.B. \& Odell, G.M. 2004 In silico reconstitution of Listeria propulsion exhibits nano-saltation. PLoS Biology 2, e412.

[116] Zhu, J. \& Mogilner, A. 2012 Mesoscopic model of actin-based propulsion. PLoS Computational Biology 8, e1002764.

[117] Ingber, D.E. 2003 Tensegrity I. Cell structure and hierarchical systems biology. Journal of Cell Science 116, 1157-1173.

[118] Ingber, D.E. 2006 Cellular mechanotransduction: putting all the pieces together again. The FASEB journal 20, 811-827.

[119] Coughlin, M.F. \& Stamenović, D. 2003 A prestressed cable network model of the adherent cell cytoskeleton. Biophysical Journal 84, 1328-1336.

[120] Paul, R., Heil, P., Spatz, J.P. \& Schwarz, U.S. 2008 Propagation of mechanical stress through the actin cytoskeleton toward focal adhesions: model and experiment. Biophysical Journal 94, 1470-1482.

[121] Ingber, D. \& Jamieson, J. 1985 Cells as tensegrity structures: Architectural regulation of histodifferentiationby physical forces tranduced over basement 
membranes. Gene Expression during Normal and Malignent Differentiation.

[122] Boey, S.K., Boal, D.H. \& Discher, D.E. 1998 Simulations of the erythrocyte cytoskeleton at large deformation. I. Microscopic models. Biophysical Journal 75, 1573-1583.

[123] Ingber, D.E. 1993 Cellular tensegrity: defining new rules of biological design that govern the cytoskeleton. Journal of Cell Science 104, 613-627.

[124] Luo, Y., Xu, X., Lele, T., Kumar, S. \& Ingber, D.E. 2008 A multi-modular tensegrity model of an actin stress fiber. Journal of Biomechanics 41, 2379-2387.

[125] Orr, A.W., Helmke, B.P., Blackman, B.R. \& Schwartz, M.A. 2006 Mechanisms of mechanotransduction. Developmental Cell 10, 11-20.

[126] Shafrir, Y. \& Forgacs, G. 2002 Mechanotransduction through the cytoskeleton. American Journal of Physiology-Cell Physiology 282, C479-C486.

[127] Zeng, Y., Yip, A.K., Teo, S.-K. \& Chiam, K.-H. 2012 A three-dimensional random network model of the cytoskeleton and its role in mechanotransduction and nucleus deformation. Biomechanics and modeling in mechanobiology 11, 49-59.

[128] Head, D.A., Levine, A.J. \& MacKintosh, F.C. 2003 Deformation of Cross-Linked Semiflexible Polymer Networks. Physical Review Letters 91, 108102. [129] Head, D., Levine, A. \& MacKintosh, F. 2003 Distinct regimes of elastic response and deformation modes of cross-linked cytoskeletal and semiflexible polymer networks. Physical Review E 68, 061907.

[130] Wilhelm, J. \& Frey, E. 2003 Elasticity of Stiff Polymer Networks. Physical Review Letters 91, 108103.

[131] Onck, P., Koeman, T., Van Dillen, T. \& van der Giessen, E. 2005 Alternative explanation of stiffening in cross-linked semiflexible networks. Physical Review Letters 95, 178102.

[132] Roy, S. \& Qi, H.J. 2008 Micromechanical model for elasticity of the cell cytoskeleton. Physical Review E 77, 061916.

[133] Kaunas, R. \& Hsu, H.-J. 2009 A kinematic model of stretch-induced stress 
fiber turnover and reorientation. Journal of Theoretical Biology 257, 320-330.

[134] Kaunas, R. \& Deguchi, S. 2011 Multiple roles for myosin II in tensional homeostasis under mechanical loading. Cellular and molecular bioengineering $\mathbf{4}$, 182-191.

[135] Gong, J., Zhang, D., Tseng, Y., Li, B., Wirtz, D. \& Schafer, B.W. 2013 Form-finding model shows how cytoskeleton network stiffness is realized. PloS One 8, e77417.

[136] Parameswaran, H., Lutchen, K.R. \& Suki, B. 2014 A computational model of the response of adherent cells to stretch and changes in substrate stiffness. Journal of Applied Physiology 116, 825-834.

[137] Li, T., Hu, D., Yarlagadda, P.K. \& Gu, Y. 2014 Physical mechanism of the compressive response of F-actin networks: significance of crosslinker unbinding events. Theoretical and Applied Mechanics Letters 4, 051006-051001.

[138] Li, T., Gu, Y., Feng, X.-Q., Yarlagadda, P.K. \& Oloyede, A. 2013 Hierarchical multiscale model for biomechanics analysis of microfilament networks. Journal of Applied Physics 113, 194701.

[139] Klinge, S., Aygün, S., Gilbert, R. \& Holzapfel, G. 2018 Multiscale FEM simulations of cross-linked actin network embedded in cytosol with the focus on the filament orientation. International journal for numerical methods in biomedical engineering, e2993.

[140] Kroy, K. \& Glaser, J. 2007 The glassy wormlike chain. New Journal of Physics 9, 416.

[141] Wolff, L., Fernandez, P. \& Kroy, K. 2010 Inelastic mechanics of sticky biopolymer networks. New Journal of Physics 12, 053024.

[142] Broedersz, C.P., Depken, M., Yao, N.Y., Pollak, M.R., Weitz, D.A. \& MacKintosh, F.C. 2010 Cross-Link-Governed Dynamics of Biopolymer Networks. Physical Review Letters 105, 238101.

[143] Mofrad, M.R. 2009 Rheology of the cytoskeleton. Annual Review of Fluid 
Mechanics 41, 433-453.

[144] Kim, T., Hwang, W. \& Kamm, R. 2009 Computational analysis of a cross-linked actin-like network. Experimental Mechanics 49, 91-104.

[145] Kim, T., Hwang, W. \& Kamm, R.D. 2011 Dynamic role of cross-linking proteins in actin rheology. Biophysical Journal 101, 1597-1603.

[146] Borau, C., Kim, T., Bidone, T., García-Aznar, J.M. \& Kamm, R.D. 2012 Dynamic mechanisms of cell rigidity sensing: insights from a computational model of actomyosin networks. PloS One 7, e49174.

[147] Alvarado, J., Sheinman, M., Sharma, A., MacKintosh, F.C. \& Koenderink, G.H. 2013 Molecular motors robustly drive active gels to a critically connected state. Nature Physics 9, 591.

[148] Alonso, R., Young, J. \& Cheng, Y. 2014 A particle interaction model for the simulation of biological, cross-linked fiber networks inspired from flocking theory. Cellular and molecular bioengineering 7, 58-72.

[149] Wu, T. \& Feng, J.J. 2015 A biomechanical model for fluidization of cells under dynamic strain. Biophysical Journal 108, 43-52.

[150] Fallqvist, B. \& Kroon, M. 2016 Constitutive modelling of composite biopolymer networks. Journal of Theoretical Biology 395, 51-61.

[151] Guzik, B.W. \& Goldstein, L.S. 2004 Microtubule-dependent transport in neurons: steps towards an understanding of regulation, function and dysfunction. Current Opinion in Cell Biology 16, 443-450.

[152] Buxton, G.A., Siedlak, S.L., Perry, G. \& Smith, M.A. 2010 Mathematical modeling of microtubule dynamics: insights into physiology and disease. Progress in Neurobiology 92, 478-483.

[153] Allain, P. \& Kervrann, C. 2014 Physical modeling of microtubules network. In Journal of Physics: Conference Series (p. 012005, IOP Publishing.)

[154] Qin, Z., Buehler, M.J. \& Kreplak, L. 2010 A multi-scale approach to understand the mechanobiology of intermediate filaments. Journal of Biomechanics 
43, $15-22$.

[155] Ackbarow, T., Sen, D., Thaulow, C. \& Buehler, M.J. 2009 Alpha-helical protein networks are self-protective and flaw-tolerant. PloS One 4, e6015.

[156] Bertaud, J., Qin, Z. \& Buehler, M.J. 2010 Intermediate filament-deficient cells are mechanically softer at large deformation: a multi-scale simulation study. Acta Biomaterialia 6, 2457-2466.

[157] Baudriller, H., Maurin, B., Cañadas, P., Montcourrier, P., Parmeggiani, A. \& Bettache, N. 2006 Form-finding of complex tensegrity structures: application to cell cytoskeleton modelling. Comptes rendus mécanique 334, 662-668.

[158] Das, M., Levine, A.J. \& MacKintosh, F. 2008 Buckling and force propagation along intracellular microtubules. EPL (Europhysics Letters) 84, 18003.

[159] Valdman, D., Atzberger, P.J., Yu, D., Kuei, S. \& Valentine, M.T. 2012 Spectral analysis methods for the robust measurement of the flexural rigidity of biopolymers. Biophysical Journal 102, 1144-1153.

[160] Tounsi, A., Heireche, H., Benhassaini, H. \& Missouri, M. 2010 Vibration and length-dependent flexural rigidity of protein microtubules using higher order shear deformation theory. Journal of Theoretical Biology 266, 250-255.

[161] Takasone, T., Juodkazis, S., Kawagishi, Y., Yamaguchi, A., Matsuo, S., Sakakibara, H., Nakayama, H. \& Misawa, H. 2002 Flexural rigidity of a single microtubule. Japanese Journal of Applied Physics 41, 3015.

[162] Felgner, H., Frank, R. \& Schliwa, M. 1996 Flexural rigidity of microtubules measured with the use of optical tweezers. Journal of Cell Science 109, 509-516.

[163] Kikumoto, M., Kurachi, M., Tosa, V. \& Tashiro, H. 2006 Flexural rigidity of individual microtubules measured by a buckling force with optical traps. Biophysical Journal 90, 1687-1696.

[164] Howard, J. 2005 Mechanics of motor proteins and the cytoskeleton, Sinauer.

[165] Panyukov, S. \& Rabin, Y. 2000 Thermal fluctuations of elastic filaments with spontaneous curvature and torsion. Physical Review Letters 85, 2404. 
[166] Havelka, D., Deriu, M.A., Cifra, M. \& Kučera, O. 2017 Deformation pattern in vibrating microtubule: Structural mechanics study based on an atomistic approach. Scientific Reports 7, 4227.

[167] Zhang, J. \& Meguid, S. 2014 Buckling of microtubules: An insight by molecular and continuum mechanics. Applied Physics Letters 105, 173704.

[168] Hunyadi, V., Chretien, D., Flyvbjerg, H. \& Janosi, I.M. 2007 Why is the microtubule lattice helical? Biologie Cellulaire 99, 117-128.

[169] Hawkins, T., Mirigian, M., Selcuk Yasar, M. \& Ross, J.L. 2010 Mechanics of microtubules. Journal of Biomechanics 43, 23-30.

[170] Meurer-Grob, P., Kasparian, J. \& Wade, R.H. 2001 Microtubule structure at improved resolution. Biochemistry 40, 8000-8008.

[171] Weaver, W. \& Gere, J.M. 2012 Matrix Analysis of Framed Structures, Springer Science \& Business Media.

[172] Wade, R. 1997 Microtubule structure and dynamics. Current Opinion in Cell Biology 9, 12-17.

[173] Hunyadi, V., Chretien, D. \& Janosi, I.M. 2005 Mechanical stress induced mechanism of microtubule catastrophes. Journal of Molecular Biology 348, 927-938. [174] Li, C.Y. \& Chou, T.W. 2003 A structural mechanics approach for the analysis of carbon nanotubes. International Journal of Solids and Structures 40, 2487-2499.

[175] Enemark, S., Deriu, M.A., Soncini, M. \& Redaelli, A. 2008 Mechanical model of the tubulin dimer based on molecular dynamics simulations. Journal of Biomechanical Engineering 130, 041008.

[176] Wade, R.H. 2009 On and around microtubules: an overview. Molecular Biotechnology 43, 177-191.

[177] Dhatt, G., Touzot, G. \& Cantin, G. 1984 The Finite Element Method Displayed. John Wiley\&Sons, Chichester.

[178] de Pablo, P.J., Schaap, I.A., MacKintosh, F.C. \& Schmidt, C.F. 2003 Deformation and collapse of microtubules on the nanometer scale. Physical Review 
Letters 91, 098101.

[179] Sept, D. \& MacKintosh, F.C. 2010 Microtubule elasticity: connecting all-atom simulations with continuum mechanics. Physical Review Letters 104, 018101.

[180] Kawaguchi, K. \& Yamaguchi, A. 2010 Temperature dependence rigidity of non-taxol stabilized single microtubules. Biochemical and Biophysical Research Communications 402, 66-69.

[181] Wegner, A. 1976 Head to tail polymerization of actin. Journal of Molecular Biology 108, 139-150.

[182] Egelman, E., Francis, N. \& DeRosier, D. 1982 F-actin is a helix with a random variable twist. Nature 298, 131.

[183] Bourne, G.H. 2014 Structure and Function of Muscle, Elsevier.

[184] Egelman, E.H. 1985 The structure of F-actin. Journal of Muscle Research and Cell Motility 6, 129-151.

[185] Fritz, M., Radmacher, M., Cleveland, J.P., Allersma, M.W., Stewart, R.J., Gieselmann, R., Janmey, P., Schmidt, C.F. \& Hansma, P.K. 1995 Imaging globular and filamentous proteins in physiological buffer solutions with tapping mode atomic force microscopy. Langmuir 11, 3529-3535.

[186] Phillips, J.C., Braun, R., Wang, W., Gumbart, J., Tajkhorshid, E., Villa, E., Chipot, C., Skeel, R.D., Kale, L. \& Schulten, K. 2005 Scalable molecular dynamics with NAMD. Journal of Computational Chemistry 26, 1781-1802.

[187] Otterbein, L.R., Graceffa, P. \& Dominguez, R. 2001 The crystal structure of uncomplexed actin in the ADP state. Science 293, 708-711.

[188] Berman, H.M., Westbrook, J., Feng, Z., Gilliland, G., Bhat, T.N., Weissig, H., Shindyalov, I.N. \& Bourne, P.E. 2000 The protein data bank. Nucleic Acids Research 28, 235-242.

[189] Berendsen, H.J., Postma, J.v., van Gunsteren, W.F., DiNola, A. \& Haak, J. 1984 Molecular dynamics with coupling to an external bath. The Journal of chemical physics 81, 3684-3690. 
[190] Tang, M., Li, T., Gandhi, N.S., Burrage, K. \& Gu, Y. 2017 Heterogeneous nanomechanical properties of type I collagen in longitudinal direction. Biomechanics and modeling in mechanobiology 16, 1023-1033.

[191] Acun, B., Hardy, D., Kale, L.V., Li, K., Phillips, J. \& Stone, J. 2018 Scalable molecular dynamics with NAMD on the Summit system. IBM Journal of Research and Development 62, 4: 1-4: 9.

[192] MacKerell Jr, A.D., Bashford, D., Bellott, M., Dunbrack Jr, R.L., Evanseck, J.D., Field, M.J., Fischer, S., Gao, J., Guo, H. \& Ha, S. 1998 All-atom empirical potential for molecular modeling and dynamics studies of proteins. The Journal of Physical Chemistry B 102, 3586-3616.

[193] Phillips, J., Isgro, T., Sotomayor, M. \& Villa, E. 2007 Namd tutorial. (ed. N.R.f.M.M.a.B.B. Institute), University of Illinois.

[194] Wohlert, J. \& Edholm, O. 2004 The range and shielding of dipole-dipole interactions in phospholipid bilayers. Biophysical Journal 87, 2433-2445.

[195] Martens, C., Shekhar, M., Borysik, A.J., Lau, A.M., Reading, E., Tajkhorshid, E., Booth, P.J. \& Politis, A. 2018 Direct protein-lipid interactions shape the conformational landscape of secondary transporters. Nature communications $\mathbf{9}, 4151$. [196] Jorgensen, W.L., Chandrasekhar, J., Madura, J.D., Impey, R.W. \& Klein, M.L. 1983 Comparison of simple potential functions for simulating liquid water. The Journal of chemical physics 79, 926-935.

[197] Dupuis, D.E., Guilford, W.H., Wu, J. \& Warshaw, D.M. 1997 Actin filament mechanics in the laser trap. Journal of Muscle Research \& Cell Motility 18, 17-30. [198] Goldman, Y.E. \& Huxley, A.F. 1994 Actin compliance: are you pulling my chain? Biophysical Journal 67, 2131.

[199] Kojima, H., Ishijima, A. \& Yanagida, T. 1994 Direct measurement of stiffness of single actin filaments with and without tropomyosin by in vitro nanomanipulation. Proceedings of the National Academy of Sciences 91, 12962-12966.

[200] Prochniewicz, E., Janson, N., Thomas, D.D. \& De La Cruz, E.M. 2005 Cofilin 
Increases the Torsional Flexibility and Dynamics of Actin Filaments. Journal of Molecular Biology 353, 990-1000.

[201] Yoshimura, H., Nishio, T., Mihashi, K., Kinosita, K. \& Ikegami, A. 1984 Torsional motion of eosin-labeled F-actin as detected in the time-resolved anisotropy decay of the probe in the sub-millisecond time range. Journal of Molecular Biology 179, 453-467.

[202] Tsuda, Y., Yasutake, H., Ishijima, A. \& Yanagida, T. 1996 Torsional rigidity of single actin filaments and actin-actin bond breaking force under torsion measured directly by in vitro micromanipulation. Proceedings of the National Academy of Sciences 93, 12937-12942.

[203] Thomas, D.D., Seidel, J.C. \& Gergely, J. 1979 Rotational dynamics of spin-labeled F-actin in the sub-millisecond time range. Journal of Molecular Biology 132, 257-273.

[204] Prochniewicz, E., Zhang, Q., Janmey, P.A. \& Thomas, D.D. 1996 Cooperativity in F-actin: binding of gelsolin at the barbed end affects structure and dynamics of the whole filament. Journal of Molecular Biology 260, 756-766.

[205] Prochniewicz, E., Zhang, Q., Howard, E.C. \& Thomas, D.D. 1996 Microsecond rotational dynamics of actin: spectroscopic detection and theoretical simulation. Journal of Molecular Biology 255, 446-457.

[206] Prochniewicz, E., Walseth, T.F. \& Thomas, D.D. 2004 Structural dynamics of actin during active interaction with myosin: different effects of weakly and strongly bound myosin heads. Biochemistry 43, 10642-10652.

[207] Prochniewicz, E. \& Thomas, D.D. 1999 Differences in structural dynamics of muscle and yeast actin accompany differences in functional interactions with myosin. Biochemistry 38, 14860-14867.

[208] Prochniewicz, E. \& Thomas, D.D. 1997 Perturbations of functional interactions with myosin induce long-range allosteric and cooperative structural changes in actin. Biochemistry 36, 12845-12853. 
[209] Ostap, E.M., Yanagida, T. \& Thomas, D. 1992 Orientational distribution of spin-labeled actin oriented by flow. Biophysical Journal 63, 966-975.

[210] Moriyama, K. \& Yahara, I. 1999 Two activities of cofilin, severing and accelerating directional depolymerization of actin filaments, are affected differentially by mutations around the actin-binding helix. The EMBO Journal 18, $6752-6761$.

[211] Forkey, J.N., Quinlan, M.E. \& Goldman, Y.E. 2005 Measurement of single macromolecule orientation by total internal reflection fluorescence polarization microscopy. Biophysical Journal 89, 1261-1271.

[212] Egelman, E.H. \& DeRosier, D.J. 1992 Image analysis shows that variations in actin crossover spacings are random, not compensatory. Biophysical Journal 63, 1299-1305.

[213] Egelman, E. \& Padron, R. 1984 X-ray diffraction evidence that actin is a $100 \AA$ filament. Nature 307, 56-58.

[214] Oosawa, F. 1980 The flexibility of F-actin. Biophysical Chemistry 11, 443-446.

[215] Cherepanov, D.A. \& Junge, W. 2001 Viscoelastic Dynamics of Actin Filaments Coupled to Rotary F-ATPase: Curvature as an Indicator of the Torque. Biophysical Journal 81, 1234-1244.

[216] Pänke, O., Cherepanov, D.A., Gumbiowski, K., Engelbrecht, S. \& Junge, W. 2001 Viscoelastic Dynamics of Actin Filaments Coupled to Rotary F-ATPase: Angular Torque Profile of the Enzyme. Biophysical Journal 81, 1220-1233.

[217] Brangwynne, C.P., Koenderink, G.H., Barry, E., Dogic, Z., MacKintosh, F.C. \& Weitz, D.A. 2007 Bending dynamics of fluctuating biopolymers probed by automated high-resolution filament tracking. Biophysical Journal 93, 346-359.

[218] Gere, J.M. \& Goodno, B.J. 2012 Mechanics of materials, Nelson Education.

[219] Zhao, J., Wang, L., Jiang, J.-W., Wang, Z., Guo, W. \& Rabczuk, T. 2013 A comparative study of two molecular mechanics models based on harmonic potentials. Journal of Applied Physics 113, 063509. 
[220] Venier, P., Maggs, A.C., Carlier, M.-F. \& Pantaloni, D. 1994 Analysis of microtubule rigidity using hydrodynamic flow and thermal fluctuations. Journal of Biological Chemistry 269, 13353-13360.

[221] Kurachi, M., Hoshi, M. \& Tashiro, H. 1995 Buckling of a single microtubule by optical trapping forces: direct measurement of microtubule rigidity. Cell Motility and the Cytoskeleton 30, 221-228.

[222] Wang, N., Naruse, K., Stamenović, D., Fredberg, J.J., Mijailovich, S.M., Tolić-Nørrelykke, I.M., Polte, T., Mannix, R. \& Ingber, D.E. 2001 Mechanical behavior in living cells consistent with the tensegrity model. Proceedings of the National Academy of Sciences 98, 7765-7770.

[223] Vinckier, A., Dumortier, C., Engelborghs, Y. \& Hellemans, L. 1996 Dynamical and mechanical study of immobilized microtubules with atomic force microscopy. Journal of Vacuum Science \& Technology B: Microelectronics and Nanometer Structures Processing, Measurement, and Phenomena 14, 1427-1431.

[224] Gu, B., Mai, Y.-W. \& Ru, C.Q. 2009 Mechanics of microtubules modeled as orthotropic elastic shells with transverse shearing. Acta Mechanica 207, 195-209.

[225] Fu, Y.M. \& Zhang, J. 2010 Modeling and analysis of microtubules based on a modified couple stress theory. Physica E: Low-dimensional Systems and Nanostructures 42, 1741-1745.

[226] Tedesco, J.W., McDougal, W.G. \& Ross, C.A. 1999 Structural dynamics: theory and applications, Addison-Wesley Montlo Park, California.

[227] Li, C. \& Chou, T.-W. 2004 Vibrational behaviors of multiwalled-carbon-nanotube-based nanomechanical resonators. Applied Physics Letters 84, 121-123.

[228] Tuszyński, J., Luchko, T., Portet, S. \& Dixon, J. 2005 Anisotropic elastic properties of microtubules. The European Physical Journal E 17, 29-35.

[229] Reddy, J. \& Pang, S. 2008 Nonlocal continuum theories of beams for the analysis of carbon nanotubes. Journal of Applied Physics 103, 023511. 
[230] Arash, B. \& Wang, Q. 2012 A review on the application of nonlocal elastic models in modeling of carbon nanotubes and graphenes. Computational Materials Science 51, 303-313.

[231] Reddy, J. 2007 Nonlocal theories for bending, buckling and vibration of beams. International Journal of Engineering Science 45, 288-307.

[232] Eringen, A.C. 1983 On differential equations of nonlocal elasticity and solutions of screw dislocation and surface waves. Journal of Applied Physics 54, 4703-4710.

[233] Eringen, A.C. 1976 Nonlocal polar field theories. Continuum physics 4, 205-264.

[234] Civalek, Ö. \& Demir, Ç. 2011 Bending analysis of microtubules using nonlocal Euler-Bernoulli beam theory. Applied Mathematical Modelling 35, 2053-2067.

[235] Civalek, Ö. \& Akgöz, B. 2010 Free vibration analysis of microtubules as cytoskeleton components: nonlocal Euler-Bernoulli beam modeling. Scientia Iranica. Transaction B, Mechanical Engineering 17, 367.

[236] Dye, R.B., Fink, S.P. \& Williams, R.C. 1993 Taxol-induced flexibility of microtubules and its reversal by MAP-2 and Tau. Journal of Biological Chemistry 268, 6847-6850.

[237] Janson, M.E. \& Dogterom, M. 2004 A bending mode analysis for growing microtubules: evidence for a velocity-dependent rigidity. Biophysical Journal 87, 2723-2736.

[238] Howard, J. \& Clark, R. 2002 Mechanics of motor proteins and the cytoskeleton. Applied Mechanics Reviews 55, B39.

[239] Allen, K.B., Sasoglu, F.M. \& Layton, B.E. 2009 Cytoskeleton-membrane interactions in neuronal growth cones: a finite analysis study. Journal of Biomechanical Engineering 131, 021006.

[240] Molodtsov, M.I., Ermakova, E.A., Shnol, E.E., Grishchuk, E.L., McIntosh, J.R. \& Ataullakhanov, F.I. 2005 A molecular-mechanical model of the microtubule. 
Biophysical Journal 88, 3167-3179.

[241] Pokorný, J., Vedruccio, C., Cifra, M. \& Kučera, O. 2011 Cancer physics: diagnostics based on damped cellular elastoelectrical vibrations in microtubules. European Biophysics Journal 40, 747-759.

[242] Iijima, S. 1991 Helical microtubules of graphitic carbon. Nature 354, 56-58.

[243] Jishi, R.A., Dresselhaus, M.S. \& Dresselhaus, G. 1993 Electron-phonon coupling and the electrical conductivity of fullerene nanotubules. Physical review B 48, 11385 .

[244] Pampaloni, F. \& Florin, E.L. 2008 Microtubule architecture: inspiration for novel carbon nanotube-based biomimetic materials. Trends in Biotechnology 26, 302-310.

[245] Lim, Y.C., Cooling, M.T. \& Long, D.S. 2015 Computational models of the primary cilium and endothelial mechanotransmission. Biomechanics and modeling in mechanobiology 14, 665-678.

[246] Barreto, S., Clausen, C.H., Perrault, C.M., Fletcher, D.A. \& Lacroix, D. 2013 A multi-structural single cell model of force-induced interactions of cytoskeletal components. Biomaterials 34, 6119-6126.

[247] Jin, M.Z. \& Ru, C.Q. 2013 Localized buckling of a microtubule surrounded by randomly distributed cross linkers. Physical Review E 88, 012701.

[248] Oosawa, F., Fujime, S., Ishiwata, S.i. \& Mihashi, K. 1973 Dynamic property of F-actin and thin filament. In Cold Spring Harbor Symposia on Quantitative Biology (pp. 277-285, Cold Spring Harbor Laboratory Press.

[249] Brangwynne, C.P., MacKintosh, F.C., Kumar, S., Geisse, N.A., Talbot, J., Mahadevan, L., Parker, K.K., Ingber, D.E. \& Weitz, D.A. 2006 Microtubules can bear enhanced compressive loads in living cells because of lateral reinforcement. Journal of Cell Biology 173, 733-741.

[250] Kawaguchi, K., Ishiwata, S.i. \& Yamashita, T. 2008 Temperature dependence of the flexural rigidity of single microtubules. Biochemical and Biophysical Research 
Communications 366, 637-642.

[251] Gittes, F., Meyhöfer, E., Baek, S. \& Howard, J. 1996 Directional loading of the kinesin motor molecule as it buckles a microtubule. Biophysical Journal 70, 418-429.

[252] Yi, L., Chang, T. \& Ru, C. 2008 Buckling of microtubules under bending and torsion. Journal of Applied Physics 103, 103516.

[253] Shen, H.S. 2010 Nonlocal shear deformable shell model for postbuckling of axially compressed microtubules embedded in an elastic medium. Biomechanics and modeling in mechanobiology 9, 345-357.

[254] Jiang, H. \& Zhang, J. 2008 Mechanics of microtubule buckling supported by cytoplasm. Journal of Applied Mechanics 75, 061019.

[255] Hirokawa, N. 1982 Cross-linker system between neurofilaments, microtubules and membranous organelles in frog axons revealed by the quick-freeze, deep-etching method. The Journal of cell biology 94, 129.

[256] Rodriguez, O.C., Schaefer, A.W., Mandato, C.A., Forscher, P., Bement, W.M. \& Waterman-Storer, C.M. 2003 Conserved microtubule-actin interactions in cell movement and morphogenesis. Nature Cell Biology 5, 599-609.

[257] Mattila, P.K. \& Lappalainen, P. 2008 Filopodia: molecular architecture and cellular functions. Nature Reviews Molecular Cell Biology 9, 446-454.

[258] Footer, M.J., Kerssemakers, J.W., Theriot, J.A. \& Dogterom, M. 2007 Direct measurement of force generation by actin filament polymerization using an optical trap. Proceedings of the National Academy of Sciences 104, 2181-2186.

[259] Li, C. \& Chou, T.-W. 2004 Modeling of elastic buckling of carbon nanotubes by molecular structural mechanics approach. Mechanics of Materials 36, 1047-1055. [260] Hyman, A.A., Chrétien, D., Arnal, I. \& Wade, R.H. 1995 Structural changes accompanying GTP hydrolysis in microtubules: information from a slowly hydrolyzable analogue guanylyl-(alpha, beta)-methylene-diphosphonate. The Journal of cell biology 128, 117-125. 
[261] Bathe, M., Heussinger, C., Claessens, M.M., Bausch, A.R. \& Frey, E. 2008 Cytoskeletal bundle mechanics. Biophysical Journal 94, 2955-2964.

[262] Claessens, M.M., Bathe, M., Frey, E. \& Bausch, A.R. 2006 Actin-binding proteins sensitively mediate F-actin bundle stiffness. Nature materials $\mathbf{5}$, 748-753.

[263] Sanger, J.W., Sanger, J.M. \& Jockusch, B.M. 1983 Differences in the stress fibers between fibroblasts and epithelial cells. The Journal of cell biology 96, 961-969.

[264] Bartles, J.R. 2000 Parallel actin bundles and their multiple actin-bundling proteins. Current Opinion in Cell Biology 12, 72-78.

[265] Adams, J.C. 2004 Roles of fascin in cell adhesion and motility. Current Opinion in Cell Biology 16, 590-596.

[266] Tilney, M.S., Tilney, L.G., Stephens, R.E., Merte, C., Drenckhahn, D., Cotanche, D.A. \& Bretscher, A. 1989 Preliminary biochemical characterization of the stereocilia and cuticular plate of hair cells of the chick cochlea. The Journal of cell biology 109, 1711-1723.

[267] Lin, C.-S., Shen, W., Chen, Z.P., Tu, Y.-H. \& Matsudaira, P. 1994 Identification of I-plastin, a human fimbrin isoform expressed in intestine and kidney. Molecular and Cellular Biology 14, 2457-2467.

[268] Ishikawa, R., Sakamoto, T., Ando, T., Higashi - Fujime, S. \& Kohama, K. 2003 Polarized actin bundles formed by human fascin - 1: their sliding and disassembly on myosin II and myosin V in vitro. Journal of Neurochemistry 87, 676-685.

[269] Claessens, M.M.A.E., Semmrich, C., Ramos, L. \& Bausch, A. 2008 Helical twist controls the thickness of F-actin bundles. Proceedings of the National Academy of Sciences 105, 8819-8822.

[270] Mogilner, A. \& Rubinstein, B. 2005 The physics of filopodial protrusion. Biophysical Journal 89, 782-795.

[271] Vignjevic, D., Kojima, S.-i., Aratyn, Y., Danciu, O., Svitkina, T. \& Borisy, G.G. 
2006 Role of fascin in filopodial protrusion. Journal of Cell Biology 174, 863-875.

[272] Cohan, C.S., Welnhofer, E.A., Zhao, L., Matsumura, F. \& Yamashiro, S. 2001

Role of the actin bundling protein fascin in growth cone morphogenesis: localization in filopodia and lamellipodia. Cytoskeleton 48, 109-120.

[273] Winder, S.J. \& Ayscough, K.R. 2005 Actin-binding proteins. Journal of Cell Science 118, 651-654.

[274] Stracke, R., Böhm, K., Wollweber, L., Tuszynski, J. \& Unger, E. 2002 Analysis of the migration behaviour of single microtubules in electric fields. Biochemical and Biophysical Research Communications 293, 602-609.

[275] Nogales, E., Wolf, S.G. \& Downing, K.H. 1998 Structure of the $\alpha \beta$ tubulin dimer by electron crystallography. Nature 391, 199-203.

[276] Pizzi, R., Strini, G., Fiorentini, S., Pappalardo, V. \& Pregnolato, M. 2011 Evidences of new biophysical properties of microtubules. In Artificial Neural Networks Engineering tools, techniques and tables Mathematics research developments series (ed. S.J. Kwon), Nova Science Publishers.

[277] Cifra, M., Pokorný, J., Havelka, D. \& Kučera, O. 2010 Electric field generated by axial longitudinal vibration modes of microtubule. BioSystems 100, 122-131.

[278] Pokorný, J. 2003 Viscous effects on polar vibrations in microtubules. Electromagnetic Biology and Medicine 22, 15-29.

[279] Kasas, S., Cibert, C., Kis, A., Rios, P.L., Riederer, B., Forro, L., Dietler, G. \& Catsicas, S. 2004 Oscillation modes of microtubules. Biology of the Cell 96, 697-700.

[280] Arani, A.G., Abdollahian, M. \& Jalaei, M. 2015 Vibration of bioliquid-filled microtubules embedded in cytoplasm including surface effects using modified couple stress theory. Journal of Theoretical Biology 367, 29-38.

[281] Barzanjeh, S., Salari, V., Tuszynski, J., Cifra, M. \& Simon, C. 2017 Optomechanical proposal for monitoring microtubule mechanical vibrations. Physical Review E 96, 012404. 
[282] Foster, K.R. \& Baish, J.W. 2000 Viscous damping of vibrations in microtubules. Journal of Biological Physics 26, 255-260.

[283] Kučera, O., Havelka, D. \& Cifra, M. 2017 Vibrations of microtubules: Physics that has not met biology yet. Wave Motion 72, 13-22.

[284] Longhurst, M. \& Quirke, N. 2006 The environmental effect on the radial breathing mode of carbon nanotubes in water. The Journal of chemical physics $\mathbf{1 2 4}$, 234708 .

[285] Rao, A., Chen, J., Richter, E., Schlecht, U., Eklund, P., Haddon, R., Venkateswaran, U., Kwon, Y.-K. \& Tomanek, D. 2001 Effect of van der Waals interactions on the Raman modes in single walled carbon nanotubes. Physical Review Letters 86, 3895.

[286] Hameroff, S. \& Penrose, R. 2014 Consciousness in the universe: A review of the 'Orch OR'theory. Physics of life reviews 11, 39-78.

[287] Vater, W., Stracke, R., Boehm, K., Speicher, C., Weber, P. \& Unger, E. 1998 Behaviour of individual microtubules and microtubule bundles in electric fields. In Sixth Foresight Conference on Molecular Nanotechnology (Westin Hotel in Santa Clara, California, US, Foresight Institute and the Institute for Molecular Manufacturing.)

[288] Vassilev, P.M., Dronzine, R.T., Vassileva, M.P. \& Georgiev, G.A. 1982 Parallel arrays of microtubles formed in electric and magnetic fields. Bioscience Reports 2 , 1025-1029.

[289] Vassilev, P. \& Kanazirska, M. 1985 The role of cytoskeleton in the mechanisms of electric field effects and information transfer in cellular systems. Medical Hypotheses 16, 93-96.

[290] Brown, J.A.M. 2000 A Study of the Interactions between Electromagnetic Fields and Microtubules: Ferroelectric Effects, Signal Transduction and Electronic Conduction. Edmonton/Alberta/Canada, University of Alberta.

[291] Mershin, A., Kolomenski, A.A., Schuessler, H.A. \& Nanopoulos, D.V. 2004 
Tubulin dipole moment, dielectric constant and quantum behavior: computer simulations, experimental results and suggestions. BioSystems 77, 73-85.

[292] Grant, I.S. \& Phillips, W.R. 2013 Electromagnetism. Chichester, John Wiley \& Sons.

[293] Zahn, M. 1979 Electromagnetic Field Theory: a problem solving approach. New York, John Wiley \& Sons.

[294] Griffiths, D.J. 1999 Introduction to electrodynamics. New Jersey, Prentice Hall.

[295] WHO. Electromagnetic fields (EMF). (available at http://www.who.int/peh-emf/about/WhatisEMF/en/index3.html).

[296] Chang, H., Zhang, Y., Xie, J., Zhou, Z. \& Yuan, W. 2010 Integrated behavior simulation and verification for a MEMS vibratory gyroscope using parametric model order reduction. Journal of Microelectromechanical Systems 19, 282-293.

[297] Cho, Y.H., Kwak, B.M., Pisano, A.P. \& Howe, R.T. 1994 Slide film damping in laterally driven microstructures. Sensors and Actuators A: Physical 40, 31-39.

[298] Liu, X., Civet, Y. \& Perriard, Y. 2015 Quality factor and vibration amplitude estimation of a piezoelectric-actuated system using impedance measurements. In 2015 18th International Conference on Electrical Machines and Systems (ICEMS) (pp. 1993-1996. Pattaya, Thailand, IEEE.

[299] Piersol, A.G. \& Harris, C.M. 2017 Harri's Shock and Vibration Handbook Fifth Edition, Mcgraw-hill.

[300] Krivosudský, O. \& Cifra, M. 2016 Microwave absorption by nanoresonator vibrations tuned with surface modification. EPL (Europhysics Letters) 115, 44003.

[301] Newmark, N.M. 1959 A method of computation for structural dynamics. Journal of the engineering mechanics division 85, 67-94.

[302] Landau, L. \& Lifshitz, E. 1987 Fluid Mechanics 2nd Edition England, Pergamon Press.

[303] Cho, Y.H., Kwak, B.M., Pisano, A.P. \& Howe, R.T. 1993 Viscous energy 
dissipation in laterally oscillating planar microstructures: A theoretical and experimental study. In [1993] Proceedings IEEE Micro Electro Mechanical Systems (pp. 93-98. Fort Lauderdale, FL, USA, IEEE.

[304] Zhang, X. 2002 Frequency analysis of submerged cylindrical shells with the wave propagation approach. International Journal of Mechanical Sciences 44, 1259-1273.

[305] Wang, C.Y., Zhang, J., Fei, Y.Q. \& Murmu, T. 2012 Circumferential nonlocal effect on vibrating nanotubules. International Journal of Mechanical Sciences 58, $86-90$.

[306] Wang, C., Ru, C. \& Mioduchowski, A. 2005 Free vibration of multiwall carbon nanotubes. Journal of Applied Physics 97, 114323.

[307] Luo, Q., Kuang, D., Zhang, B. \& Song, G. 2016 Cell stiffness determined by atomic force microscopy and its correlation with cell motility. Biochimica et Biophysica Acta (BBA)-General Subjects 1860, 1953-1960.

[308] Löwe, J., Li, H., Downing, K. \& Nogales, E. 2001 Refined structure of $\alpha \beta$-tubulin at 3.5 Å resolution. Journal of Molecular Biology 313, 1045-1057.

[309] Tischfield, M.A., Cederquist, G.Y., Gupta, M.L. \& Engle, E.C. 2011 Phenotypic spectrum of the tubulin-related disorders and functional implications of disease-causing mutations. Current Opinion in Genetics \& Development 21, 286-294.

[310] Tu, K., Tobias, D.J. \& Klein, M.L. 1995 Constant pressure and temperature molecular dynamics simulation of a fully hydrated liquid crystal phase dipalmitoylphosphatidylcholine bilayer. Biophysical Journal 69, 2558-2562.

[311] Darden, T., York, D. \& Pedersen, L. 1993 Particle mesh Ewald: An N· log (N) method for Ewald sums in large systems. The Journal of chemical physics 98, 10089-10092.

[312] Li, S., Wang, C. \& Nithiarasu, P. 2019 Electromechanical vibration of microtubules and its application in biosensors. Journal of the Royal Society Interface 
16, 20180826. 